\title{
Concentrations of Selected Herbicides, Herbicide Degradation Products, and Nutrients in the Lower Mississippi River, Louisiana, April 1991 through December 2003
}

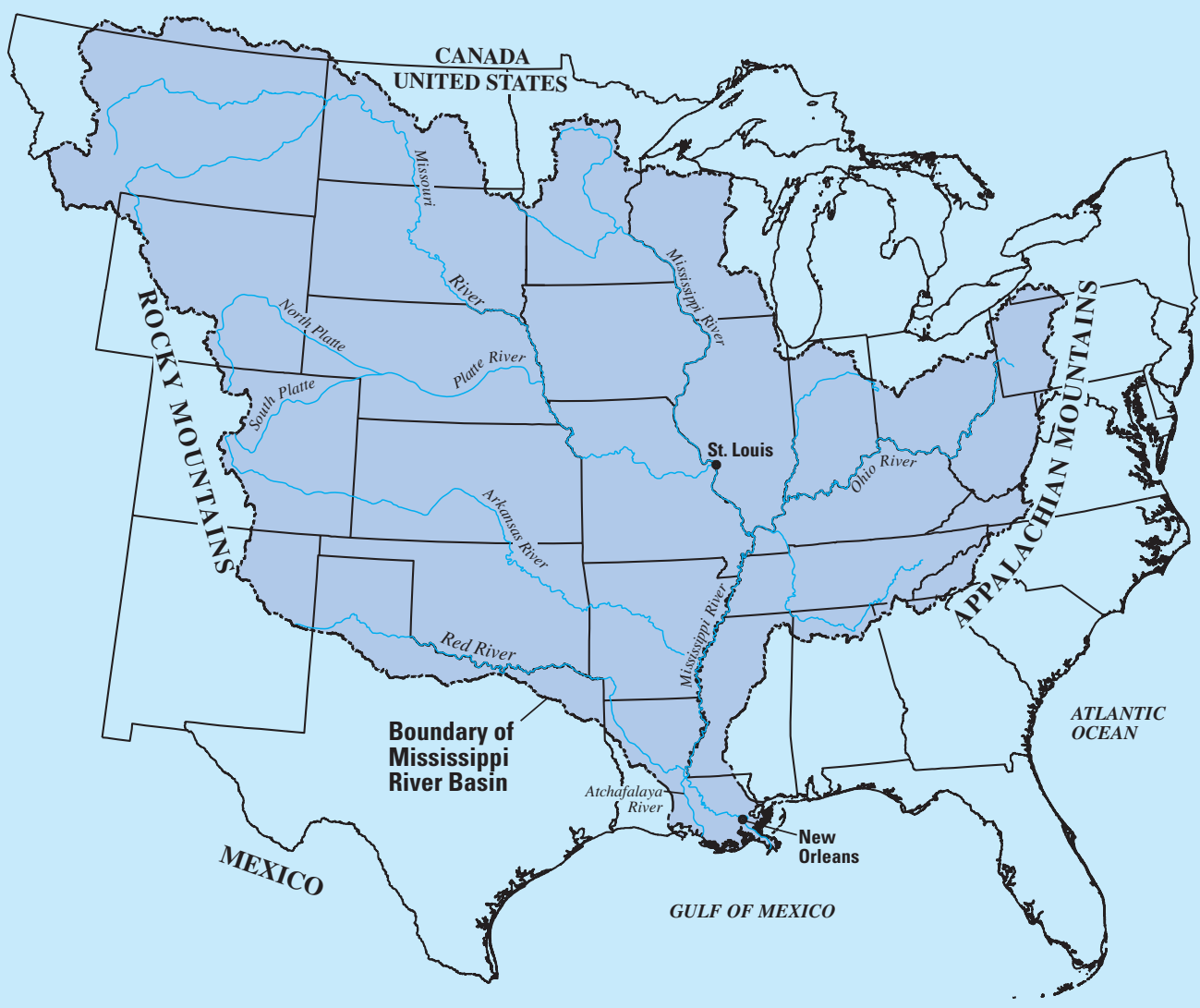

Data Series 165 


\section{Concentrations of Selected Herbicides, Herbicide Degradation Products, and Nutrients in the Lower Mississippi River, Louisiana, April 1991 through December 2003}

By Elisabeth A. Scribner, Donald A. Goolsby, William A. Battaglin, Michael T. Meyer, and E.M. Thurman

Data Series 165 


\title{
U.S. Department of the Interior Gale A. Norton, Secretary
}

\author{
U.S. Geological Survey \\ P. Patrick Leahy, Acting Director
}

\section{U.S. Geological Survey, Reston, Virginia: 2006}

For sale by U.S. Geological Survey, Information Services

Box 25286, Denver Federal Center

Denver, CO 80225

For more information about the USGS and its products:

Telephone: 1-888-ASK-USGS

World Wide Web: http://www.usgs.gov/

Any use of trade, product, or firm names in this publication is for descriptive purposes only and does not imply endorsement by the U.S. Government.

Although this report is in the public domain, permission must be secured from the individual copyright owners to reproduce any copyrighted materials contained within this report.

Suggested citation:

Scribner, E.A., Goolsby, D.A., Battaglin, W.A., Meyer, M.T., and Thurman, E.M., 2006, Concentrations of selected herbicides, herbicide degradation products, and nutrients in the lower Mississippi River, Louisiana, April 1991 through December 2003: U.S. Geological Survey Data Series 165, 84 p. 


\section{Contents}

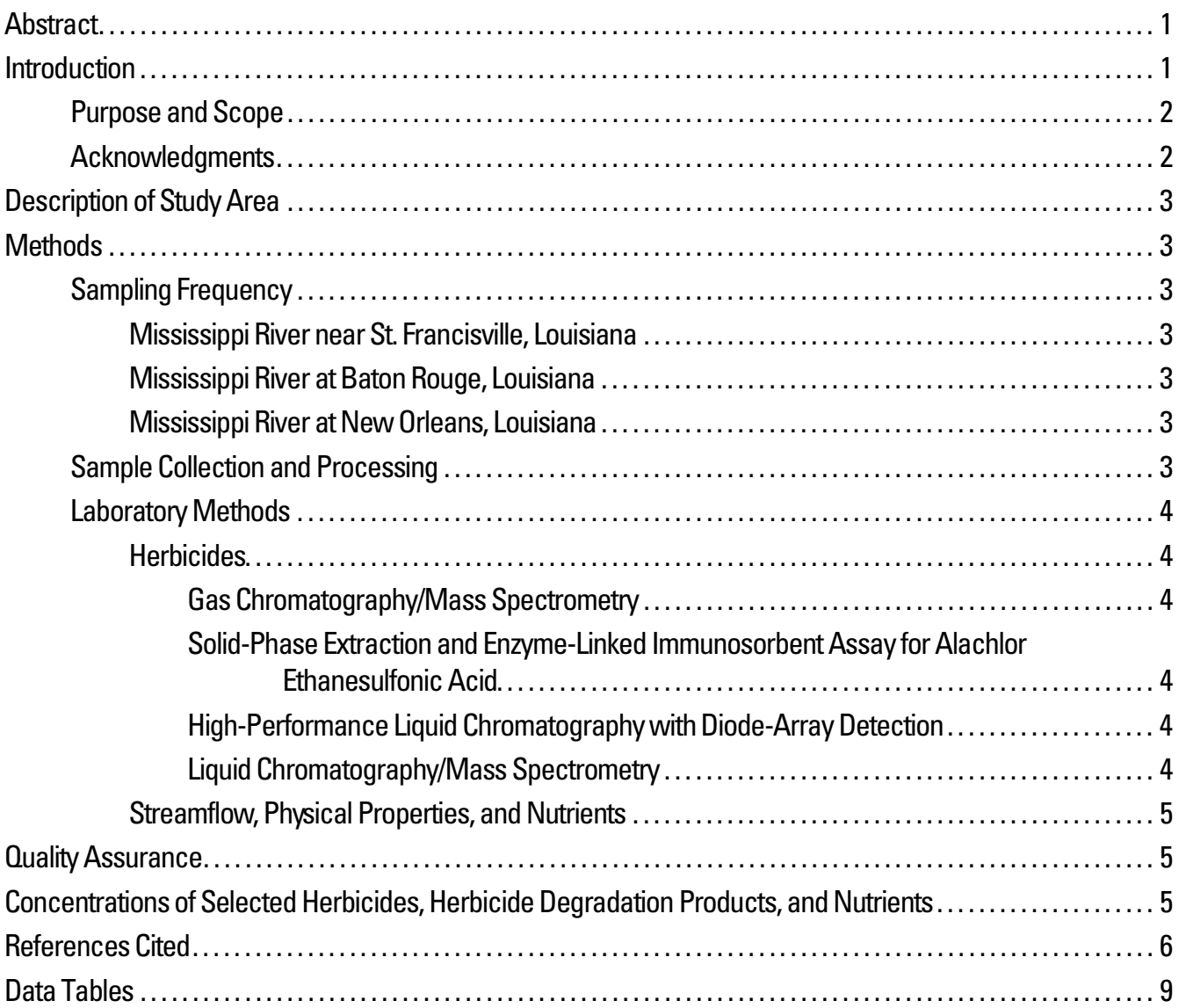

\section{Figure}

1. Map showing location of Mississippi River Basin, major tributaries, and St. Francisville, Baton Rouge, and New Orleans, Louisiana, sampling sites ......................................

\section{Tables}

1. Herbicides and their degradation products, years sampled, and method of analysis for water samples collected from the lower Mississippi River in Louisiana, 1991-2003. ...

2. Results of analysis of selected herbicides and degradation products by the U.S. Geological Survey National Water-Quality Laboratory, Lakewood, Colorado, for water samples collected from the Mississippi River near St. Francisville and at Baton Rouge, Louisiana, 1991-2003.

3. Results of analysis of alachlor, atrazine, metolachlor, and simazine by the Jefferson Parish Water Quality Laboratory, Jefferson, Louisiana, for water samples collected from the Mississippi River at New Orleans, Louisiana, March through September 1993 ............................. 23

4. Results of analysis of selected triazine herbicides and degradation products by gas chromatography/ mass spectrometry methods GCS and GCR at the U.S. Geological Survey Organic Geochemistry 
Research Laboratory, Lawrence, Kansas, for water samples collected from the Mississippi River at

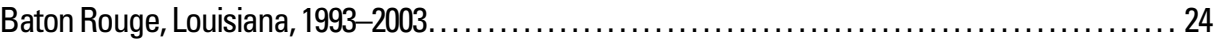

5. Results of analysis of selected acetamide herbicides and degradation products by liquid chromatography/mass spectrometry methods HPAA, LCAA, and LCPD atthe U.S. Geological Survey Organic Geochemistry Research Laboratory, Lawrence, Kansas, for water samples collected from the Mississippi River at Baton Rouge, Louisiana, 1998-2003.

6. Results of analysis of selected triazine and phenylurea herbicides and their degradation products by liquid chromatography/mass spectrometry method LCEA at the U.S. Geological Survey Organic Geochemistry Research Laboratory, Lawrence, Kansas, for water samples collected from the Mississippi River at Baton Rouge, Louisiana, 1998-2003. 54

7. Results of analysis of glyphosate, its degradation product aminomethylphosphonic acid, and glufosinate by liquid chromatography/mass spectrometry method LCGY at the U.S. Geological Survey Organic Geochemistry Research Laboratory, Lawrence, Kansas, for water samples collected from the Mississippi River at Baton Rouge, Louisiana, 2001-03. . .

8. Results of analysis of streamflow, physical properties, and nutrients for water samples collected from three sampling sites in the lower Mississippi River, Louisiana, 1991-2003.

9. Statistical summary of concentrations of selected herbicides and degradation products analyzed by the U.S. Geological Survey National Water-Quality Laboratory, Lakewood, Colorado, for water samples collected from the Mississippi River near St. Francisville and at Baton Rouge, Louisiana, 19912003

10. Statistical summary of concentrations of selected herbicides analyzed by the Jefferson Parish Water Quality Laboratory, Jefferson, Louisiana, for water samples collected from the Mississippi River at New Orleans, Louisiana, March through September 1993.

11. Statistical summary of concentrations of selected triazine herbicides and degradation products analyzed by gas chromatography/mass spectrometry methods GCS and GCR at the U.S. Geological Survey Organic Geochemistry Research Laboratory, Lawrence, Kansas, for water samples collected from the Mississippi River at Baton Rouge, Louisiana, 1993-2003. 79

12. Statistical summary of concentrations of selected acetamide herbicides and degradation products analyzed by liquid chromatography/mass spectrometry methods HPAA, LCAA, and LCPD at the U.S. Geological Survey Organic Geochemistry Research Laboratory, Lawrence, Kansas, for water samples collected from the Mississippi River at Baton Rouge, Louisiana, 1998-2003. ... 80

13. Statistical summary of concentrations of selected triazine and phenylurea herbicides and degradation products analyzed by liquid chromatography/mass spectrometry method LCEA at the U.S. Geological Survey Organic Geochemistry Research Laboratory, Lawrence, Kansas, for water samples collected from the Mississippi River at Baton Rouge, Louisiana, 1998-2003.

14. Statistical summary of concentrations of glyphosate, its degradation product aminomethylphosphonic acid, and glufosinate analyzed by liquid chromatography/mass spectrometry method LCGY at the U.S. Geological Survey Organic Geochemistry Research Laboratory, Lawrence, Kansas, for water samples collected from the Mississippi River at Baton Rouge, Louisiana, 2001-03. ...

15. Statistical summary of streamflow, physical properties, and nutrients analyzed for water samples collected from three sampling sites in the lower Mississippi River, Louisiana, 1991-2003. .... 83 


\section{Conversion Factors, Abbreviated Water-Quality Units, and Datum}

\begin{tabular}{lll}
\hline Multiply & By & To obtain \\
\hline cubic foot per second $\left(\mathrm{ft}^{3} / \mathrm{s}\right)$ & 0.02832 & cubic meter per second $\left(\mathrm{m}^{3} / \mathrm{s}\right)$ \\
foot $(\mathrm{ft})$ & 0.3048 & meter $(\mathrm{m})$ \\
inch per year $(\mathrm{in} / \mathrm{yr})$ & 2.54 & centimeter per year $(\mathrm{cm} / \mathrm{yr})$ \\
microliter $(\mu \mathrm{L})$ & 0.00003382 & ounce $(\mathrm{oz})$ \\
micrometer $(\mu \mathrm{m})$ & 0.00003937 & inch $(\mathrm{in})$. \\
mile $(\mathrm{mi})$ & 1.609 & kilometer $(\mathrm{km})$ \\
milliliter $(\mathrm{mL})$ & 0.0338 & ounce, fluid $(\mathrm{oz})$ \\
square mile $\left(\mathrm{mi}^{2}\right)$ & 2.590 & square kilometer $\left(\mathrm{km}^{2}\right)$ \\
\hline
\end{tabular}

Temperature in degrees Celsius $\left({ }^{\circ} \mathrm{C}\right)$ may be converted to degrees Fahrenheit $\left({ }^{\circ} \mathrm{F}\right)$ as follows:

${ }^{\circ} \mathrm{F}=\left(1.8 \mathrm{x}^{\circ} \mathrm{C}\right)+32$.

Temperature in degrees Fahrenheit $\left({ }^{\circ} \mathrm{F}\right)$ may be converted to degrees Celsius $\left({ }^{\circ} \mathrm{C}\right)$ as follows:

${ }^{\circ} \mathrm{C}=\left({ }^{\circ} \mathrm{F}-32\right) / 1.8$.

\section{Abbreviated Water-Quality Units}

microgram per liter $(\mu \mathrm{g} / \mathrm{L})$

microsiemens per centimeter at 25 degrees Celsius $(\mu \mathrm{S} / \mathrm{cm})$

milligram per liter (mg/L)

\section{Datum}

Horizontal coordinate information is referenced to the North American Datum of 1983 (NAD 83). 


\title{
Concentrations of Selected Herbicides, Herbicide Degradation Products, and Nutrients in the Lower Mississippi River, Louisiana, April 1991 through December 2003
}

\author{
By Elisabeth A. Scribner, Donald A. Goolsby, William A. Battaglin, Michael T. Meyer, and E.M. Thurman
}

\section{Abstract}

Water-quality samples were collected from three sites in the lower Mississippi River in Louisiana during April 1991 through December 2003 by the U.S. Geological Survey and analyzed for selected herbicides, herbicide degradation products, and nutrients (nitrogen, phosphorus, and silica). When this study first began in April 1991, water samples were analyzed for selected herbicides and herbicide degradation products by gas chromatography/mass spectrometry. As the study progressed, there were changes and additions to the herbicide compounds analyzed, which resulted in lower analytical reporting levels or the quantification of additional herbicides or herbicide degradation products. Alachlor ethanesulfonic acid was analyzed by solid-phase extraction and enzyme-linked immunosorbent assay from 1993 through 1998. In March 1998, analyses for the acetamide degradation products, ethanesulfonic acid and oxanilic acid, by high-performance liquid chromatography with diode-array detection, were added. This method was replaced by liquid chromatography/mass spectrometry in August 1999.

The data were collected to help determine the occurrence and transport of selected herbicides, their degradation products, and nutrients in the lower Mississippi River Basin and their delivery to the Gulf of Mexico. In addition to results of sample analysis, this report includes a description of the study area, sample-collection schedule and methods, laboratory analytical methods, and quality-assurance procedures.

\section{Introduction}

This is the ninth in a series of U.S. Geological Survey (USGS) water-quality reports that present the analytical results from studies of herbicide and nutrient occurrence in water resources of the Midwestern United States. This report presents the analytical results from long-term monitoring of herbicides, herbicide degradation products, and nutrients in the lower Mississippi River in Louisiana. Previous reports have presented analytical results from regional studies of herbicides and nutrients in ground water (Kolpin and others, 1993), surface water (Scribner and others, 1993), storm runoff (Scribner and others, 1994), precipitation (Goolsby and others, 1995), and reservoirs (Scribner and others, 1996), results from a study of the effects of changes in herbicide application rates (Scribner and others, 1998), results from a study of glyphosate occurrence (Scribner and others, 2003; Battaglin and others, 2005), and a summary of study results for triazine herbicides and their degradation products in water from the Midwestern United States during the 1990s (Scribner and others, 2005).

Previous studies have shown that runoff from watersheds within the Mississippi River Basin often contains significant amounts of herbicides and nutrients, such as nitrate (Goolsy and others, 1991a, 1991b, 1995; Thurman and others, 1992; Battaglin and others, 1993; Goolsby and Battaglin, 1993, 2000, 2001; Meade, 1995; Coupe and others, 1995; Clark and Goolsby, 1999; Clark and others, 1999; Coupe and Goolsby, 1999; Scribner and others, 2000). A portion of these herbicides and nutrients are transported into the Mississippi River and eventually into the Gulf of Mexico. The concentrations and mass transport (loads) of these compounds have been monitored by the USGS at two sites on the lower Mississippi River, St. Francisville and Baton Rouge (fig. 1). Long-term monitoring has been conducted by the USGS National Stream Quality Accounting Network (NASQAN) on the Mississippi River at St. Francisville, Louisiana, since 1955. Recently, more intensive monitoring has been conducted at Baton Rouge, Louisiana, about 85 mi downstream from St. Francisville (Coupe and Goolsby, 1999). In addition, the Jefferson Parish Water Treatment Plant monitors for selected herbicides and nitrate in the Mississippi River at New Orleans (fig. 1; Goolsby and others, 1993; Clark and others, 1999). The analytical results from these three sites provide a comprehensive record of the concentrations of herbicide compounds and nitrate in the lower Mississippi River in Louisiana for April 1991 through 2003. 


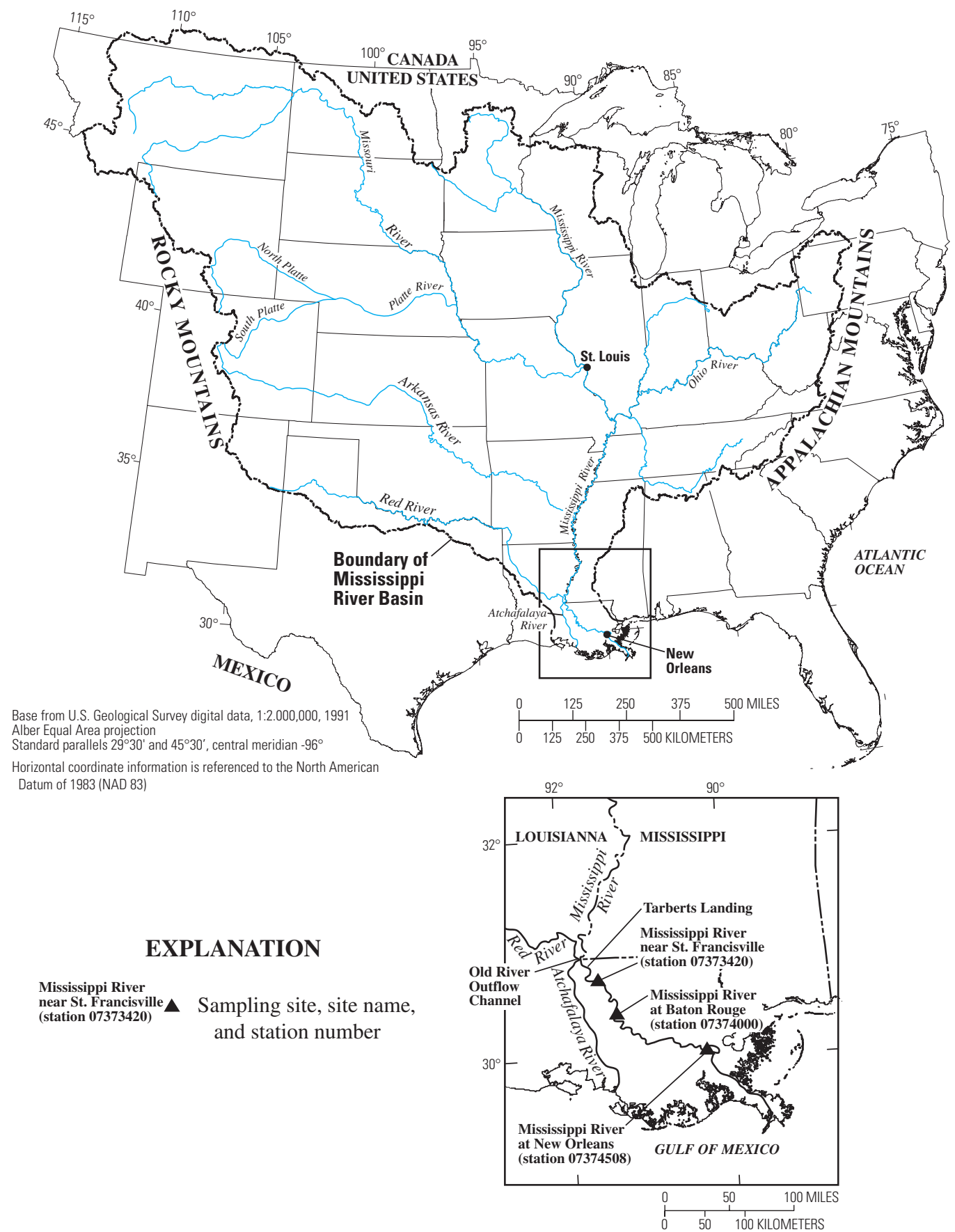

Figure 1. Location of Mississippi River Basin, major tributaries, and St. Francisville, Baton Rouge, and New Orleans, Louisiana, sampling sites.

\section{Purpose and Scope}

The purpose of this report is to present the results of laboratory analysis of water-quality samples collected to determine concentrations of selected herbicides, herbicide degradation products, and nutrients in the lower Mississippi River at St. Francisville, Baton Rouge, and New Orleans, Louisiana, for the period April 1991 through December 2003. This report also describes the study area, the methods used to collect the sam- ples, laboratory analytical methods, and quality-assurance procedures.

\section{Acknowledgments}

The authors wish to thank USGS employees in the Louisiana Water Science Center (in particular, Charles Demas and Dennis Demcheck) in Baton Rouge for collecting water sam- 
ples from the Mississippi River at St. Francisville and Baton Rouge. The authors thank Mr. Wayne Koffskey of the Jefferson Parish Water Quality Laboratory for providing data from the New Orleans sampling site. Also, thanks to Mr. John Miller of the U.S. Army Corps of Engineers for providing streamflow data for the lower Mississippi River. This study would not have been possible without their assistance.

\section{Description of Study Area}

The Mississippi River and its tributary, the Atchafalaya River, drain an area of more than $1,238,800 \mathrm{mi}^{2}$ or about 41 percent of the conterminous United States (fig. 1). The Mississippi River Basin is the largest river basin in North America. Its drainage covers much of the Central United States, including parts or all of 30 States extending from the Appalachian Mountains to the Rocky Mountains and from southern Canada to the Gulf of Mexico. About 72 million people live in the basin. The climate, land use, soils, and population density vary widely across the basin. The annual runoff ranges from less than $2 \mathrm{in} / \mathrm{yr}$ in the arid western part of the basin to more than $24 \mathrm{in} / \mathrm{yr}$ in the humid eastern part. About 58 percent of the basin is in cropland, whereas other land uses include woodland, range and barren land, wetlands and water, and urban land (Goolsby and Battaglin, 2000).

The majority of all agricultural chemicals used in the United States are applied to cropland in the Mississippi River Basin (Goolsby and Battaglin, 2000). As a result of rainfall runoff and ground-water discharge, many agricultural chemicals used in the Midwestern States are transported to the Mississippi River and its tributaries, making water quality an important issue (Goolsby and others, 1991; Battaglin and others, 1993; Goolsby and Battaglin, 1993; Clark and Goolsby, 1999; Coupe and Goolsby, 1999; Goolsby and Battaglin, 2000; Coupe and others, 2005).

\section{METHODS}

\section{Sampling Frequency}

\section{Mississippi River near St. Francisville, Louisiana}

Samples were collected bimonthly by the USGS from April 1991 through September 1995 at the Mississippi River near St. Francisville, Louisiana, sampling site. Since October 1995, samples were collected monthly with additional samples collected during high flow (Coupe and Goolsby, 1999). The sampling schedule was as follows:

\begin{abstract}
May 1991-September 1995 6-12 samples per year;
October 1995-December 20031 sample per month plus additional samples during high-flow periods.
\end{abstract}

\title{
Mississippi River at Baton Rouge, Louisiana
}

Water samples were collected from April 1991 through December 2003 by the USGS at Baton Rouge, Louisiana (Goolsby and others, 1991b; Coupe and others, 1995). The sampling schedule was as follows:

April 1991-September 1992

October 1992-June 1993

July 1993-December 2003

\section{1-2 samples per week;} not sampled; approximately weekly during periods of high flow; 1-2 samples per month at other times.

\section{Mississippi River at New Orleans, Louisiana}

From October 1992 through June 1993, samples from the Mississippi River at New Orleans were collected and analyzed by the Jefferson Parish Water Quality Laboratory (Goolsby and others, 1993). This site is about 124 mi downstream from Baton Rouge. Nutrient samples from the Jefferson Parish Laboratory were used to complete the period from October 1992 through June 1993. Herbicide data from the Jefferson Parish Laboratory were used to complete the period from March 1993 through June 1993 when samples were not collected at Baton Rouge.

\section{Sample Collection and Processing}

Water samples from the St. Francisville and Baton Rouge sites were collected by USGS personnel. Samples from the St. Francisville site were collected by equal-discharge increment sampling procedures (Edwards and Glysson, 1988) using either a P-63 sampler or a Teflon bag sampler. Samples from three to five locations across the river were composited into a large glass container. Subsamples for analysis of herbicide compounds and nutrients were taken from the container. Samples from the Baton Rouge site were collected with a weighted bottle sampler from the upper $20 \mathrm{ft}$ of the water column at the end of a pier that extends about $148 \mathrm{ft}$ from shore. Previous work had indicated that the dissolved solutes in the river at this site are well-mixed vertically and horizontally (Coupe and others, 1995). Water samples from both sites were filtered through a $0.45-\mu \mathrm{m}$ membrane filter for nutrient analysis, and samples for herbicide analyses were filtered through a $0.7-\mu \mathrm{m}$ glass-fiber filter.

Water samples were collected at the New Orleans site by personnel of the Jefferson Parish Water Quality Laboratory. These samples were weekly composites collected continuously at two sampling points on opposite banks of the Mississippi River by using peristaltic pumps and storing the samples 


\section{Concentrations of Selected Herbicides, Herbicide Degradation Products, and Nutrients, Lower Mississippi River}

at $4{ }^{\circ} \mathrm{C}$. Samples from both locations were analyzed, and results were averaged.

\section{Laboratory Methods}

\section{Herbicides}

Laboratory methods and procedures for the analysis of herbicides changed throughout the course of this study. The type of herbicide, years sampled, and laboratory method analysis code for chemical compounds analyzed using gas chromatography/mass spectrometry (GC/MS), enzyme-linked immunosorbent assay (ELISA), high-performance liquid

chromatography diode-array detection (HPLC/DAD), and liquid chromatography/mass spectrometry (LC/MS) are shown in table 1. All tables are presented at the back of this report.

\section{Gas Chromatography/Mass Spectrometry}

From May 1991 to December 2003, samples were collected from the Mississippi River near St. Francisville, Louisiana, and from April 1991 to September 1992, samples were collected from the Mississippi River at Baton Rouge, Louisiana (table 2). These samples were analyzed for herbicides by GC/MS at the USGS National Water-Quality Laboratory (NWQL), Lakewood, Colorado. During 1991-94 NWQL Method 1379 was used (Sandstrom and others, 1992). After 1994, samples were analyzed using NWQL Method 2001 (Zaugg and others, 1995). Samples collected by Jefferson Parish Water Quality Laboratory at New Orleans, Louisiana, during March 1993 through September 1993 (table 3) were analyzed for herbicides by gas chromatography using U.S. Environmental Protection Agency protocols (Goolsby and others, 1993). Various analytical detection levels for NWQL Methods 1379 and 2001 and for the Jefferson Parish Water Quality Laboratory were reported. Beginning in July 1993, samples were extracted using a solid-phase extraction (SPE) procedure at the USGS Organic Geochemistry Research Laboratory (OGRL), Lawrence, Kansas (Thurman and others, 1990; Meyer and others, 1993). Samples were analyzed by two USGS methods, including method number 0-2132-99 (Zimmerman and Thurman, 1999), using the OGRL method analysis code of GCS from July 1993 to July 1996, and method number 0-2132-99 (Kish and others, 2000), using the OGRL method analysis code GCR from August 1996 to May 2003 (table 4). The analytical reporting limit for both GC/MS methods is $0.05 \mu \mathrm{g} / \mathrm{L}$.

\section{Solid-Phase Extraction and Enzyme-Linked Immunosorbent Assay for Alachlor Ethanesulfonic Acid}

Alachlor ethanesulfonic acid (ESA) was analyzed by SPE and ELISA (OGRL method analysis code IME) at the OGRL, Lawrence, Kansas (table 4). Results were quantified with solutions of alachlor concentration that ranged from 0 to $20 \mu \mathrm{g} / \mathrm{L}$.
Using the calibration curves, optical densities associated with calibration standards were examined. Samples were analyzed in duplicate and averaged. The reporting limit for alachlor ESA is $0.10 \mu \mathrm{g} / \mathrm{L}$ (Aga and others, 1994). The SPE-ELISA method was discontinued in September 1998 when a method that included alachlor ESA was developed using high-performance liquid chromatography (HPLC) with diode-array detection (DAD). Additional studies were published by Aga and others (1994) and Aga and Thurman (2001).

\section{High-Performance Liquid Chromatography with Diode-Array Detection}

From March 1998 through August 1999, water samples from the Mississippi River at Baton Rouge, Louisiana (table 5) were analyzed by HPLC/DAD for acetochlor, alachlor, and metolachlor degradation products of ESA and oxanilic acid (OXA). Recovery for HPLC/DAD is discussed in the work of Ferrer and others (1997). HPLC/DAD was suitable for the determination of low micrograms-per-liter concentrations of acetamide degradation products in natural water samples. The limit of quantitation for HPLC/DAD is $0.20 \mu \mathrm{g} / \mathrm{L}$. The approved method number is 0-2133-00 (Zimmerman and others, 2000), and the method is identified by OGRL analysis code HPAA.

\section{Liquid Chromatography/Mass Spectrometry}

Beginning in April 1999, herbicide samples were analyzed by both the LC/MS and the HPLC/DAD method for comparison. The HPLC/DAD method was discontinued in August 1999. Water samples were extracted and analyzed by four different LC/MS methods at the OGRL as follows:

1. Method number 0-2134-00, which was approved by USGS in April 2000 (Lee and others, 2001), was used to analyze 10 acetamide degradation products. This method was used on water samples from the Mississippi River at Baton Rouge, Louisiana, from 1999 through 2002 (table 5) and is identified by the OGRL analysis code LCAA. The analytical reporting limit for LCAA is $0.05 \mu \mathrm{g} / \mathrm{L}$.

2. Method number 0-2139-03, which was approved by USGS in May 2003 (Lee and Strahan, 2003), is an analytical method for the determination of six acetamide herbicides and 16 of their degradation products. This method was used on water samples from the Mississippi River at Baton Rouge, Louisiana, from December 2002 through December 2003 (table 5) and is identified by the OGRL analysis code LCPD. The analytical reporting limit for LCPD is $0.02 \mu \mathrm{g} / \mathrm{L}$.

3. Method number 0-2138-02 method, which was approved by USGS in December 2002 (Lee and others, 2002b), was used to analyze four triazine parent compounds and 11 triazine degradation products plus three phenylurea parent compounds and one phenylurea degradation product in samples from the Mississippi River at Baton 
Rouge, Louisiana, from August 1998 through December 2003 (Lee and others, 2002b; table 6) and is identified by the OGRL method analysis code LCEA. The method reporting levels for LCEA were $0.025 \mu \mathrm{g} / \mathrm{L}$ for all triazine herbicides except deethylcyanazine, which was $0.20 \mu \mathrm{g} / \mathrm{L}$. The method reporting level for phenylurea herbicides was $0.20 \mu \mathrm{g} / \mathrm{L}$.

4. Method number 0-2136-01, which was approved by USGS in December 2001 (Lee and others, 2002a), was used to analyze glyphosate, its degradation product aminomethylphosphonic acid (AMPA), and glufosinate in samples from the Mississippi River at Baton Rouge, Louisiana, from October 2001 through October 2003 (table 7) and is identified by the OGRL method analysis code LCGY (Lee and others, 2002a). The analytical reporting limit for $\mathrm{LCGY}$ is $0.10 \mu \mathrm{g} / \mathrm{L}$.

\section{Streamflow, Physical Properties, and Nutrients}

Streamflow data for the Mississippi River in Louisiana were obtained from the U.S. Army Corps of Engineers (USCOE), New Orleans District Web site (http://www.mvn.usace.army.mil/eng/edhd/Wcontrol/ discharge.htm). In northern Louisiana, about 25 percent of the flow of the Mississippi River is diverted into the Atchafalaya River via the Old River Outflow Channel (fig. 1). The flow diverted into the Old River Outflow Channel is reported by USCOE on their Web site. The remaining water flows down the Mississippi River through Baton Rouge and New Orleans to the Gulf of Mexico. This flow is measured at Tarberts Landing (fig. 1) and is also reported on the USCOE Web site. The total flow of the Mississippi River is the sum of the flows measured at the above two locations. The total streamflow, specific conductance, and water temperature associated with each sample for the days on which samples were collected are presented in table 8 at the back of this report.

Dissolved nitrite plus nitrate, nitrite, ammonia, orthophosphate, and silica (table 8) were determined by an automated colorimetric procedure. Dissolved nitrate was calculated as the difference between determinations for nitrite plus nitrate and nitrite (Fishman and Friedman, 1989).

\section{Quality Assurance}

Quality-assurance procedures for sample collection required that all bottles and sampling equipment be cleaned by washing glass containers, filter units, and tubing with a phosphate-free laboratory detergent; rinsing with tap water; organicfree, deionized, or distilled water; methanol; and rinsed again with organic-free water to remove traces of methanol. Herbicide sample containers (125-mL amber glass bottles) were cleaned by heating overnight to about $350^{\circ} \mathrm{C}$ (Goolsby and others, 1991b). Approximately 10 percent of all herbicide samples were analyzed in duplicate at OGRL, and results are presented in tables at the back of this report.

\section{Concentrations of Selected Herbicides, Herbicide Degradation Products, and Nutrients}

The results of sample analyses are given in tables $2-15$ at the back of this report. Results are reported by NWQL (Lakewood, Colorado), Jefferson Parish Water Quality Laboratory (Jefferson, Louisiana), and OGRL (Lawrence, Kansas). The results from OGRL are listed by method analysis codes, as described previously, and are accompanied by a statistical summary of the reported concentrations. For calculation of the statistics in these tables, all nondetections were treated as zeros. Data from this study are available in electronic form from USGS, OGRL (E-mail ks_orgl@usgs.gov).

The results for herbicides and degradation products analyzed by NWQL are listed in table 2. Statistical summaries of these results are given in table 9 at the back of this report. Atrazine was the most frequently detected compound, and deethylatrazine was the most frequently detected atrazine degradation product during 1991-2003. Cyanazine followed with 119 detections in 142 samples collected. The number of detections of acetochlor and metribuzin is notable with 105 detections in 118 samples collected and 110 detections in 114 samples collected, respectively.

The Jefferson Parish Water Quality Laboratory provided results for four herbicides, alachlor, atrazine, metolachlor, and simazine, March through September 1993 (table 3 at the back of this report). The statistical summary (table 10 at the back of this report) shows these four herbicides were found in most of the 26 samples collected with atrazine at a maximum concentration of $4.2 \mu \mathrm{g} / \mathrm{L}$. These data provide information for the lower Mississippi River during the time when samples were not being collected by USGS. In the summer of 1993, a persistent wetweather pattern resulted in flooding in the upper Mississippi River Basin. As a result of the flood, USGS began sample collection at several locations on the Mississippi River and in adjacent shallow wells after the floodwaters receded (Goolsby and others, 1993).

In July 1993, OGRL began analysis by GC/MS of 17 herbicides and eight herbicide degradation products for water samples collected from the Mississippi River at Baton Rouge, Louisiana, as shown in table 4 at the back of this report. Results of alachlor ESA by ELISA are also shown in table 4. The statistical summary (table 11 at the back of this report) shows that three herbicides, atrazine, cyanazine, and metolachlor, were detected most often of the 17 herbicides analyzed (table 4). Six of the herbicide degradation products, alachlor ESA, deethylatrazine, deisopropylatrazine, cyanazine amide, demethylfluometuron, and 3-trifluoromethylphenylurea, were detected out of the nine degradation products analyzed. Sample detection 


\section{Concentrations of Selected Herbicides, Herbicide Degradation Products, and Nutrients, Lower Mississippi River}

frequencies included 197 of 277 samples for deethylatrazine, 162 of 166 samples for alachlor ESA, 122 of 277 samples for deisopropylatrazine, and 65 of 215 samples for cyanazine amide. Demethylfluometuron and 3-trifluromethlphenylurea were detected in one sample each. These results compare well with results from NWQL (table 9) and agree with previous investigations (Goolsby and others, 1991a, 1991b; Thurman and others, 1991, 1992; Scribner and others, 2003, 2005).

Methods for analyzing acetamide herbicides and acetamide degradation products were upgraded as new instrumentation was purchased for OGRL. As a result, these herbicide compounds were analyzed by three different liquid chromatography methods (table 5). The statistical results are shown in table 12. Degradation products of metolachlor, metolachlor ESA and metolachlor OXA, and the degradation product of alachlor, alachlor ESA, were detected most frequently from 1997 through 2003. The highest concentration was metolachlor ESA at $1.3 \mu \mathrm{g} / \mathrm{L}$, and alachlor ESA had a maximum concentration of $0.44 \mu \mathrm{g} / \mathrm{L}$.

Two LC/MS methods more recently developed by OGRL include analysis of triazine and phenylurea herbicides plus their degradation products (table 6) using method analysis code LCEA and analysis of glyphosate, its degradation product AMPA, and glufosinate (table 7) using method analysis code LCGY. Thirty-two samples were analyzed by LCEA as shown by the summary in table 13 . Atrazine was detected in all 32 samples, and its degradation product deethylatrazine was detected in 30 of 32 samples. The maximum concentration was for atrazine at $1.3 \mu \mathrm{g} / \mathrm{L}$, and the minimum concentration was for deisopropylhydroxyatrazine, a degradation product of atrazine, at $0.04 \mu \mathrm{g} / \mathrm{L}$. There were three detections of diuron, which is a phenylurea herbicide.

The statistical summary for glyphosate, AMPA, and glufosinate analyzed using OGRL method analysis code LCGY is shown in table 14 . There were 35 samples analyzed with no detections of either glyphosate or glufosinate. However, there were 32 detections of the degradation product of glyphosate, AMPA, in the 35 samples collected, with a maximum concentration of $0.38 \mu \mathrm{g} / \mathrm{L}$. Results of analysis of water samples by the LCGY method can be found in Battaglin and others (2005).

Streamflow, physical property, and nutrient data are shown in table 8 for all three Mississippi River sampling sites described in this report. Statistical summaries of these data are given in table 15 at the back of this report. Details of nutrient sources, concentrations, trends, and mass transport (loads) are presented in Goolsby and others (1999) and Goolsby and Battaglin (2000, 2001).

\section{References Cited}

Aga, D.S., and Thurman, E.M., 2001, Formation and transport of the sulfonic acid metabolites of alachlor and metolachlor in soil: Environmental Science \& Technology, v. 34, p. 2455-2460.
Aga, D.S., Thurman, E.M., and Pomes, M.L., 1994, Determination of alachlor and its sulfonic acid degradation product in water by solid-phase extraction and enzyme-linked immunosorbent assay: Analytical Chemistry, v. 66, p. 1495-1499.

Battaglin, W.A., Goolsby, D.A., and Coupe, R.H., 1993, Annual use and transport of agricultural chemicals in the Mississippi River, 1991-92, in Goolsby, D.A., and others, eds., Selected papers on agricultural chemicals in water resources of the Midcontinental United States: U.S. Geological Survey Open-File Report 93-418, p. 26-38.

Battaglin, W.A., Kolpin, D.W., Scribner, E.A., Kuivila, K.M., and Sandstrom, M.W., 2005, Glyphosate, other herbicides, and transformation products in Midwestern streams, 2002: American Water Works Association, v. 41, no. 2, p. 323-332. Clark, G.M., and Goolsby, D.A., 1999, Discharge of herbicides from the Mississippi River Basin to the Gulf of Mexico, 1991-97: U.S. Geological Survey Fact Sheet 163-98, 4 p.

Clark, G.M., Goolsby, D.A., and Battaglin, W.A., 1999, Seasonal and annual load of herbicides from the Mississippi River Basin to the Gulf of Mexico: Environmental Science \& Technology, v. 33, no. 7, p. 981-986.

Coupe, R.H., and Goolsby, D.A., 1999, Monitoring the water quality of the Nation's large rivers, Mississippi River Basin NASQAN Program: U.S. Geological Survey Fact Sheet 05599, 6 p.

Coupe, R.H., Goolsby, D.A., Iverson, J.L., Markovchick, D.J., and Zaugg, S.D., 1995, Pesticide, nutrient, water-discharge, and physical-property data for the Mississippi River and some of its tributaries, April 1991-September 1992: U.S. Geological Survey Open-File Report 93-657, 116 p.

Coupe, R.H., Welch, H.L., Pell, A.B., and Thurman, E.M., 2005, Herbicide and degradate flux in the Yazoo River Basin: International Journal of Environmental Analytical Chemistry, v. 85 , no. 15 , p. 1127-1140.

Edwards, T.K., and Glysson, G.D., 1988, Field methods for measurement of fluvial sediment: U.S. Geological Survey Open-File Report 86-531, 118 p.

Ferrer, I., Thurman, E.M., and Barcelo, D., 1997, Identification of ionic chloroacetanilide-herbicide degradation products in surface water and ground water by HPLC/MS using negative ion spray: Analytical Chemistry, v. 69, no. 22, p. 45474553.

Fishman, M.J., and Friedman, L.C., 1989, Methods for the determination of inorganic substances in water and fluvial sediments: U.S. Geological Survey Techniques of WaterResources Investigations, book 5, chap. A1, 545 p.

Goolsby, D.A., and Battaglin, W.A., 1993, Occurrence, distribution, and transport of agricultural chemicals in surface waters of the Midwestern United States, in Goolsby, D.A., Boyer, L.L., and Mallard, G.E., eds., Selected papers on agricultural chemicals in water resources of the Midcontinental United States: U.S. Geological Survey Open-File Report 93418, p. 1-24. 
Goolsby, D.A., and Battaglin, W.A., 2000, Nitrogen in the Mississippi Basin-estimating sources and predicting flux to the Gulf of Mexico: U.S. Geological Survey Fact Sheet 135-00, $6 \mathrm{p}$.

Goolsby, D.A., and Battaglin, W.A., 2001, Long-term changes in concentrations and flux of nitrogen in the Mississippi River Basin, USA: Hydrological Processes, v. 15, p. 12091226.

Goolsby, D.A., Battaglin, W.A., Lawrence, G.B., Artz, R.S., Aulenbach, B.T., Hooper,R.P., Keeney, D.R., and Stensland, G.J., 1999, Flux and sources of nutrients in the MississippiAtchafalaya River Basin topic 3 report for the integrated assessment on hypoxia in the Gulf of Mexico: Silver Spring, Maryland, National Oceanic and Atmospheric Administration Coastal Ocean Office, NOAA Coastal Ocean Program Decision Analysis Series No. 17, 130 p.

Goolsby, D.A., Battaglin, W.A., and Thurman, E.M., 1993, Occurrence and transport of agricultural chemicals in the Mississippi River Basin, July through August, 1993: U.S. Geological Survey Circular 1120-C, 22 p.

Goolsby, D.A., Coupe, R.H., and Markovchick, D.J., 1991a, Distribution of selected herbicides and nitrate in the Mississippi River and its major tributaries, April through June 1991: U.S. Geological Survey Water-Resources Investigations Report 91-4163, 35 p.

Goolsby, D.A., Scribner, E.A., Thurman, E.M., Pomes, M.L., and Meyer, M.T., 1995, Data on selected herbicides and two triazine degradation products in precipitation in the Midwestern and Northeastern United States, 1990-91: U.S. Geological Survey Open-File Report 95-469, 341 p.

Goolsby, D.A., Thurman, E.M., and Kolpin, D.W., 1991b, Geographic and temporal distribution of herbicides in surface waters of the upper Midwestern United States, 1989-90, in Mallard, G.E., and Aronson, D.A., eds., U.S. Geological Survey Toxic Substances Hydrology Program- Proceedings of the technical meeting, Monterey, California, March 1115, 1991: U.S. Geological Survey Water-Resources Investigations Report 91-4034, p. 183-188.

Kish, J.L., Thurman, E.M., Scribner, E.A., and Zimmerman, L.R., 2000, Methods of analysis by the U.S. Geological Survey Organic Geochemistry Research Group_-determination of selected herbicides and their degradation products in water using solid-phase extraction and gas chromatography/mass spectrometry: U.S. Geological Survey Open-File Report 00-385, 13 p.

Kolpin, D.W., Burkart, M.R., and Thurman, E.M., 1993, Hydrogeologic, water-quality and land-use data for the reconnaissance of herbicides and nitrate in near-surface aquifers of the midcontinental United States: U.S. Geological Survey Open-File Report 93-114, 61 p.

Lee, E.A., Kish, J.L., Zimmerman, L.R., and Thurman, E.M., 2001, Methods of analysis by the U.S. Geological Survey Organic Geochemistry Research Group - update and additions to the determination of chloroacetanilide herbicide degradation compounds in water using high-performance liquid chromatography/mass spectrometry: U.S. Geological Survey Open-File Report 01-10, 17 p.

Lee, E.A., and Strahan, A.P., 2003, Methods of analysis by the U.S. Geological Survey Organic Geochemistry Research Group-determination of acetamide herbicides and their degradation products in water using online solid-phase extraction and high-performance liquid chromatography/mass spectrometry: U.S. Geological Survey Open-File Report 03-173, 17 p.

Lee, E.A., Strahan, A.P., and Thurman, E.M., 2002a, Methods of analysis by the U.S. Geological Survey Organic Geochemistry Research Group-determination of glyphosate, aminomethylphosphonic acid, and glufosinate in water using online solid-phase extraction and high-performance liquid chromatography/mass spectrometry: U.S. Geological Survey Open-File Report 01-454, 13 p.

Lee, E.A., Strahan, A.P., and Thurman, E.M., 2002b, Methods of analysis by the U.S. Geological Survey Organic Geochemistry Research Group-determination of triazine and phenylurea herbicides and their degradation products in water using solid-phase extraction and liquid chromatography/ mass spectrometry: U.S. Geological Survey Open-File Report 02-436, 19 p.

Meade, R.H., 1995, Contaminants in the Mississippi River: U.S. Geological Survey Circular 1133, 140 p.

Meyer, M.T., Mills, M.S., and Thurman, E.M., 1993, Automated solid-phase extraction on herbicides from water for gas chromatography/mass spectrometry analysis: Journal of Chromatography, v. 629, p. 55-59.

Sandstrom, M.W., Wydoski, D.S., Schroeder, M.P., Zamboni, J.L., and Foreman, W.T., 1992, Methods of analysis by the National Water Quality Laboratory-determination of organonitrogen herbicides in water by solid phase extraction and capillary column gas chromatograph/mass spectrometry with selected ion monitoring: U.S. Geological Survey Open-File Report 91-519, 26 p.

Scribner, E.A., Battaglin, W.A., Dietze, J.E., and Thurman, E.M., 2003, Reconnaissance data for glyphosate, other selected herbicides, their degradation products, and antibiotics in 51 streams in nine Midwestern States, 2002: U.S. Geological Survey Open-File Report 03-217, 101 p.

Scribner, E.A., Battaglin, W.A., Goolsby, D.A., and Thurman, E.M., 2000, Changes in herbicide concentrations in midwestern streams in relation to changes in use: The Science of the Total Environment, v. 248, p. 255-263.

Scribner, E.A, Goolsby, D.A., Thurman, E.M., and Battaglin, W.A., 1998, A reconnaissance for selected herbicides, degradation products, and nutrients in streams of nine Midwestern States, 1994-95: U.S. Geological Survey Open-File Report 98-141, 44 p.

Scribner, E.A., Goolsby, D.A., Thurman, E.M., Meyer, M.T., and Battaglin, W.A., 1996, Concentrations of selected herbicides, herbicide degradation products, and nutrients in outflow from selected Midwestern reservoirs, April 1992 through September 1993: U.S. Geological Survey Open-File Report 96-393, 128 p. 


\section{Concentrations of Selected Herbicides, Herbicide Degradation Products, and Nutrients, Lower Mississippi River}

Scribner, E.A., Goolsby, D.A., Thurman, E.M., Meyer, M.T., and Pomes, M.L., 1994, Concentrations of selected herbicides, two triazine degradation products, and nutrients in storm runoff from nine stream basins in the Midwestern United States, 1990-92: U.S. Geological Survey Open-File Report 94-396, 144 p.

Scribner, E.A., Thurman, E.M., Goolsby, D.A., Meyer, M.T., Battaglin, W.A., and Kolpin, D.W., 2005, Summary of significant results from studies of triazine herbicides and their degradation products in surface water, ground water, and precipitation in the Midwestern United States during the 1990s: U.S. Geological Survey Scientific Investigations Report 2005-3084, 69 p.

Scribner, E.A., Thurman, E.M., Goolsby, D.A., Meyer, M.T., Mills, M.S., and Pomes, M.L., 1993, Reconnaissance data for selected herbicides, two atrazine degradation products, and nitrate in surface water of the Midwestern United States, 1989-90: U.S. Geological Survey Open-File Report 93-457, $77 \mathrm{p}$.

Thurman, E.M., Goolsby, D.A., Meyer, M.T., and Kolpin, D.W., 1991, Herbicides in surface waters of the Midwestern United States - the effect of spring flush: Environmental Science \& Technology, v. 25, p. 1794-1796.

Thurman, E.M., Goolsby, D.A., Meyer, M.T., Mills, M.S., Pomes, M.L., and Kolpin, D.W., 1992, A reconnaissance study of herbicides and their degradation products in surface water of the Midwestern United States using immunoassay and gas chromatography/mass spectrometry: Environmental Science \& Technology, v. 26, no. 12, p. 2440-2447.
Thurman, E.M., Meyer, M.T., Pomes, M.L., Perry, C.A., and Schwab, A.P., 1990, Enzyme-linked immunosorbent assay compared with gas chromatography/mass spectrometry for the determination of triazine herbicides in water: Analytical Chemistry, v. 62, p. 2043-2048.

Zaugg, S.D., Sandstrom, M.W., Smith, S.G., and Fehlberg, K.M., 1995, Methods of analysis by the U.S. Geological Survey National Water Quality Laboratory-determination of pesticides in water by $\mathrm{C}-18$ solid-phase extraction and capillary-column gas chromatography/mass spectrometry with selected-ion monitoring: U.S. Geological Survey Open-File Report 95-181, 60 p.

Zimmerman, L.R., Hostetler, K.A., and Thurman, E.M., 2000, Methods of analysis by the U.S. Geological Survey Organic Geochemistry Research Group-determination of chloroacetanilide herbicide degradation products in water using highperformance liquid chromatography-diode array detection and high-performance liquid chromatography/mass spectrometry: U.S. Geological Survey Open-File Report 00-182, $30 \mathrm{p}$.

Zimmerman, L.R., and Thurman, E.M., 1999, Method of analysis by the U.S. Geological Survey Organic Geochemistry Research Group - determination of triazine and chloroacetanilide herbicides in water by solid-phase extraction and capillary-column gas chromatography/mass spectrometry with selected-ion monitoring: U.S. Geological Survey Open-File Report 98-634, 21 p. 
Data Tables 
Table 1. Herbicides and their degradation products, years sampled, and method of analysis for water samples collected from the lower Mississippi River in Louisiana, 1991-2003.

[1379, National Water Quality Laboratory method (NWQL) number, 1991-94; 2001, NWQL method number, 1995-2003; GCR, rice and cotton method analysis code, 1996-2003; GCS, corn and soybean method analysis code, 1993-96; HPAA, acetamide degradation products method analysis code, 1998-2001; IME, alachlor immunoassay method analysis code, 1993-98; LCAA, acetamide degradation products method analysis code, 2001-02; LCEA, triazine and phenylurea herbicides and degradation products method analysis code, 1998-2003; LCPD, acetamide parents and degradation products method analysis code, 2002-03; LCGY, glyphosate, AMPA, and glufosinate method analysis code, 2001-03]

\begin{tabular}{|c|c|c|c|}
\hline Herbicide or degradation product & Type of herbicide & Years sampled & Method analysis code \\
\hline Acetochlor & acetamide & 1994-2003 & 1379/2001/GCR/GCS/LCPD \\
\hline Acetochlor ethanesulfonic acid (ESA) & acetamide degradation product & 1998-2003 & HPAA/LCAA/LCPD \\
\hline Acetochlor oxanilic acid (OXA) & acetamide degradation product & 1998-2003 & HPAA/LCAA/LCPD \\
\hline Acetochlor sulfynil acetic acid (SAA) & acetamide degradation product & 2001-2003 & LCAA/LCPD \\
\hline Alachlor & acetamide & $1991-2003$ & 1379/2001/GCR/GCS/LCPD \\
\hline Alachlor ESA by immunoassay & acetamide & 1993-98 & IME \\
\hline AlachlorESA & acetamide & 1998-2003 & HPAA/LCAA/LCPD \\
\hline Alachlor OXA & acetamide & 1998-2003 & HPAA/LCAA/LCPD \\
\hline Alachlor SAA & acetamide & $2001-2003$ & LCAA/LCPD \\
\hline Alachlor ESA 2nd amide & acetamide degradation product & $2002-2003$ & LCPD \\
\hline Ametryn & triazine & $1991-96$ & 1379/2001/GCS \\
\hline Aminomethylphosphonic acid (AMPA) & not assigned degradation product & $2001-2003$ & LCGY \\
\hline Atrazine & triazine & 1991-2003 & 1379/2001/GCR/GCS/LCEA \\
\hline Bromacil & uracil & 2003 & LCEA \\
\hline Cyanazine & triazine & $1991-2003$ & 1379/2001/GCR/GCS/LCEA \\
\hline Cyanazine acid & triazine degradation product & 1998-2003 & LCEA \\
\hline Cyanazine amide & triazine degradation product & 1993-2003 & GCR/GCS/LCEA \\
\hline Deethylatrazine & triazine degradation product & $1991-2003$ & 1379/2001/GCR/GCS/LCEA \\
\hline Deethylcyanazine & triazine degradation product & 1998-2003 & LCEA \\
\hline Deethylcyanazine acid & triazine degradation product & 1998-2003 & LCEA \\
\hline Deethylcyanazine amide & triazine degradation product & 1998-2003 & LCEA \\
\hline Deethylhydroxyatrazine & triazine degradation product & 1998-2003 & LCEA \\
\hline Deisopropylatrazine & triazine degradation product & 1991-2003 & 1379/2001/GCR/GCS/LCEA \\
\hline Deisopropylhydroxyatrazine & triazine degradation product & 1998-2003 & LCEA \\
\hline Deisopropylprometryn & triazine degradation product & 1996-2003 & GCR \\
\hline Demethylfluometuron (DMFM) & phenylurea degradation product & $1995-2003$ & GCR/LCEA \\
\hline Demethylnorflurazon & phenylurea degradation product & $1995-2003$ & GCR \\
\hline Didealkyatrazine (DDA) & phenylurea degradation product & 1998-2003 & LCEA \\
\hline Dimethenamid & acetamide & 1999-2003 & GCR/LCPD \\
\hline Dimethenamid ESA & acetamide degradation product & 1999-2003 & LCAA/LCPD \\
\hline Dimethenamid OXA & acetamide degradation product & 1999-2003 & LCAA/LCPD \\
\hline Diuron & phenylurea & 1998-2003 & LCEA \\
\hline Flufenacet & acetamide & 1999-2003 & GCR/LCPD \\
\hline Flufenacet ESA & acetamide degradation product & $2002-2003$ & LCPD \\
\hline Flufenacet OXA & acetamide degradation product & $2002-2003$ & LCPD \\
\hline
\end{tabular}


Table 1. Herbicides and their degradation products, years sampled, and method of analysis for water samples collected from the lower Mississippi River in Louisiana, 1991-2003.-Continued

[1379, National Water Quality Laboratory method (NWQL) number, 1991-94; 2001, NWQL method number, 1995-2003; GCR, rice and cotton method analysis code, 1996-2003; GCS, corn and soybean method analysis code, 1993-96; HPAA, acetamide degradation products method analysis code, 1998-2001; IME, alachlor immunoassay method analysis code, 1993-98; LCAA, acetamide degradation products method analysis code, 2001-02; LCEA, triazine and phenylurea herbicides and degradation products method analysis code, 1998-2003; LCPD, acetamide parents and degradation products method analysis code, 2002-03; LCGY, glyphosate, AMPA, and glufosinate method analysis code, 2001-03]

\begin{tabular}{|c|c|c|c|}
\hline Herbicide or degradation product & Type of herbicide & Years sampled & Method analysis code \\
\hline Fluometuron & phenylurea & $1995-2003$ & GCR/LCEA \\
\hline Glufosinate & not assigned & $2001-2003$ & LCGY \\
\hline Glyphosate & not assigned & $2001-2003$ & LCGY \\
\hline Hydroxyatrazine & triazine degradation product & $1998-2003$ & LCEA \\
\hline Hydroxysimazine & triazine degradation product & 2003 & LCEA \\
\hline Linuron & phenylurea & $1998-2003$ & LCEA \\
\hline Metolachlor & acetamide & $1991-2003$ & 1379/2001/GCR/GCS/LCPD \\
\hline Metolachlor ESA & acetamide degradation product & 1998-2003 & HPAA/LCAA/LCPD \\
\hline Metolachor OXA & acetamide degradation product & 1998-2003 & HPAA/LCAA/LCPD \\
\hline Metolachlor ESA/Acetochlor 2nd amide & acetamide degradation product & $2002-2003$ & LCPD \\
\hline Metribuzin & triazine & $1991-2003$ & 1379/2001/GCR/GCS \\
\hline Molinate & thiocarbamate & $1995-2003$ & GCR \\
\hline Norflurazon & pyridazinone & 1995-2003 & GCR \\
\hline Pendimethalin & dinitroaniline & 1996-2003 & GCR/GCS \\
\hline Prometon & triazine & $1991-96$ & 1379/2001/GCS \\
\hline Prometryn & triazine & 1991-2003 & 1379/2001/GCR/GCS \\
\hline Propachlor & acetamide & 1995-2003 & 1379/2001/GCS/LCPD \\
\hline Propachlor ESA & acetamide degradation product & 2001-2003 & LCAA/LCPD \\
\hline Propachlor OXA & acetamide degradation product & $2002-2003$ & LCPD \\
\hline Propanil & acetamide & 1995-2003 & GCR \\
\hline Propazine & triazine & 1991-2003 & 1379/2001/GCR/GCS/LCEA \\
\hline Simazine & triazine & 1991-2003 & 1379/2001/GCR/GCS/LCEA \\
\hline Terbutryn & triazine & 1993-96 & GCS \\
\hline 3-trifluomethylaniline (TFMA) & phenylurea degradation product & 1994-2003 & GCR \\
\hline 3-trifluromethylphenylurea (TFMPU) & phenylurea degradation product & 1995-2003 & GCR \\
\hline Trifluralin & dinitroaniline & $1991-2003$ & 1379/2001/GCR \\
\hline
\end{tabular}


Table 2. Results of analysis of selected herbicides and degradation products by U.S. Geological Survey National Water-Quality Laboratory, Lakewood, Colorado, for water samples collected from the Mississippi River near St. Francisville and at Baton Rouge, 1991-2003.

[--, no data; <, less than; *, reported estimates from laboratory; ametryn, molinate, pendamethalin, propanil, propazine, and trifluralin were not detected]

\begin{tabular}{|c|c|c|c|c|c|c|c|c|c|c|c|c|}
\hline \multirow{2}{*}{$\begin{array}{l}\text { Date of } \\
\text { collec- } \\
\text { tion } \\
\text { (month/ } \\
\text { day/year) }\end{array}$} & \multicolumn{12}{|c|}{ Concentration, in micrograms per liter } \\
\hline & Acetochlor & Alachlor & Atrazine & $\begin{array}{l}\text { Cyana- } \\
\text { zine }\end{array}$ & $\begin{array}{l}\text { Deethyl- } \\
\text { atrazine }\end{array}$ & $\begin{array}{l}\text { Deiso- } \\
\text { propyl- } \\
\text { atrazine }\end{array}$ & $\begin{array}{l}\text { Metol- } \\
\text { achlor }\end{array}$ & Metribuzin & Prometon & Prometryn & Propachlor & Simazine \\
\hline \multicolumn{13}{|c|}{ Mississippi River near St. Francisville, Louisiana, station 07373420 (fig. 1) } \\
\hline 05/06/91 & -- & 0.11 & 0.70 & $<0.20$ & 0.13 & $<0.05$ & 0.24 & $<0.05$ & $<0.05$ & $<0.05$ & -- & 0.10 \\
\hline 06/17/91 & -- & .22 & 1.8 & .90 & .14 & .05 & .76 & $<.05$ & $<.05$ & .05 & -- & $<.05$ \\
\hline 07/23/91 & -- & .10 & .72 & .40 & .12 & .05 & .36 & $<.05$ & $<.05$ & .05 & -- & $<.05$ \\
\hline $11 / 05 / 91$ & -- & $<.05$ & .17 & $<.20$ & .06 & .05 & $<.05$ & $<.05$ & $<.05$ & .05 & -- & $<.05$ \\
\hline $11 / 19 / 91$ & -- & $<.05$ & .13 & $<.20$ & .05 & .05 & .05 & $<.05$ & $<.05$ & .05 & -- & $<.05$ \\
\hline $12 / 10 / 91$ & -- & $<.05$ & .11 & $<.20$ & .05 & .05 & .05 & $<.05$ & $<.05$ & .05 & -- & .05 \\
\hline 01/28/92 & -- & $<.05$ & .08 & $<.20$ & $<.05$ & .05 & $<.05$ & $<.05$ & $<.05$ & .05 & -- & $<.05$ \\
\hline $02 / 26 / 92$ & -- & $<.05$ & .11 & -- & -- & -- & .07 & $<.05$ & -- & -- & -- & -- \\
\hline $03 / 25 / 92$ & -- & $<.05$ & .15 & -- & -- & -- & .09 & $<.05$ & -- & -- & -- & -- \\
\hline 05/14/92 & -- & .06 & .90 & .20 & .13 & .05 & .35 & $<.05$ & $<.05$ & .05 & -- & .12 \\
\hline $05 / 26 / 92$ & -- & $<.05$ & .43 & $<.20$ & .10 & .05 & .19 & $<.05$ & $<.05$ & .05 & -- & .08 \\
\hline $06 / 17 / 92$ & -- & .11 & .98 & .40 & .15 & .05 & .46 & .12 & $<.05$ & .05 & -- & .12 \\
\hline 07/15/92 & -- & .13 & 1.2 & .40 & .23 & .05 & .39 & $<.05$ & $<.05$ & .05 & -- & .09 \\
\hline 08/18/92 & -- & .06 & .88 & .20 & .30 & .05 & .24 & .08 & .06 & .05 & -- & $<.05$ \\
\hline 09/15/92 & -- & $<.05$ & .36 & $<.20$ & $<.05$ & .05 & .08 & $<.05$ & $<.05$ & .05 & -- & $<.05$ \\
\hline $01 / 24 / 94$ & -- & $<.05$ & .08 & $<.20$ & $<.05$ & .05 & $<.05$ & $<.05$ & $<.05$ & .05 & -- & .05 \\
\hline 03/14/94 & -- & $<.05$ & .10 & $<.20$ & $<.05$ & .05 & $<.05$ & $<.05$ & $<.05$ & .05 & -- & $<.05$ \\
\hline $06 / 28 / 94$ & -- & $<.05$ & .73 & .30 & .11 & .05 & .17 & $<.05$ & $<.05$ & .05 & -- & .10 \\
\hline 08/30/94 & -- & $<.05$ & .26 & 1.2 & .09 & .05 & $<.05$ & $<.05$ & $<.05$ & .05 & -- & $<.05$ \\
\hline $10 / 20 / 94$ & -- & $<.05$ & .05 & $<.20$ & $<.05$ & .05 & $<.05$ & $<.05$ & $<.05$ & .05 & -- & $<.05$ \\
\hline
\end{tabular}


Table 2. Results of analysis of selected herbicides and degradation products by U.S. Geological Survey National Water-Quality Laboratory, Lakewood, Colorado, for water samples collected from the Mississippi River near St. Francisville and at Baton Rouge, 1991-2003.-Continued

[--, no data; <, less than; *, reported estimates from laboratory; ametryn, molinate, pendamethalin, propanil, propazine, and trifluralin were not detected]

\begin{tabular}{|c|c|c|c|c|c|c|c|c|c|c|c|c|}
\hline \multirow{2}{*}{$\begin{array}{c}\text { Date of } \\
\text { collec- } \\
\text { tion } \\
\text { (month/ } \\
\text { day/year) }\end{array}$} & \multicolumn{12}{|c|}{ Concentration, in micrograms per liter } \\
\hline & Acetochlor & Alachlor & Atrazine & $\begin{array}{l}\text { Cyana- } \\
\text { zine }\end{array}$ & $\begin{array}{l}\text { Deethyl- } \\
\text { atrazine }\end{array}$ & $\begin{array}{l}\text { Deiso- } \\
\text { propyl- } \\
\text { atrazine }\end{array}$ & $\begin{array}{l}\text { Metol- } \\
\text { achlor }\end{array}$ & Metribuzin & Prometon & Prometryn & Propachlor & Simazine \\
\hline \multicolumn{13}{|c|}{ Mississippi River near St. Francisville, Louisiana, station 07373420 (fig. 1)—Continued } \\
\hline $01 / 23 / 95$ & -- & $<0.05$ & 0.06 & $<0.20$ & $<0.05$ & $<0.05$ & $<0.05$ & $<0.05$ & $<0.05$ & $<0.05$ & -- & $<0.05$ \\
\hline 02/21/95 & -- & $<.05$ & .09 & $<.20$ & $<.05$ & $<.05$ & $<.05$ & $<.05$ & $<.05$ & $<.05$ & -- & $<.05$ \\
\hline $04 / 24 / 95$ & -- & $<.05$ & .07 & $<.20$ & $<.05$ & $<.05$ & .09 & $<.05$ & $<.05$ & $<.05$ & -- & $<.05$ \\
\hline 06/14/95 & -- & $<.05$ & 1.8 & .90 & .14 & $<.05$ & .47 & $<.05$ & $<.05$ & $<.05$ & -- & .12 \\
\hline 08/07/95 & -- & $<.05$ & .91 & .30 & .14 & $<.05$ & .22 & $<.05$ & $<.05$ & $<.05$ & -- & $<.05$ \\
\hline $10 / 12 / 95$ & 0.01 & .01 & .19 & $* .05$ & $* .02$ & $<.05$ & .08 & $<.01$ & $* .01$ & $<.05$ & $<0.01$ & .02 \\
\hline $10 / 17 / 95$ & -- & $<.05$ & .06 & $<.20$ & $<.05$ & $<.05$ & .05 & $<.05$ & $<.05$ & $<.05$ & -- & $<.05$ \\
\hline $12 / 06 / 95$ & .01 & .01 & .17 & .03 & $* .02$ & $<.05$ & .09 & $<.01$ & $* .01$ & $<.05$ & $<.01$ & .03 \\
\hline 02/07/96 & .01 & .01 & .06 & .02 & $* .01$ & $<.05$ & .03 & $<.01$ & $* .01$ & $<.05$ & $<.01$ & .02 \\
\hline 03/27/96 & .03 & $<.01$ & .12 & .04 & $* .02$ & -- & .11 & $<.01$ & $<.01$ & -- & $<.01$ & .02 \\
\hline 04/16/96 & .02 & $<.05$ & .07 & $<.20$ & $<.05$ & $<.05$ & .08 & $<.05$ & $<.05$ & $<.05$ & $<.01$ & $<.05$ \\
\hline 05/01/96 & .07 & .05 & 1.1 & .07 & $* .02$ & -- & .49 & .01 & $* .01$ & -- & $<.01$ & .09 \\
\hline 05/17/96 & .16 & .04 & 2.1 & .25 & $* .06$ & -- & .62 & .02 & $* .01$ & -- & $<.01$ & .22 \\
\hline 05/31/96 & .37 & .05 & 3.9 & .88 & $* .10$ & -- & 1.3 & .02 & $* .01$ & -- & $<.01$ & .14 \\
\hline 06/11/96 & .24 & .08 & 3.7 & .92 & $* .11$ & -- & 1.2 & .03 & $* .01$ & -- & $<.01$ & .15 \\
\hline $06 / 21 / 96$ & .36 & .12 & 4.4 & .86 & $* .16$ & -- & 1.8 & .05 & $* .01$ & -- & $<.01$ & .25 \\
\hline 06/25/96 & .37 & .12 & 3.5 & .85 & $* .19$ & -- & 1.4 & .04 & $* .01$ & -- & $<.01$ & .27 \\
\hline 07/23/96 & .15 & .17 & 1.5 & .23 & $* .16$ & .08 & .72 & .11 & .14 & $<.05$ & .13 & .18 \\
\hline 09/03/96 & .01 & .01 & .37 & .06 & $* .05$ & -- & .14 & $<.01$ & $* .01$ & -- & $<.01$ & .02 \\
\hline 09/17/96 & $<.01$ & $* .01$ & .29 & .04 & $* .04$ & $<.05$ & .11 & $<.01$ & $* .01$ & $<.05$ & $<.01$ & .01 \\
\hline $11 / 25 / 96$ & $<.01$ & $<.01$ & .16 & .01 & $* .01$ & .10 & .04 & $<.01$ & $* .01$ & $<.05$ & $<.01$ & .03 \\
\hline
\end{tabular}


Table 2. Results of analysis of selected herbicides and degradation products by U.S. Geological Survey National Water-Quality Laboratory, Lakewood, Colorado, for water samples collected from the Mississippi River near St. Francisville and at Baton Rouge, 1991-2003.-Continued

[--, no data; <, less than; *, reported estimates from laboratory; ametryn, molinate, pendamethalin, propanil, propazine, and trifluralin were not detected]

\begin{tabular}{|c|c|c|c|c|c|c|c|c|c|c|c|c|}
\hline \multirow{2}{*}{$\begin{array}{c}\text { Date of } \\
\text { collec- } \\
\text { tion } \\
\text { (month/ } \\
\text { day/year) }\end{array}$} & \multicolumn{12}{|c|}{ Concentration, in micrograms per liter } \\
\hline & Acetochlor & Alachlor & Atrazine & $\begin{array}{c}\text { Cyana- } \\
\text { zine }\end{array}$ & $\begin{array}{l}\text { Deethyl- } \\
\text { atrazine }\end{array}$ & $\begin{array}{l}\text { Deiso- } \\
\text { propyl- } \\
\text { atrazine }\end{array}$ & $\begin{array}{l}\text { Metol- } \\
\text { achlor }\end{array}$ & Metribuzin & Prometon & Prometryn & Propachlor & Simazine \\
\hline \multicolumn{13}{|c|}{ Mississippi River near St. Francisville, Louisiana, station 07373420 (fig. 1)—Continued } \\
\hline $01 / 15 / 97$ & 0.01 & 0.01 & 0.10 & 0.03 & $* 0.04$ & -- & 0.05 & $<0.01$ & $<0.02$ & -- & $<0.01$ & 0.02 \\
\hline 02/03/97 & .02 & .01 & .09 & .02 & $* .02$ & -- & .05 & $<.01$ & $<.01$ & -- & $<.01$ & .02 \\
\hline 02/20/97 & .01 & .01 & .08 & .02 & $* .01$ & -- & .07 & $<.01$ & $* .01$ & -- & $<.01$ & .02 \\
\hline 03/14/97 & .01 & .01 & .10 & .02 & $* .03$ & -- & .09 & $<.01$ & $* .01$ & -- & $<.01$ & .02 \\
\hline 03/26/97 & .01 & $<.01$ & .07 & .01 & $* .01$ & -- & .07 & $<.01$ & $<.01$ & -- & $<.01$ & .01 \\
\hline 04/08/97 & .01 & .01 & .09 & .01 & $* .01$ & -- & .11 & $<.01$ & $<.01$ & -- & $<.01$ & .01 \\
\hline $04 / 24 / 97$ & .08 & .04 & .73 & .06 & $* .03$ & -- & .34 & $<.01$ & $* .01$ & -- & $<.01$ & .06 \\
\hline 05/06/97 & .12 & .04 & 1.4 & .10 & $* .05$ & -- & .57 & .07 & $* .01$ & -- & $<.01$ & .05 \\
\hline 06/09/97 & .29 & .11 & 2.3 & .29 & $* .09$ & -- & .99 & .05 & $* .01$ & -- & $<.01$ & .14 \\
\hline 06/19/97 & .49 & .17 & 4.7 & .74 & $* .17$ & -- & 2.4 & .08 & $* .01$ & -- & $<.01$ & .41 \\
\hline 07/09/97 & .07 & .03 & 1.5 & .27 & $* .09$ & -- & .57 & .02 & $* .01$ & -- & $<.01$ & .10 \\
\hline 08/04/97 & .02 & .01 & .57 & .08 & $* .05$ & -- & .21 & .01 & $* .01$ & -- & $<.01$ & .03 \\
\hline 08/26/97 & .01 & .01 & .34 & .06 & $* .05$ & -- & .14 & $<.01$ & $* .01$ & -- & $<.01$ & .03 \\
\hline 09/23/97 & .01 & .01 & .23 & $<.01$ & $* .02$ & -- & .07 & $<.01$ & $* .02$ & -- & $<.01$ & .02 \\
\hline $11 / 17 / 97$ & .01 & .01 & .12 & .01 & $* .01$ & -- & .05 & $<.01$ & $* .01$ & -- & $<.01$ & .02 \\
\hline $12 / 16 / 97$ & $<.01$ & .01 & .12 & .02 & $* .03$ & -- & .04 & $<.01$ & $* .01$ & -- & $<.01$ & .02 \\
\hline 01/28/98 & .01 & $<.01$ & .12 & .02 & $* .02$ & -- & .05 & $<.01$ & $<.01$ & -- & $<.01$ & .02 \\
\hline $02 / 27 / 98$ & .01 & $<.01$ & .07 & .01 & $* .02$ & -- & .03 & $<.01$ & $<.01$ & -- & $<.01$ & .02 \\
\hline 03/18/98 & .01 & $<.01$ & .08 & .02 & $* .02$ & -- & .06 & $<.01$ & $<.01$ & -- & $<.01$ & .02 \\
\hline $04 / 30 / 98$ & .07 & .01 & .62 & .06 & $* .02$ & -- & .23 & .01 & $* .01$ & -- & $<.01$ & .09 \\
\hline 05/13/98 & .19 & .03 & 1.6 & .16 & $* .05$ & -- & .52 & .01 & $* .01$ & -- & $<.01$ & .12 \\
\hline 05/28/98 & .21 & .03 & 1.9 & .29 & $* .10$ & -- & .86 & .02 & $* .01$ & -- & $<.01$ & .14 \\
\hline
\end{tabular}


Table 2. Results of analysis of selected herbicides and degradation products by U.S. Geological Survey National Water-Quality Laboratory, Lakewood, Colorado, for water samples collected from the Mississippi River near St. Francisville and at Baton Rouge, 1991-2003.—Continued

[--, no data; <, less than; *, reported estimates from laboratory; ametryn, molinate, pendamethalin, propanil, propazine, and trifluralin were not detected]

\begin{tabular}{|c|c|c|c|c|c|c|c|c|c|c|c|c|}
\hline \multirow{2}{*}{$\begin{array}{c}\text { Date of } \\
\text { collec- } \\
\text { tion } \\
\text { (month/ } \\
\text { day/year) }\end{array}$} & \multicolumn{12}{|c|}{ Concentration, in micrograms per liter } \\
\hline & Acetochlor & Alachlor & Atrazine & $\begin{array}{l}\text { Cyana- } \\
\text { zine }\end{array}$ & $\begin{array}{l}\text { Deethyl- } \\
\text { atrazine }\end{array}$ & $\begin{array}{l}\text { Deiso- } \\
\text { propyl- } \\
\text { atrazine }\end{array}$ & $\begin{array}{l}\text { Metol- } \\
\text { achlor }\end{array}$ & Metribuzin & Prometon & Prometryn & Propachlor & Simazine \\
\hline \multicolumn{13}{|c|}{ Mississippi River near St. Francisville, Louisiana, station 07373420 (fig. 1)—Continued } \\
\hline $06 / 09 / 98$ & 0.56 & 0.05 & 1.9 & 0.38 & $* 0.12$ & -- & 1.4 & 0.04 & $* 0.01$ & -- & $<0.01$ & 0.12 \\
\hline $06 / 25 / 98$ & .22 & .05 & 1.1 & .19 & $* .27$ & -- & .86 & .03 & .02 & -- & $<.01$ & .09 \\
\hline $06 / 30 / 98$ & .21 & .04 & .90 & .15 & $* .19$ & -- & .78 & .02 & $* .01$ & -- & $<.01$ & .08 \\
\hline 07/09/98 & .19 & .04 & .90 & .13 & $* .45$ & -- & 1.1 & .03 & $* .01$ & -- & $<.01$ & .07 \\
\hline 08/19/98 & .01 & .01 & .39 & .06 & $* .08$ & -- & .19 & $<.01$ & $* .01$ & -- & $<.01$ & .02 \\
\hline $09 / 28 / 98$ & $<.01$ & $<.01$ & .24 & .03 & $* .07$ & -- & .06 & $<.01$ & $* .01$ & -- & $<.01$ & .02 \\
\hline $11 / 18 / 98$ & $<.01$ & $<.01$ & .15 & $<.01$ & $* .04$ & -- & .05 & $<.01$ & $<.02$ & -- & $<.01$ & .02 \\
\hline $12 / 08 / 98$ & .01 & $<.01$ & .16 & .02 & $* .05$ & -- & .07 & $<.01$ & $<.01$ & -- & $<.01$ & .01 \\
\hline $01 / 12 / 99$ & .01 & $<.01$ & .08 & .01 & $* .02$ & -- & .04 & $<.01$ & $<.01$ & -- & $<.01$ & .03 \\
\hline 02/10/99 & .01 & $<.01$ & .08 & .01 & $* .02$ & -- & .04 & $<.01$ & $<.01$ & -- & $<.01$ & .02 \\
\hline 03/15/99 & .01 & $<.01$ & .08 & .01 & $* .02$ & -- & .03 & $<.01$ & $<.01$ & -- & $<.01$ & .02 \\
\hline 03/30/99 & .01 & $<.01$ & .11 & .01 & $* .01$ & -- & .04 & $<.01$ & $* .01$ & -- & $<.01$ & .02 \\
\hline 04/19/99 & .02 & .01 & .84 & .03 & $* .03$ & -- & .16 & .01 & $<.01$ & -- & $<.01$ & .28 \\
\hline $04 / 28 / 99$ & .08 & .01 & .72 & .03 & $* .04$ & -- & .21 & .01 & $<.01$ & -- & $<.01$ & .07 \\
\hline $05 / 06 / 99$ & .15 & .01 & 1.3 & .06 & $* .07$ & -- & .46 & .02 & $<.01$ & -- & $<.01$ & .05 \\
\hline $05 / 26 / 99$ & .16 & .02 & 1.8 & .09 & $* .07$ & -- & .46 & .02 & $<.02$ & -- & $<.01$ & .21 \\
\hline $06 / 10 / 99$ & .48 & .03 & .14 & .01 & $* .12$ & -- & .75 & .02 & $* .01$ & -- & $<.01$ & .02 \\
\hline $06 / 24 / 99$ & .32 & .05 & .41 & .03 & $* .27$ & -- & .82 & .03 & $* .01$ & -- & $<.01$ & .04 \\
\hline $07 / 21 / 99$ & .03 & .02 & .80 & .12 & $* .22$ & -- & .36 & .01 & $* .01$ & -- & $<.01$ & .02 \\
\hline 08/05/99 & .03 & .02 & .71 & .06 & $* .14$ & -- & .22 & $<.01$ & $* .01$ & -- & $<.01$ & .02 \\
\hline $09 / 14 / 99$ & $<.01$ & $<.01$ & .21 & .02 & $* .06$ & -- & .05 & $<.01$ & $* .01$ & -- & $<.01$ & .02 \\
\hline $11 / 11 / 99$ & .01 & $<.01$ & .13 & .01 & $* .03$ & -- & .03 & $<.01$ & $* .01$ & -- & $<.01$ & .02 \\
\hline $12 / 01 / 99$ & .02 & $<.01$ & .11 & $<.02$ & $* .03$ & -- & .04 & $<.01$ & $* .01$ & -- & $<.01$ & .05 \\
\hline
\end{tabular}


Table 2. Results of analysis of selected herbicides and degradation products by U.S. Geological Survey National Water-Quality Laboratory, Lakewood, Colorado, for water samples collected from the Mississippi River near St. Francisville and at Baton Rouge, 1991-2003.-Continued

[--, no data; <, less than; *, reported estimates from laboratory; ametryn, molinate, pendamethalin, propanil, propazine, and trifluralin were not detected]

\begin{tabular}{|c|c|c|c|c|c|c|c|c|c|c|c|c|}
\hline \multirow{2}{*}{$\begin{array}{c}\text { Date of } \\
\text { collec- } \\
\text { tion } \\
\text { (month/ } \\
\text { day/year) }\end{array}$} & \multicolumn{12}{|c|}{ Concentration, in micrograms per liter } \\
\hline & Acetochlor & Alachlor & Atrazine & $\begin{array}{l}\text { Cyana- } \\
\text { zine }\end{array}$ & $\begin{array}{l}\text { Deethyl- } \\
\text { atrazine }\end{array}$ & $\begin{array}{l}\text { Deiso- } \\
\text { propyl- } \\
\text { atrazine }\end{array}$ & $\begin{array}{l}\text { Metol- } \\
\text { achlor }\end{array}$ & Metribuzin & Prometon & Prometryn & Propachlor & Simazine \\
\hline \multicolumn{13}{|c|}{ Mississippi River near St. Francisville, Louisiana, station 07373420 (fig. 1)—Continued } \\
\hline $01 / 27 / 00$ & 0.01 & 0.01 & 0.14 & 0.01 & $* 0.04$ & -- & 0.07 & $<0.01$ & $* 0.01$ & -- & $<0.01$ & 0.04 \\
\hline $02 / 17 / 00$ & .01 & .01 & .14 & $<.01$ & $* .04$ & -- & .04 & $<.01$ & $* .01$ & -- & $<.01$ & .02 \\
\hline $03 / 14 / 00$ & .02 & $<.01$ & .14 & $<.01$ & $* .03$ & -- & .07 & .01 & $<.01$ & -- & $<.01$ & .05 \\
\hline 03/29/00 & .02 & $<.01$ & .36 & .01 & $* .04$ & -- & .11 & $<.01$ & $<.01$ & -- & $<.01$ & .10 \\
\hline $04 / 12 / 00$ & .03 & .01 & .56 & .02 & $* .04$ & -- & .17 & $<.01$ & $* .01$ & -- & $<.01$ & .05 \\
\hline 05/10/00 & .07 & .01 & .90 & .03 & $* .06$ & -- & .31 & .02 & $* .01$ & -- & $<.01$ & .08 \\
\hline $05 / 23 / 00$ & .08 & .06 & 1.3 & .02 & $* .11$ & -- & .28 & .02 & $* .01$ & -- & $<.01$ & .09 \\
\hline 06/06/00 & .13 & .04 & 2.0 & .02 & $* .22$ & -- & .45 & .02 & $* .01$ & -- & $<.01$ & .17 \\
\hline $06 / 23 / 00$ & .21 & .02 & 2.0 & .03 & $* .20$ & -- & .61 & .02 & $* .02$ & -- & $<.01$ & .06 \\
\hline 07/18/00 & .07 & .01 & 1.1 & .02 & $* .25$ & -- & .33 & .01 & $* .01$ & -- & $<.01$ & .05 \\
\hline $08 / 25 / 00$ & .01 & .01 & .24 & .02 & $* .07$ & -- & .06 & $<.01$ & $* .02$ & -- & $<.01$ & .03 \\
\hline $09 / 21 / 00$ & $<.01$ & $<.01$ & .15 & .01 & $* .06$ & -- & .03 & $<.01$ & $* .01$ & -- & $<.01$ & .01 \\
\hline $11 / 28 / 00$ & $<.01$ & $<.01$ & .14 & $* .01$ & $* .04$ & -- & .04 & $<.01$ & $* .01$ & -- & $<.01$ & .06 \\
\hline $12 / 14 / 00$ & .01 & $<.01$ & .09 & $<.01$ & $* .03$ & -- & .02 & $<.01$ & $<.01$ & -- & $<.01$ & .05 \\
\hline $01 / 24 / 01$ & $<.01$ & $<.01$ & .09 & $<.01$ & $* .02$ & -- & .03 & $<.01$ & $<.01$ & -- & $<.01$ & .04 \\
\hline $02 / 27 / 01$ & $<.01$ & $<.01$ & .08 & $<.01$ & $* .02$ & -- & .05 & $<.01$ & $<.01$ & -- & $<.01$ & .10 \\
\hline 03/14/01 & $<.01$ & $<.01$ & .09 & $<.01$ & $* .02$ & -- & .06 & $<.01$ & $<.01$ & -- & $<.01$ & .06 \\
\hline 04/02/01 & .03 & $<.01$ & .11 & $<.01$ & $* .02$ & -- & .06 & $<.01$ & $<.01$ & -- & $<.01$ & .03 \\
\hline $04 / 16 / 01$ & .01 & $<.01$ & .41 & $* .01$ & $* .02$ & -- & .31 & $<.01$ & $<.01$ & -- & $<.01$ & .09 \\
\hline $04 / 23 / 01$ & .04 & $<.01$ & .42 & $* .01$ & $* .03$ & -- & .27 & .01 & $<.01$ & -- & $<.01$ & .05 \\
\hline 05/07/01 & .05 & .01 & .39 & $<.01$ & $* .04$ & -- & .24 & .01 & *.01 & -- & $<.01$ & .04 \\
\hline 05/21/01 & .19 & .01 & 1.0 & $<.01$ & $* .04$ & -- & .42 & .01 & $<.01$ & -- & $<.01$ & .06 \\
\hline
\end{tabular}


Table 2. Results of analysis of selected herbicides and degradation products by U.S. Geological Survey National Water-Quality Laboratory, Lakewood, Colorado, for water samples collected from the Mississippi River near St. Francisville and at Baton Rouge, 1991-2003.—Continued

[--, no data; <, less than; *, reported estimates from laboratory; ametryn, molinate, pendamethalin, propanil, propazine, and trifluralin were not detected]

\begin{tabular}{|c|c|c|c|c|c|c|c|c|c|c|c|c|}
\hline \multirow{2}{*}{$\begin{array}{c}\text { Date of } \\
\text { collec- } \\
\text { tion } \\
\text { (month/ } \\
\text { day/year) }\end{array}$} & \multicolumn{12}{|c|}{ Concentration, in micrograms per liter } \\
\hline & Acetochlor & Alachlor & Atrazine & $\begin{array}{l}\text { Cyana- } \\
\text { zine }\end{array}$ & $\begin{array}{l}\text { Deethyl- } \\
\text { atrazine }\end{array}$ & $\begin{array}{l}\text { Deiso- } \\
\text { propyl- } \\
\text { atrazine }\end{array}$ & $\begin{array}{l}\text { Metol- } \\
\text { achlor }\end{array}$ & Metribuzin & Prometon & Prometryn & Propachlor & Simazine \\
\hline \multicolumn{13}{|c|}{ Mississippi River near St. Francisville, Louisiana, station 07373420 (fig. 1)—Continued } \\
\hline $06 / 25 / 01$ & 0.19 & 0.02 & 0.61 & 0.02 & $* 0.26$ & -- & 0.50 & 0.01 & $<0.01$ & -- & $<0.01$ & 0.07 \\
\hline 07/10/01 & .16 & .02 & .77 & .02 & $* .14$ & -- & .35 & .01 & $* .01$ & -- & $<.01$ & .04 \\
\hline $07 / 18 / 01$ & .08 & .02 & .75 & .04 & $* .08$ & -- & .26 & $<.01$ & $* .01$ & -- & $<.01$ & .03 \\
\hline $08 / 27 / 01$ & .01 & $<.01$ & .26 & $* .01$ & $* .05$ & -- & .06 & $<.01$ & $* .01$ & -- & $<.01$ & .02 \\
\hline 09/19/01 & .01 & $<.01$ & .16 & $<.01$ & $* .03$ & -- & .04 & $<.01$ & $* .01$ & -- & $<.01$ & .01 \\
\hline $10 / 30 / 01$ & .01 & $<.01$ & .14 & $<.01$ & $* .04$ & -- & .04 & $<.01$ & *.01 & -- & $<.01$ & .05 \\
\hline $12 / 18 / 01$ & $<.01$ & $<.01$ & .08 & $<.01$ & $* .02$ & -- & .02 & $<.01$ & $* .01$ & -- & $<.01$ & .40 \\
\hline $01 / 22 / 02$ & .02 & $<.01$ & .07 & $<.01$ & $* .02$ & -- & .03 & $<.01$ & $<.01$ & -- & $<.01$ & .11 \\
\hline $02 / 04 / 02$ & $<.01$ & $<.01$ & .06 & $<.01$ & $* .02$ & -- & .02 & $<.01$ & $* .01$ & -- & $<.01$ & .08 \\
\hline $02 / 18 / 02$ & .01 & $<.01$ & .09 & $<.01$ & $* .03$ & -- & .03 & $<.01$ & $* .01$ & -- & $<.01$ & .10 \\
\hline 03/13/02 & .03 & $<.01$ & .08 & $<.01$ & $* .01$ & -- & .05 & $<.01$ & $<.01$ & -- & $<.01$ & .06 \\
\hline $03 / 25 / 02$ & .01 & $<.01$ & .23 & $<.01$ & $* .01$ & -- & .07 & $<.01$ & $<.01$ & -- & $<.01$ & .11 \\
\hline $04 / 10 / 02$ & $<.01$ & $<.01$ & .16 & $<.01$ & $* .01$ & -- & .04 & $<.01$ & $<.01$ & -- & $<.01$ & .06 \\
\hline $04 / 22 / 02$ & .01 & .01 & .31 & $<.01$ & $* .01$ & -- & .06 & $<.01$ & $* .01$ & -- & $<.01$ & .05 \\
\hline $05 / 07 / 02$ & .15 & .02 & 2.1 & $<.01$ & $* .06$ & -- & .33 & $<.01$ & $* .01$ & -- & $<.01$ & .25 \\
\hline $05 / 28 / 02$ & .32 & .03 & 2.7 & $* .01$ & $* .14$ & -- & .52 & .01 & $* .01$ & -- & $<.01$ & .25 \\
\hline $06 / 11 / 02$ & .23 & .02 & 1.7 & $<.01$ & $* .14$ & -- & .44 & .01 & $* .01$ & -- & $<.01$ & .16 \\
\hline 08/05/02 & .03 & $<.01$ & .92 & $* .02$ & *.08 & -- & .11 & $<.01$ & $* .01$ & -- & $<.01$ & .05 \\
\hline 08/19/02 & .02 & $<.01$ & .49 & $<.01$ & $* .05$ & -- & .06 & $<.01$ & $* .01$ & -- & $<.01$ & .03 \\
\hline 09/09/02 & .01 & .01 & .33 & $<.01$ & $* .07$ & -- & .05 & $<.01$ & $* .01$ & -- & $<.01$ & .02 \\
\hline $11 / 12 / 02$ & .01 & $<.01$ & .11 & $<.01$ & $* .03$ & -- & .03 & $<.01$ & $* .01$ & -- & $<.01$ & .04 \\
\hline $01 / 14 / 03$ & .01 & $<.01$ & .08 & $<.01$ & $* .03$ & -- & .03 & $* .01$ & $* .01$ & -- & $<.01$ & .28 \\
\hline
\end{tabular}


Table 2. Results of analysis of selected herbicides and degradation products by U.S. Geological Survey National Water-Quality Laboratory, Lakewood, Colorado, for water samples collected from the Mississippi River near St. Francisville and at Baton Rouge, 1991-2003.—Continued

[--, no data; <, less than; *, reported estimates from laboratory; ametryn, molinate, pendamethalin, propanil, propazine, and trifluralin were not detected]

\begin{tabular}{|c|c|c|c|c|c|c|c|c|c|c|c|c|}
\hline \multirow{2}{*}{$\begin{array}{c}\text { Date of } \\
\text { collec- } \\
\text { tion } \\
\text { (month/ } \\
\text { day/year) }\end{array}$} & \multicolumn{12}{|c|}{ Concentration, in micrograms per liter } \\
\hline & Acetochlor & Alachlor & Atrazine & $\begin{array}{l}\text { Cyana- } \\
\text { zine }\end{array}$ & $\begin{array}{l}\text { Deethyl- } \\
\text { atrazine }\end{array}$ & $\begin{array}{l}\text { Deiso- } \\
\text { propyl- } \\
\text { atrazine }\end{array}$ & $\begin{array}{l}\text { Metol- } \\
\text { achlor }\end{array}$ & Metribuzin & Prometon & Prometryn & Propachlor & Simazine \\
\hline \multicolumn{13}{|c|}{ Mississippi River near St. Francisville, Louisiana, station 07373420 (fig. 1) —Continued } \\
\hline $01 / 28 / 03$ & 0.01 & $<0.01$ & 0.06 & $<0.01$ & $* 0.02$ & -- & 0.02 & $<0.01$ & $* 0.01$ & -- & $<0.01$ & 0.09 \\
\hline $02 / 12 / 03$ & $<.01$ & $<.01$ & .06 & $<.01$ & $* .02$ & -- & .02 & .01 & $<.01$ & -- & $<.01$ & .04 \\
\hline $02 / 24 / 03$ & $<.01$ & $<.01$ & .06 & $<.01$ & $* .02$ & -- & .02 & $<.01$ & $<.01$ & -- & $<.01$ & .06 \\
\hline 03/10/03 & $<.01$ & $<.01$ & .05 & $<.01$ & $* .01$ & -- & .02 & $<.01$ & $<.01$ & -- & $<.01$ & .08 \\
\hline 03/24/03 & $<.01$ & $<.01$ & .07 & $<.01$ & $* .02$ & -- & .03 & $<.01$ & $<.01$ & -- & $<.01$ & .05 \\
\hline $04 / 15 / 03$ & .01 & .01 & 1.5 & $<.01$ & $* .04$ & -- & .33 & .01 & $<.01$ & -- & $<.01$ & .09 \\
\hline $04 / 29 / 03$ & .03 & .01 & .73 & $<.01$ & $* .03$ & -- & .13 & $<.01$ & $<.01$ & -- & $<.01$ & .06 \\
\hline $05 / 12 / 03$ & .12 & .02 & 2.8 & $<.01$ & $* .10$ & -- & .40 & .01 & $* .01$ & -- & $<.01$ & .32 \\
\hline $05 / 27 / 03$ & .29 & .02 & 2.2 & $<.01$ & $* .17$ & -- & .45 & .01 & $* .01$ & -- & $<.01$ & .19 \\
\hline 06/16/03 & .12 & .01 & 1.3 & $<.01$ & $* .10$ & -- & .27 & $<.01$ & $* .01$ & -- & $<.01$ & .09 \\
\hline $07 / 14 / 03$ & .07 & .01 & 1.0 & $<.01$ & $* .10$ & -- & .20 & $<.01$ & $* .01$ & -- & $<.01$ & .03 \\
\hline 08/12/03 & .03 & $<.01$ & .35 & $<.01$ & $* .07$ & -- & .11 & $<.01$ & $* .01$ & -- & $<.01$ & .02 \\
\hline 09/23/03 & .01 & $<.01$ & .16 & $<.01$ & $* .04$ & -- & .05 & $<.01$ & $* .01$ & -- & $<.01$ & .02 \\
\hline $10 / 27 / 03$ & .01 & $* .01$ & .13 & $<.01$ & $* .01$ & -- & .03 & $<.01$ & .01 & -- & $<.01$ & .02 \\
\hline $12 / 09 / 03$ & .01 & .01 & .08 & $<.01$ & $* .01$ & -- & .02 & .01 & .01 & -- & $<.01$ & .23 \\
\hline \multicolumn{13}{|c|}{ Mississippi River at Baton Rouge, Louisiana, station 07374000 (fig. 1) } \\
\hline $04 / 11 / 91$ & -- & $<.05$ & .28 & $<.20$ & .05 & $<0.05$ & .12 & $<.05$ & $<.05$ & $<0.05$ & -- & $<.05$ \\
\hline 04/17/91 & -- & $<.05$ & .23 & $<.20$ & $<.05$ & $<.05$ & .10 & $<.05$ & $<.05$ & $<.05$ & -- & $<.05$ \\
\hline $04 / 24 / / 91$ & -- & $<.05$ & .39 & $<.20$ & $<.05$ & $<.05$ & .13 & $<.05$ & $<.05$ & $<.05$ & -- & $<.05$ \\
\hline 05/01/91 & -- & .06 & .52 & .30 & $<.05$ & $<.05$ & .18 & $<.05$ & $<.05$ & $<.05$ & -- & $<.05$ \\
\hline 05/06/91 & -- & $<.05$ & .49 & .21 & .16 & $<.05$ & .18 & $<.05$ & .09 & $<.05$ & -- & .10 \\
\hline 05/09/91 & -- & .13 & 1.0 & .21 & .16 & $<.05$ & .31 & $<.05$ & .09 & $<.05$ & -- & .10 \\
\hline 05/13/91 & -- & .10 & .59 & $<.20$ & .13 & $<.05$ & .23 & $<.05$ & $<.05$ & $<.05$ & -- & .08 \\
\hline 05/16/91 & -- & $<.05$ & .34 & .20 & $<.05$ & $<.05$ & .14 & $<.05$ & $<.05$ & $<.05$ & -- & $<.05$ \\
\hline
\end{tabular}


Table 2. Results of analysis of selected herbicides and degradation products by U.S. Geological Survey National Water-Quality Laboratory, Lakewood, Colorado, for water samples collected from the Mississippi River near St. Francisville and at Baton Rouge, 1991-2003.—Continued

[--, no data; <, less than; *, reported estimates from laboratory; ametryn, molinate, pendamethalin, propanil, propazine, and trifluralin were not detected]

\begin{tabular}{|c|c|c|c|c|c|c|c|c|c|c|c|c|}
\hline \multirow{2}{*}{$\begin{array}{c}\text { Date of } \\
\text { collec- } \\
\text { tion } \\
\text { (month/ } \\
\text { day/year) }\end{array}$} & \multicolumn{12}{|c|}{ Concentration, in micrograms per liter } \\
\hline & Acetochlor & Alachlor & Atrazine & $\begin{array}{l}\text { Cyana- } \\
\text { zine }\end{array}$ & $\begin{array}{l}\text { Deethyl- } \\
\text { atrazine }\end{array}$ & $\begin{array}{l}\text { Deiso- } \\
\text { propyl- } \\
\text { atrazine }\end{array}$ & $\begin{array}{l}\text { Metol- } \\
\text { achlor }\end{array}$ & Metribuzin & Prometon & Prometryn & Propachlor & Simazine \\
\hline \multicolumn{13}{|c|}{ Mississippi River at Baton Rouge, Louisiana, station 07374000 (fig. 1) -Continued } \\
\hline $05 / 20 / 91$ & -- & 0.13 & 1.2 & 0.90 & 0.11 & $<0.05$ & 0.38 & $<0.05$ & $<0.05$ & $<0.05$ & -- & $<0.05$ \\
\hline $05 / 24 / 91$ & -- & .14 & 1.1 & .60 & .10 & $<.05$ & .40 & $<.05$ & $<.05$ & $<.05$ & -- & $<.05$ \\
\hline $05 / 28 / 91$ & -- & .07 & .83 & .30 & .09 & $<.05$ & .36 & $<.05$ & $<.05$ & $<.05$ & -- & $<.05$ \\
\hline $05 / 30 / 91$ & -- & .13 & 1.1 & .40 & .11 & $<.05$ & .36 & .09 & $<.05$ & $<.05$ & -- & .09 \\
\hline 06/03/91 & -- & .18 & 1.6 & .90 & .17 & $<.05$ & .48 & $<.05$ & $<.05$ & $<.05$ & -- & .08 \\
\hline 06/06/91 & -- & .34 & 2.0 & 1.1 & .16 & $<.05$ & .71 & $<.05$ & $<.05$ & $<.05$ & -- & .08 \\
\hline 06/10/91 & -- & .23 & 1.5 & .80 & .10 & $<.05$ & .67 & $<.05$ & $<.05$ & $<.05$ & & .05 \\
\hline $06 / 13 / 91$ & -- & .29 & 2.6 & 1.2 & .29 & $<.05$ & .97 & .09 & $<.05$ & $<.05$ & -- & .11 \\
\hline $06 / 17 / 91$ & -- & .20 & 1.9 & .80 & .16 & $<.05$ & .71 & $<.05$ & $<.05$ & $<.05$ & -- & .05 \\
\hline $06 / 20 / 91$ & -- & .30 & 2.5 & .88 & .22 & $<.05$ & .87 & .20 & $<.05$ & $<.05$ & -- & .07 \\
\hline $06 / 24 / 91$ & -- & .44 & 3.6 & 1.8 & .32 & .19 & 1.4 & .20 & .05 & $<.05$ & -- & .08 \\
\hline $06 / 27 / 91$ & -- & .40 & 2.6 & 1.5 & .25 & $<.05$ & 1.2 & .09 & $<.05$ & $<.05$ & -- & .07 \\
\hline 07/03/91 & -- & .22 & 1.5 & .50 & .18 & $<.05$ & .61 & .10 & $<.05$ & $<.05$ & -- & .06 \\
\hline 07/08/91 & -- & .16 & 1.3 & .50 & .16 & $<.05$ & .57 & .10 & $<.05$ & $<.05$ & -- & .05 \\
\hline $07 / 11 / 91$ & -- & .46 & 3.3 & 1.2 & .39 & .15 & 1.3 & .08 & .08 & $<.05$ & -- & .07 \\
\hline $07 / 17 / 91$ & -- & .16 & 1.1 & .40 & .17 & .10 & .51 & .10 & .13 & .06 & -- & .05 \\
\hline $07 / 23 / 91$ & -- & .07 & .64 & $<.20$ & $<.05$ & $<.05$ & .26 & $<.05$ & $<.05$ & $<.05$ & -- & $<.05$ \\
\hline $07 / 30 / 91$ & -- & .08 & .68 & .30 & .13 & $<.05$ & .25 & $<.05$ & $<.05$ & $<.05$ & -- & $<.05$ \\
\hline 08/06/91 & -- & $<.05$ & .36 & .20 & .07 & $<.05$ & .16 & $<.05$ & $<.05$ & $<.05$ & -- & $<.05$ \\
\hline $08 / 12 / 91$ & -- & .05 & .40 & .20 & $<.05$ & $<.05$ & .20 & $<.05$ & $<.05$ & $<.05$ & -- & $<.05$ \\
\hline 08/20/91 & -- & $<.05$ & .46 & .30 & $<.05$ & $<.05$ & .20 & $<.05$ & $<.05$ & $<.05$ & -- & $<.05$ \\
\hline $08 / 26 / 91$ & -- & $<.05$ & $<.05$ & .25 & .17 & .14 & $<.05$ & $<.05$ & .16 & .07 & -- & .06 \\
\hline
\end{tabular}


Table 2. Results of analysis of selected herbicides and degradation products by U.S. Geological Survey National Water-Quality Laboratory, Lakewood, Colorado, for water samples collected from the Mississippi River near St. Francisville and at Baton Rouge, 1991-2003.—Continued

[--, no data; <, less than; *, reported estimates from laboratory; ametryn, molinate, pendamethalin, propanil, propazine, and trifluralin were not detected]

\begin{tabular}{|c|c|c|c|c|c|c|c|c|c|c|c|c|}
\hline \multirow{2}{*}{$\begin{array}{c}\text { Date of } \\
\text { collec- } \\
\text { tion } \\
\text { (month/ } \\
\text { day/year) }\end{array}$} & \multicolumn{12}{|c|}{ Concentration, in micrograms per liter } \\
\hline & Acetochlor & Alachlor & Atrazine & $\begin{array}{l}\text { Cyana- } \\
\text { zine }\end{array}$ & $\begin{array}{l}\text { Deethyl- } \\
\text { atrazine }\end{array}$ & $\begin{array}{l}\text { Deiso- } \\
\text { propyl- } \\
\text { atrazine }\end{array}$ & $\begin{array}{l}\text { Metol- } \\
\text { achlor }\end{array}$ & Metribuzin & Prometon & Prometryn & Propachlor & Simazine \\
\hline \multicolumn{13}{|c|}{ Mississippi River at Baton Rouge, Louisiana, station 07374000 (fig. 1)—Continued } \\
\hline 09/03/91 & -- & $<0.05$ & $<0.05$ & $<0.20$ & $<0.05$ & $<0.05$ & $<0.05$ & $<0.05$ & $<0.05$ & $<0.05$ & -- & $<0.05$ \\
\hline 09/09/91 & -- & $<.05$ & .26 & .30 & $<.05$ & $<.05$ & .09 & $<.05$ & $<.05$ & $<.05$ & -- & $<.05$ \\
\hline 09/18/91 & -- & $<.05$ & .17 & $<.20$ & $<.05$ & $<.05$ & $<.05$ & $<.05$ & $<.05$ & $<.05$ & -- & $<.05$ \\
\hline 09/23/91 & -- & $<.05$ & .16 & $<.20$ & $<.05$ & $<.05$ & $<.05$ & $<.05$ & $<.05$ & $<.05$ & -- & $<.05$ \\
\hline 10/07/91 & -- & $<.05$ & .15 & $<.20$ & .06 & $<.10$ & $<.05$ & $<.05$ & $<.05$ & $<.05$ & -- & $<.05$ \\
\hline $10 / 16 / 91$ & -- & $<.05$ & .10 & $<.20$ & $<.05$ & $<.05$ & $<.05$ & $<.05$ & $<.05$ & $<.05$ & -- & $<.05$ \\
\hline $10 / 23 / 91$ & -- & $<.05$ & $<.05$ & $<.20$ & $<.05$ & $<.05$ & $<.05$ & $<.05$ & $<.05$ & $<.05$ & -- & $<.05$ \\
\hline $10 / 31 / 91$ & -- & $<.05$ & .27 & .39 & .08 & $<.05$ & .06 & $<.05$ & .06 & $<.05$ & -- & $<.05$ \\
\hline $11 / 07 / 91$ & -- & $<.05$ & .17 & $<.20$ & .06 & $<.05$ & $<.05$ & $<.05$ & $<.05$ & $<.05$ & -- & $<.05$ \\
\hline $11 / 14 / 91$ & -- & $<.05$ & .18 & $<.20$ & .06 & $<.05$ & .05 & $<.05$ & $<.05$ & $<.05$ & -- & $<.05$ \\
\hline $11 / 19 / 91$ & -- & $<.05$ & .13 & $<.20$ & .05 & $<.05$ & .05 & $<.05$ & $<.05$ & $<.05$ & -- & $<.05$ \\
\hline $11 / 25 / 91$ & -- & .05 & .17 & $<.20$ & .06 & $<.05$ & .08 & $<.05$ & $<.05$ & $<.05$ & -- & $<.05$ \\
\hline 12/01/91 & -- & $<.05$ & .12 & $<.20$ & .05 & $<.05$ & .05 & $<.05$ & $<.05$ & $<.05$ & -- & $<.05$ \\
\hline $12 / 10 / 91$ & -- & $<.05$ & .10 & $<.20$ & .05 & $<.05$ & .05 & $<.05$ & $<.05$ & $<.05$ & -- & .07 \\
\hline $12 / 17 / 91$ & -- & $<.05$ & .07 & $<.20$ & $<.05$ & $<.05$ & $<.05$ & $<.05$ & $<.05$ & $<.05$ & -- & $<.05$ \\
\hline $12 / 24 / 91$ & -- & $<.05$ & .09 & $<.20$ & $<.05$ & $<.05$ & .05 & $<.05$ & $<.05$ & $<.05$ & -- & $<.05$ \\
\hline $12 / 31 / 91$ & -- & $<.05$ & .10 & $<.20$ & .08 & $<.05$ & .05 & $<.05$ & $<.05$ & $<.05$ & -- & $<.05$ \\
\hline 01/07/92 & -- & $<.05$ & .10 & $<.20$ & .07 & $<.05$ & .06 & $<.05$ & $<.05$ & $<.05$ & -- & $<.05$ \\
\hline 01/15/92 & -- & $<.05$ & .12 & $<.20$ & .05 & $<.05$ & .07 & $<.05$ & $<.05$ & $<.05$ & -- & $<.05$ \\
\hline $01 / 20 / 92$ & -- & $<.05$ & .11 & $<.20$ & .05 & $<.05$ & .05 & $<.05$ & $<.05$ & $<.05$ & -- & $<.05$ \\
\hline 01/29/92 & -- & $<.05$ & .09 & $<.20$ & .04 & $<.05$ & .05 & $<.05$ & .01 & $<.05$ & -- & $<.05$ \\
\hline 02/06/92 & -- & $<.05$ & $<.05$ & $<.20$ & $<.05$ & $<.05$ & $<.05$ & $<.05$ & $<.05$ & $<.05$ & -- & $<.05$ \\
\hline
\end{tabular}


Table 2. Results of analysis of selected herbicides and degradation products by U.S. Geological Survey National Water-Quality Laboratory, Lakewood, Colorado, for water samples collected from the Mississippi River near St. Francisville and at Baton Rouge, 1991-2003.—Continued

[--, no data; <, less than; *, reported estimates from laboratory; ametryn, molinate, pendamethalin, propanil, propazine, and trifluralin were not detected]

\begin{tabular}{|c|c|c|c|c|c|c|c|c|c|c|c|c|}
\hline \multirow{2}{*}{$\begin{array}{l}\text { Date of } \\
\text { collec- } \\
\text { tion } \\
\text { (month/ } \\
\text { day/year) }\end{array}$} & \multicolumn{12}{|c|}{ Concentration, in micrograms per liter } \\
\hline & Acetochlor & Alachlor & Atrazine & $\begin{array}{c}\text { Cyana- } \\
\text { zine }\end{array}$ & $\begin{array}{l}\text { Deethyl- } \\
\text { atrazine }\end{array}$ & $\begin{array}{l}\text { Deiso- } \\
\text { propyl- } \\
\text { atrazine }\end{array}$ & $\begin{array}{l}\text { Metol- } \\
\text { achlor }\end{array}$ & Metribuzin & Prometon & Prometryn & Propachlor & Simazine \\
\hline \multicolumn{13}{|c|}{ Mississippi River at Baton Rouge, Louisiana, station 07374000 (fig. 1)-Continued } \\
\hline 02/13/92 & -- & 0.05 & 0.13 & $<0.20$ & 0.08 & $<0.05$ & 0.08 & $<0.05$ & $<0.05$ & $<0.05$ & -- & 0.06 \\
\hline 02/20/92 & -- & $<.05$ & $<.10$ & $<.20$ & .07 & $<.05$ & .07 & $<.05$ & $<.05$ & $<.05$ & -- & $<.05$ \\
\hline 02/26/92 & -- & $<.05$ & $<.05$ & $<.20$ & $<.05$ & $<.05$ & $<.05$ & $<.05$ & $<.05$ & $<.05$ & -- & $<.05$ \\
\hline 03/05/92 & -- & $<.05$ & .07 & $<.20$ & $<.05$ & $<.05$ & .05 & $<.05$ & $<.05$ & $<.05$ & -- & $<.05$ \\
\hline 03/11/92 & -- & $<.05$ & .11 & $<.20$ & .07 & $<.05$ & .08 & $<.05$ & $<.05$ & $<.05$ & -- & $<.05$ \\
\hline 03/19/92 & -- & $<.05$ & .12 & $<.20$ & .05 & $<.05$ & .07 & $<.05$ & $<.05$ & $<.05$ & -- & $<.05$ \\
\hline 03/26/92 & -- & $<.05$ & .15 & $<.20$ & .05 & $<.05$ & .10 & $<.05$ & $<.05$ & $<.05$ & -- & .05 \\
\hline 03/30/92 & -- & $<.05$ & .19 & $<.20$ & .05 & $<.05$ & .08 & $<.05$ & $<.05$ & $<.05$ & -- & $<.05$ \\
\hline 04/10/92 & -- & $<.05$ & .18 & $<.20$ & $<.05$ & $<.05$ & .08 & $<.05$ & $<.05$ & $<.05$ & -- & $<.05$ \\
\hline $04 / 15 / 92$ & -- & $<.05$ & .22 & $<.20$ & .05 & $<.05$ & .08 & $<.05$ & $<.05$ & $<.05$ & -- & $<.05$ \\
\hline $04 / 23 / 92$ & -- & $<.05$ & .09 & $<.20$ & $<.05$ & $<.05$ & $<.10$ & $<.05$ & $<.05$ & $<.05$ & -- & $<.05$ \\
\hline 04/30/92 & -- & .09 & .64 & $<.20$ & $<.05$ & $<.05$ & .21 & $<.05$ & $<.05$ & $<.05$ & -- & $<.05$ \\
\hline 05/06/92 & -- & .10 & 1.1 & $<.20$ & .11 & $<.05$ & .52 & $<.05$ & $<.05$ & $<.05$ & -- & .09 \\
\hline 05/14/92 & -- & .08 & .64 & $<.20$ & .10 & $<.05$ & .41 & $<.05$ & $<.05$ & $<.05$ & -- & .07 \\
\hline $05 / 20 / 92$ & -- & $<.05$ & .31 & $<.20$ & .08 & $<.05$ & .16 & $<.05$ & $<.05$ & $<.05$ & -- & .08 \\
\hline $05 / 28 / 92$ & -- & $<.05$ & .32 & $<.20$ & .08 & $<.05$ & .14 & $<.05$ & $<.05$ & $<.05$ & -- & .09 \\
\hline 06/03/92 & -- & .06 & .37 & $<.20$ & .07 & $<.05$ & .17 & $<.05$ & $<.05$ & $<.05$ & -- & .08 \\
\hline 06/12/92 & -- & .16 & 1.1 & .80 & .14 & $<.10$ & .44 & .10 & $<.05$ & $<.05$ & -- & .14 \\
\hline 06/19/92 & -- & .09 & .89 & .30 & .15 & $<.10$ & .39 & .10 & $<.05$ & $<.05$ & -- & .14 \\
\hline $06 / 25 / 92$ & -- & .06 & .70 & .30 & .13 & .09 & .22 & .06 & $<.05$ & $<.05$ & -- & .09 \\
\hline 07/02/92 & -- & .05 & .76 & $<.20$ & .10 & .09 & .21 & .06 & $<.05$ & $<.05$ & -- & .08 \\
\hline 07/09/92 & -- & .05 & .81 & .30 & .16 & .10 & .19 & .05 & $<.05$ & $<.05$ & -- & .08 \\
\hline 07/16/92 & -- & .12 & 1.2 & .40 & .23 & .14 & .38 & .06 & $<.05$ & $<.05$ & -- & .10 \\
\hline
\end{tabular}


Table 2. Results of analysis of selected herbicides and degradation products by U.S. Geological Survey National Water-Quality Laboratory, Lakewood, Colorado, for water samples collected from the Mississippi River near St. Francisville and at Baton Rouge, 1991-2003.—Continued

[--, no data; <, less than; *, reported estimates from laboratory; ametryn, molinate, pendamethalin, propanil, propazine, and trifluralin were not detected]

\begin{tabular}{|c|c|c|c|c|c|c|c|c|c|c|c|c|}
\hline \multirow{2}{*}{$\begin{array}{c}\text { Date of } \\
\text { collec- } \\
\text { tion } \\
\text { (month/ } \\
\text { day/year) }\end{array}$} & \multicolumn{12}{|c|}{ Concentration, in micrograms per liter } \\
\hline & Acetochlor & Alachlor & Atrazine & $\begin{array}{c}\text { Cyana- } \\
\text { zine }\end{array}$ & $\begin{array}{l}\text { Deethyl- } \\
\text { atrazine }\end{array}$ & $\begin{array}{l}\text { Deiso- } \\
\text { propyl- } \\
\text { atrazine }\end{array}$ & $\begin{array}{l}\text { Metol- } \\
\text { achlor }\end{array}$ & Metribuzin & Prometon & Prometryn & Propachlor & Simazine \\
\hline \multicolumn{13}{|c|}{ Mississippi River at Baton Rouge, Louisiana, station 07374000 (fig. 1)—Continued } \\
\hline 07/23/92 & -- & 0.10 & 0.80 & 0.30 & 0.18 & 0.12 & 0.18 & $<0.05$ & $<0.05$ & $<0.05$ & -- & 0.09 \\
\hline $07 / 31 / 92$ & -- & .11 & 1.5 & .50 & .33 & .19 & .39 & .05 & $<.05$ & $<.05$ & -- & .10 \\
\hline 08/05/92 & -- & .10 & 1.4 & .40 & .36 & .22 & .47 & .05 & $<.05$ & $<.05$ & -- & .08 \\
\hline 08/13/92 & -- & .07 & 1.1 & .20 & .34 & .17 & .33 & .08 & .06 & $<.05$ & -- & .09 \\
\hline $08 / 21 / 92$ & -- & .07 & .85 & .20 & .32 & .16 & .25 & $<.05$ & .06 & $<.05$ & -- & $<.05$ \\
\hline 09/04/92 & -- & .05 & .50 & .20 & .23 & .12 & .16 & $<.05$ & .06 & $<.05$ & -- & $<.05$ \\
\hline 09/09/92 & -- & $<.05$ & .41 & $<.20$ & .21 & .12 & .11 & $<.05$ & .05 & $<.05$ & -- & $<.05$ \\
\hline 09/16/92 & -- & $<.05$ & .36 & $<.20$ & $<.05$ & $<.05$ & .08 & $<.05$ & $<.05$ & $<.05$ & -- & $<.05$ \\
\hline 09/23/92 & -- & $<.05$ & .33 & $<.20$ & $<.05$ & $<.05$ & .07 & $<.05$ & $<.05$ & $<.05$ & -- & $<.05$ \\
\hline
\end{tabular}


Table 3. Results of analysis of alachlor, atrazine, metolachlor, and simazine by the Jefferson Parish Water Quality Laboratory, Jefferson, Louisiana, for water samples collected from the Mississippi River at New Orleans, Louisiana, March through September 1993.

$[<$, less than]

\begin{tabular}{|c|c|c|c|c|}
\hline \multirow{2}{*}{$\begin{array}{l}\text { Date of collection } \\
\text { (month/day/year) }\end{array}$} & \multicolumn{4}{|c|}{ Concentration, in micrograms per liter } \\
\hline & Alachlor & Atrazine & Metolachlor & Simazine \\
\hline $03 / 03 / 93$ & $<0.03$ & 0.10 & 0.09 & 0.06 \\
\hline 03/10/93 & $<.03$ & .05 & .06 & .02 \\
\hline $03 / 17 / 93$ & $<.03$ & .06 & .03 & .02 \\
\hline $03 / 24 / 93$ & .05 & .02 & .06 & .07 \\
\hline $03 / 31 / 93$ & $<.03$ & .06 & .05 & .02 \\
\hline 04/07/93 & $<.01$ & .07 & .09 & .05 \\
\hline $04 / 14 / 93$ & $<.01$ & .07 & .07 & $<.01$ \\
\hline $04 / 21 / 93$ & $<.01$ & .08 & .09 & .01 \\
\hline $04 / 28 / 93$ & .03 & .20 & .16 & .12 \\
\hline $05 / 05 / 93$ & .03 & .35 & .17 & .18 \\
\hline $05 / 12 / 93$ & .03 & .37 & .18 & .31 \\
\hline $05 / 19 / 93$ & .11 & .85 & .10 & .14 \\
\hline $05 / 26 / 93$ & .06 & .90 & .27 & .35 \\
\hline 06/02/93 & .09 & 1.2 & .28 & .24 \\
\hline 06/09/93 & .05 & .70 & .32 & .09 \\
\hline 06/16/93 & .05 & 1.0 & .25 & .09 \\
\hline $06 / 23 / 93$ & .13 & 1.6 & .39 & .09 \\
\hline 06/30/93 & .40 & 4.2 & 1.3 & .18 \\
\hline $07 / 14 / 93$ & .36 & 3.8 & 1.5 & .13 \\
\hline $07 / 21 / 93$ & .20 & 3.1 & .87 & .20 \\
\hline $07 / 28 / 93$ & .27 & 3.1 & .65 & .21 \\
\hline 08/04/93 & .18 & 2.8 & .63 & .11 \\
\hline 08/11/93 & .12 & 1.2 & .63 & .15 \\
\hline 09/15/93 & .03 & 1.3 & .32 & .11 \\
\hline $09 / 22 / 93$ & .01 & .46 & .08 & .02 \\
\hline 09/29/93 & .01 & .66 & .07 & .02 \\
\hline
\end{tabular}


Table 4. Results of analysis of selected triazine herbicides and degradation products by gas chromatography/mass spectrometry methods GCS (1993-96) and GCR (1996-2003) at the U.S. Geological Survey Organic Geochemistry Research Laboratory, Lawrence, Kansas, for water samples collected from the Mississippi River at Baton Rouge, Louisiana, 1993-2003.

[R, regular sample; L, laboratory duplicate sample; --, no data; <, less than; ESA, ethanesulfonic acid; ELISA, enzyme-linked immunosorbent assay; ametryn, deisopropylprometryn, demethylnorflurazon, flufenacet, norflurazon, pendimethalin, propachlor, propanil, propazine, prometryn, terbutryn, and 3-trifluoromethylaniline were not detected]

\begin{tabular}{|c|c|c|c|c|c|c|c|c|c|c|c|c|c|c|c|c|c|c|c|c|}
\hline \multirow[b]{2}{*}{$\begin{array}{c}\text { Date of } \\
\text { collection } \\
\text { (month/ } \\
\text { day/year) }\end{array}$} & \multirow[b]{2}{*}{$\begin{array}{c}\text { Collec- } \\
\text { tion time } \\
(24- \\
\text { hour) }\end{array}$} & \multirow[b]{2}{*}{$\begin{array}{l}\text { Sam- } \\
\text { ple } \\
\text { type }\end{array}$} & \multicolumn{18}{|c|}{ Concentration, in micrograms per liter } \\
\hline & & & $\begin{array}{c}\text { Aceto- } \\
\text { chlor }\end{array}$ & $\begin{array}{l}\text { Ala- } \\
\text { chlor }\end{array}$ & $\begin{array}{c}\text { Ala- } \\
\text { chlor } \\
\text { ESA } \\
\text { ELISA }\end{array}$ & $\begin{array}{c}\text { Atra- } \\
\text { zine }\end{array}$ & $\begin{array}{l}\text { De- } \\
\text { ethyl- } \\
\text { atra- } \\
\text { zine }\end{array}$ & $\begin{array}{l}\text { Deiso- } \\
\text { propyl- } \\
\text { atra- } \\
\text { zine }\end{array}$ & $\begin{array}{l}\text { Cyan- } \\
\text { azine }\end{array}$ & $\begin{array}{l}\text { Cyan- } \\
\text { azine } \\
\text { amide }\end{array}$ & $\begin{array}{c}\text { De- } \\
\text { methyl- } \\
\text { fluo- } \\
\text { metu- } \\
\text { ron }\end{array}$ & $\begin{array}{c}\text { Di- } \\
\text { meth- } \\
\text { enamid }\end{array}$ & $\begin{array}{l}\text { Fluo- } \\
\text { met- } \\
\text { uron }\end{array}$ & $\begin{array}{l}\text { Meto- } \\
\text { lachlor }\end{array}$ & $\begin{array}{l}\text { Metri- } \\
\text { buzin }\end{array}$ & $\begin{array}{c}\text { Moli- } \\
\text { nate }\end{array}$ & $\begin{array}{l}\text { Pro- } \\
\text { meton }\end{array}$ & $\begin{array}{c}\text { Sima- } \\
\text { zine }\end{array}$ & $\begin{array}{c}\text { Tri- } \\
\text { fluralin }\end{array}$ & $\begin{array}{c}\text { 3-triflu- } \\
\text { oro- } \\
\text { methyl- } \\
\text { phenyl- } \\
\text { urea }\end{array}$ \\
\hline $07 / 07 / 93$ & 1100 & $\mathrm{R}$ & -- & 0.36 & 2.2 & 3.2 & 0.40 & 0.29 & 1.4 & 0.95 & -- & -- & -- & 1.0 & 0.10 & -- & 0.06 & 0.12 & -- & -- \\
\hline 07/09/93 & 1430 & $\mathrm{R}$ & -- & .37 & 2.0 & 3.3 & .46 & .31 & 1.5 & .99 & -- & -- & -- & 1.1 & .10 & -- & .07 & .09 & -- & -- \\
\hline $07 / 12 / 93$ & 1605 & $\mathrm{R}$ & -- & .32 & 2.8 & 3.0 & .52 & .31 & 1.2 & 1.1 & -- & -- & -- & 1.1 & .08 & -- & $<.05$ & .08 & -- & -- \\
\hline $07 / 15 / 93$ & 1440 & $\mathrm{R}$ & -- & .31 & 2.6 & 3.2 & .50 & .35 & 1.3 & .97 & -- & -- & -- & .97 & .08 & -- & .06 & .09 & -- & -- \\
\hline $07 / 19 / 93$ & 1333 & $\mathrm{R}$ & -- & .23 & 1.7 & 2.7 & .52 & .33 & 1.2 & .98 & -- & -- & -- & .87 & .13 & -- & $<.05$ & .07 & -- & -- \\
\hline $07 / 22 / 93$ & 0830 & $\mathrm{R}$ & -- & .17 & 1.4 & 2.1 & .43 & .27 & .84 & .78 & -- & -- & -- & .64 & .12 & -- & $<.05$ & .05 & -- & -- \\
\hline $07 / 26 / 93$ & 1400 & $\mathrm{R}$ & -- & .15 & 1.8 & 1.8 & .40 & .26 & .96 & -- & -- & -- & -- & .60 & $<.05$ & -- & .06 & $<.05$ & -- & -- \\
\hline $07 / 29 / 93$ & 1530 & $\mathrm{R}$ & -- & .14 & 2.0 & 1.7 & .38 & .27 & .75 & -- & -- & -- & -- & .56 & $<.05$ & -- & $<.05$ & $<.05$ & -- & -- \\
\hline $08 / 02 / 93$ & 1130 & $\mathrm{R}$ & -- & .12 & 1.7 & 1.6 & .33 & .22 & .73 & -- & -- & -- & -- & .52 & $<.05$ & -- & $<.05$ & $<.05$ & -- & -- \\
\hline $08 / 05 / 93$ & 1215 & $\mathrm{R}$ & -- & .13 & 2.3 & 2.0 & .45 & .35 & .74 & 1.2 & -- & -- & -- & .63 & $<.05$ & -- & $<.05$ & $<.05$ & -- & -- \\
\hline 08/06/93 & 1030 & $\mathrm{R}$ & -- & .14 & 1.2 & 2.2 & .51 & .38 & .76 & 1.2 & -- & -- & -- & .70 & $<.05$ & -- & $<.05$ & $<.05$ & -- & -- \\
\hline 08/09/93 & 1500 & $\mathrm{R}$ & -- & .10 & 1.7 & 1.8 & .46 & .37 & .64 & 1.0 & -- & -- & -- & .59 & $<.05$ & -- & $<.05$ & $<.05$ & -- & -- \\
\hline 08/09/93 & 1500 & $\mathrm{~L}$ & -- & .12 & 1.7 & 1.9 & .46 & .37 & .65 & -- & -- & -- & -- & .60 & $<.05$ & -- & $<.05$ & $<.05$ & -- & -- \\
\hline $08 / 12 / 93$ & 1200 & $\mathrm{R}$ & -- & .09 & 1.3 & 1.5 & .34 & .25 & .45 & .75 & -- & -- & -- & .53 & $<.05$ & -- & $<.05$ & $<.05$ & -- & -- \\
\hline $08 / 12 / 93$ & 1200 & $\mathrm{~L}$ & -- & .10 & -- & 1.8 & .40 & .28 & .53 & .75 & -- & -- & -- & .57 & $<.05$ & -- & $<.05$ & $<.05$ & -- & -- \\
\hline $08 / 16 / 93$ & 1440 & $\mathrm{R}$ & -- & .18 & 2.0 & 1.4 & .47 & .31 & .64 & .78 & -- & -- & -- & .54 & $<.05$ & -- & $<.05$ & $<.05$ & -- & -- \\
\hline $08 / 19 / 93$ & 1340 & $\mathrm{R}$ & -- & .15 & 2.2 & 1.4 & .46 & .31 & .58 & .80 & -- & -- & -- & .52 & $<.05$ & -- & $<.05$ & $<.05$ & -- & -- \\
\hline $08 / 23 / 93$ & 1300 & $\mathrm{R}$ & -- & .10 & 1.2 & 1.4 & .42 & .29 & .49 & .89 & -- & -- & -- & .48 & $<.05$ & -- & $<.05$ & $<.05$ & -- & -- \\
\hline $08 / 27 / 93$ & -- & $\mathrm{R}$ & -- & .09 & 1.3 & 1.4 & .42 & .28 & .51 & .78 & -- & -- & -- & .45 & $<.05$ & -- & $<.05$ & $<.05$ & -- & -- \\
\hline $08 / 30 / 93$ & 1040 & $\mathrm{R}$ & -- & .07 & 1.1 & 1.1 & .38 & .24 & .34 & .65 & -- & -- & -- & .35 & $<.05$ & -- & $<.05$ & $<.05$ & -- & -- \\
\hline
\end{tabular}


Table 4. Results of analysis of selected triazine herbicides and degradation products by gas chromatography/mass spectrometry methods GCS (1993-96) and GCR (1996-2003) at the U.S. Geological Survey Organic Geochemistry Research Laboratory, Lawrence, Kansas, for water samples collected from the Mississippi River at Baton Rouge,

Louisiana, 1993-2003.-Continued

[R, regular sample; L, laboratory duplicate sample; --, no data; <, less than; ESA, ethanesulfonic acid; ELISA, enzyme-linked immunosorbent assay; ametryn, deisopropylprometryn, demethylnorflurazon, flufenacet, norflurazon, pendimethalin, propachlor, propanil, propazine, prometryn, terbutryn, and 3-trifluoromethylaniline were not detected]

\begin{tabular}{|c|c|c|c|c|c|c|c|c|c|c|c|c|c|c|c|c|c|c|c|c|}
\hline \multirow[b]{2}{*}{$\begin{array}{l}\text { Date of } \\
\text { collection } \\
\text { (month/ } \\
\text { day/year) }\end{array}$} & \multirow[b]{2}{*}{$\begin{array}{l}\text { Collec- } \\
\text { tion } \\
\text { time } \\
(24- \\
\text { hour) }\end{array}$} & \multirow[b]{2}{*}{$\begin{array}{l}\text { Sam- } \\
\text { ple } \\
\text { type }\end{array}$} & \multicolumn{18}{|c|}{ Concentration, in micrograms per liter } \\
\hline & & & $\begin{array}{l}\text { Aceto- } \\
\text { chlor }\end{array}$ & $\begin{array}{l}\text { Ala- } \\
\text { chlor }\end{array}$ & $\begin{array}{l}\text { Ala- } \\
\text { chlor } \\
\text { ESA } \\
\text { ELISA }\end{array}$ & $\begin{array}{l}\text { Atra- } \\
\text { zine }\end{array}$ & $\begin{array}{l}\text { De- } \\
\text { ethyl- } \\
\text { atra- } \\
\text { zine }\end{array}$ & $\begin{array}{l}\text { Deiso- } \\
\text { propyl- } \\
\text { atrazine }\end{array}$ & $\begin{array}{l}\text { Cyan- } \\
\text { azine }\end{array}$ & $\begin{array}{l}\text { Cyan- } \\
\text { azine } \\
\text { amide }\end{array}$ & $\begin{array}{c}\text { De- } \\
\text { methyl- } \\
\text { fluo- } \\
\text { metu- } \\
\text { ron }\end{array}$ & $\begin{array}{c}\text { Di- } \\
\text { meth- } \\
\text { enamid }\end{array}$ & $\begin{array}{l}\text { Fluo- } \\
\text { met- } \\
\text { uron }\end{array}$ & $\begin{array}{l}\text { Meto- } \\
\text { lachlor }\end{array}$ & $\begin{array}{l}\text { Metri- } \\
\text { buzin }\end{array}$ & $\begin{array}{c}\text { Moli- } \\
\text { nate }\end{array}$ & $\begin{array}{l}\text { Pro- } \\
\text { meton }\end{array}$ & $\begin{array}{l}\text { Sima- } \\
\text { zine }\end{array}$ & $\begin{array}{c}\text { Tri- } \\
\text { fluralin }\end{array}$ & $\begin{array}{c}\text { 3-triflu- } \\
\text { oro- } \\
\text { methyl- } \\
\text { phenyl- } \\
\text { urea }\end{array}$ \\
\hline $09 / 02 / 93$ & 1100 & $\mathrm{R}$ & -- & 0.06 & 1.1 & 1.2 & 0.39 & 0.24 & 0.35 & 0.59 & -- & -- & -- & 0.32 & $<0.05$ & -- & $<0.05$ & $<0.05$ & -- & -- \\
\hline 09/07/93 & 1030 & $\mathrm{R}$ & -- & .05 & 1.2 & .93 & .32 & .19 & .24 & .32 & -- & -- & -- & .25 & $<.05$ & -- & $<.05$ & $<.05$ & -- & -- \\
\hline $09 / 16 / 93$ & -- & $\mathrm{R}$ & -- & .05 & 1.2 & .85 & .33 & .20 & .15 & .27 & -- & -- & -- & .25 & $<.05$ & -- & $<.05$ & $<.05$ & -- & -- \\
\hline 09/16/93 & -- & $\mathrm{L}$ & -- & .05 & .87 & .90 & .35 & .18 & .17 & .25 & -- & -- & -- & .25 & $<.05$ & -- & $<.05$ & $<.05$ & -- & -- \\
\hline $09 / 23 / 93$ & 1515 & $\mathrm{R}$ & -- & $<.05$ & 1.2 & .79 & .32 & .19 & .12 & .15 & -- & -- & -- & .21 & $<.05$ & -- & $<.05$ & $<.05$ & -- & -- \\
\hline $10 / 01 / 93$ & 1430 & $\mathrm{R}$ & -- & $<.05$ & 1.2 & 63 & .26 & .16 & .09 & .10 & -- & -- & -- & .15 & $<.05$ & -- & $<.05$ & $<.05$ & -- & -- \\
\hline $10 / 01 / 93$ & 1430 & $\mathrm{~L}$ & -- & $<.05$ & 1.2 & .64 & .24 & .14 & .08 & .10 & -- & -- & -- & .15 & $<.05$ & -- & $<.05$ & $<.05$ & -- & -- \\
\hline $10 / 08 / 93$ & 1600 & $\mathrm{R}$ & -- & $<.05$ & .73 & .46 & .17 & .10 & .06 & .08 & -- & -- & -- & .10 & $<.05$ & -- & $<.05$ & $<.05$ & -- & -- \\
\hline $10 / 15 / 93$ & 1500 & $\mathrm{R}$ & -- & $<.05$ & .89 & .46 & .18 & .10 & .06 & $<.05$ & -- & -- & -- & .09 & $<.05$ & -- & $<.05$ & $<.05$ & -- & -- \\
\hline $10 / 22 / 93$ & 1130 & $\mathrm{R}$ & -- & $<.05$ & .56 & .35 & .13 & .08 & .06 & $<.05$ & -- & -- & -- & .07 & $<.05$ & -- & $<.05$ & $<.05$ & -- & -- \\
\hline $10 / 22 / 93$ & 1130 & $\mathrm{~L}$ & -- & $<.05$ & 1.0 & .50 & .20 & .12 & .08 & $<.05$ & -- & -- & -- & .10 & $<.05$ & -- & $<.05$ & $<.05$ & -- & -- \\
\hline $11 / 05 / 93$ & 1230 & $\mathrm{R}$ & -- & $<.05$ & .78 & .42 & .15 & .09 & $<.05$ & .08 & -- & -- & -- & .11 & $<.05$ & -- & $<.05$ & $<.05$ & -- & -- \\
\hline $11 / 10 / 93$ & 1100 & $\mathrm{R}$ & -- & $<.05$ & .77 & .37 & .14 & .09 & .05 & .09 & -- & -- & -- & .10 & $<.05$ & -- & $<.05$ & $<.05$ & -- & -- \\
\hline $11 / 19 / 93$ & 1430 & $\mathrm{R}$ & -- & $<.05$ & 1.2 & .27 & .07 & .05 & $<.05$ & -- & -- & -- & -- & .07 & $<.05$ & -- & $<.05$ & $<.05$ & -- & -- \\
\hline $11 / 19 / 93$ & 1430 & $\mathrm{~L}$ & -- & $<.05$ & 1.1 & .35 & .09 & .07 & $<.05$ & -- & -- & -- & -- & .10 & $<.05$ & -- & $<.05$ & $<.05$ & -- & -- \\
\hline $11 / 24 / 93$ & 1715 & $\mathrm{R}$ & -- & $<.05$ & 1.1 & .31 & .08 & .06 & $<.05$ & -- & -- & -- & -- & .07 & $<.05$ & -- & $<.05$ & .06 & -- & -- \\
\hline $12 / 04 / 93$ & 1230 & $\mathrm{R}$ & -- & $<.05$ & .85 & .21 & $<.05$ & $<.05$ & $<.05$ & -- & -- & -- & -- & .07 & $<.05$ & -- & $<.05$ & $<.05$ & -- & -- \\
\hline $12 / 09 / 93$ & 1130 & $\mathrm{R}$ & -- & $<.05$ & .89 & .25 & .08 & $<.05$ & $<.05$ & -- & -- & -- & -- & .10 & $<.05$ & -- & $<.05$ & $<.05$ & -- & -- \\
\hline $12 / 22 / 93$ & 1400 & $\mathrm{R}$ & -- & $<.05$ & .68 & .17 & .05 & $<.05$ & $<.05$ & -- & -- & -- & -- & .07 & $<.05$ & -- & $<.05$ & $<.05$ & -- & -- \\
\hline $12 / 31 / 93$ & 1115 & $\mathrm{R}$ & -- & $<.05$ & .82 & .16 & $<.05$ & $<.05$ & $<.05$ & -- & -- & -- & -- & .05 & $<.05$ & -- & $<.05$ & $<.05$ & -- & -- \\
\hline
\end{tabular}


Table 4. Results of analysis of selected triazine herbicides and degradation products by gas chromatography/mass spectrometry methods GCS (1993-96) and GCR (1996-2003) at the U.S. Geological Survey Organic Geochemistry Research Laboratory, Lawrence, Kansas, for water samples collected from the Mississippi River at Baton Rouge,

Louisiana, 1993-2003.-Continued

[R, regular sample; L, laboratory duplicate sample; --, no data; <, less than; ESA, ethanesulfonic acid; ELISA, enzyme-linked immunosorbent assay; ametryn, deisopropylprometryn, demethylnorflurazon, flufenacet, norflurazon, pendimethalin, propachlor, propanil, propazine, prometryn, terbutryn, and 3-trifluoromethylaniline were not detected]

\begin{tabular}{|c|c|c|c|c|c|c|c|c|c|c|c|c|c|c|c|c|c|c|c|c|}
\hline \multirow[b]{2}{*}{$\begin{array}{l}\text { Date of } \\
\text { collection } \\
\text { (month/ } \\
\text { day/year) }\end{array}$} & \multirow[b]{2}{*}{$\begin{array}{l}\text { Collec- } \\
\text { tion } \\
\text { time } \\
\text { (24- } \\
\text { hour) }\end{array}$} & \multirow[b]{2}{*}{$\begin{array}{l}\text { Sam- } \\
\text { ple } \\
\text { type }\end{array}$} & \multicolumn{18}{|c|}{ Concentration, in micrograms per liter } \\
\hline & & & $\begin{array}{l}\text { Aceto- } \\
\text { chlor }\end{array}$ & $\begin{array}{l}\text { Ala- } \\
\text { chlor }\end{array}$ & $\begin{array}{l}\text { Ala- } \\
\text { chlor } \\
\text { ESA } \\
\text { ELISA }\end{array}$ & $\begin{array}{l}\text { Atra- } \\
\text { zine }\end{array}$ & $\begin{array}{l}\text { De- } \\
\text { ethyl- } \\
\text { atra- } \\
\text { zine }\end{array}$ & $\begin{array}{l}\text { Deiso- } \\
\text { propyl- } \\
\text { atrazine }\end{array}$ & $\begin{array}{l}\text { Cyan- } \\
\text { azine }\end{array}$ & $\begin{array}{l}\text { Cyan- } \\
\text { azine } \\
\text { amide }\end{array}$ & $\begin{array}{c}\text { De- } \\
\text { methyl- } \\
\text { fluo- } \\
\text { metu- } \\
\text { ron }\end{array}$ & $\begin{array}{c}\text { Di- } \\
\text { meth- } \\
\text { enamid }\end{array}$ & $\begin{array}{l}\text { Fluo- } \\
\text { met- } \\
\text { uron }\end{array}$ & $\begin{array}{l}\text { Meto- } \\
\text { lachlor }\end{array}$ & $\begin{array}{c}\text { Metri- } \\
\text { buzin }\end{array}$ & $\begin{array}{c}\text { Moli- } \\
\text { nate }\end{array}$ & $\begin{array}{l}\text { Pro- } \\
\text { meton }\end{array}$ & $\begin{array}{c}\text { Sima- } \\
\text { zine }\end{array}$ & $\begin{array}{c}\text { Tri- } \\
\text { fluralin }\end{array}$ & $\begin{array}{c}\text { 3-triflu- } \\
\text { oro- } \\
\text { methyl- } \\
\text { phenyl- } \\
\text { urea }\end{array}$ \\
\hline $01 / 06 / 94$ & 1415 & $\mathrm{R}$ & -- & $<0.05$ & 0.83 & 0.16 & $<0.05$ & $<0.05$ & $<0.05$ & -- & -- & -- & -- & 0.06 & $<0.05$ & -- & $<0.05$ & $<0.05$ & -- & -- \\
\hline $01 / 13 / 94$ & 1430 & $\mathrm{R}$ & -- & $<.05$ & .67 & .17 & $<.05$ & $<.05$ & $<.05$ & -- & -- & -- & -- & .05 & $<.05$ & -- & $<.05$ & $<.05$ & -- & -- \\
\hline $01 / 21 / 94$ & 1615 & $\mathrm{R}$ & -- & $<.05$ & .37 & .09 & $<.05$ & $<.05$ & $<.05$ & -- & -- & -- & -- & $<.05$ & $<.05$ & -- & $<.05$ & $<.05$ & -- & -- \\
\hline $01 / 21 / 94$ & 1615 & $\mathrm{~L}$ & -- & $<.05$ & .39 & .10 & $<.05$ & $<.05$ & $<.05$ & -- & -- & -- & -- & $<.05$ & $<.05$ & -- & $<.05$ & $<.05$ & -- & -- \\
\hline $01 / 27 / 94$ & 1630 & $\mathrm{R}$ & -- & $<.05$ & .25 & .08 & $<.05$ & $<.05$ & $<.05$ & -- & -- & -- & -- & $<.05$ & $<.05$ & -- & $<.05$ & $<.05$ & -- & -- \\
\hline 02/04/94 & 1145 & $\mathrm{R}$ & -- & $<.05$ & .28 & .07 & $<.05$ & $<.05$ & $<.05$ & -- & -- & -- & -- & $<.05$ & $<.05$ & -- & $<.05$ & $<.05$ & -- & -- \\
\hline $02 / 10 / 94$ & 1200 & $\mathrm{R}$ & -- & $<.05$ & .30 & .09 & $<.05$ & $<.05$ & $<.05$ & -- & -- & -- & -- & $<.05$ & $<.05$ & -- & $<.05$ & $<.05$ & -- & -- \\
\hline $02 / 17 / 94$ & 1400 & $\mathrm{R}$ & -- & $<.05$ & .39 & .09 & $<.05$ & $<.05$ & $<.05$ & -- & -- & -- & -- & $<.05$ & $<.05$ & -- & $<.05$ & $<.05$ & -- & -- \\
\hline $02 / 25 / 94$ & 1500 & $\mathrm{R}$ & -- & $<.05$ & .27 & .05 & $<.05$ & $<.05$ & $<.05$ & -- & -- & -- & -- & $<.05$ & $<.05$ & -- & $<.05$ & $<.05$ & -- & -- \\
\hline $03 / 04 / 94$ & 1700 & $\mathrm{R}$ & -- & $<.05$ & .33 & .08 & $<.05$ & $<.05$ & $<.05$ & -- & -- & -- & -- & $<.05$ & $<.05$ & -- & $<.05$ & $<.05$ & -- & -- \\
\hline $03 / 04 / 94$ & 1700 & $\mathrm{~L}$ & -- & $<.05$ & -- & .06 & $<.05$ & $<.05$ & $<.05$ & -- & -- & -- & -- & $<.05$ & $<.05$ & -- & $<.05$ & $<.05$ & -- & -- \\
\hline $03 / 11 / 94$ & 1700 & $\mathrm{R}$ & -- & $<.05$ & .40 & .08 & $<.05$ & $<.05$ & $<.05$ & -- & -- & -- & -- & $<.05$ & $<.05$ & -- & $<.05$ & $<.05$ & -- & -- \\
\hline $03 / 23 / 94$ & 1330 & $\mathrm{R}$ & -- & $<.05$ & .35 & .09 & $<.05$ & $<.05$ & $<.05$ & -- & -- & -- & -- & $<.05$ & $<.05$ & -- & $<.05$ & $<.05$ & -- & -- \\
\hline $04 / 01 / 94$ & 1615 & $\mathrm{R}$ & -- & $<.05$ & $<.10$ & .11 & $<.05$ & $<.05$ & $<.05$ & -- & -- & -- & -- & $<.05$ & $<.05$ & -- & $<.05$ & $<.05$ & -- & -- \\
\hline 04/08/94 & 1645 & $\mathrm{R}$ & -- & $<.05$ & .34 & .33 & $<.05$ & $<.05$ & $<.05$ & -- & -- & -- & -- & .13 & $<.05$ & -- & $<.05$ & $<.05$ & -- & -- \\
\hline $04 / 22 / 94$ & 1315 & $\mathrm{R}$ & $<0.05$ & $<.05$ & .39 & .38 & $<.05$ & $<.05$ & $<.05$ & -- & -- & -- & -- & .13 & $<.05$ & -- & $<.05$ & $<.05$ & -- & -- \\
\hline $04 / 28 / 94$ & 1245 & $\mathrm{R}$ & $<.05$ & .07 & .41 & .77 & $<.05$ & $<.05$ & .27 & -- & -- & -- & -- & .30 & $<.05$ & -- & $<.05$ & .08 & -- & -- \\
\hline $05 / 04 / 94$ & 1630 & $\mathrm{R}$ & $<.05$ & $<.05$ & $<.10$ & .41 & .11 & $<.05$ & $<.05$ & -- & -- & -- & -- & $<.05$ & $<.05$ & -- & $<.05$ & .06 & -- & -- \\
\hline $05 / 13 / 94$ & 1530 & $\mathrm{R}$ & $<.05$ & .20 & .56 & 2.1 & .13 & .09 & .93 & -- & -- & -- & -- & .84 & $<.05$ & -- & $<.05$ & .19 & -- & -- \\
\hline $06 / 24 / 94$ & 1050 & $\mathrm{R}$ & $<.05$ & .15 & .82 & 2.0 & .24 & .18 & 1.1 & -- & -- & -- & -- & .71 & $<.05$ & -- & $<.05$ & .08 & -- & -- \\
\hline
\end{tabular}


Table 4. Results of analysis of selected triazine herbicides and degradation products by gas chromatography/mass spectrometry methods GCS (1993-96) and GCR (1996-2003) at the U.S. Geological Survey Organic Geochemistry Research Laboratory, Lawrence, Kansas, for water samples collected from the Mississippi River at Baton Rouge,

Louisiana, 1993-2003.-Continued

[R, regular sample; L, laboratory duplicate sample; --, no data; <, less than; ESA, ethanesulfonic acid; ELISA, enzyme-linked immunosorbent assay; ametryn, deisopropylprometryn, demethylnorflurazon, flufenacet, norflurazon, pendimethalin, propachlor, propanil, propazine, prometryn, terbutryn, and 3-trifluoromethylaniline were not detected]

\begin{tabular}{|c|c|c|c|c|c|c|c|c|c|c|c|c|c|c|c|c|c|c|c|c|}
\hline \multirow[b]{2}{*}{$\begin{array}{l}\text { Date of } \\
\text { collection } \\
\text { (month/ } \\
\text { day/year) }\end{array}$} & \multirow[b]{2}{*}{$\begin{array}{l}\text { Collec- } \\
\text { tion } \\
\text { time } \\
\text { (24- } \\
\text { hour) }\end{array}$} & \multirow[b]{2}{*}{$\begin{array}{l}\text { Sam- } \\
\text { ple } \\
\text { type }\end{array}$} & \multicolumn{18}{|c|}{ Concentration, in micrograms per liter } \\
\hline & & & $\begin{array}{l}\text { Aceto- } \\
\text { chlor }\end{array}$ & $\begin{array}{l}\text { Ala- } \\
\text { chlor }\end{array}$ & $\begin{array}{l}\text { Ala- } \\
\text { chlor } \\
\text { ESA } \\
\text { ELISA }\end{array}$ & $\begin{array}{c}\text { Atra- } \\
\text { zine }\end{array}$ & $\begin{array}{l}\text { De- } \\
\text { ethyl- } \\
\text { atra- } \\
\text { zine }\end{array}$ & $\begin{array}{l}\text { Deiso- } \\
\text { propyl- } \\
\text { atrazine }\end{array}$ & $\begin{array}{l}\text { Cyan- } \\
\text { azine }\end{array}$ & $\begin{array}{l}\text { Cyan- } \\
\text { azine } \\
\text { amide }\end{array}$ & $\begin{array}{c}\text { De- } \\
\text { methyl- } \\
\text { fluo- } \\
\text { metu- } \\
\text { ron }\end{array}$ & $\begin{array}{c}\text { Di- } \\
\text { meth- } \\
\text { enamid }\end{array}$ & $\begin{array}{l}\text { Fluo- } \\
\text { met- } \\
\text { uron }\end{array}$ & $\begin{array}{l}\text { Meto- } \\
\text { lachlor }\end{array}$ & $\begin{array}{l}\text { Metri- } \\
\text { buzin }\end{array}$ & $\begin{array}{l}\text { Moli- } \\
\text { nate }\end{array}$ & $\begin{array}{l}\text { Pro- } \\
\text { meton }\end{array}$ & $\begin{array}{l}\text { Sima- } \\
\text { zine }\end{array}$ & $\begin{array}{c}\text { Tri- } \\
\text { fluralin }\end{array}$ & $\begin{array}{c}\text { 3-triflu- } \\
\text { oro- } \\
\text { methyl- } \\
\text { phenyl- } \\
\text { urea }\end{array}$ \\
\hline $06 / 24 / 94$ & 1050 & $\mathrm{~L}$ & $<0.05$ & 0.14 & 0.90 & 2.0 & 0.24 & 0.18 & 0.96 & -- & -- & -- & -- & 0.70 & $<0.05$ & -- & $<0.05$ & 0.06 & -- & -- \\
\hline 07/01/94 & 1645 & $\mathrm{R}$ & $<.05$ & .12 & .96 & 2.0 & .25 & .18 & .87 & -- & -- & -- & -- & .65 & $<.05$ & -- & $<.05$ & .07 & -- & -- \\
\hline 07/07/94 & 1500 & $\mathrm{R}$ & $<.05$ & .11 & 1.2 & 2.1 & .29 & .24 & 1.1 & -- & -- & -- & -- & .81 & $<.05$ & -- & $<.05$ & .06 & -- & -- \\
\hline $07 / 15 / 94$ & 1210 & $\mathrm{R}$ & $<.05$ & .07 & 1.0 & 1.6 & .28 & .23 & .77 & -- & -- & -- & -- & .56 & $<.05$ & -- & $<.05$ & .06 & -- & -- \\
\hline $07 / 22 / 94$ & 1200 & $\mathrm{R}$ & $<.05$ & $<.05$ & .76 & 1.1 & .22 & .17 & .60 & -- & -- & -- & -- & .50 & $<.05$ & -- & $<.05$ & $<.05$ & -- & -- \\
\hline 08/05/94 & 1300 & $\mathrm{R}$ & $<.05$ & $<.05$ & .97 & .67 & .16 & .12 & .36 & -- & -- & -- & -- & .22 & $<.05$ & -- & $<.05$ & $<.05$ & -- & -- \\
\hline $08 / 05 / 94$ & 1300 & $\mathrm{~L}$ & $<.05$ & $<.05$ & 1.1 & .66 & .16 & .11 & .38 & -- & -- & -- & -- & .22 & $<.05$ & -- & $<.05$ & $<.05$ & -- & -- \\
\hline 08/09/94 & 1100 & $\mathrm{R}$ & $<.05$ & $<.05$ & 1.1 & .68 & .16 & .09 & .31 & -- & -- & -- & -- & .20 & $<.05$ & -- & $<.05$ & $<.05$ & -- & -- \\
\hline 08/19/94 & 1300 & $\mathrm{R}$ & $<.05$ & $<.05$ & .66 & .35 & .10 & .06 & .30 & -- & -- & -- & -- & .09 & $<.05$ & -- & $<.05$ & $<.05$ & -- & -- \\
\hline 08/29/94 & 1335 & $\mathrm{R}$ & $<.05$ & $<.05$ & .54 & .23 & .07 & .05 & .05 & -- & -- & -- & -- & .06 & $<.05$ & -- & $<.05$ & $<.05$ & -- & -- \\
\hline 08/29/94 & 1335 & $\mathrm{~L}$ & $<.05$ & $<.05$ & .55 & .24 & .07 & .05 & .05 & -- & -- & -- & -- & .07 & $<.05$ & -- & $<.05$ & $<.05$ & -- & -- \\
\hline 09/08/94 & 1445 & $\mathrm{R}$ & -- & -- & .50 & -- & -- & -- & -- & -- & -- & -- & -- & -- & -- & -- & -- & -- & -- & -- \\
\hline 09/16/94 & 1430 & $\mathrm{R}$ & $<.05$ & $<.05$ & .51 & .28 & .07 & .05 & .05 & -- & -- & -- & -- & .11 & $<.05$ & -- & $<.05$ & $<.05$ & -- & -- \\
\hline $09 / 23 / 94$ & 1515 & $\mathrm{R}$ & $<.05$ & $<.05$ & .47 & .28 & .07 & .07 & .06 & -- & -- & -- & -- & .11 & $<.05$ & -- & $<.05$ & $<.05$ & -- & -- \\
\hline 09/30/94 & 1415 & $\mathrm{R}$ & $<.05$ & $<.05$ & .43 & .19 & .05 & $<.05$ & $<.05$ & -- & -- & -- & -- & .05 & $<.05$ & -- & $<.05$ & $<.05$ & -- & -- \\
\hline $10 / 05 / 94$ & 1500 & $\mathrm{R}$ & $<.05$ & $<.05$ & -- & .22 & .06 & $<.05$ & $<.05$ & -- & -- & -- & -- & .05 & $<.05$ & -- & $<.05$ & $<.05$ & -- & -- \\
\hline $10 / 13 / 94$ & 1345 & $\mathrm{R}$ & $<.05$ & $<.05$ & .51 & .18 & .07 & $<.05$ & $<.05$ & -- & -- & -- & -- & $<.05$ & $<.05$ & -- & $<.05$ & $<.05$ & -- & -- \\
\hline $10 / 13 / 94$ & 1345 & $\mathrm{~L}$ & $<.05$ & $<.05$ & .58 & .18 & .07 & $<.05$ & $<.05$ & -- & -- & -- & -- & $<.05$ & $<.05$ & -- & $<.05$ & $<.05$ & -- & -- \\
\hline $10 / 21 / 94$ & 0915 & $\mathrm{R}$ & $<.05$ & $<.05$ & .46 & .16 & .05 & $<.05$ & $<.05$ & -- & -- & -- & -- & .05 & $<.05$ & -- & $<.05$ & $<.05$ & -- & -- \\
\hline $10 / 26 / 94$ & 1530 & $\mathrm{R}$ & $<.05$ & $<.05$ & .46 & .16 & .05 & $<.05$ & $<.05$ & -- & -- & -- & -- & .05 & $<.05$ & -- & $<.05$ & $<.05$ & -- & -- \\
\hline
\end{tabular}


Table 4. Results of analysis of selected triazine herbicides and degradation products by gas chromatography/mass spectrometry methods GCS (1993-96) and GCR (1996-2003) at the U.S. Geological Survey Organic Geochemistry Research Laboratory, Lawrence, Kansas, for water samples collected from the Mississippi River at Baton Rouge,

Louisiana, 1993-2003.-Continued

[R, regular sample; L, laboratory duplicate sample; --, no data; <, less than; ESA, ethanesulfonic acid; ELISA, enzyme-linked immunosorbent assay; ametryn, deisopropylprometryn, demethylnorflurazon, flufenacet, norflurazon, pendimethalin, propachlor, propanil, propazine, prometryn, terbutryn, and 3-trifluoromethylaniline were not detected]

\begin{tabular}{|c|c|c|c|c|c|c|c|c|c|c|c|c|c|c|c|c|c|c|c|c|}
\hline \multirow[b]{2}{*}{$\begin{array}{l}\text { Date of } \\
\text { collection } \\
\text { (month/ } \\
\text { day/year) }\end{array}$} & \multirow[b]{2}{*}{$\begin{array}{l}\text { Collec- } \\
\text { tion } \\
\text { time } \\
(24- \\
\text { hour) }\end{array}$} & \multirow[b]{2}{*}{$\begin{array}{l}\text { Sam- } \\
\text { ple } \\
\text { type }\end{array}$} & \multicolumn{18}{|c|}{ Concentration, in micrograms per liter } \\
\hline & & & $\begin{array}{l}\text { Aceto- } \\
\text { chlor }\end{array}$ & $\begin{array}{l}\text { Ala- } \\
\text { chlor }\end{array}$ & $\begin{array}{l}\text { Ala- } \\
\text { chlor } \\
\text { ESA } \\
\text { ELISA }\end{array}$ & $\begin{array}{l}\text { Atra- } \\
\text { zine }\end{array}$ & $\begin{array}{l}\text { De- } \\
\text { ethyl- } \\
\text { atra- } \\
\text { zine }\end{array}$ & $\begin{array}{l}\text { Deiso- } \\
\text { propyl- } \\
\text { atrazine }\end{array}$ & $\begin{array}{l}\text { Cyan- } \\
\text { azine }\end{array}$ & $\begin{array}{l}\text { Cyan- } \\
\text { azine } \\
\text { amide }\end{array}$ & $\begin{array}{c}\text { De- } \\
\text { methyl- } \\
\text { fluo- } \\
\text { metu- } \\
\text { ron }\end{array}$ & $\begin{array}{c}\text { Di- } \\
\text { meth- } \\
\text { enamid }\end{array}$ & $\begin{array}{l}\text { Fluo- } \\
\text { met- } \\
\text { uron }\end{array}$ & $\begin{array}{l}\text { Meto- } \\
\text { lachlor }\end{array}$ & $\begin{array}{l}\text { Metri- } \\
\text { buzin }\end{array}$ & $\begin{array}{l}\text { Moli- } \\
\text { nate }\end{array}$ & $\begin{array}{l}\text { Pro- } \\
\text { meton }\end{array}$ & $\begin{array}{l}\text { Sima- } \\
\text { zine }\end{array}$ & $\begin{array}{c}\text { Tri- } \\
\text { fluralin }\end{array}$ & $\begin{array}{c}\text { 3-triflu- } \\
\text { oro- } \\
\text { methyl- } \\
\text { phenyl- } \\
\text { urea }\end{array}$ \\
\hline $11 / 04 / 94$ & 1230 & $\mathrm{R}$ & $<0.05$ & $<0.05$ & 0.40 & 0.15 & 0.05 & $<0.05$ & $<0.05$ & -- & -- & -- & -- & 0.07 & $<0.05$ & -- & $<0.05$ & $<0.05$ & -- & -- \\
\hline $11 / 10 / 94$ & 1205 & $\mathrm{R}$ & $<.05$ & $<.05$ & .49 & .13 & .05 & $<.05$ & $<.05$ & -- & -- & -- & -- & $<.05$ & $<.05$ & -- & $<.05$ & $<.05$ & -- & -- \\
\hline $11 / 10 / 94$ & 1205 & $\mathrm{~L}$ & $<.05$ & $<.05$ & .53 & .15 & .06 & $<.05$ & $<.05$ & -- & -- & -- & -- & $<.05$ & $<.05$ & -- & $<.05$ & $<.05$ & -- & -- \\
\hline $11 / 10 / 94$ & 1315 & $\mathrm{R}$ & $<.05$ & $<.05$ & .52 & .18 & .05 & $<.05$ & $<.05$ & -- & -- & -- & -- & $<.05$ & $<.05$ & -- & $<.05$ & $<.05$ & -- & -- \\
\hline $11 / 23 / 94$ & 1400 & $\mathrm{R}$ & $<.05$ & $<.05$ & .41 & .31 & .05 & $<.05$ & $<.05$ & -- & -- & -- & -- & .07 & $<.05$ & -- & $<.05$ & $<.05$ & -- & -- \\
\hline $12 / 01 / 94$ & 1420 & $\mathrm{R}$ & $<.05$ & $<.05$ & .41 & $<.05$ & .05 & $<.05$ & $<.05$ & -- & -- & -- & -- & .05 & $<.05$ & -- & $<.05$ & $<.05$ & -- & -- \\
\hline $12 / 07 / 94$ & 1415 & $\mathrm{R}$ & $<.05$ & $<.05$ & .35 & .24 & .06 & $<.05$ & .06 & -- & -- & -- & -- & .06 & $<.05$ & -- & $<.05$ & $<.05$ & -- & -- \\
\hline $12 / 07 / 94$ & 1415 & $\mathrm{~L}$ & $<.05$ & $<.05$ & .25 & .24 & .06 & $<.05$ & .06 & -- & -- & -- & -- & .06 & $<.05$ & -- & $<.05$ & $<.05$ & -- & -- \\
\hline $12 / 15 / 94$ & 1300 & $\mathrm{R}$ & $<.05$ & $<.05$ & .31 & .24 & .06 & $<.05$ & $<.05$ & -- & -- & -- & -- & .06 & $<.05$ & -- & $<.05$ & $<.05$ & -- & -- \\
\hline $12 / 23 / 94$ & -- & $\mathrm{R}$ & $<.05$ & $<.05$ & .32 & .19 & .05 & $<.05$ & $<.05$ & -- & -- & -- & -- & .06 & $<.05$ & -- & $<.05$ & $<.05$ & -- & -- \\
\hline $01 / 05 / 95$ & 1250 & $\mathrm{R}$ & $<.05$ & $<.05$ & .36 & .17 & .05 & $<.05$ & .07 & -- & -- & -- & -- & .05 & $<.05$ & -- & $<.05$ & $<.05$ & -- & -- \\
\hline $01 / 13 / 95$ & 1140 & $\mathrm{R}$ & $<.05$ & $<.05$ & .41 & .14 & $<.05$ & $<.05$ & $<.05$ & -- & -- & -- & -- & .05 & $<.05$ & -- & $<.05$ & $<.05$ & -- & -- \\
\hline $01 / 19 / 95$ & 1430 & $\mathrm{R}$ & $<.05$ & $<.05$ & .23 & .13 & .05 & $<.05$ & $<.05$ & -- & -- & -- & -- & $<.05$ & $<.05$ & -- & $<.05$ & .05 & -- & -- \\
\hline $01 / 27 / 95$ & 1530 & $\mathrm{R}$ & $<.05$ & $<.05$ & .31 & .12 & $<.05$ & $<.05$ & $<.05$ & -- & -- & -- & -- & $<.05$ & $<.05$ & -- & $<.05$ & $<.05$ & -- & -- \\
\hline $02 / 03 / 95$ & 1530 & $\mathrm{R}$ & $<.05$ & $<.05$ & .34 & .14 & .05 & $<.05$ & $<.05$ & -- & -- & -- & -- & $<.05$ & $<.05$ & -- & $<.05$ & $<.05$ & -- & -- \\
\hline $02 / 03 / 95$ & 1530 & $\mathrm{~L}$ & $<.05$ & $<.05$ & .34 & .14 & $<.05$ & $<.05$ & $<.05$ & -- & -- & -- & -- & .06 & $<.05$ & -- & $<.05$ & $<.05$ & -- & -- \\
\hline $02 / 10 / 95$ & 1600 & $\mathrm{R}$ & $<.05$ & $<.05$ & .39 & .17 & .05 & $<.05$ & $<.05$ & -- & -- & -- & -- & .06 & $<.05$ & -- & $<.05$ & $<.05$ & -- & -- \\
\hline $02 / 17 / 95$ & 1530 & $\mathrm{R}$ & $<.05$ & $<.05$ & .30 & .17 & .05 & $<.05$ & $<.05$ & -- & -- & -- & -- & .05 & $<.05$ & -- & $<.05$ & $<.05$ & -- & -- \\
\hline $02 / 24 / 95$ & 1400 & $\mathrm{R}$ & $<.05$ & $<.05$ & .31 & .15 & .05 & $<.05$ & $<.05$ & -- & -- & -- & -- & $<.05$ & $<.05$ & -- & $<.05$ & $<.05$ & -- & -- \\
\hline $03 / 02 / 95$ & 1015 & $\mathrm{R}$ & $<.05$ & $<.05$ & .20 & .10 & $<.05$ & $<.05$ & $<.05$ & -- & -- & -- & -- & $<.05$ & $<.05$ & -- & $<.05$ & $<.05$ & -- & -- \\
\hline
\end{tabular}


Table 4. Results of analysis of selected triazine herbicides and degradation products by gas chromatography/mass spectrometry methods GCS (1993-96) and GCR (1996-2003) at the U.S. Geological Survey Organic Geochemistry Research Laboratory, Lawrence, Kansas, for water samples collected from the Mississippi River at Baton Rouge,

Louisiana, 1993-2003.-Continued

[R, regular sample; L, laboratory duplicate sample; --, no data; <, less than; ESA, ethanesulfonic acid; ELISA, enzyme-linked immunosorbent assay; ametryn, deisopropylprometryn, demethylnorflurazon, flufenacet, norflurazon, pendimethalin, propachlor, propanil, propazine, prometryn, terbutryn, and 3-trifluoromethylaniline were not detected]

\begin{tabular}{|c|c|c|c|c|c|c|c|c|c|c|c|c|c|c|c|c|c|c|c|c|}
\hline \multirow[b]{2}{*}{$\begin{array}{c}\text { Date of } \\
\text { collection } \\
\text { (month/ } \\
\text { day/year) }\end{array}$} & \multirow[b]{2}{*}{$\begin{array}{l}\text { Collec- } \\
\text { tion } \\
\text { time } \\
\text { (24- } \\
\text { hour) }\end{array}$} & \multirow[b]{2}{*}{$\begin{array}{l}\text { Sam- } \\
\text { ple } \\
\text { type }\end{array}$} & \multicolumn{18}{|c|}{ Concentration, in micrograms per liter } \\
\hline & & & $\begin{array}{l}\text { Aceto- } \\
\text { chlor }\end{array}$ & $\begin{array}{l}\text { Ala- } \\
\text { chlor }\end{array}$ & $\begin{array}{l}\text { Ala- } \\
\text { chlor } \\
\text { ESA } \\
\text { ELISA }\end{array}$ & $\begin{array}{l}\text { Atra- } \\
\text { zine }\end{array}$ & $\begin{array}{l}\text { De- } \\
\text { ethyl- } \\
\text { atra- } \\
\text { zine }\end{array}$ & $\begin{array}{l}\text { Deiso- } \\
\text { propyl- } \\
\text { atrazine }\end{array}$ & $\begin{array}{l}\text { Cyan- } \\
\text { azine }\end{array}$ & $\begin{array}{l}\text { Cyan- } \\
\text { azine } \\
\text { amide }\end{array}$ & $\begin{array}{c}\text { De- } \\
\text { methyl- } \\
\text { fluo- } \\
\text { metu- } \\
\text { ron }\end{array}$ & $\begin{array}{l}\text { Di- } \\
\text { meth- } \\
\text { enamid }\end{array}$ & $\begin{array}{l}\text { Fluo- } \\
\text { met- } \\
\text { uron }\end{array}$ & $\begin{array}{l}\text { Meto- } \\
\text { lachlor }\end{array}$ & $\begin{array}{c}\text { Metri- } \\
\text { buzin }\end{array}$ & $\begin{array}{l}\text { Moli- } \\
\text { nate }\end{array}$ & $\begin{array}{l}\text { Pro- } \\
\text { meton }\end{array}$ & $\begin{array}{c}\text { Sima- } \\
\text { zine }\end{array}$ & $\begin{array}{c}\text { Tri- } \\
\text { fluralin }\end{array}$ & $\begin{array}{l}\text { 3-triflu- } \\
\text { oro- } \\
\text { methyl- } \\
\text { phenyl- } \\
\text { urea }\end{array}$ \\
\hline $03 / 02 / 95$ & 1015 & $\mathrm{~L}$ & $<0.05$ & $<0.05$ & 0.24 & 0.10 & $<0.05$ & $<0.05$ & $<0.05$ & -- & -- & -- & -- & $<0.05$ & $<0.05$ & -- & $<0.05$ & $<0.05$ & -- & -- \\
\hline $03 / 10 / 95$ & 1410 & $\mathrm{R}$ & $<.05$ & $<.05$ & .26 & .10 & $<.05$ & $<.05$ & $<.05$ & -- & -- & -- & -- & $<.05$ & $<.05$ & -- & $<.05$ & $<.05$ & -- & -- \\
\hline $03 / 15 / 95$ & 1515 & $\mathrm{R}$ & $<.05$ & $<.05$ & .32 & .13 & $<.05$ & $<.05$ & $<.05$ & -- & -- & -- & -- & .07 & $<.05$ & -- & $<.05$ & $<.05$ & -- & -- \\
\hline $03 / 29 / 95$ & 1530 & $\mathrm{R}$ & .07 & $<.05$ & .47 & .20 & $<.05$ & $<.05$ & $<.05$ & $<0.05$ & -- & -- & -- & .09 & $<.05$ & -- & $<.05$ & .06 & -- & -- \\
\hline $04 / 05 / 95$ & 1510 & $\mathrm{R}$ & $<.05$ & $<.05$ & .54 & .24 & .05 & $<.05$ & .09 & $<.05$ & -- & -- & -- & .11 & $<.05$ & -- & $<.05$ & $<.05$ & -- & -- \\
\hline $04 / 14 / 95$ & 1315 & $\mathrm{R}$ & $<.05$ & $<.05$ & .59 & .22 & $<.05$ & $<.05$ & $<.05$ & $<.05$ & -- & -- & -- & .10 & $<.05$ & -- & $<.05$ & $<.05$ & -- & -- \\
\hline $04 / 21 / 95$ & 1530 & $\mathrm{R}$ & $<.05$ & $<.05$ & .75 & .44 & $<.05$ & $<.05$ & $<.05$ & $<.05$ & -- & -- & -- & .27 & $<.05$ & -- & $<.05$ & $<.05$ & -- & -- \\
\hline $04 / 21 / 95$ & 1530 & $\mathrm{~L}$ & $<.05$ & $<.05$ & .84 & .46 & $<.05$ & $<.05$ & .09 & $<.05$ & -- & -- & -- & .28 & $<.05$ & -- & $<.05$ & $<.05$ & -- & -- \\
\hline $04 / 28 / 95$ & 1115 & $\mathrm{R}$ & .06 & $<.05$ & .61 & .65 & .08 & $<.05$ & .24 & .07 & -- & -- & -- & .49 & .07 & -- & $<.05$ & .05 & -- & -- \\
\hline $05 / 05 / 95$ & 1515 & $\mathrm{R}$ & .08 & $<.05$ & .81 & 1.1 & .13 & .06 & .16 & .07 & -- & -- & -- & .38 & $<.05$ & -- & $<.05$ & .14 & -- & -- \\
\hline $05 / 15 / 95$ & 1430 & $\mathrm{R}$ & .09 & $<.05$ & .67 & .95 & .12 & .53 & .15 & .10 & -- & -- & -- & .30 & $<.05$ & -- & $<.05$ & .13 & -- & -- \\
\hline $05 / 19 / 95$ & 1330 & $\mathrm{R}$ & .09 & $<.05$ & .67 & 1.1 & .13 & .14 & .37 & .18 & -- & -- & -- & .27 & $<.05$ & -- & $<.05$ & .07 & -- & -- \\
\hline $05 / 19 / 95$ & 1330 & $\mathrm{~L}$ & .09 & $<.05$ & .68 & 1.1 & .14 & .24 & .43 & .23 & -- & -- & -- & .28 & $<.05$ & -- & $<.05$ & .08 & -- & -- \\
\hline $05 / 26 / 95$ & 1400 & $\mathrm{R}$ & .19 & $<.05$ & .63 & 1.4 & .18 & .10 & .62 & .23 & -- & -- & -- & .44 & $<.05$ & -- & $<.05$ & .15 & -- & -- \\
\hline $06 / 07 / 95$ & 1445 & $\mathrm{R}$ & .20 & .07 & .65 & 2.0 & .31 & .16 & .89 & $<.05$ & -- & -- & -- & .61 & $<.05$ & -- & $<.05$ & .22 & -- & -- \\
\hline $06 / 15 / 95$ & 1410 & $\mathrm{R}$ & .28 & .07 & .86 & 2.3 & .34 & .19 & 1.2 & .56 & -- & -- & -- & .73 & $<.05$ & -- & $<.05$ & .20 & -- & -- \\
\hline $06 / 15 / 95$ & 1410 & $\mathrm{~L}$ & .22 & .06 & .93 & 2.0 & .30 & .16 & 1.0 & .46 & -- & -- & -- & .64 & $<.05$ & -- & $<.05$ & .17 & -- & -- \\
\hline $06 / 23 / 95$ & 1315 & $\mathrm{R}$ & .21 & .07 & .47 & 2.3 & .27 & .36 & 1.4 & .60 & $<0.05$ & -- & -- & .68 & $<.05$ & -- & $<.05$ & .14 & -- & -- \\
\hline $07 / 03 / 95$ & 1400 & $\mathrm{R}$ & .10 & .05 & .52 & 1.7 & .22 & .30 & .92 & .49 & $<.05$ & -- & -- & .54 & $<.05$ & -- & $<.05$ & .69 & -- & -- \\
\hline $07 / 06 / 95$ & 1030 & $\mathrm{R}$ & .08 & .05 & .67 & 1.5 & .22 & .14 & .75 & .43 & $<.05$ & -- & -- & .48 & $<.05$ & -- & $<.05$ & .07 & -- & -- \\
\hline
\end{tabular}


Table 4. Results of analysis of selected triazine herbicides and degradation products by gas chromatography/mass spectrometry methods GCS (1993-96) and GCR (1996-2003) at the U.S. Geological Survey Organic Geochemistry Research Laboratory, Lawrence, Kansas, for water samples collected from the Mississippi River at Baton Rouge,

Louisiana, 1993-2003.-Continued

[R, regular sample; L, laboratory duplicate sample; --, no data; <, less than; ESA, ethanesulfonic acid; ELISA, enzyme-linked immunosorbent assay; ametryn, deisopropylprometryn, demethylnorflurazon, flufenacet, norflurazon, pendimethalin, propachlor, propanil, propazine, prometryn, terbutryn, and 3-trifluoromethylaniline were not detected]

\begin{tabular}{|c|c|c|c|c|c|c|c|c|c|c|c|c|c|c|c|c|c|c|c|c|}
\hline \multirow[b]{2}{*}{$\begin{array}{c}\text { Date of } \\
\text { collection } \\
\text { (month/ } \\
\text { day/year) }\end{array}$} & \multirow[b]{2}{*}{$\begin{array}{l}\text { Collec- } \\
\text { tion } \\
\text { time } \\
\text { (24- } \\
\text { hour) }\end{array}$} & \multirow[b]{2}{*}{$\begin{array}{l}\text { Sam- } \\
\text { ple } \\
\text { type }\end{array}$} & \multicolumn{18}{|c|}{ Concentration, in micrograms per liter } \\
\hline & & & $\begin{array}{l}\text { Aceto- } \\
\text { chlor }\end{array}$ & $\begin{array}{l}\text { Ala- } \\
\text { chlor }\end{array}$ & $\begin{array}{l}\text { Ala- } \\
\text { chlor } \\
\text { ESA } \\
\text { ELISA }\end{array}$ & $\begin{array}{l}\text { Atra- } \\
\text { zine }\end{array}$ & $\begin{array}{l}\text { De- } \\
\text { ethyl- } \\
\text { atra- } \\
\text { zine }\end{array}$ & $\begin{array}{l}\text { Deiso- } \\
\text { propyl- } \\
\text { atrazine }\end{array}$ & $\begin{array}{l}\text { Cyan- } \\
\text { azine }\end{array}$ & $\begin{array}{l}\text { Cyan- } \\
\text { azine } \\
\text { amide }\end{array}$ & $\begin{array}{c}\text { De- } \\
\text { methyl- } \\
\text { fluo- } \\
\text { metu- } \\
\text { ron }\end{array}$ & $\begin{array}{c}\text { Di- } \\
\text { meth- } \\
\text { enamid }\end{array}$ & $\begin{array}{l}\text { Fluo- } \\
\text { met- } \\
\text { uron }\end{array}$ & $\begin{array}{l}\text { Meto- } \\
\text { lachlor }\end{array}$ & $\begin{array}{l}\text { Metri- } \\
\text { buzin }\end{array}$ & $\begin{array}{l}\text { Moli- } \\
\text { nate }\end{array}$ & $\begin{array}{l}\text { Pro- } \\
\text { meton }\end{array}$ & $\begin{array}{l}\text { Sima- } \\
\text { zine }\end{array}$ & $\begin{array}{c}\text { Tri- } \\
\text { fluralin }\end{array}$ & $\begin{array}{c}\text { 3-triflu- } \\
\text { oro- } \\
\text { methyl- } \\
\text { phenyl- } \\
\text { urea }\end{array}$ \\
\hline $07 / 06 / 95$ & 1030 & $\mathrm{~L}$ & 0.07 & $<0.05$ & 0.72 & 1.5 & 0.21 & 0.13 & 0.68 & 0.42 & $<0.05$ & -- & -- & 0.48 & $<0.05$ & -- & $<0.05$ & 0.10 & -- & -- \\
\hline 07/20/95 & 1430 & $\mathrm{R}$ & $<.05$ & $<.05$ & .83 & 1.3 & .21 & .12 & .68 & .36 & $<.05$ & -- & $<0.05$ & .44 & $<.05$ & $<0.05$ & $<.05$ & .05 & -- & $<0.05$ \\
\hline $07 / 27 / 95$ & 1150 & $\mathrm{R}$ & $<.05$ & $<.05$ & .75 & 1.6 & .27 & .18 & .88 & .55 & $<.05$ & -- & $<.05$ & .44 & $<.05$ & $<.05$ & $<.05$ & .06 & -- & $<.05$ \\
\hline 08/02/95 & 1400 & $\mathrm{R}$ & $<.05$ & $<.05$ & .67 & .97 & .15 & $<.05$ & .35 & .13 & $<.05$ & -- & $<.05$ & .27 & $<.05$ & $<.05$ & $<.05$ & $<.05$ & $<0.05$ & $<.05$ \\
\hline 08/11/95 & 1100 & $\mathrm{R}$ & $<.05$ & $<.05$ & .64 & .63 & .13 & .07 & .19 & .10 & $<.05$ & -- & $<.05$ & .20 & $<.05$ & $<.05$ & $<.05$ & $<.05$ & $<.05$ & $<.05$ \\
\hline 08/18/95 & 1300 & $\mathrm{R}$ & $<.05$ & $<.05$ & .74 & .69 & .14 & .47 & .20 & .13 & $<.05$ & -- & $<.05$ & .22 & $<.05$ & $<.05$ & $<.05$ & $<.05$ & $<.05$ & $<.05$ \\
\hline 08/18/95 & 1300 & $\mathrm{~L}$ & $<.05$ & $<.05$ & .93 & .71 & .14 & .33 & .20 & .10 & $<.05$ & -- & $<.05$ & .23 & $<.05$ & $<.05$ & $<.05$ & .05 & $<.05$ & $<.05$ \\
\hline 08/25/95 & 1400 & $\mathrm{R}$ & $<.05$ & $<.05$ & .94 & .65 & .15 & .12 & .17 & $<.05$ & $<.05$ & -- & $<.05$ & .23 & $<.05$ & $<.05$ & $<.05$ & $<.05$ & $<.05$ & $<.05$ \\
\hline 09/04/95 & 1430 & $\mathrm{R}$ & $<.05$ & $<.05$ & 1.1 & .57 & .15 & .10 & .13 & $<.05$ & $<.05$ & -- & $<.05$ & .15 & $<.05$ & $<.05$ & $<.05$ & $<.05$ & $<.05$ & $<.05$ \\
\hline 09/07/95 & 1330 & $\mathrm{R}$ & $<.05$ & $<.05$ & 1.2 & .52 & .13 & .10 & .11 & $<.05$ & $<.05$ & -- & $<.05$ & .19 & $<.05$ & $<.05$ & $<.05$ & $<.05$ & $<.05$ & $<.05$ \\
\hline 09/15/95 & 1100 & $\mathrm{R}$ & $<.05$ & $<.05$ & 1.2 & .45 & .11 & .08 & .10 & $<.05$ & $<.05$ & -- & $<.05$ & .14 & $<.05$ & $<.05$ & $<.05$ & $<.05$ & $<.05$ & $<.05$ \\
\hline 09/22/95 & 1400 & $\mathrm{R}$ & $<.05$ & $<.05$ & .99 & .24 & .07 & .06 & .05 & $<.05$ & $<.05$ & -- & $<.05$ & .06 & $<.05$ & $<.05$ & $<.05$ & $<.05$ & $<.05$ & $<.05$ \\
\hline 09/22/95 & 1400 & $\mathrm{~L}$ & $<.05$ & $<.05$ & .87 & .29 & .09 & .06 & .05 & $<.05$ & $<.05$ & -- & $<.05$ & .07 & $<.05$ & $<.05$ & $<.05$ & $<.05$ & $<.05$ & $<.05$ \\
\hline 09/28/95 & 1330 & $\mathrm{R}$ & $<.05$ & $<.05$ & .59 & .20 & .06 & $<.05$ & $<.05$ & $<.05$ & $<.05$ & -- & $<.05$ & .05 & $<.05$ & $<.05$ & $<.05$ & $<.05$ & $<.05$ & $<.05$ \\
\hline $01 / 12 / 96$ & 1300 & $\mathrm{R}$ & $<.05$ & $<.05$ & .40 & .12 & $<.05$ & $<.05$ & $<.05$ & $<.05$ & $<.05$ & -- & $<.05$ & .06 & $<.05$ & $<.05$ & $<.05$ & $<.05$ & $<.05$ & $<.05$ \\
\hline $01 / 12 / 96$ & 1300 & $\mathrm{~L}$ & $<.05$ & $<.05$ & .45 & .11 & $<.05$ & $<.05$ & $<.05$ & $<.05$ & $<.05$ & -- & $<.05$ & .06 & $<.05$ & $<.05$ & $<.05$ & $<.05$ & $<.05$ & $<.05$ \\
\hline $01 / 31 / 96$ & 1330 & $\mathrm{R}$ & $<.05$ & $<.05$ & .33 & .10 & $<.05$ & $<.05$ & $<.05$ & $<.05$ & $<.05$ & -- & $<.05$ & $<.05$ & $<.05$ & $<.05$ & $<.05$ & $<.05$ & $<.05$ & $<.05$ \\
\hline $01 / 31 / 96$ & 1330 & $\mathrm{~L}$ & $<.05$ & $<.05$ & .28 & .10 & $<.05$ & $<.05$ & $<.05$ & $<.05$ & $<.05$ & -- & $<.05$ & $<.05$ & $<.05$ & $<.05$ & $<.05$ & $<.05$ & $<.05$ & $<.05$ \\
\hline 02/16/96 & 1100 & $\mathrm{R}$ & $<.05$ & $<.05$ & .33 & .11 & $<.05$ & $<.05$ & $<.05$ & $<.05$ & $<.05$ & -- & $<.05$ & $<.05$ & $<.05$ & $<.05$ & $<.05$ & $<.05$ & $<.05$ & $<.05$ \\
\hline 02/29/96 & 1130 & $\mathrm{R}$ & $<.05$ & $<.05$ & .25 & .11 & $<.05$ & $<.05$ & $<.05$ & $<.05$ & $<.05$ & -- & $<.05$ & $<.05$ & $<.05$ & $<.05$ & $<.05$ & $<.05$ & $<.05$ & $<.05$ \\
\hline
\end{tabular}


Table 4. Results of analysis of selected triazine herbicides and degradation products by gas chromatography/mass spectrometry methods GCS (1993-96) and GCR (1996-2003) at the U.S. Geological Survey Organic Geochemistry Research Laboratory, Lawrence, Kansas, for water samples collected from the Mississippi River at Baton Rouge,

Louisiana, 1993-2003.-Continued

[R, regular sample; L, laboratory duplicate sample; --, no data; <, less than; ESA, ethanesulfonic acid; ELISA, enzyme-linked immunosorbent assay; ametryn, deisopropylprometryn, demethylnorflurazon, flufenacet, norflurazon, pendimethalin, propachlor, propanil, propazine, prometryn, terbutryn, and 3-trifluoromethylaniline were not detected]

\begin{tabular}{|c|c|c|c|c|c|c|c|c|c|c|c|c|c|c|c|c|c|c|c|c|}
\hline \multirow[b]{2}{*}{$\begin{array}{c}\text { Date of } \\
\text { collection } \\
\text { (month/ } \\
\text { day/year) }\end{array}$} & \multirow[b]{2}{*}{$\begin{array}{l}\text { Collec- } \\
\text { tion } \\
\text { time } \\
\text { (24- } \\
\text { hour) }\end{array}$} & \multirow[b]{2}{*}{$\begin{array}{l}\text { Sam- } \\
\text { ple } \\
\text { type }\end{array}$} & \multicolumn{18}{|c|}{ Concentration, in micrograms per liter } \\
\hline & & & $\begin{array}{c}\text { Aceto- } \\
\text { chlor }\end{array}$ & $\begin{array}{l}\text { Ala- } \\
\text { chlor }\end{array}$ & $\begin{array}{l}\text { Ala- } \\
\text { chlor } \\
\text { ESA } \\
\text { ELISA }\end{array}$ & $\begin{array}{l}\text { Atra- } \\
\text { zine }\end{array}$ & $\begin{array}{l}\text { De- } \\
\text { ethyl- } \\
\text { atra- } \\
\text { zine }\end{array}$ & $\begin{array}{l}\text { Deiso- } \\
\text { propyl- } \\
\text { atrazine }\end{array}$ & $\begin{array}{l}\text { Cyan- } \\
\text { azine }\end{array}$ & $\begin{array}{l}\text { Cyan- } \\
\text { azine } \\
\text { amide }\end{array}$ & $\begin{array}{c}\text { De- } \\
\text { methyl- } \\
\text { fluo- } \\
\text { metu- } \\
\text { ron }\end{array}$ & $\begin{array}{l}\text { Di- } \\
\text { meth- } \\
\text { enamid }\end{array}$ & $\begin{array}{l}\text { Fluo- } \\
\text { met- } \\
\text { uron }\end{array}$ & $\begin{array}{l}\text { Meto- } \\
\text { lachlor }\end{array}$ & $\begin{array}{l}\text { Metri- } \\
\text { buzin }\end{array}$ & $\begin{array}{l}\text { Moli- } \\
\text { nate }\end{array}$ & $\begin{array}{l}\text { Pro- } \\
\text { meton }\end{array}$ & $\begin{array}{l}\text { Sima- } \\
\text { zine }\end{array}$ & $\begin{array}{c}\text { Tri- } \\
\text { fluralin }\end{array}$ & $\begin{array}{l}\text { 3-triflu- } \\
\text { oro- } \\
\text { methyl- } \\
\text { phenyl- } \\
\text { urea }\end{array}$ \\
\hline $03 / 08 / 96$ & 1400 & $\mathrm{R}$ & $<0.05$ & $<0.05$ & 0.32 & 0.13 & $<0.05$ & $<0.05$ & $<0.05$ & $<0.05$ & $<0.05$ & -- & $<0.05$ & 0.10 & $<0.05$ & $<0.05$ & $<0.05$ & $<0.05$ & $<0.05$ & $<0.05$ \\
\hline $03 / 15 / 96$ & 1430 & $\mathrm{R}$ & $<.05$ & $<.05$ & .26 & .14 & $<.05$ & $<.05$ & $<.05$ & $<.05$ & $<.05$ & -- & $<.05$ & .11 & $<.05$ & $<.05$ & $<.05$ & $<.05$ & $<.05$ & $<.05$ \\
\hline $03 / 15 / 96$ & 1430 & $\mathrm{~L}$ & $<.05$ & $<.05$ & .22 & .12 & $<.05$ & $<.05$ & $<.05$ & $<.05$ & $<.05$ & -- & $<.05$ & .09 & $<.05$ & $<.05$ & $<.05$ & $<.05$ & $<.05$ & $<.05$ \\
\hline $03 / 22 / 96$ & 1330 & $\mathrm{R}$ & $<.05$ & $<.05$ & .24 & .12 & $<.05$ & $<.05$ & $<.05$ & $<.05$ & $<.05$ & -- & $<.05$ & .09 & $<.05$ & $<.05$ & $<.05$ & $<.05$ & $<.05$ & $<.05$ \\
\hline $04 / 05 / 96$ & 1230 & $\mathrm{R}$ & $<.05$ & $<.05$ & .43 & .17 & $<.05$ & $<.05$ & $<.05$ & $<.05$ & $<.05$ & -- & $<.05$ & .10 & $<.05$ & $<.05$ & $<.05$ & $<.05$ & $<.05$ & $<.05$ \\
\hline $04 / 12 / 96$ & 1300 & $\mathrm{R}$ & $<.05$ & $<.05$ & .32 & .17 & $<.05$ & $<.05$ & $<.05$ & $<.05$ & $<.05$ & -- & $<.05$ & .09 & $<.05$ & $<.05$ & $<.05$ & $<.05$ & $<.05$ & $<.05$ \\
\hline $04 / 12 / 96$ & 1300 & $\mathrm{~L}$ & $<.05$ & $<.05$ & .37 & .18 & $<.05$ & $<.05$ & $<.05$ & $<.05$ & $<.05$ & -- & $<.05$ & .10 & $<.05$ & $<.05$ & $<.05$ & $<.05$ & $<.05$ & $<.05$ \\
\hline $04 / 26 / 96$ & 1400 & $\mathrm{R}$ & $<.05$ & $<.05$ & .33 & .76 & .07 & $<.05$ & $<.05$ & $<.05$ & $<.05$ & -- & $<.05$ & .48 & .05 & $<.05$ & $<.05$ & $<.05$ & $<.05$ & $<.05$ \\
\hline 05/03/96 & 1030 & $\mathrm{R}$ & .09 & .05 & .33 & 1.0 & .08 & .06 & .07 & $<.05$ & $<.05$ & -- & $<.05$ & .44 & $<.05$ & $<.05$ & $<.05$ & $<.05$ & $<.05$ & $<.05$ \\
\hline $05 / 10 / 96$ & 1830 & $\mathrm{R}$ & .16 & $<.05$ & .37 & 1.8 & .12 & .08 & .32 & .08 & $<.05$ & -- & $<.05$ & .54 & $<.05$ & $<.05$ & $<.05$ & .21 & $<.05$ & $<.05$ \\
\hline $05 / 17 / 96$ & 1900 & $\mathrm{R}$ & .12 & $<.05$ & .39 & 1.8 & .14 & .10 & .31 & .11 & $<.05$ & -- & $<.05$ & .56 & $<.05$ & $<.05$ & $<.05$ & .21 & $<.05$ & $<.05$ \\
\hline $05 / 23 / 96$ & 1700 & $\mathrm{R}$ & .21 & $<.05$ & .46 & 3.0 & .23 & .13 & .85 & .32 & $<.05$ & -- & $<.05$ & .80 & $<.05$ & $<.05$ & $<.05$ & .17 & $<.05$ & $<.05$ \\
\hline $05 / 31 / 96$ & 1000 & $\mathrm{R}$ & .34 & $<.05$ & .57 & 3.3 & .28 & .15 & 1.0 & .40 & $<.05$ & -- & $<.05$ & 1.1 & $<.05$ & $<.05$ & $<.05$ & .14 & $<.05$ & $<.05$ \\
\hline 06/06/96 & 1025 & $\mathrm{R}$ & .17 & $<.05$ & .58 & 2.2 & .22 & .12 & .56 & .31 & $<.05$ & -- & $<.05$ & .71 & $<.05$ & $<.05$ & $<.05$ & .11 & $<.05$ & $<.05$ \\
\hline 06/06/96 & 1025 & $\mathrm{~L}$ & .18 & $<.05$ & .50 & 2.3 & .24 & .14 & .58 & .31 & $<.05$ & -- & $<.05$ & .74 & $<.05$ & $<.05$ & $<.05$ & .11 & $<.05$ & $<.05$ \\
\hline $07 / 06 / 96$ & 1030 & $\mathrm{R}$ & .13 & .06 & 1.2 & 2.1 & .40 & .47 & .67 & .44 & $<.05$ & -- & $<.05$ & .83 & $<.05$ & $<.05$ & $<.05$ & .15 & $<.05$ & $<.05$ \\
\hline 07/10/96 & 1030 & $\mathrm{R}$ & .11 & .05 & 1.1 & 1.1 & .38 & .21 & .40 & .43 & $<.05$ & -- & $<.05$ & .75 & $<.05$ & $<.05$ & $<.05$ & .08 & $<.05$ & $<.05$ \\
\hline 07/10/96 & 1030 & $\mathrm{~L}$ & .12 & .05 & 1.4 & 1.1 & .38 & .09 & .39 & .41 & $<.05$ & -- & $<.05$ & .74 & $<.05$ & $<.05$ & $<.05$ & .07 & $<.05$ & $<.05$ \\
\hline $07 / 18 / 96$ & 1035 & $\mathrm{R}$ & $<.05$ & .05 & .80 & .70 & .39 & .25 & .43 & .79 & $<.05$ & -- & $<.05$ & .90 & $<.05$ & $<.05$ & -- & .08 & $<.05$ & $<.05$ \\
\hline $08 / 02 / 96$ & 1140 & $\mathrm{R}$ & $<.05$ & $<.05$ & .51 & .86 & .22 & .12 & .35 & $<.05$ & $<.05$ & -- & $<.05$ & .52 & $<.05$ & $<.05$ & -- & .08 & $<.05$ & $<.05$ \\
\hline
\end{tabular}


Table 4. Results of analysis of selected triazine herbicides and degradation products by gas chromatography/mass spectrometry methods GCS (1993-96) and GCR (1996-2003) at the U.S. Geological Survey Organic Geochemistry Research Laboratory, Lawrence, Kansas, for water samples collected from the Mississippi River at Baton Rouge,

Louisiana, 1993-2003.-Continued

[R, regular sample; L, laboratory duplicate sample; --, no data; <, less than; ESA, ethanesulfonic acid; ELISA, enzyme-linked immunosorbent assay; ametryn, deisopropylprometryn, demethylnorflurazon, flufenacet, norflurazon, pendimethalin, propachlor, propanil, propazine, prometryn, terbutryn, and 3-trifluoromethylaniline were not detected]

\begin{tabular}{|c|c|c|c|c|c|c|c|c|c|c|c|c|c|c|c|c|c|c|c|c|}
\hline \multirow[b]{2}{*}{$\begin{array}{l}\text { Date of } \\
\text { collection } \\
\text { (month/ } \\
\text { day/year) }\end{array}$} & \multirow[b]{2}{*}{$\begin{array}{l}\text { Collec- } \\
\text { tion } \\
\text { time } \\
\text { (24- } \\
\text { hour) }\end{array}$} & \multirow[b]{2}{*}{$\begin{array}{l}\text { Sam- } \\
\text { ple } \\
\text { type }\end{array}$} & \multicolumn{18}{|c|}{ Concentration, in micrograms per liter } \\
\hline & & & $\begin{array}{c}\text { Aceto- } \\
\text { chlor }\end{array}$ & $\begin{array}{l}\text { Ala- } \\
\text { chlor }\end{array}$ & $\begin{array}{l}\text { Ala- } \\
\text { chlor } \\
\text { ESA } \\
\text { ELISA }\end{array}$ & $\begin{array}{l}\text { Atra- } \\
\text { zine }\end{array}$ & $\begin{array}{l}\text { De- } \\
\text { ethyl- } \\
\text { atra- } \\
\text { zine }\end{array}$ & $\begin{array}{l}\text { Deiso- } \\
\text { propyl- } \\
\text { atrazine }\end{array}$ & $\begin{array}{l}\text { Cyan- } \\
\text { azine }\end{array}$ & $\begin{array}{l}\text { Cyan- } \\
\text { azine } \\
\text { amide }\end{array}$ & $\begin{array}{c}\text { De- } \\
\text { methyl- } \\
\text { fluo- } \\
\text { metu- } \\
\text { ron }\end{array}$ & $\begin{array}{c}\text { Di- } \\
\text { meth- } \\
\text { enamid }\end{array}$ & $\begin{array}{l}\text { Fluo- } \\
\text { met- } \\
\text { uron }\end{array}$ & $\begin{array}{l}\text { Meto- } \\
\text { lachlor }\end{array}$ & $\begin{array}{l}\text { Metri- } \\
\text { buzin }\end{array}$ & $\begin{array}{l}\text { Moli- } \\
\text { nate }\end{array}$ & $\begin{array}{l}\text { Pro- } \\
\text { meton }\end{array}$ & $\begin{array}{l}\text { Sima- } \\
\text { zine }\end{array}$ & $\begin{array}{c}\text { Tri- } \\
\text { fluralin }\end{array}$ & $\begin{array}{c}\text { 3-triflu- } \\
\text { oro- } \\
\text { methyl- } \\
\text { phenyl- } \\
\text { urea }\end{array}$ \\
\hline $08 / 09 / 96$ & 1105 & $\mathrm{R}$ & $<0.05$ & $<0.05$ & 0.33 & 0.80 & 0.21 & 0.13 & 0.42 & 0.22 & $<0.05$ & -- & $<0.05$ & 0.50 & $<0.05$ & $<0.05$ & -- & 0.08 & $<0.05$ & $<0.05$ \\
\hline 08/16/96 & 1130 & $\mathrm{R}$ & $<.05$ & $<.05$ & .44 & .74 & .20 & .12 & .31 & .17 & $<.05$ & -- & $<.05$ & .44 & $<.05$ & $<.05$ & -- & .06 & $<.05$ & $<.05$ \\
\hline 08/30/96 & 1240 & $\mathrm{R}$ & $<.05$ & $<.05$ & .59 & .46 & .16 & .12 & .16 & $<.05$ & $<.05$ & -- & $<.05$ & .23 & $<.05$ & $<.05$ & -- & $<.05$ & $<.05$ & $<.05$ \\
\hline 08/30/96 & 1240 & $\mathrm{R}$ & $<.05$ & $<.05$ & .56 & .48 & .17 & .11 & .18 & $<.05$ & $<.05$ & -- & $<.05$ & .24 & $<.05$ & $<.05$ & -- & .05 & $<.05$ & $<.05$ \\
\hline 09/30/96 & 1500 & $\mathrm{R}$ & $<.05$ & $<.05$ & .35 & .20 & .08 & .05 & .07 & $<.05$ & $<.05$ & -- & $<.05$ & .10 & $<.05$ & $<.05$ & -- & $<.05$ & $<.05$ & $<.05$ \\
\hline $10 / 30 / 96$ & 1330 & $\mathrm{R}$ & $<.05$ & $<.05$ & .25 & .15 & .06 & $<.05$ & $<.05$ & $<.05$ & $<.05$ & -- & $<.05$ & .07 & $<.05$ & $<.05$ & -- & $<.05$ & $<.05$ & $<.05$ \\
\hline $11 / 21 / 96$ & 1040 & $\mathrm{R}$ & $<.05$ & $<.05$ & .25 & .17 & .08 & $<.05$ & $<.05$ & $<.05$ & $<.05$ & -- & $<.05$ & .08 & $<.05$ & $<.05$ & -- & $<.05$ & $<.05$ & $<.05$ \\
\hline $12 / 03 / 96$ & -- & $\mathrm{R}$ & $<.05$ & $<.05$ & $<.10$ & .09 & $<.05$ & $<.05$ & $<.05$ & $<.05$ & $<.05$ & -- & $<.05$ & $<.05$ & $<.05$ & $<.05$ & -- & $<.05$ & $<.05$ & \\
\hline $12 / 11 / 96$ & 1545 & $\mathrm{R}$ & $<.05$ & $<.05$ & .30 & .12 & .05 & $<.05$ & $<.05$ & $<.05$ & $<.05$ & -- & $<.05$ & .05 & $<.05$ & $<.05$ & -- & $<.05$ & $<.05$ & $<.05$ \\
\hline $02 / 14 / 97$ & 1130 & $\mathrm{R}$ & $<.05$ & $<.05$ & .38 & .07 & $<.05$ & $<.05$ & $<.05$ & $<.05$ & $<.05$ & -- & $<.05$ & .05 & $<.05$ & $<.05$ & -- & $<.05$ & $<.05$ & $<.05$ \\
\hline 03/05/97 & 1330 & $\mathrm{R}$ & $<.05$ & $<.05$ & .39 & .10 & $<.05$ & $<.05$ & $<.05$ & $<.05$ & $<.05$ & -- & $<.05$ & .07 & $<.05$ & $<.05$ & -- & $<.05$ & $<.05$ & $<.05$ \\
\hline $03 / 22 / 97$ & 1130 & $\mathrm{R}$ & $<.05$ & $<.05$ & .28 & .08 & $<.05$ & $<.05$ & $<.05$ & $<.05$ & $<.05$ & -- & $<.05$ & .09 & $<.05$ & $<.05$ & -- & $<.05$ & $<.05$ & $<.05$ \\
\hline 03/27/97 & 1000 & $\mathrm{R}$ & $<.05$ & $<.05$ & .36 & .07 & $<.05$ & $<.05$ & $<.05$ & $<.05$ & $<.05$ & -- & $<.05$ & .08 & $<.05$ & $<.05$ & -- & $<.05$ & $<.05$ & $<.05$ \\
\hline 04/03/97 & 1010 & $\mathrm{R}$ & $<.05$ & $<.05$ & .40 & .08 & .05 & $<.05$ & $<.05$ & $<.05$ & $<.05$ & -- & $<.05$ & .10 & $<.05$ & $<.05$ & -- & $<.05$ & $<.05$ & $<.05$ \\
\hline $04 / 17 / 97$ & 1505 & $\mathrm{R}$ & .13 & $<.05$ & .34 & .90 & .06 & .06 & $<.05$ & $<.05$ & $<.05$ & -- & $<.05$ & .39 & $<.05$ & $<.05$ & -- & .06 & $<.05$ & $<.05$ \\
\hline 04/30/97 & 1600 & $\mathrm{R}$ & .06 & $<.05$ & .38 & .71 & .06 & .06 & $<.05$ & $<.05$ & $<.05$ & -- & $<.05$ & .33 & $<.05$ & $<.05$ & -- & $<.05$ & $<.05$ & $<.05$ \\
\hline $05 / 16 / 97$ & 0615 & $\mathrm{R}$ & .12 & $<.05$ & .46 & 1.1 & .11 & .06 & $<.05$ & $<.05$ & $<.05$ & -- & $<.05$ & .60 & $<.05$ & $<.05$ & -- & .17 & $<.05$ & $<.05$ \\
\hline 05/27/97 & 1255 & $\mathrm{R}$ & .32 & .05 & .42 & 1.8 & .14 & .09 & .81 & .22 & $<.05$ & -- & .06 & .85 & $<.05$ & $<.05$ & -- & .09 & $<.05$ & $<.05$ \\
\hline 06/06/97 & 1115 & $\mathrm{R}$ & .10 & $<.05$ & $<.10$ & .85 & .07 & .12 & $<.05$ & $<.05$ & $<.05$ & -- & .17 & .54 & $<.05$ & $<.05$ & -- & .05 & $<.05$ & $<.05$ \\
\hline
\end{tabular}


Table 4. Results of analysis of selected triazine herbicides and degradation products by gas chromatography/mass spectrometry methods GCS (1993-96) and GCR (1996-2003) at the U.S. Geological Survey Organic Geochemistry Research Laboratory, Lawrence, Kansas, for water samples collected from the Mississippi River at Baton Rouge,

Louisiana, 1993-2003.-Continued

$[R$, regular sample; L, laboratory duplicate sample; --, no data; <, less than; ESA, ethanesulfonic acid; ELISA, enzyme-linked immunosorbent assay; ametryn, deisopropylprometryn, demethylnorflurazon, flufenacet, norflurazon, pendimethalin, propachlor, propanil, propazine, prometryn, terbutryn, and 3-trifluoromethylaniline were not detected]

\begin{tabular}{|c|c|c|c|c|c|c|c|c|c|c|c|c|c|c|c|c|c|c|c|c|}
\hline \multirow[b]{2}{*}{$\begin{array}{l}\text { Date of } \\
\text { collection } \\
\text { (month/ } \\
\text { day/year) }\end{array}$} & \multirow[b]{2}{*}{$\begin{array}{l}\text { Collec- } \\
\text { tion } \\
\text { time } \\
(24- \\
\text { hour) }\end{array}$} & \multirow[b]{2}{*}{$\begin{array}{l}\text { Sam- } \\
\text { ple } \\
\text { type }\end{array}$} & \multicolumn{18}{|c|}{ Concentration, in micrograms per liter } \\
\hline & & & $\begin{array}{l}\text { Aceto- } \\
\text { chlor }\end{array}$ & $\begin{array}{l}\text { Ala- } \\
\text { chlor }\end{array}$ & $\begin{array}{l}\text { Ala- } \\
\text { chlor } \\
\text { ESA } \\
\text { ELISA }\end{array}$ & $\begin{array}{l}\text { Atra- } \\
\text { zine }\end{array}$ & $\begin{array}{l}\text { De- } \\
\text { ethyl- } \\
\text { atra- } \\
\text { zine }\end{array}$ & $\begin{array}{l}\text { Deiso- } \\
\text { propyl- } \\
\text { atrazine }\end{array}$ & $\begin{array}{l}\text { Cyan- } \\
\text { azine }\end{array}$ & $\begin{array}{l}\text { Cyan- } \\
\text { azine } \\
\text { amide }\end{array}$ & $\begin{array}{c}\text { De- } \\
\text { methyl- } \\
\text { fluo- } \\
\text { metu- } \\
\text { ron }\end{array}$ & $\begin{array}{l}\text { Di- } \\
\text { meth- } \\
\text { enamid }\end{array}$ & $\begin{array}{l}\text { Fluo- } \\
\text { met- } \\
\text { uron }\end{array}$ & $\begin{array}{l}\text { Meto- } \\
\text { lachlor }\end{array}$ & $\begin{array}{l}\text { Metri- } \\
\text { buzin }\end{array}$ & $\begin{array}{l}\text { Moli- } \\
\text { nate }\end{array}$ & $\begin{array}{l}\text { Pro- } \\
\text { meton }\end{array}$ & $\begin{array}{l}\text { Sima- } \\
\text { zine }\end{array}$ & $\begin{array}{c}\text { Tri- } \\
\text { fluralin }\end{array}$ & $\begin{array}{l}\text { 3-triflu- } \\
\text { oro- } \\
\text { methyl- } \\
\text { phenyl- } \\
\text { urea }\end{array}$ \\
\hline $06 / 06 / 97$ & 1115 & $\mathrm{~L}$ & $<0.05$ & $<0.05$ & -- & 0.93 & 0.14 & $<0.05$ & 0.32 & 0.11 & $<0.05$ & -- & 0.17 & 0.71 & 0.11 & $<0.05$ & -- & 0.06 & $<0.05$ & $<0.05$ \\
\hline $06 / 27 / 97$ & 1115 & $\mathrm{R}$ & .17 & $<.05$ & 0.86 & 2.2 & .50 & .39 & 69 & .49 & $<.05$ & -- & .09 & .92 & $<.05$ & .07 & -- & .24 & $<.05$ & $<.05$ \\
\hline 07/01/97 & 1420 & $\mathrm{R}$ & $<.05$ & $<.05$ & .85 & 2.0 & .51 & .40 & .67 & .55 & $<.05$ & -- & .08 & .79 & $<.05$ & .06 & -- & .20 & $<.05$ & $<.05$ \\
\hline $07 / 14 / 97$ & 1200 & $\mathrm{R}$ & .07 & $<.05$ & .50 & 1.6 & .35 & .08 & .46 & .31 & $<.05$ & -- & .09 & .58 & $<.05$ & .06 & -- & .06 & $<.05$ & $<.05$ \\
\hline $07 / 25 / 97$ & 1030 & $\mathrm{R}$ & $<.05$ & $<.05$ & .57 & 1.1 & .28 & $<.05$ & .54 & .14 & $<.05$ & -- & $<.05$ & .39 & $<.05$ & .10 & -- & $<.05$ & $<.05$ & $<.05$ \\
\hline $08 / 14 / 97$ & 1415 & $\mathrm{R}$ & $<.05$ & $<.05$ & 3.7 & .46 & .15 & .10 & .20 & .09 & $<.05$ & -- & $<.05$ & .21 & $<.05$ & $<.05$ & -- & $<.05$ & $<.05$ & $<.05$ \\
\hline $10 / 22 / 97$ & 1045 & $\mathrm{R}$ & $<.05$ & $<.05$ & 1.8 & .17 & .07 & $<.05$ & .11 & $<.05$ & $<.05$ & -- & $<.05$ & .10 & $<.05$ & $<.05$ & -- & $<.05$ & $<.05$ & $<.05$ \\
\hline $12 / 10 / 97$ & 1545 & $\mathrm{R}$ & $<.05$ & $<.05$ & 1.5 & .13 & .06 & $<.05$ & .10 & $<.05$ & $<.05$ & -- & $<.05$ & .07 & $<.05$ & $<.05$ & -- & $<.05$ & $<.05$ & $<.05$ \\
\hline $12 / 10 / 97$ & 1545 & $\mathrm{~L}$ & $<.05$ & $<.05$ & 1.9 & .13 & .06 & $<.05$ & .10 & $<.05$ & $<.05$ & -- & $<.05$ & .08 & $<.05$ & $<.05$ & -- & $<.05$ & $<.05$ & $<.05$ \\
\hline $01 / 14 / 98$ & 1430 & $\mathrm{R}$ & $<.05$ & $<.05$ & .16 & .14 & .05 & .05 & $<.05$ & $<.05$ & $<.05$ & -- & $<.05$ & .05 & $<.05$ & $<.05$ & -- & .07 & $<.05$ & $<.05$ \\
\hline 02/04/98 & 1015 & $\mathrm{R}$ & $<.05$ & $<.05$ & .23 & .09 & .05 & $<.05$ & $<.05$ & .05 & $<.05$ & -- & $<.05$ & .05 & $<.05$ & $<.05$ & -- & $<.05$ & $<.05$ & $<.05$ \\
\hline 02/19/98 & 1330 & $\mathrm{R}$ & $<.05$ & $<.05$ & -- & .08 & $<.05$ & $<.05$ & $<.05$ & $<.05$ & $<.05$ & -- & $<.05$ & $<.05$ & $<.05$ & $<.05$ & -- & $<.05$ & $<.05$ & $<.05$ \\
\hline 02/19/98 & 1330 & $\mathrm{~L}$ & $<.05$ & $<.05$ & .25 & .08 & $<.05$ & $<.05$ & $<.05$ & $<.05$ & $<.05$ & -- & $<.05$ & $<.05$ & $<.05$ & $<.05$ & -- & $<.05$ & $<.05$ & $<.05$ \\
\hline 03/04/98 & 1000 & $\mathrm{R}$ & $<.05$ & $<.05$ & .16 & .07 & .05 & $<.05$ & $<.05$ & $<.05$ & $<.05$ & -- & $<.05$ & $<.05$ & $<.05$ & $<.05$ & -- & $<.05$ & $<.05$ & $<.05$ \\
\hline 03/10/98 & 1000 & $\mathrm{R}$ & $<.05$ & $<.05$ & .14 & .09 & .05 & $<.05$ & $<.05$ & $<.05$ & $<.05$ & -- & $<.05$ & .07 & $<.05$ & $<.05$ & -- & $<.05$ & $<.05$ & $<.05$ \\
\hline 03/20/98 & 1010 & $\mathrm{R}$ & $<.05$ & $<.05$ & .22 & .09 & .05 & $<.05$ & $<.05$ & $<.05$ & $<.05$ & -- & $<.05$ & .14 & $<.05$ & $<.05$ & -- & $<.05$ & $<.05$ & $<.05$ \\
\hline 03/27/98 & 1030 & $\mathrm{R}$ & $<.05$ & $<.05$ & .02 & .12 & .05 & $<.05$ & $<.05$ & $<.05$ & $<.05$ & -- & $<.05$ & .09 & $<.05$ & $<.05$ & -- & $<.05$ & $<.05$ & $<.05$ \\
\hline 04/10/98 & 1400 & $\mathrm{R}$ & $<.05$ & $<.05$ & .35 & .13 & .07 & $<.05$ & $<.05$ & $<.05$ & $<.05$ & -- & $<.05$ & .12 & $<.05$ & $<.05$ & -- & $<.05$ & $<.05$ & $<.05$ \\
\hline 04/17/98 & 1200 & $\mathrm{R}$ & $<.05$ & $<.05$ & .27 & .16 & .07 & .05 & .05 & $<.05$ & $<.05$ & -- & $<.05$ & .13 & $<.05$ & $<.05$ & -- & $<.05$ & $<.05$ & $<.05$ \\
\hline $04 / 22 / 98$ & 1040 & $\mathrm{R}$ & $<.05$ & $<.05$ & .47 & .17 & .09 & $<.05$ & $<.05$ & $<.05$ & $<.05$ & -- & $<.05$ & .13 & $<.05$ & $<.05$ & -- & $<.05$ & $<.05$ & $<.05$ \\
\hline
\end{tabular}


Table 4. Results of analysis of selected triazine herbicides and degradation products by gas chromatography/mass spectrometry methods GCS (1993-96) and GCR (1996-2003) at the U.S. Geological Survey Organic Geochemistry Research Laboratory, Lawrence, Kansas, for water samples collected from the Mississippi River at Baton Rouge,

Louisiana, 1993-2003.-Continued

[R, regular sample; L, laboratory duplicate sample; --, no data; <, less than; ESA, ethanesulfonic acid; ELISA, enzyme-linked immunosorbent assay; ametryn, deisopropylprometryn, demethylnorflurazon, flufenacet, norflurazon, pendimethalin, propachlor, propanil, propazine, prometryn, terbutryn, and 3-trifluoromethylaniline were not detected]

\begin{tabular}{|c|c|c|c|c|c|c|c|c|c|c|c|c|c|c|c|c|c|c|c|c|}
\hline \multirow[b]{2}{*}{$\begin{array}{c}\text { Date of } \\
\text { collection } \\
\text { (month/ } \\
\text { day/year) }\end{array}$} & \multirow[b]{2}{*}{$\begin{array}{l}\text { Collec- } \\
\text { tion } \\
\text { time } \\
\text { (24- } \\
\text { hour) }\end{array}$} & \multirow[b]{2}{*}{$\begin{array}{l}\text { Sam- } \\
\text { ple } \\
\text { type }\end{array}$} & \multicolumn{18}{|c|}{ Concentration, in micrograms per liter } \\
\hline & & & $\begin{array}{l}\text { Aceto- } \\
\text { chlor }\end{array}$ & $\begin{array}{l}\text { Ala- } \\
\text { chlor }\end{array}$ & $\begin{array}{l}\text { Ala- } \\
\text { chlor } \\
\text { ESA } \\
\text { ELISA }\end{array}$ & $\begin{array}{l}\text { Atra- } \\
\text { zine }\end{array}$ & $\begin{array}{l}\text { De- } \\
\text { ethyl- } \\
\text { atra- } \\
\text { zine }\end{array}$ & $\begin{array}{l}\text { Deiso- } \\
\text { propyl- } \\
\text { atrazine }\end{array}$ & $\begin{array}{l}\text { Cyan- } \\
\text { azine }\end{array}$ & $\begin{array}{l}\text { Cyan- } \\
\text { azine } \\
\text { amide }\end{array}$ & $\begin{array}{c}\text { De- } \\
\text { methyl- } \\
\text { fluo- } \\
\text { metu- } \\
\text { ron }\end{array}$ & $\begin{array}{c}\text { Di- } \\
\text { meth- } \\
\text { enamid }\end{array}$ & $\begin{array}{l}\text { Fluo- } \\
\text { met- } \\
\text { uron }\end{array}$ & $\begin{array}{l}\text { Meto- } \\
\text { lachlor }\end{array}$ & $\begin{array}{l}\text { Metri- } \\
\text { buzin }\end{array}$ & $\begin{array}{c}\text { Moli- } \\
\text { nate }\end{array}$ & $\begin{array}{l}\text { Pro- } \\
\text { meton }\end{array}$ & $\begin{array}{l}\text { Sima- } \\
\text { zine }\end{array}$ & $\begin{array}{c}\text { Tri- } \\
\text { fluralin }\end{array}$ & $\begin{array}{c}\text { 3-triflu- } \\
\text { oro- } \\
\text { methyl- } \\
\text { phenyl- } \\
\text { urea }\end{array}$ \\
\hline $05 / 08 / 98$ & 1200 & $\mathrm{R}$ & 0.07 & $<0.05$ & -- & 0.47 & 0.09 & 0.05 & $<0.05$ & $<0.05$ & $<0.05$ & -- & $<0.05$ & 0.25 & $<0.05$ & $<0.05$ & -- & 0.09 & $<0.05$ & $<0.05$ \\
\hline 05/08/98 & 1200 & $\mathrm{~L}$ & $<.05$ & $<.05$ & 0.32 & .53 & .09 & .05 & .08 & $<.05$ & $<.05$ & -- & $<.05$ & .22 & $<.05$ & $<.05$ & -- & .08 & $<.05$ & $<.05$ \\
\hline $07 / 24 / 98$ & 1100 & $\mathrm{R}$ & $<.05$ & $<.05$ & .86 & .99 & .43 & .24 & .36 & .48 & $<.05$ & -- & .05 & .55 & $<.05$ & $<.05$ & -- & .06 & $<.05$ & $<.05$ \\
\hline 08/07/98 & 1105 & $\mathrm{R}$ & $<.05$ & $<.05$ & .38 & .60 & .14 & .05 & .20 & .21 & $<.05$ & -- & .09 & .39 & $<.05$ & $<.05$ & -- & .05 & $<.05$ & .06 \\
\hline 08/13/98 & 1345 & $\mathrm{R}$ & $<.05$ & $<.05$ & .54 & .44 & .17 & .10 & .14 & $<.05$ & $<.05$ & -- & .06 & .22 & $<.05$ & $<.05$ & -- & $<.05$ & $<.05$ & $<.05$ \\
\hline 08/27/98 & 1120 & $\mathrm{R}$ & $<.05$ & $<.05$ & -- & .27 & .12 & $<.05$ & $<.05$ & .09 & $<.05$ & -- & $<.05$ & .17 & $<.05$ & $<.05$ & -- & $<.05$ & $<.05$ & $<.05$ \\
\hline 09/11/98 & 1535 & $\mathrm{R}$ & $<.05$ & $<.05$ & -- & .25 & .16 & .05 & .09 & .06 & $<.05$ & -- & $<.05$ & .15 & .07 & $<.05$ & -- & $<.05$ & $<.05$ & $<.05$ \\
\hline 09/18/98 & 1045 & $\mathrm{R}$ & $<.05$ & $<.05$ & -- & .23 & .14 & .08 & .06 & $<.05$ & $<.05$ & -- & $<.05$ & .11 & $<.05$ & $<.05$ & -- & $<.05$ & $<.05$ & $<.05$ \\
\hline $10 / 02 / 98$ & 1515 & $\mathrm{R}$ & .10 & $<.05$ & -- & .19 & .12 & .05 & .06 & .09 & $<.05$ & -- & $<.05$ & .09 & $<.05$ & $<.05$ & -- & $<.05$ & $<.05$ & $<.05$ \\
\hline $10 / 23 / 98$ & 1120 & $\mathrm{R}$ & $<.05$ & $<.05$ & -- & .28 & .11 & $<.05$ & .05 & $<.05$ & $<.05$ & -- & $<.05$ & .13 & $<.05$ & $<.05$ & -- & $<.05$ & $<.05$ & $<.05$ \\
\hline $10 / 30 / 98$ & 1330 & $\mathrm{R}$ & $<.05$ & $<.05$ & -- & .30 & .13 & $<.05$ & .06 & $<.05$ & $<.05$ & -- & $<.05$ & .12 & .05 & $<.05$ & -- & $<.05$ & $<.05$ & $<.05$ \\
\hline $11 / 10 / 98$ & 1430 & $\mathrm{R}$ & $<.05$ & $<.05$ & -- & .20 & .10 & .05 & $<.05$ & $<.05$ & $<.05$ & -- & $<.05$ & .09 & $<.05$ & $<.05$ & -- & $<.05$ & $<.05$ & $<.05$ \\
\hline $11 / 23 / 98$ & 0900 & $\mathrm{R}$ & $<.05$ & $<.05$ & -- & .16 & .10 & $<.05$ & $<.05$ & $<.05$ & $<.05$ & -- & $<.05$ & .07 & $<.05$ & $<.05$ & -- & $<.05$ & $<.05$ & $<.05$ \\
\hline $12 / 23 / 98$ & 0745 & $\mathrm{R}$ & $<.05$ & $<.05$ & -- & .10 & .06 & $<.05$ & $<.05$ & $<.05$ & $<.05$ & -- & $<.05$ & .06 & $<.05$ & $<.05$ & -- & $<.05$ & $<.05$ & $<.05$ \\
\hline 01/05/99 & 1100 & $\mathrm{R}$ & $<.05$ & $<.05$ & -- & .08 & .05 & $<.05$ & $<.05$ & $<.05$ & $<.05$ & -- & $<.05$ & $<.05$ & $<.05$ & $<.05$ & -- & $<.05$ & $<.05$ & $<.05$ \\
\hline 01/20/99 & 1030 & $\mathrm{R}$ & $<.05$ & $<.05$ & -- & .08 & .05 & $<.05$ & $<.05$ & $<.05$ & $<.05$ & -- & $<.05$ & .05 & $<.05$ & $<.05$ & -- & $<.05$ & $<.05$ & $<.05$ \\
\hline 01/20/99 & 1030 & $\mathrm{~L}$ & $<.05$ & $<.05$ & -- & .07 & .05 & $<.05$ & $<.05$ & $<.05$ & $<.05$ & -- & $<.05$ & .05 & $<.05$ & $<.05$ & -- & $<.05$ & $<.05$ & $<.05$ \\
\hline 02/03/99 & 1000 & $\mathrm{R}$ & $<.05$ & $<.05$ & -- & $<.05$ & $<.05$ & $<.05$ & $<.05$ & $<.05$ & $<.05$ & -- & $<.05$ & $<.05$ & $<.05$ & $<.05$ & -- & $<.05$ & $<.05$ & $<.05$ \\
\hline 02/23/99 & 1000 & $\mathrm{R}$ & $<.05$ & $<.05$ & -- & .13 & .05 & $<.05$ & $<.05$ & $<.05$ & $<.05$ & -- & $<.05$ & $<.05$ & $<.05$ & $<.05$ & -- & $<.05$ & $<.05$ & $<.05$ \\
\hline 02/23/99 & 1000 & $\mathrm{~L}$ & $<.05$ & $<.05$ & -- & $<.05$ & $<.05$ & $<.05$ & $<.05$ & $<.05$ & $<.05$ & -- & $<.05$ & $<.05$ & $<.05$ & $<.05$ & -- & $<.05$ & $<.05$ & $<.05$ \\
\hline
\end{tabular}


Table 4. Results of analysis of selected triazine herbicides and degradation products by gas chromatography/mass spectrometry methods GCS (1993-96) and GCR (1996-2003) at the U.S. Geological Survey Organic Geochemistry Research Laboratory, Lawrence, Kansas, for water samples collected from the Mississippi River at Baton Rouge,

Louisiana, 1993-2003.-Continued

[R, regular sample; L, laboratory duplicate sample; --, no data; <, less than; ESA, ethanesulfonic acid; ELISA, enzyme-linked immunosorbent assay; ametryn, deisopropylprometryn, demethylnorflurazon, flufenacet, norflurazon, pendimethalin, propachlor, propanil, propazine, prometryn, terbutryn, and 3-trifluoromethylaniline were not detected]

\begin{tabular}{|c|c|c|c|c|c|c|c|c|c|c|c|c|c|c|c|c|c|c|c|c|}
\hline \multirow[b]{2}{*}{$\begin{array}{c}\text { Date of } \\
\text { collection } \\
\text { (month/ } \\
\text { day/year) }\end{array}$} & \multirow[b]{2}{*}{$\begin{array}{l}\text { Collec- } \\
\text { tion } \\
\text { time } \\
\text { (24- } \\
\text { hour) }\end{array}$} & \multirow[b]{2}{*}{$\begin{array}{l}\text { Sam- } \\
\text { ple } \\
\text { type }\end{array}$} & \multicolumn{18}{|c|}{ Concentration, in micrograms per liter } \\
\hline & & & $\begin{array}{c}\text { Aceto- } \\
\text { chlor }\end{array}$ & $\begin{array}{l}\text { Ala- } \\
\text { chlor }\end{array}$ & $\begin{array}{l}\text { Ala- } \\
\text { chlor } \\
\text { ESA } \\
\text { ELISA }\end{array}$ & $\begin{array}{l}\text { Atra- } \\
\text { zine }\end{array}$ & $\begin{array}{l}\text { De- } \\
\text { ethyl- } \\
\text { atra- } \\
\text { zine }\end{array}$ & $\begin{array}{l}\text { Deiso- } \\
\text { propyl- } \\
\text { atrazine }\end{array}$ & $\begin{array}{l}\text { Cyan- } \\
\text { azine }\end{array}$ & $\begin{array}{l}\text { Cyan- } \\
\text { azine } \\
\text { amide }\end{array}$ & $\begin{array}{c}\text { De- } \\
\text { methyl- } \\
\text { fluo- } \\
\text { metu- } \\
\text { ron }\end{array}$ & $\begin{array}{l}\text { Di- } \\
\text { meth- } \\
\text { enamid }\end{array}$ & $\begin{array}{l}\text { Fluo- } \\
\text { met- } \\
\text { uron }\end{array}$ & $\begin{array}{l}\text { Meto- } \\
\text { lachlor }\end{array}$ & $\begin{array}{l}\text { Metri- } \\
\text { buzin }\end{array}$ & $\begin{array}{l}\text { Moli- } \\
\text { nate }\end{array}$ & $\begin{array}{l}\text { Pro- } \\
\text { meton }\end{array}$ & $\begin{array}{l}\text { Sima- } \\
\text { zine }\end{array}$ & $\begin{array}{c}\text { Tri- } \\
\text { fluralin }\end{array}$ & $\begin{array}{c}\text { 3-triflu- } \\
\text { oro- } \\
\text { methyl- } \\
\text { phenyl- } \\
\text { urea }\end{array}$ \\
\hline $03 / 12 / 99$ & 0945 & $\mathrm{R}$ & $<0.05$ & $<0.05$ & -- & 0.06 & $<0.05$ & $<0.05$ & $<0.05$ & $<0.05$ & $<0.05$ & $<0.05$ & $<0.05$ & $<0.05$ & $<0.05$ & $<0.05$ & -- & $<0.05$ & $<0.05$ & $<0.05$ \\
\hline 03/26/99 & 1000 & $\mathrm{R}$ & $<.05$ & $<.05$ & -- & .06 & $<.05$ & $<.05$ & $<.05$ & $<.05$ & $<.05$ & $<.05$ & $<.05$ & $<.05$ & $<.05$ & $<.05$ & -- & $<.05$ & $<.05$ & $<.05$ \\
\hline 04/02/99 & 0800 & $\mathrm{R}$ & $<.05$ & $<.05$ & -- & .06 & $<.05$ & $<.05$ & $<.05$ & $<.05$ & $<.05$ & $<.05$ & $<.05$ & $<.05$ & $<.05$ & $<.05$ & -- & $<.05$ & $<.05$ & $<.05$ \\
\hline 04/08/99 & 1300 & $\mathrm{R}$ & $<.05$ & $<.05$ & -- & .13 & $<.05$ & $<.05$ & $<.05$ & $<.05$ & $<.05$ & $<.05$ & $<.05$ & .08 & $<.05$ & $<.05$ & -- & $<.05$ & $<.05$ & $<.05$ \\
\hline 04/15/99 & 1515 & $\mathrm{R}$ & $<.05$ & $<.05$ & -- & .32 & .05 & $<.05$ & $<.05$ & .21 & $<.05$ & $<.05$ & $<.05$ & .15 & $<.05$ & $<.05$ & -- & $<.05$ & $<.05$ & $<.05$ \\
\hline 04/23/99 & 1245 & $\mathrm{R}$ & $<.05$ & $<.05$ & -- & .43 & .05 & $<.05$ & $<.05$ & $<.05$ & $<.05$ & $<.05$ & $<.05$ & .13 & $<.05$ & $<.05$ & -- & .21 & $<.05$ & $<.05$ \\
\hline 04/30/99 & 1500 & $\mathrm{R}$ & .19 & $<.05$ & -- & 1.0 & .06 & $<.05$ & .07 & $<.05$ & $<.05$ & .10 & $<.05$ & .36 & $<.05$ & $<.05$ & -- & .06 & $<.05$ & $<.05$ \\
\hline 05/07/99 & 1000 & $\mathrm{R}$ & .11 & $<.05$ & -- & .90 & .05 & $<.05$ & $<.05$ & $<.05$ & $<.05$ & $<.05$ & $<.05$ & .39 & $<.05$ & $<.05$ & -- & $<.05$ & $<.05$ & $<.05$ \\
\hline 05/17/99 & 1000 & $\mathrm{R}$ & .17 & $<.05$ & -- & 1.2 & .17 & .08 & .07 & $<.05$ & $<.05$ & $<.05$ & .12 & .52 & $<.05$ & $<.05$ & -- & .16 & $<.05$ & $<.05$ \\
\hline 05/25/99 & 1100 & $\mathrm{R}$ & .16 & $<.05$ & -- & 1.4 & .17 & $<.05$ & .06 & $<.05$ & $<.05$ & .06 & .12 & .50 & $<.05$ & $<.05$ & -- & .23 & $<.05$ & $<.05$ \\
\hline 06/03/99 & 1030 & $\mathrm{R}$ & .49 & $<.05$ & -- & .92 & .37 & .17 & .06 & $<.05$ & $<.05$ & .23 & $<.05$ & .67 & $<.05$ & $<.05$ & -- & .06 & $<.05$ & $<.05$ \\
\hline 06/10/99 & 1200 & $\mathrm{R}$ & .47 & $<.05$ & -- & .50 & .16 & .05 & $<.05$ & $<.05$ & $<.05$ & .12 & $<.05$ & 1.0 & $<.05$ & $<.05$ & -- & $<.05$ & $<.05$ & $<.05$ \\
\hline 06/18/99 & 1100 & $\mathrm{R}$ & .18 & $<.05$ & -- & .39 & .18 & .08 & $<.05$ & $<.05$ & $<.05$ & .22 & $<.05$ & .46 & $<.05$ & $<.05$ & -- & $<.05$ & $<.05$ & $<.05$ \\
\hline 06/18/99 & 1100 & $\mathrm{~L}$ & .18 & $<.05$ & -- & .36 & .18 & .08 & $<.05$ & $<.05$ & $<.05$ & .21 & $<.05$ & .36 & $<.05$ & $<.05$ & -- & $<.05$ & $<.05$ & $<.05$ \\
\hline 06/25/99 & 1145 & $\mathrm{R}$ & .37 & .05 & -- & .39 & .38 & .20 & $<.05$ & $<.05$ & $<.05$ & $<.05$ & .06 & .78 & $<.05$ & $<.05$ & -- & $<.05$ & $<.05$ & $<.05$ \\
\hline 06/25/99 & 1145 & $\mathrm{~L}$ & .37 & $<.05$ & -- & .49 & .29 & .09 & $<.05$ & $<.05$ & $<.05$ & $<.05$ & $<.05$ & .84 & $<.05$ & $<.05$ & -- & $<.05$ & $<.05$ & $<.05$ \\
\hline 07/09/99 & 1530 & $\mathrm{R}$ & .08 & $<.05$ & -- & .21 & .22 & .14 & $<.05$ & .22 & $<.05$ & $<.05$ & $<.05$ & .34 & $<.05$ & $<.05$ & -- & $<.05$ & $<.05$ & $<.05$ \\
\hline 08/06/99 & 1045 & $\mathrm{R}$ & $<.05$ & $<.05$ & -- & .52 & .20 & .08 & .08 & .19 & $<.05$ & $<.05$ & $<.05$ & .23 & $<.05$ & $<.05$ & -- & $<.05$ & $<.05$ & $<.05$ \\
\hline 08/17/99 & 1500 & $\mathrm{R}$ & $<.05$ & $<.05$ & -- & .32 & .17 & .07 & $<.05$ & .25 & $<.05$ & $<.05$ & $<.05$ & .13 & $<.05$ & $<.05$ & -- & $<.05$ & $<.05$ & $<.05$ \\
\hline 08/17/99 & 1500 & $\mathrm{~L}$ & $<.05$ & $<.05$ & -- & .31 & .16 & .06 & $<.05$ & .42 & $<.05$ & $<.05$ & $<.05$ & .13 & $<.05$ & $<.05$ & -- & $<.05$ & $<.05$ & $<.05$ \\
\hline
\end{tabular}


Table 4. Results of analysis of selected triazine herbicides and degradation products by gas chromatography/mass spectrometry methods GCS (1993-96) and GCR (1996-2003) at the U.S. Geological Survey Organic Geochemistry Research Laboratory, Lawrence, Kansas, for water samples collected from the Mississippi River at Baton Rouge,

Louisiana, 1993-2003.-Continued

[R, regular sample; L, laboratory duplicate sample; --, no data; <, less than; ESA, ethanesulfonic acid; ELISA, enzyme-linked immunosorbent assay; ametryn, deisopropylprometryn, demethylnorflurazon, flufenacet, norflurazon, pendimethalin, propachlor, propanil, propazine, prometryn, terbutryn, and 3-trifluoromethylaniline were not detected]

\begin{tabular}{|c|c|c|c|c|c|c|c|c|c|c|c|c|c|c|c|c|c|c|c|c|}
\hline \multirow[b]{2}{*}{$\begin{array}{c}\text { Date of } \\
\text { collection } \\
\text { (month/ } \\
\text { day/year) }\end{array}$} & \multirow[b]{2}{*}{$\begin{array}{l}\text { Collec- } \\
\text { tion } \\
\text { time } \\
\text { (24- } \\
\text { hour) }\end{array}$} & \multirow[b]{2}{*}{$\begin{array}{l}\text { Sam- } \\
\text { ple } \\
\text { type }\end{array}$} & \multicolumn{18}{|c|}{ Concentration, in micrograms per liter } \\
\hline & & & $\begin{array}{l}\text { Aceto- } \\
\text { chlor }\end{array}$ & $\begin{array}{l}\text { Ala- } \\
\text { chlor }\end{array}$ & $\begin{array}{l}\text { Ala- } \\
\text { chlor } \\
\text { ESA } \\
\text { ELISA }\end{array}$ & $\begin{array}{l}\text { Atra- } \\
\text { zine }\end{array}$ & $\begin{array}{l}\text { De- } \\
\text { ethyl- } \\
\text { atra- } \\
\text { zine }\end{array}$ & $\begin{array}{l}\text { Deiso- } \\
\text { propyl- } \\
\text { atrazine }\end{array}$ & $\begin{array}{l}\text { Cyan- } \\
\text { azine }\end{array}$ & $\begin{array}{l}\text { Cyan- } \\
\text { azine } \\
\text { amide }\end{array}$ & $\begin{array}{c}\text { De- } \\
\text { methyl- } \\
\text { fluo- } \\
\text { metu- } \\
\text { ron }\end{array}$ & $\begin{array}{c}\text { Di- } \\
\text { meth- } \\
\text { enamid }\end{array}$ & $\begin{array}{l}\text { Fluo- } \\
\text { met- } \\
\text { uron }\end{array}$ & $\begin{array}{l}\text { Meto- } \\
\text { lachlor }\end{array}$ & $\begin{array}{l}\text { Metri- } \\
\text { buzin }\end{array}$ & $\begin{array}{l}\text { Moli- } \\
\text { nate }\end{array}$ & $\begin{array}{l}\text { Pro- } \\
\text { meton }\end{array}$ & $\begin{array}{l}\text { Sima- } \\
\text { zine }\end{array}$ & $\begin{array}{c}\text { Tri- } \\
\text { fluralin }\end{array}$ & $\begin{array}{c}\text { 3-triflu- } \\
\text { oro- } \\
\text { methyl- } \\
\text { phenyl- } \\
\text { urea }\end{array}$ \\
\hline $08 / 23 / 99$ & 1400 & $\mathrm{R}$ & $<0.05$ & $<0.05$ & -- & 0.48 & 0.18 & 0.09 & $<0.05$ & $<0.05$ & $<0.05$ & $<0.05$ & $<0.05$ & 0.16 & $<0.05$ & $<0.05$ & -- & $<0.05$ & $<0.05$ & $<0.05$ \\
\hline 08/23/99 & 1400 & $\mathrm{~L}$ & $<.05$ & $<.05$ & -- & .37 & .14 & .05 & $<.05$ & $<.05$ & $<.05$ & $<.05$ & $<.05$ & .11 & $<.05$ & $<.05$ & -- & $<.05$ & $<.05$ & $<.05$ \\
\hline 09/02/99 & 1445 & $\mathrm{R}$ & $<.05$ & $<.05$ & -- & .15 & .07 & $<.05$ & $<.05$ & $<.05$ & $<.05$ & $<.05$ & $<.05$ & .06 & $<.05$ & $<.05$ & -- & $<.05$ & $<.05$ & $<.05$ \\
\hline 09/27/99 & 1530 & $\mathrm{R}$ & $<.05$ & $<.05$ & -- & .23 & .08 & $<.05$ & $<.05$ & $<.05$ & $<.05$ & $<.05$ & $<.05$ & .05 & $<.05$ & $<.05$ & -- & $<.05$ & $<.05$ & $<.05$ \\
\hline $10 / 04 / 99$ & 1400 & $\mathrm{R}$ & $<.05$ & $<.05$ & -- & .48 & .13 & $<.05$ & $<.05$ & $<.05$ & $<.05$ & $<.05$ & $<.05$ & .09 & $<.05$ & $<.05$ & -- & $<.05$ & $<.05$ & $<.05$ \\
\hline $10 / 20 / 99$ & 1430 & $\mathrm{R}$ & $<.05$ & $<.05$ & -- & .13 & .05 & $<.05$ & $<.05$ & $<.05$ & $<.05$ & $<.05$ & $<.05$ & $<.05$ & $<.05$ & $<.05$ & -- & $<.05$ & .06 & $<.05$ \\
\hline $10 / 20 / 99$ & 1430 & $\mathrm{~L}$ & $<.05$ & $<.05$ & -- & .14 & .05 & $<.05$ & $<.05$ & $<.05$ & $<.05$ & $<.05$ & $<.05$ & $<.05$ & $<.05$ & $<.05$ & -- & $<.05$ & $<.05$ & $<.05$ \\
\hline $11 / 01 / 99$ & 1430 & $\mathrm{R}$ & $<.05$ & $<.05$ & -- & .09 & $<.05$ & $<.05$ & $<.05$ & .79 & $<.05$ & $<.05$ & $<.05$ & $<.05$ & $<.05$ & $<.05$ & -- & $<.05$ & $<.05$ & $<.05$ \\
\hline $11 / 19 / 99$ & 1700 & $\mathrm{R}$ & $<.05$ & $<.05$ & -- & .09 & $<.05$ & $<.05$ & $<.05$ & $<.05$ & $<.05$ & $<.05$ & $<.05$ & $<.05$ & $<.05$ & $<.05$ & -- & $<.05$ & $<.05$ & $<.05$ \\
\hline $12 / 06 / 99$ & 1330 & $\mathrm{R}$ & $<.05$ & $<.05$ & -- & .07 & $<.05$ & $<.05$ & $<.05$ & $<.05$ & $<.05$ & $<.05$ & $<.05$ & $<.05$ & $<.05$ & $<.05$ & -- & $<.05$ & $<.05$ & $<.05$ \\
\hline $01 / 13 / 00$ & 1500 & $\mathrm{R}$ & $<.05$ & $<.05$ & -- & .08 & $<.05$ & $<.05$ & $<.05$ & $<.05$ & $<.05$ & $<.05$ & $<.05$ & $<.05$ & $<.05$ & $<.05$ & -- & $<.05$ & $<.05$ & $<.05$ \\
\hline $02 / 14 / 00$ & 1545 & $\mathrm{R}$ & $<.05$ & $<.05$ & -- & .08 & $<.05$ & $<.05$ & $<.05$ & .44 & $<.05$ & $<.05$ & $<.05$ & $<.05$ & $<.05$ & $<.05$ & -- & $<.05$ & $<.05$ & $<.05$ \\
\hline $05 / 12 / 00$ & 1500 & $\mathrm{R}$ & $<.05$ & $<.05$ & -- & 1.1 & .15 & $<.05$ & $<.05$ & $<.05$ & $<.05$ & $<.05$ & .16 & .36 & $<.05$ & $<.05$ & -- & .12 & $<.05$ & $<.05$ \\
\hline $05 / 23 / 00$ & 1300 & $\mathrm{R}$ & $<.05$ & $<.05$ & -- & 1.1 & .10 & $<.05$ & $<.05$ & $<.05$ & $<.05$ & $<.05$ & .15 & .20 & $<.05$ & $<.05$ & -- & .08 & $<.05$ & $<.05$ \\
\hline 06/20/00 & 1000 & $\mathrm{R}$ & .12 & $<.05$ & -- & 1.2 & .22 & .11 & $<.05$ & $<.05$ & $<.05$ & .05 & $<.05$ & .31 & $<.05$ & $<.05$ & -- & .05 & $<.05$ & $<.05$ \\
\hline 06/29/00 & 1145 & $\mathrm{R}$ & .05 & $<.05$ & -- & .77 & .20 & .08 & $<.05$ & $<.05$ & $<.05$ & $<.05$ & $<.05$ & .17 & $<.05$ & $<.05$ & -- & $<.05$ & $<.05$ & $<.05$ \\
\hline $06 / 29 / 00$ & 1145 & $\mathrm{~L}$ & .05 & $<.05$ & -- & .76 & .18 & .08 & $<.05$ & $<.05$ & $<.05$ & $<.05$ & $<.05$ & .16 & $<.05$ & $<.05$ & -- & $<.05$ & $<.05$ & $<.05$ \\
\hline 07/07/00 & 1400 & $\mathrm{R}$ & .05 & $<.05$ & -- & 1.1 & .40 & .19 & $<.05$ & $<.05$ & $<.05$ & $<.05$ & $<.05$ & .23 & $<.05$ & $<.05$ & -- & $<.05$ & $<.05$ & $<.05$ \\
\hline 07/18/00 & 1200 & $\mathrm{R}$ & $<.05$ & $<.05$ & -- & .69 & .21 & .10 & $<.05$ & $<.05$ & $<.05$ & $<.05$ & $<.05$ & $<.05$ & $<.05$ & $<.05$ & -- & $<.05$ & $<.05$ & $<.05$ \\
\hline 08/03/00 & 1125 & $\mathrm{R}$ & $<.05$ & $<.05$ & -- & .42 & .15 & $<.05$ & $<.05$ & $<.05$ & $<.05$ & $<.05$ & $<.05$ & .08 & $<.05$ & $<.05$ & -- & $<.05$ & $<.05$ & $<.05$ \\
\hline
\end{tabular}


Table 4. Results of analysis of selected triazine herbicides and degradation products by gas chromatography/mass spectrometry methods GCS (1993-96) and GCR (1996-2003) at the U.S. Geological Survey Organic Geochemistry Research Laboratory, Lawrence, Kansas, for water samples collected from the Mississippi River at Baton Rouge,

Louisiana, 1993-2003.-Continued

[R, regular sample; L, laboratory duplicate sample; --, no data; <, less than; ESA, ethanesulfonic acid; ELISA, enzyme-linked immunosorbent assay; ametryn, deisopropylprometryn, demethylnorflurazon, flufenacet, norflurazon, pendimethalin, propachlor, propanil, propazine, prometryn, terbutryn, and 3-trifluoromethylaniline were not detected]

\begin{tabular}{|c|c|c|c|c|c|c|c|c|c|c|c|c|c|c|c|c|c|c|c|c|}
\hline \multirow[b]{2}{*}{$\begin{array}{c}\text { Date of } \\
\text { collection } \\
\text { (month/ } \\
\text { day/year) }\end{array}$} & \multirow[b]{2}{*}{$\begin{array}{l}\text { Collec- } \\
\text { tion } \\
\text { time } \\
(24- \\
\text { hour) }\end{array}$} & \multirow[b]{2}{*}{$\begin{array}{l}\text { Sam- } \\
\text { ple } \\
\text { type }\end{array}$} & \multicolumn{18}{|c|}{ Concentration, in micrograms per liter } \\
\hline & & & $\begin{array}{l}\text { Aceto- } \\
\text { chlor }\end{array}$ & $\begin{array}{l}\text { Ala- } \\
\text { chlor }\end{array}$ & $\begin{array}{l}\text { Ala- } \\
\text { chlor } \\
\text { ESA } \\
\text { ELISA }\end{array}$ & $\begin{array}{l}\text { Atra- } \\
\text { zine }\end{array}$ & $\begin{array}{l}\text { De- } \\
\text { ethyl- } \\
\text { atra- } \\
\text { zine }\end{array}$ & $\begin{array}{l}\text { Deiso- } \\
\text { propyl- } \\
\text { atrazine }\end{array}$ & $\begin{array}{l}\text { Cyan- } \\
\text { azine }\end{array}$ & $\begin{array}{l}\text { Cyan- } \\
\text { azine } \\
\text { amide }\end{array}$ & $\begin{array}{c}\text { De- } \\
\text { methyl- } \\
\text { fluo- } \\
\text { metu- } \\
\text { ron }\end{array}$ & $\begin{array}{l}\text { Di- } \\
\text { meth- } \\
\text { enamid }\end{array}$ & $\begin{array}{l}\text { Fluo- } \\
\text { met- } \\
\text { uron }\end{array}$ & $\begin{array}{l}\text { Meto- } \\
\text { lachlor }\end{array}$ & $\begin{array}{l}\text { Metri- } \\
\text { buzin }\end{array}$ & $\begin{array}{l}\text { Moli- } \\
\text { nate }\end{array}$ & $\begin{array}{l}\text { Pro- } \\
\text { meton }\end{array}$ & $\begin{array}{c}\text { Sima- } \\
\text { zine }\end{array}$ & $\begin{array}{c}\text { Tri- } \\
\text { fluralin }\end{array}$ & $\begin{array}{c}\text { 3-triflu- } \\
\text { oro- } \\
\text { methyl- } \\
\text { phenyl- } \\
\text { urea }\end{array}$ \\
\hline $08 / 11 / 00$ & 1045 & $\mathrm{R}$ & $<0.05$ & $<0.05$ & -- & 0.44 & 0.14 & 0.05 & $<0.05$ & $<0.05$ & $<0.05$ & $<0.05$ & $<0.05$ & $<0.05$ & $<0.05$ & $<0.05$ & -- & $<0.05$ & $<0.05$ & $<0.05$ \\
\hline $08 / 21 / 00$ & 1100 & $\mathrm{R}$ & $<.05$ & $<.05$ & -- & .30 & .09 & $<.05$ & $<.05$ & $<.05$ & $<.05$ & $<.05$ & $<.05$ & $<.05$ & $<.05$ & $<.05$ & -- & $<.05$ & $<.05$ & $<.05$ \\
\hline 08/29/00 & 1230 & $\mathrm{R}$ & $<.05$ & $<.05$ & -- & .29 & .09 & $<.05$ & $<.05$ & $<.05$ & $<.05$ & $<.05$ & $<.05$ & $<.05$ & $<.05$ & $<.05$ & -- & $<.05$ & $<.05$ & $<.05$ \\
\hline 09/11/00 & 1045 & $\mathrm{R}$ & $<.05$ & $<.05$ & -- & .19 & .08 & $<.05$ & $<.05$ & $<.05$ & $<.05$ & $<.05$ & $<.05$ & $<.05$ & $<.05$ & $<.05$ & -- & $<.05$ & $<.05$ & $<.05$ \\
\hline 09/11/00 & 1045 & $\mathrm{~L}$ & $<.05$ & $<.05$ & -- & .20 & .07 & $<.05$ & $<.05$ & $<.05$ & $<.05$ & $<.05$ & $<.05$ & $<.05$ & $<.05$ & $<.05$ & -- & $<.05$ & $<.05$ & $<.05$ \\
\hline 09/27/00 & 1400 & $\mathrm{R}$ & $<.05$ & $<.05$ & -- & .09 & .05 & $<.05$ & $<.05$ & $<.05$ & $<.05$ & $<.05$ & $<.05$ & $<.05$ & $<.05$ & $<.05$ & -- & $<.05$ & $<.05$ & $<.05$ \\
\hline 09/27/00 & 1400 & $\mathrm{~L}$ & $<.05$ & $<.05$ & -- & .10 & .05 & $<.05$ & $<.05$ & $<.05$ & $<.05$ & $<.05$ & $<.05$ & $<.05$ & $<.05$ & $<.05$ & -- & $<.05$ & $<.05$ & $<.05$ \\
\hline $10 / 10 / 00$ & 1130 & $\mathrm{R}$ & $<.05$ & $<.05$ & -- & .07 & $<.05$ & $<.05$ & $<.05$ & $<.05$ & $<.05$ & $<.05$ & $<.05$ & $<.05$ & $<.05$ & $<.05$ & -- & $<.05$ & $<.05$ & $<.05$ \\
\hline $10 / 19 / 00$ & 1145 & $\mathrm{R}$ & $<.05$ & $<.05$ & -- & .13 & $<.05$ & $<.05$ & $<.05$ & $<.05$ & $<.05$ & $<.05$ & $<.05$ & $<.05$ & $<.05$ & $<.05$ & -- & $<.05$ & $<.05$ & $<.05$ \\
\hline $11 / 06 / 00$ & 1400 & $\mathrm{R}$ & $<.05$ & $<.05$ & -- & .17 & $<.05$ & $<.05$ & $<.05$ & $<.05$ & $<.05$ & $<.05$ & $<.05$ & $<.05$ & $<.05$ & $<.05$ & -- & $<.05$ & $<.05$ & $<.05$ \\
\hline $11 / 20 / 00$ & 1230 & $\mathrm{R}$ & $<.05$ & $<.05$ & -- & .14 & $<.05$ & $<.05$ & $<.05$ & $<.05$ & $<.05$ & $<.05$ & $<.05$ & $<.05$ & $<.05$ & $<.05$ & -- & $<.05$ & $<.05$ & $<.05$ \\
\hline $12 / 04 / 00$ & 1330 & $\mathrm{R}$ & $<.05$ & $<.05$ & -- & .12 & $<.05$ & $<.05$ & $<.05$ & $<.05$ & $<.05$ & $<.05$ & $<.05$ & $<.05$ & $<.05$ & $<.05$ & -- & .17 & $<.05$ & $<.05$ \\
\hline $12 / 18 / 00$ & 1130 & $\mathrm{R}$ & $<.05$ & $<.05$ & -- & .08 & $<.05$ & $<.05$ & $<.05$ & $<.05$ & $<.05$ & $<.05$ & $<.05$ & $<.05$ & $<.05$ & $<.05$ & -- & .07 & $<.05$ & $<.05$ \\
\hline 01/05/01 & 1000 & $\mathrm{R}$ & $<.05$ & $<.05$ & -- & .05 & $<.05$ & $<.05$ & $<.05$ & $<.05$ & $<.05$ & $<.05$ & $<.05$ & $<.05$ & $<.05$ & $<.05$ & -- & $<.05$ & $<.05$ & $<.05$ \\
\hline 01/05/01 & 1000 & $\mathrm{~L}$ & $<.05$ & $<.05$ & -- & .05 & $<.05$ & $<.05$ & $<.05$ & $<.05$ & $<.05$ & $<.05$ & $<.05$ & $<.05$ & $<.05$ & $<.05$ & -- & $<.05$ & $<.05$ & $<.05$ \\
\hline 02/08/01 & 1130 & $\mathrm{R}$ & $<.05$ & $<.05$ & -- & .08 & $<.05$ & $<.05$ & $<.05$ & $<.05$ & $<.05$ & $<.05$ & $<.05$ & $<.05$ & $<.05$ & $<.05$ & -- & .05 & $<.05$ & $<.05$ \\
\hline $02 / 23 / 01$ & 1400 & $\mathrm{R}$ & $<.05$ & $<.05$ & -- & .10 & $<.05$ & $<.05$ & $<.05$ & $<.05$ & $<.05$ & $<.05$ & $<.05$ & $<.05$ & $<.05$ & $<.05$ & -- & .11 & $<.05$ & $<.05$ \\
\hline 03/06/01 & 1100 & $\mathrm{R}$ & $<.05$ & $<.05$ & -- & .06 & $<.05$ & $<.05$ & $<.05$ & $<.05$ & $<.05$ & $<.05$ & $<.05$ & $<.05$ & $<.05$ & $<.05$ & -- & $<.05$ & $<.05$ & $<.05$ \\
\hline $03 / 23 / 01$ & 1500 & $\mathrm{R}$ & $<.05$ & $<.05$ & -- & .10 & $<.05$ & $<.05$ & $<.05$ & $<.05$ & $<.05$ & $<.05$ & $<.05$ & .06 & $<.05$ & $<.05$ & -- & $<.05$ & $<.05$ & $<.05$ \\
\hline 04/06/01 & 1430 & $\mathrm{R}$ & $<.05$ & $<.05$ & -- & .07 & $<.05$ & $<.05$ & $<.05$ & $<.05$ & $<.05$ & $<.05$ & $<.05$ & .05 & $<.05$ & $<.05$ & -- & $<.05$ & $<.05$ & $<.05$ \\
\hline
\end{tabular}


Table 4. Results of analysis of selected triazine herbicides and degradation products by gas chromatography/mass spectrometry methods GCS (1993-96) and GCR (1996-2003) at the U.S. Geological Survey Organic Geochemistry Research Laboratory, Lawrence, Kansas, for water samples collected from the Mississippi River at Baton Rouge,

Louisiana, 1993-2003.-Continued

[R, regular sample; L, laboratory duplicate sample; --, no data; <, less than; ESA, ethanesulfonic acid; ELISA, enzyme-linked immunosorbent assay; ametryn, deisopropylprometryn, demethylnorflurazon, flufenacet, norflurazon, pendimethalin, propachlor, propanil, propazine, prometryn, terbutryn, and 3-trifluoromethylaniline were not detected]

\begin{tabular}{|c|c|c|c|c|c|c|c|c|c|c|c|c|c|c|c|c|c|c|c|c|}
\hline \multirow[b]{2}{*}{$\begin{array}{c}\text { Date of } \\
\text { collection } \\
\text { (month/ } \\
\text { day/year) }\end{array}$} & \multirow[b]{2}{*}{$\begin{array}{l}\text { Collec- } \\
\text { tion } \\
\text { time } \\
\text { (24- } \\
\text { hour) }\end{array}$} & \multirow[b]{2}{*}{$\begin{array}{l}\text { Sam- } \\
\text { ple } \\
\text { type }\end{array}$} & \multicolumn{18}{|c|}{ Concentration, in micrograms per liter } \\
\hline & & & $\begin{array}{l}\text { Aceto- } \\
\text { chlor }\end{array}$ & $\begin{array}{l}\text { Ala- } \\
\text { chlor }\end{array}$ & $\begin{array}{l}\text { Ala- } \\
\text { chlor } \\
\text { ESA } \\
\text { ELISA }\end{array}$ & $\begin{array}{l}\text { Atra- } \\
\text { zine }\end{array}$ & $\begin{array}{l}\text { De- } \\
\text { ethyl- } \\
\text { atra- } \\
\text { zine }\end{array}$ & $\begin{array}{l}\text { Deiso- } \\
\text { propyl- } \\
\text { atrazine }\end{array}$ & $\begin{array}{l}\text { Cyan- } \\
\text { azine }\end{array}$ & $\begin{array}{l}\text { Cyan- } \\
\text { azine } \\
\text { amide }\end{array}$ & $\begin{array}{l}\text { De- } \\
\text { methyl- } \\
\text { fluo- } \\
\text { metu- } \\
\text { ron }\end{array}$ & $\begin{array}{c}\text { Di- } \\
\text { meth- } \\
\text { enamid }\end{array}$ & $\begin{array}{l}\text { Fluo- } \\
\text { met- } \\
\text { uron }\end{array}$ & $\begin{array}{l}\text { Meto- } \\
\text { lachlor }\end{array}$ & $\begin{array}{l}\text { Metri- } \\
\text { buzin }\end{array}$ & $\begin{array}{l}\text { Moli- } \\
\text { nate }\end{array}$ & $\begin{array}{l}\text { Pro- } \\
\text { meton }\end{array}$ & $\begin{array}{l}\text { Sima- } \\
\text { zine }\end{array}$ & $\begin{array}{c}\text { Tri- } \\
\text { fluralin }\end{array}$ & $\begin{array}{c}\text { 3-triflu- } \\
\text { oro- } \\
\text { methyl- } \\
\text { phenyl- } \\
\text { urea }\end{array}$ \\
\hline $05 / 03 / 01$ & 1200 & $\mathrm{R}$ & $<0.05$ & $<0.05$ & -- & 0.38 & 0.06 & $<0.05$ & $<0.05$ & $<0.05$ & $<0.05$ & $<0.05$ & $<0.05$ & 0.20 & $<0.05$ & $<0.05$ & -- & $<0.05$ & $<0.05$ & $<0.05$ \\
\hline $05 / 17 / 01$ & 1415 & $\mathrm{R}$ & $<.05$ & $<.05$ & -- & .21 & .06 & $<.05$ & $<.05$ & $<.05$ & $<.05$ & $<.05$ & $<.05$ & .18 & $<.05$ & $<.05$ & -- & $<.05$ & $<.05$ & $<.05$ \\
\hline $05 / 24 / 01$ & 1400 & $\mathrm{R}$ & .31 & $<.05$ & -- & .74 & .17 & .09 & $<.05$ & $<.05$ & $<.05$ & .07 & $<.05$ & .47 & $<.05$ & $<.05$ & -- & $<.05$ & $<.05$ & $<.05$ \\
\hline $05 / 24 / 01$ & 1400 & $\mathrm{~L}$ & .32 & $<.05$ & -- & .76 & .18 & .09 & $<.05$ & $<.05$ & $<.05$ & .07 & $<.05$ & .48 & $<.05$ & $<.05$ & -- & .05 & $<.05$ & $<.05$ \\
\hline 05/31/01 & 1400 & $\mathrm{R}$ & .19 & $<.05$ & -- & .61 & .19 & .08 & $<.05$ & $<.05$ & $<.05$ & .07 & $<.05$ & .41 & $<.05$ & $<.05$ & -- & .05 & $<.05$ & $<.05$ \\
\hline 06/08/01 & 1400 & $\mathrm{R}$ & .16 & $<.05$ & -- & .50 & .22 & .10 & $<.05$ & $<.05$ & $<.05$ & $<.05$ & $<.05$ & .35 & $<.05$ & $<.05$ & -- & .08 & $<.05$ & $<.05$ \\
\hline 07/02/01 & 1200 & $\mathrm{R}$ & .14 & $<.05$ & -- & .75 & .48 & .08 & $<.05$ & $<.05$ & $<.05$ & .06 & $<.05$ & .40 & $<.05$ & $<.05$ & -- & .05 & $<.05$ & $<.05$ \\
\hline 07/16/01 & 1330 & $\mathrm{R}$ & $<.05$ & $<.05$ & -- & .77 & .16 & $<.05$ & $<.05$ & $<.05$ & $<.05$ & $<.05$ & $<.05$ & .20 & $<.05$ & $<.05$ & -- & $<.05$ & $<.05$ & $<.05$ \\
\hline 07/30/01 & 1430 & $\mathrm{R}$ & $<.05$ & $<.05$ & -- & .41 & .13 & .08 & $<.05$ & $<.05$ & $<.05$ & $<.05$ & $<.05$ & .11 & $<.05$ & $<.05$ & -- & $<.05$ & $<.05$ & $<.05$ \\
\hline 07/30/01 & 1430 & $\mathrm{~L}$ & $<.05$ & $<.05$ & -- & .39 & .13 & .09 & $<.05$ & $<.05$ & $<.05$ & $<.05$ & $<.05$ & .10 & $<.05$ & $<.05$ & -- & $<.05$ & $<.05$ & $<.05$ \\
\hline 08/13/01 & 1130 & $\mathrm{R}$ & $<.05$ & $<.05$ & -- & .42 & .15 & .08 & $<.05$ & $<.05$ & $<.05$ & $<.05$ & $<.05$ & .07 & $<.05$ & $<.05$ & -- & $<.05$ & $<.05$ & $<.05$ \\
\hline 08/28/01 & 1330 & $\mathrm{R}$ & $<.05$ & $<.05$ & -- & .26 & .09 & $<.05$ & $<.05$ & $<.05$ & .18 & $<.05$ & $<.05$ & $<.05$ & $<.05$ & $<.05$ & -- & .12 & $<.05$ & $<.05$ \\
\hline 09/18/01 & 1100 & $\mathrm{R}$ & $<.05$ & $<.05$ & -- & .16 & .06 & $<.05$ & $<.05$ & $<.05$ & .19 & $<.05$ & $<.05$ & $<.05$ & $<.05$ & $<.05$ & -- & .12 & $<.05$ & $<.05$ \\
\hline $10 / 01 / 01$ & 1330 & $\mathrm{R}$ & $<.05$ & $<.05$ & -- & .15 & $<.05$ & $<.05$ & $<.05$ & $<.05$ & $<.05$ & $<.05$ & $<.05$ & $<.05$ & $<.05$ & $<.05$ & -- & $<.05$ & $<.05$ & $<.05$ \\
\hline $10 / 16 / 01$ & 1200 & $\mathrm{R}$ & $<.05$ & $<.05$ & -- & .15 & .05 & $<.05$ & $<.05$ & $<.05$ & $<.05$ & $<.05$ & $<.05$ & $<.05$ & $<.05$ & $<.05$ & -- & $<.05$ & $<.05$ & $<.05$ \\
\hline $10 / 16 / 01$ & 1200 & $\mathrm{~L}$ & $<.05$ & $<.05$ & -- & .14 & .05 & $<.05$ & $<.05$ & $<.05$ & $<.05$ & $<.05$ & $<.05$ & $<.05$ & $<.05$ & $<.05$ & -- & $<.05$ & $<.05$ & $<.05$ \\
\hline 10/31/01 & 1300 & $\mathrm{R}$ & $<.05$ & $<.05$ & -- & .13 & $<.05$ & $<.05$ & $<.05$ & $<.05$ & $<.05$ & $<.05$ & $<.05$ & $<.05$ & $<.05$ & $<.05$ & -- & $<.05$ & $<.05$ & $<.05$ \\
\hline $11 / 14 / 01$ & 1300 & $\mathrm{R}$ & $<.05$ & $<.05$ & -- & .10 & .05 & $<.05$ & $<.05$ & $<.05$ & $<.05$ & $<.05$ & $<.05$ & $<.05$ & $<.05$ & $<.05$ & -- & $<.05$ & $<.05$ & $<.05$ \\
\hline $11 / 14 / 01$ & 1300 & $\mathrm{~L}$ & $<.05$ & $<.05$ & -- & .10 & .06 & $<.05$ & $<.05$ & $<.05$ & $<.05$ & $<.05$ & $<.05$ & $<.05$ & $<.05$ & $<.05$ & -- & $<.05$ & $<.05$ & $<.05$ \\
\hline $11 / 28 / 01$ & 1200 & $\mathrm{R}$ & $<.05$ & $<.05$ & -- & .11 & .06 & $<.05$ & $<.05$ & $<.05$ & $<.05$ & $<.05$ & $<.05$ & $<.05$ & $<.05$ & $<.05$ & -- & $<.05$ & $<.05$ & $<.05$ \\
\hline
\end{tabular}


Table 4. Results of analysis of selected triazine herbicides and degradation products by gas chromatography/mass spectrometry methods GCS (1993-96) and GCR (1996-2003) at the U.S. Geological Survey Organic Geochemistry Research Laboratory, Lawrence, Kansas, for water samples collected from the Mississippi River at Baton Rouge,

Louisiana, 1993-2003.-Continued

[R, regular sample; L, laboratory duplicate sample; --, no data; <, less than; ESA, ethanesulfonic acid; ELISA, enzyme-linked immunosorbent assay; ametryn, deisopropylprometryn, demethylnorflurazon, flufenacet, norflurazon, pendimethalin, propachlor, propanil, propazine, prometryn, terbutryn, and 3-trifluoromethylaniline were not detected]

\begin{tabular}{|c|c|c|c|c|c|c|c|c|c|c|c|c|c|c|c|c|c|c|c|c|}
\hline \multirow[b]{2}{*}{$\begin{array}{c}\text { Date of } \\
\text { collection } \\
\text { (month/ } \\
\text { day/year) }\end{array}$} & \multirow[b]{2}{*}{$\begin{array}{l}\text { Collec- } \\
\text { tion } \\
\text { time } \\
\text { (24- } \\
\text { hour) }\end{array}$} & \multirow[b]{2}{*}{$\begin{array}{l}\text { Sam- } \\
\text { ple } \\
\text { type }\end{array}$} & \multicolumn{18}{|c|}{ Concentration, in micrograms per liter } \\
\hline & & & $\begin{array}{c}\text { Aceto- } \\
\text { chlor }\end{array}$ & $\begin{array}{l}\text { Ala- } \\
\text { chlor }\end{array}$ & $\begin{array}{l}\text { Ala- } \\
\text { chlor } \\
\text { ESA } \\
\text { ELISA }\end{array}$ & $\begin{array}{l}\text { Atra- } \\
\text { zine }\end{array}$ & $\begin{array}{l}\text { De- } \\
\text { ethyl- } \\
\text { atra- } \\
\text { zine }\end{array}$ & $\begin{array}{l}\text { Deiso- } \\
\text { propyl- } \\
\text { atrazine }\end{array}$ & $\begin{array}{l}\text { Cyan- } \\
\text { azine }\end{array}$ & $\begin{array}{l}\text { Cyan- } \\
\text { azine } \\
\text { amide }\end{array}$ & $\begin{array}{c}\text { De- } \\
\text { methyl- } \\
\text { fluo- } \\
\text { metu- } \\
\text { ron }\end{array}$ & $\begin{array}{c}\text { Di- } \\
\text { meth- } \\
\text { enamid }\end{array}$ & $\begin{array}{l}\text { Fluo- } \\
\text { met- } \\
\text { uron }\end{array}$ & $\begin{array}{l}\text { Meto- } \\
\text { lachlor }\end{array}$ & $\begin{array}{l}\text { Metri- } \\
\text { buzin }\end{array}$ & $\begin{array}{l}\text { Moli- } \\
\text { nate }\end{array}$ & $\begin{array}{l}\text { Pro- } \\
\text { meton }\end{array}$ & $\begin{array}{l}\text { Sima- } \\
\text { zine }\end{array}$ & $\begin{array}{c}\text { Tri- } \\
\text { fluralin }\end{array}$ & $\begin{array}{c}\text { 3-triflu- } \\
\text { oro- } \\
\text { methyl- } \\
\text { phenyl- } \\
\text { urea }\end{array}$ \\
\hline$\overline{12 / 10 / 01}$ & 1300 & $\mathrm{R}$ & $<0.05$ & $<0.05$ & -- & 0.12 & 0.05 & 0.05 & $<0.05$ & $<0.05$ & $<0.05$ & $<0.05$ & $<0.05$ & $<0.05$ & $<0.05$ & $<0.05$ & -- & 0.95 & $<0.05$ & $<0.05$ \\
\hline $01 / 03 / 02$ & 1140 & $\mathrm{R}$ & $<.05$ & $<.05$ & -- & .07 & $<.05$ & .05 & $<.05$ & $<.05$ & $<.05$ & $<.05$ & $<.05$ & $<.05$ & $<.05$ & $<.05$ & -- & .31 & $<.05$ & $<.05$ \\
\hline 01/03/02 & 1140 & $\mathrm{~L}$ & $<.05$ & $<.05$ & -- & .07 & $<.05$ & .05 & $<.05$ & $<.05$ & $<.05$ & $<.05$ & $<.05$ & $<.05$ & $<.05$ & $<.05$ & -- & .27 & $<.05$ & $<.05$ \\
\hline $01 / 17 / 02$ & 1100 & $\mathrm{R}$ & $<.05$ & $<.05$ & -- & .07 & $<.05$ & $<.05$ & $<.05$ & $<.05$ & $<.05$ & $<.05$ & $<.05$ & $<.05$ & $<.05$ & $<.05$ & -- & .17 & $<.05$ & $<.05$ \\
\hline $02 / 04 / 02$ & 1300 & $\mathrm{R}$ & $<.05$ & $<.05$ & -- & .05 & $<.05$ & $<.05$ & $<.05$ & $<.05$ & $<.05$ & $<.05$ & $<.05$ & $<.05$ & $<.05$ & $<.05$ & -- & .08 & $<.05$ & $<.05$ \\
\hline $02 / 28 / 02$ & 1230 & $\mathrm{R}$ & $<.05$ & $<.05$ & -- & .10 & $<.05$ & $<.05$ & $<.05$ & $<.05$ & $<.05$ & $<.05$ & $<.05$ & $<.05$ & $<.05$ & $<.05$ & -- & .11 & $<.05$ & $<.05$ \\
\hline $02 / 28 / 02$ & 1230 & $\mathrm{~L}$ & $<.05$ & $<.05$ & -- & .10 & $<.05$ & $<.05$ & $<.05$ & $<.05$ & $<.05$ & $<.05$ & $<.05$ & $<.05$ & $<.05$ & $<.05$ & -- & .11 & $<.05$ & $<.05$ \\
\hline $03 / 11 / 02$ & 1300 & $\mathrm{R}$ & $<.05$ & $<.05$ & -- & .08 & $<.05$ & $<.05$ & $<.05$ & $<.05$ & $<.05$ & $<.05$ & $<.05$ & $<.05$ & $<.05$ & $<.05$ & -- & .07 & $<.05$ & $<.05$ \\
\hline $03 / 28 / 02$ & 1300 & $\mathrm{R}$ & $<.05$ & $<.05$ & -- & .13 & $<.05$ & $<.05$ & $<.05$ & $<.05$ & $<.05$ & $<.05$ & $<.05$ & $<.05$ & $<.05$ & $<.05$ & -- & .10 & $<.05$ & $<.05$ \\
\hline $03 / 28 / 02$ & 1300 & $\mathrm{~L}$ & $<.05$ & $<.05$ & -- & .13 & $<.05$ & $<.05$ & $<.05$ & $<.05$ & $<.05$ & $<.05$ & $<.05$ & $<.05$ & $<.05$ & $<.05$ & -- & .10 & $<.05$ & $<.05$ \\
\hline $04 / 12 / 02$ & 1115 & $\mathrm{R}$ & $<.05$ & $<.05$ & -- & .13 & $<.05$ & $<.05$ & $<.05$ & $<.05$ & $<.05$ & $<.05$ & $<.05$ & $<.05$ & $<.05$ & $<.05$ & -- & .07 & $<.05$ & $<.05$ \\
\hline $04 / 12 / 02$ & 1115 & $\mathrm{~L}$ & $<.05$ & $<.05$ & -- & .14 & $<.05$ & $<.05$ & $<.05$ & $<.05$ & $<.05$ & $<.05$ & $<.05$ & $<.05$ & $<.05$ & $<.05$ & -- & .07 & $<.05$ & $<.05$ \\
\hline $05 / 02 / 02$ & 1300 & $\mathrm{R}$ & $<.05$ & $<.05$ & -- & .54 & .05 & $<.05$ & $<.05$ & $<.05$ & $<.05$ & $<.05$ & $<.05$ & $<.05$ & $<.05$ & $<.05$ & -- & .16 & $<.05$ & $<.05$ \\
\hline $05 / 15 / 02$ & 1300 & $\mathrm{R}$ & .17 & $<.05$ & -- & 2.1 & .15 & .09 & $<.05$ & $<.05$ & .17 & .10 & $<.05$ & .37 & $<.05$ & $<.05$ & -- & .24 & $<.05$ & $<.05$ \\
\hline $05 / 15 / 02$ & 1300 & $\mathrm{~L}$ & .17 & $<.05$ & -- & 2.3 & .15 & .09 & $<.05$ & $<.05$ & .16 & .09 & $<.05$ & .38 & $<.05$ & $<.05$ & -- & .24 & $<.05$ & $<.05$ \\
\hline 06/05/02 & 1200 & $\mathrm{R}$ & .22 & $<.05$ & -- & 1.9 & .27 & .16 & $<.05$ & $<.05$ & $<.05$ & .12 & $<.05$ & .44 & $<.05$ & $<.05$ & -- & .18 & $<.05$ & $<.05$ \\
\hline 06/05/02 & 1200 & $\mathrm{~L}$ & .22 & $<.05$ & -- & 1.8 & .28 & .17 & $<.05$ & $<.05$ & $<.05$ & .10 & $<.05$ & .43 & $<.05$ & $<.05$ & -- & .17 & $<.05$ & $<.05$ \\
\hline $06 / 11 / 02$ & 1030 & $\mathrm{R}$ & .18 & $<.05$ & -- & 1.4 & .20 & .17 & $<.05$ & $<.05$ & $<.05$ & .07 & $<.05$ & .43 & $<.05$ & $<.05$ & -- & .14 & $<.05$ & $<.05$ \\
\hline $07 / 02 / 02$ & 1130 & $\mathrm{R}$ & .27 & $<.05$ & -- & .41 & .35 & .33 & $<.05$ & $<.05$ & $<.05$ & .11 & $<.05$ & .66 & $<.05$ & $<.05$ & -- & $<.05$ & $<.05$ & $<.05$ \\
\hline $07 / 02 / 02$ & 1130 & $\mathrm{~L}$ & .26 & $<.05$ & -- & .38 & .34 & .33 & $<.05$ & $<.05$ & $<.05$ & .10 & $<.05$ & .60 & $<.05$ & $<.05$ & -- & $<.05$ & $<.05$ & $<.05$ \\
\hline
\end{tabular}


Table 4. Results of analysis of selected triazine herbicides and degradation products by gas chromatography/mass spectrometry methods GCS (1993-96) and GCR (1996-2003) at the U.S. Geological Survey Organic Geochemistry Research Laboratory, Lawrence, Kansas, for water samples collected from the Mississippi River at Baton Rouge,

Louisiana, 1993-2003.-Continued

[R, regular sample; L, laboratory duplicate sample; --, no data; <, less than; ESA, ethanesulfonic acid; ELISA, enzyme-linked immunosorbent assay; ametryn, deisopropylprometryn, demethylnorflurazon, flufenacet, norflurazon, pendimethalin, propachlor, propanil, propazine, prometryn, terbutryn, and 3-trifluoromethylaniline were not detected]

\begin{tabular}{|c|c|c|c|c|c|c|c|c|c|c|c|c|c|c|c|c|c|c|c|c|}
\hline \multirow[b]{2}{*}{$\begin{array}{l}\text { Date of } \\
\text { collection } \\
\text { (month/ } \\
\text { day/year) }\end{array}$} & \multirow[b]{2}{*}{$\begin{array}{l}\text { Collec- } \\
\text { tion } \\
\text { time } \\
(24- \\
\text { hour) }\end{array}$} & \multirow[b]{2}{*}{$\begin{array}{l}\text { Sam- } \\
\text { ple } \\
\text { type }\end{array}$} & \multicolumn{18}{|c|}{ Concentration, in micrograms per liter } \\
\hline & & & $\begin{array}{l}\text { Aceto- } \\
\text { chlor }\end{array}$ & $\begin{array}{l}\text { Ala- } \\
\text { chlor }\end{array}$ & $\begin{array}{l}\text { Ala- } \\
\text { chlor } \\
\text { ESA } \\
\text { ELISA }\end{array}$ & $\begin{array}{l}\text { Atra- } \\
\text { zine }\end{array}$ & $\begin{array}{l}\text { De- } \\
\text { ethyl- } \\
\text { atra- } \\
\text { zine }\end{array}$ & $\begin{array}{l}\text { Deiso- } \\
\text { propyl- } \\
\text { atrazine }\end{array}$ & $\begin{array}{l}\text { Cyan- } \\
\text { azine }\end{array}$ & $\begin{array}{l}\text { Cyan- } \\
\text { azine } \\
\text { amide }\end{array}$ & $\begin{array}{c}\text { De- } \\
\text { methyl- } \\
\text { fluo- } \\
\text { metu- } \\
\text { ron }\end{array}$ & $\begin{array}{l}\text { Di- } \\
\text { meth- } \\
\text { enamid }\end{array}$ & $\begin{array}{l}\text { Fluo- } \\
\text { met- } \\
\text { uron }\end{array}$ & $\begin{array}{l}\text { Meto- } \\
\text { lachlor }\end{array}$ & $\begin{array}{l}\text { Metri- } \\
\text { buzin }\end{array}$ & $\begin{array}{c}\text { Moli- } \\
\text { nate }\end{array}$ & $\begin{array}{l}\text { Pro- } \\
\text { meton }\end{array}$ & $\begin{array}{c}\text { Sima- } \\
\text { zine }\end{array}$ & $\begin{array}{c}\text { Tri- } \\
\text { fluralin }\end{array}$ & $\begin{array}{l}\text { 3-triflu- } \\
\text { oro- } \\
\text { methyl- } \\
\text { phenyl- } \\
\text { urea }\end{array}$ \\
\hline $07 / 25 / 02$ & 1400 & $\mathrm{R}$ & 0.08 & $<0.05$ & -- & 0.90 & 0.17 & 0.06 & $<0.05$ & $<0.05$ & $<0.05$ & $<0.05$ & $<0.05$ & 0.16 & $<0.05$ & $<0.05$ & -- & 0.05 & $<0.05$ & $<0.05$ \\
\hline 08/21/02 & 1100 & $\mathrm{R}$ & $<.05$ & $<.05$ & -- & .40 & .12 & $<.05$ & $<.05$ & $<.05$ & $<.05$ & $<.05$ & $<.05$ & $<.05$ & $<.05$ & $<.05$ & -- & $<.05$ & $<.05$ & $<.05$ \\
\hline 09/17/02 & 1400 & $\mathrm{R}$ & $<.05$ & $<.05$ & -- & .22 & .07 & $<.05$ & $<.05$ & $<.05$ & $<.05$ & $<.05$ & $<.05$ & $<.05$ & $<.05$ & $<.05$ & -- & $<.05$ & $<.05$ & $<.05$ \\
\hline $10 / 16 / 02$ & 1500 & $\mathrm{R}$ & $<.05$ & $<.05$ & -- & .11 & $<.05$ & $<.05$ & $<.05$ & $<.05$ & $<.05$ & $<.05$ & $<.05$ & $<.05$ & $<.05$ & $<.05$ & -- & $<.05$ & $<.05$ & $<.05$ \\
\hline $11 / 19 / 02$ & 1400 & $\mathrm{R}$ & $<.05$ & $<.05$ & -- & .07 & $<.05$ & $<.05$ & $<.05$ & $<.05$ & $<.05$ & $<.05$ & $<.05$ & $<.05$ & $<.05$ & $<.05$ & -- & .06 & $<.05$ & $<.05$ \\
\hline $11 / 19 / 02$ & 1400 & $\mathrm{~L}$ & $<.05$ & $<.05$ & -- & .06 & $<.05$ & $<.05$ & $<.05$ & $<.05$ & $<.05$ & $<.05$ & $<.05$ & $<.05$ & $<.05$ & $<.05$ & -- & .06 & $<.05$ & $<.05$ \\
\hline $12 / 18 / 02$ & 1330 & $\mathrm{R}$ & $<.05$ & $<.05$ & -- & .06 & $<.05$ & $<.05$ & $<.05$ & $<.05$ & $<.05$ & $<.05$ & $<.05$ & $<.05$ & $<.05$ & $<.05$ & -- & .05 & $<.05$ & $<.05$ \\
\hline $12 / 18 / 02$ & 1330 & $\mathrm{~L}$ & $<.05$ & $<.05$ & -- & .05 & $<.05$ & $<.05$ & $<.05$ & $<.05$ & $<.05$ & $<.05$ & $<.05$ & $<.05$ & $<.05$ & $<.05$ & -- & .05 & $<.05$ & $<.05$ \\
\hline $01 / 15 / 03$ & 1400 & $\mathrm{R}$ & $<.05$ & $<.05$ & -- & .06 & $<.05$ & $<.05$ & $<.05$ & $<.05$ & $<.05$ & $<.05$ & $<.05$ & $<.05$ & $<.05$ & $<.05$ & -- & .30 & $<.05$ & $<.05$ \\
\hline 01/15/03 & 1400 & $\mathrm{~L}$ & $<.05$ & $<.05$ & -- & .06 & $<.05$ & $<.05$ & $<.05$ & $<.05$ & $<.05$ & $<.05$ & $<.05$ & $<.05$ & $<.05$ & $<.05$ & -- & .32 & $<.05$ & $<.05$ \\
\hline $02 / 20 / 03$ & 1330 & $\mathrm{R}$ & $<.05$ & .07 & -- & .05 & $<.05$ & $<.05$ & $<.05$ & $<.05$ & $<.05$ & $<.05$ & $<.05$ & $<.05$ & $<.05$ & $<.05$ & -- & $<.05$ & $<.05$ & $<.05$ \\
\hline 03/13/03 & 1200 & $\mathrm{R}$ & $<.05$ & .05 & -- & $<.05$ & $<.05$ & $<.05$ & $<.05$ & $<.05$ & $<.05$ & $<.05$ & $<.05$ & $<.05$ & $<.05$ & $<.05$ & -- & .06 & $<.05$ & $<.05$ \\
\hline 04/03/03 & 1100 & $\mathrm{R}$ & $<.05$ & $<.05$ & -- & .15 & $<.05$ & $<.05$ & $<.05$ & $<.05$ & $<.05$ & $<.05$ & $<.05$ & $<.05$ & $<.05$ & $<.05$ & -- & .10 & $<.05$ & $<.05$ \\
\hline $05 / 19 / 03$ & 1330 & $\mathrm{R}$ & .13 & $<.05$ & -- & 2.1 & .27 & .18 & $<.05$ & $<.05$ & $<.05$ & $<.05$ & $<.05$ & .39 & $<.05$ & $<.05$ & -- & .30 & $<.05$ & $<.05$ \\
\hline
\end{tabular}


Table 5. Results of analysis of selected acetamide herbicides and degradation products by liquid chromatography/mass spectrometry methods HPAA (1998-99), LCAA (1999-02), and LCPD (2002-03) at the U.S. Geological Survey Organic Geochemistry Research Laboratory, Lawrence, Kansas, for water samples collected from the Mississippi River at Baton Rouge, Louisiana, 1998-2003.

[R, regular sample; L, laboratory duplicate sample; --, no data; <, less than]

\begin{tabular}{|c|c|c|c|c|c|c|c|c|c|c|c|c|c|c|c|}
\hline \multirow[b]{2}{*}{$\begin{array}{l}\text { Date of } \\
\text { collection } \\
\text { (month/ } \\
\text { day/year) }\end{array}$} & \multirow[b]{2}{*}{$\begin{array}{l}\text { Collec- } \\
\text { tion time } \\
\text { (24-hour) }\end{array}$} & \multirow[b]{2}{*}{$\begin{array}{c}\text { Sample } \\
\text { type }\end{array}$} & \multicolumn{13}{|c|}{ Concentrations, in micrograms per liter } \\
\hline & & & $\begin{array}{l}\text { Aceto- } \\
\text { chlor }\end{array}$ & $\begin{array}{l}\text { Aceto- } \\
\text { chlor } \\
\text { ethane- } \\
\text { sulfonic } \\
\text { acid }\end{array}$ & $\begin{array}{l}\text { Aceto- } \\
\text { chlor } \\
\text { oxanilic } \\
\text { acid }\end{array}$ & $\begin{array}{l}\text { Aceto- } \\
\text { chlor } \\
\text { sulfynil } \\
\text { acetic } \\
\text { acid }\end{array}$ & Alachlor & $\begin{array}{c}\text { Alachlor } \\
\text { ethane- } \\
\text { sulfonic } \\
\text { acid }\end{array}$ & $\begin{array}{c}\text { Alachlor } \\
\text { oxanilic } \\
\text { acid }\end{array}$ & $\begin{array}{c}\text { Alachlor } \\
\text { sulfynil } \\
\text { acetic } \\
\text { acid }\end{array}$ & $\begin{array}{l}\text { Alachlor } \\
\text { ethane- } \\
\text { sulfonic } \\
\text { acid 2nd } \\
\text { amide }\end{array}$ & $\begin{array}{l}\text { Dimeth- } \\
\text { enamid }\end{array}$ & $\begin{array}{l}\text { Dimeth- } \\
\text { enamid } \\
\text { ethane- } \\
\text { sulfonic } \\
\text { acid }\end{array}$ & $\begin{array}{l}\text { Dimeth- } \\
\text { enamid } \\
\text { oxanilic } \\
\text { acid }\end{array}$ & $\begin{array}{c}\text { Flufen- } \\
\text { acet }\end{array}$ \\
\hline $03 / 10 / 98$ & 1000 & $\mathrm{R}$ & -- & $<0.20$ & $<0.20$ & -- & -- & $<0.20$ & $<0.20$ & -- & -- & -- & -- & -- & -- \\
\hline 03/20/98 & 1010 & $\mathrm{R}$ & -- & $<.20$ & $<.20$ & -- & -- & $<.20$ & $<.20$ & -- & -- & -- & -- & -- & -- \\
\hline $04 / 10 / 98$ & 1400 & $\mathrm{R}$ & -- & $<.20$ & $<.20$ & -- & -- & .21 & $<.20$ & -- & -- & -- & -- & -- & -- \\
\hline $04 / 17 / 98$ & 1200 & $\mathrm{R}$ & -- & $<.20$ & $<.20$ & -- & -- & .22 & $<.20$ & -- & -- & -- & -- & -- & -- \\
\hline $04 / 22 / 98$ & 1040 & $\mathrm{R}$ & -- & $<.20$ & $<.20$ & -- & -- & .33 & $<.20$ & -- & -- & -- & -- & -- & -- \\
\hline 05/08/98 & 1200 & $\mathrm{R}$ & -- & $<.20$ & $<.20$ & -- & -- & $<.20$ & $<.20$ & -- & -- & -- & -- & -- & -- \\
\hline $07 / 24 / 98$ & 1100 & $\mathrm{R}$ & -- & .53 & .49 & -- & -- & .38 & $<.20$ & -- & -- & -- & -- & -- & -- \\
\hline 08/07/98 & 1105 & $\mathrm{R}$ & -- & .33 & .32 & -- & -- & .38 & $<.20$ & -- & -- & -- & -- & -- & -- \\
\hline 08/13/98 & 1345 & $\mathrm{R}$ & -- & $<.20$ & .21 & -- & -- & .22 & $<.20$ & -- & -- & -- & -- & -- & -- \\
\hline 08/13/98 & 1345 & $\mathrm{~L}$ & -- & $<.20$ & $<.20$ & -- & -- & .23 & $<.20$ & -- & -- & -- & -- & -- & -- \\
\hline $08 / 27 / 98$ & 1120 & $\mathrm{R}$ & -- & .40 & $<.20$ & -- & -- & .44 & $<.20$ & -- & -- & -- & -- & -- & -- \\
\hline $09 / 11 / 98$ & 1535 & $\mathrm{R}$ & -- & $<.20$ & $<.20$ & -- & -- & $<.20$ & $<.20$ & -- & -- & -- & -- & -- & -- \\
\hline 09/18/98 & 1045 & $\mathrm{R}$ & -- & .31 & $<.20$ & -- & -- & .27 & $<.20$ & -- & -- & -- & -- & -- & -- \\
\hline $10 / 02 / 98$ & 1515 & $\mathrm{R}$ & -- & $<.20$ & $<.20$ & -- & -- & .23 & $<.20$ & -- & -- & -- & -- & -- & -- \\
\hline $10 / 23 / 98$ & 1120 & $\mathrm{R}$ & -- & $<.20$ & $<.20$ & -- & -- & $<.20$ & $<.20$ & -- & -- & -- & -- & -- & -- \\
\hline $10 / 30 / 98$ & 1330 & $\mathrm{R}$ & -- & $<.20$ & $<.20$ & -- & -- & $<.20$ & $<.20$ & -- & -- & -- & -- & -- & -- \\
\hline $11 / 10 / 98$ & 1430 & $\mathrm{R}$ & -- & $<.20$ & $<.20$ & -- & -- & $<.20$ & $<.20$ & -- & -- & -- & -- & -- & -- \\
\hline $11 / 23 / 98$ & 0900 & $\mathrm{R}$ & -- & $<.20$ & $<.20$ & -- & -- & $<.20$ & $<.20$ & -- & -- & -- & -- & -- & -- \\
\hline $12 / 23 / 98$ & 0745 & $\mathrm{R}$ & -- & $<.20$ & $<.20$ & -- & -- & .32 & $<.20$ & -- & -- & -- & -- & -- & -- \\
\hline
\end{tabular}


Table 5. Results of analysis of selected acetamide herbicides and degradation products by liquid chromatography/mass spectrometry methods HPAA (1998-99) LCAA (1999-02), and LCPD (2002-03) at the U.S. Geological Survey Organic Geochemistry Research Laboratory, Lawrence, Kansas, for water samples collected from the Mississippi River at Baton Rouge, Louisiana, 1998-2003.-Continued

[R, regular sample; L, laboratory duplicate sample; --, no data; <, less than]

\begin{tabular}{|c|c|c|c|c|c|c|c|c|c|c|c|c|c|c|c|}
\hline \multirow[b]{2}{*}{$\begin{array}{l}\text { Date of } \\
\text { collection } \\
\text { (month/ } \\
\text { day/year) }\end{array}$} & \multirow[b]{2}{*}{$\begin{array}{l}\text { Collec- } \\
\text { tion time } \\
\text { (24-hour) }\end{array}$} & \multirow[b]{2}{*}{$\begin{array}{l}\text { Sample } \\
\text { type }\end{array}$} & \multicolumn{13}{|c|}{ Concentrations, in micrograms per liter } \\
\hline & & & $\begin{array}{l}\text { Aceto- } \\
\text { chlor }\end{array}$ & $\begin{array}{l}\text { Aceto- } \\
\text { chlor } \\
\text { ethane- } \\
\text { sulfonic } \\
\text { acid }\end{array}$ & $\begin{array}{l}\text { Aceto- } \\
\text { chlor } \\
\text { oxanilic } \\
\text { acid }\end{array}$ & $\begin{array}{l}\text { Aceto- } \\
\text { chlor } \\
\text { sulfynil } \\
\text { acetic } \\
\text { acid }\end{array}$ & Alachlor & $\begin{array}{l}\text { Alachlor } \\
\text { ethane- } \\
\text { sulfonic } \\
\text { acid }\end{array}$ & $\begin{array}{c}\text { Alachlor } \\
\text { oxanilic } \\
\text { acid }\end{array}$ & $\begin{array}{l}\text { Alachlor } \\
\text { sulfynil } \\
\text { acetic } \\
\text { acid }\end{array}$ & $\begin{array}{l}\text { Alachlor } \\
\text { ethane- } \\
\text { sulfonic } \\
\text { acid 2nd } \\
\text { amide }\end{array}$ & $\begin{array}{l}\text { Dimeth- } \\
\text { enamid }\end{array}$ & $\begin{array}{l}\text { Dimeth- } \\
\text { enamid } \\
\text { ethane- } \\
\text { sulfonic } \\
\text { acid }\end{array}$ & $\begin{array}{l}\text { Dimeth- } \\
\text { enamid } \\
\text { oxanilic } \\
\text { acid }\end{array}$ & $\begin{array}{c}\text { Flufen- } \\
\text { acet }\end{array}$ \\
\hline $01 / 05 / 99$ & 1100 & $\mathrm{R}$ & -- & $<0.20$ & $<0.20$ & -- & -- & 0.22 & $<0.20$ & -- & -- & -- & -- & -- & -- \\
\hline 01/20/99 & 1030 & $\mathrm{R}$ & -- & $<.20$ & $<.20$ & -- & -- & $<.20$ & $<.20$ & -- & -- & -- & -- & -- & -- \\
\hline $01 / 20 / 99$ & 1030 & $\mathrm{~L}$ & -- & $<.20$ & $<.20$ & -- & -- & $<.20$ & $<.20$ & -- & -- & -- & -- & -- & -- \\
\hline 02/03/99 & 1000 & $\mathrm{R}$ & -- & $<.20$ & $<.20$ & -- & -- & .20 & $<.20$ & -- & -- & -- & -- & -- & -- \\
\hline $02 / 23 / 99$ & 1000 & $\mathrm{R}$ & -- & $<.20$ & $<.20$ & -- & -- & $<.20$ & $<.20$ & -- & -- & -- & -- & -- & -- \\
\hline 02/23/99 & 1000 & $\mathrm{~L}$ & -- & $<.20$ & $<.20$ & -- & -- & $<.20$ & $<.20$ & -- & -- & -- & -- & -- & -- \\
\hline 03/12/99 & 0945 & $\mathrm{R}$ & -- & $<.20$ & $<.20$ & -- & -- & $<.20$ & $<.20$ & -- & -- & -- & -- & -- & -- \\
\hline 03/26/99 & 1000 & $\mathrm{R}$ & -- & $<.20$ & $<.20$ & -- & -- & $<.20$ & $<.20$ & -- & -- & -- & -- & -- & -- \\
\hline $04 / 02 / 99$ & 0800 & $\mathrm{R}$ & -- & $<.20$ & $<.20$ & -- & -- & $<.20$ & $<.20$ & -- & -- & -- & -- & -- & -- \\
\hline 04/15/99 & 1515 & $\mathrm{R}$ & -- & $<.20$ & $<.20$ & -- & -- & $<.20$ & $<.20$ & -- & -- & -- & -- & -- & -- \\
\hline 04/23/99 & 1245 & $\mathrm{R}$ & -- & $<.20$ & $<.20$ & -- & -- & $<.20$ & $<.20$ & -- & -- & -- & -- & -- & -- \\
\hline 04/30/99 & 1500 & $\mathrm{R}$ & -- & .10 & .11 & -- & -- & .13 & .05 & -- & -- & -- & $<0.05$ & $<0.05$ & -- \\
\hline 05/07/99 & 1000 & $\mathrm{R}$ & -- & .11 & .12 & -- & -- & .11 & $<.05$ & -- & -- & -- & $<.05$ & $<.05$ & -- \\
\hline 05/17/99 & 1000 & $\mathrm{R}$ & -- & .17 & .20 & -- & -- & .10 & $<.05$ & -- & -- & -- & $<.05$ & $<.05$ & -- \\
\hline 05/25/99 & 1100 & $\mathrm{R}$ & -- & .13 & .20 & -- & -- & .10 & .05 & -- & -- & -- & $<.05$ & $<.05$ & -- \\
\hline 06/03/99 & 1030 & $\mathrm{R}$ & -- & .29 & .41 & -- & -- & .13 & .07 & -- & -- & -- & $<.05$ & .05 & -- \\
\hline 06/10/99 & 1200 & $\mathrm{R}$ & -- & .42 & .60 & -- & -- & .26 & $<.20$ & -- & -- & -- & -- & -- & -- \\
\hline 06/18/99 & 1100 & $\mathrm{R}$ & -- & .33 & .42 & -- & -- & .23 & $<.20$ & -- & -- & -- & -- & -- & -- \\
\hline 06/25/99 & 1145 & $\mathrm{R}$ & -- & .45 & .56 & -- & -- & .21 & .12 & -- & -- & -- & .07 & .11 & -- \\
\hline 07/09/99 & 1530 & $\mathrm{R}$ & -- & $<.20$ & .32 & -- & -- & $<.20$ & $<.20$ & -- & -- & -- & -- & -- & -- \\
\hline
\end{tabular}


Table 5. Results of analysis of selected acetamide herbicides and degradation products by liquid chromatography/mass spectrometry methods HPAA (1998-99), LCAA (1999-02), and LCPD (2002-03) at the U.S. Geological Survey Organic Geochemistry Research Laboratory, Lawrence, Kansas, for water samples collected from the Mississippi River at Baton Rouge, Louisiana, 1998-2003.-Continued

[R, regular sample; L, laboratory duplicate sample; --, no data; <, less than]

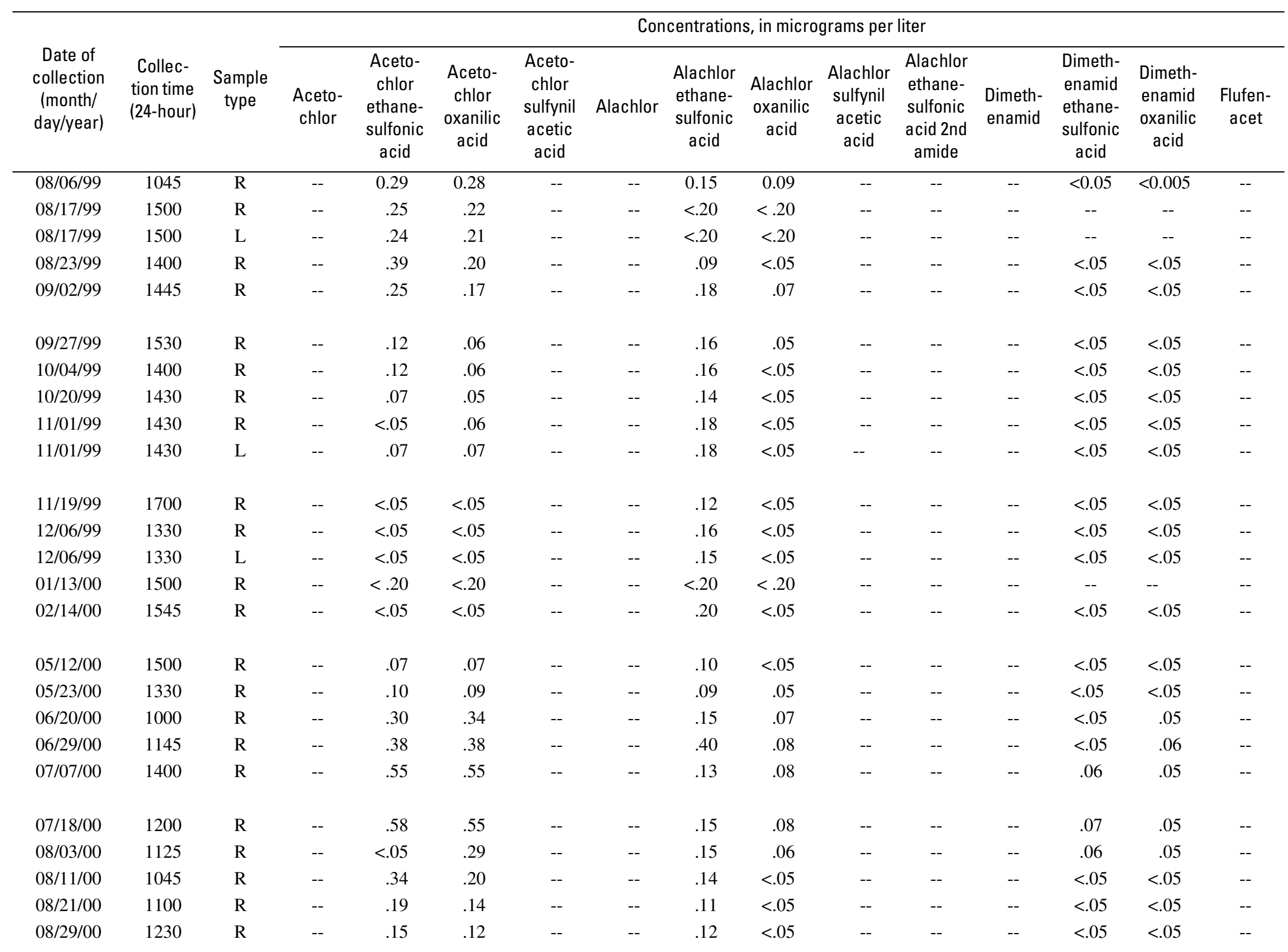


Table 5. Results of analysis of selected acetamide herbicides and degradation products by liquid chromatography/mass spectrometry methods HPAA (1998-99), LCAA (1999-02), and LCPD (2002-03) at the U.S. Geological Survey Organic Geochemistry Research Laboratory, Lawrence, Kansas, for water samples collected from the Mississippi River at Baton Rouge, Louisiana, 1998-2003.-Continued

[R, regular sample; L, laboratory duplicate sample; --, no data; <, less than]

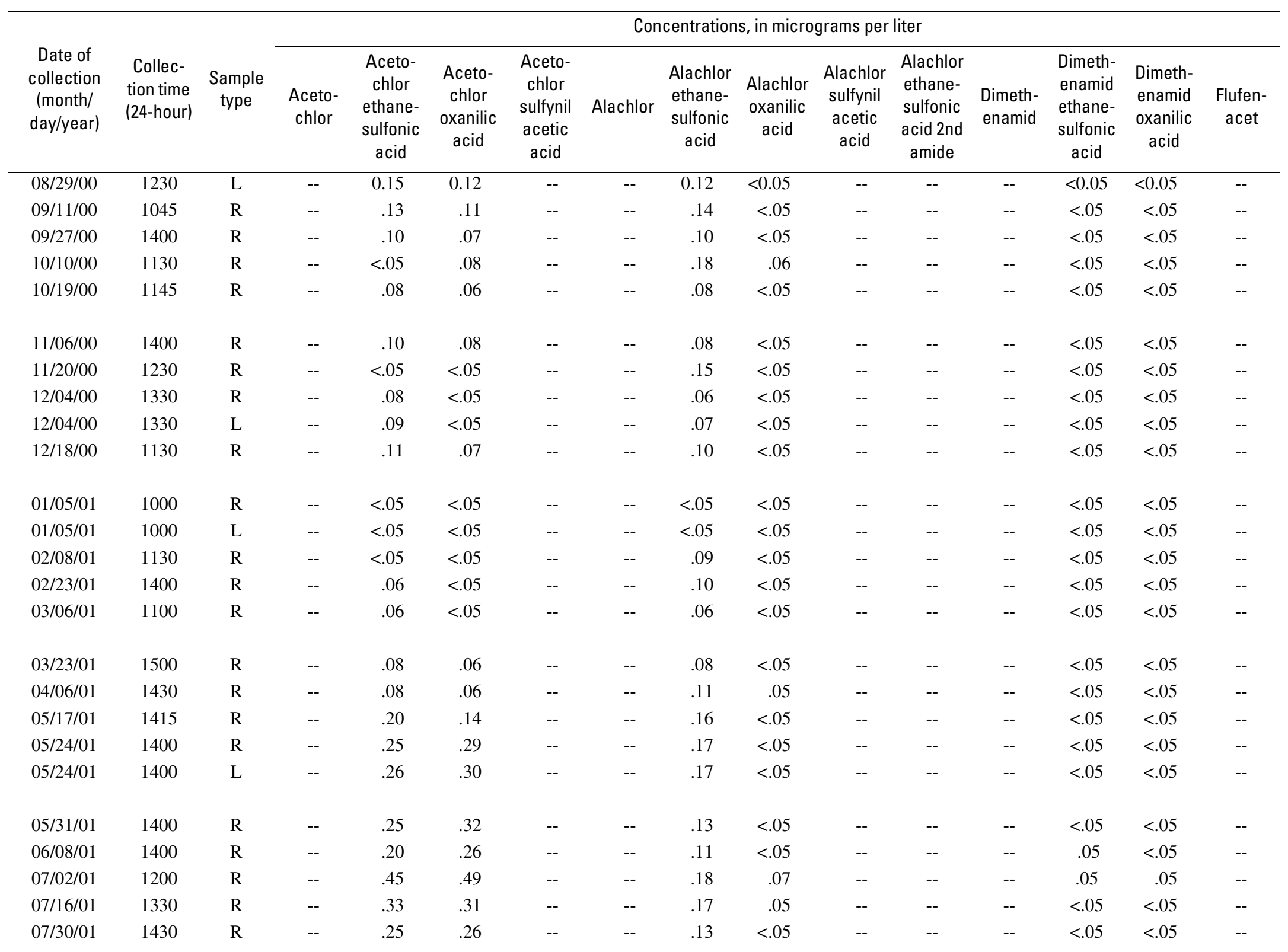


Table 5. Results of analysis of selected acetamide herbicides and degradation products by liquid chromatography/mass spectrometry methods HPAA (1998-99), LCAA (1999-02), and LCPD (2002-03) at the U.S. Geological Survey Organic Geochemistry Research Laboratory, Lawrence, Kansas, for water samples collected from the Mississippi River at Baton Rouge, Louisiana, 1998-2003.-Continued

[R, regular sample; L, laboratory duplicate sample; --, no data; <, less than]

\begin{tabular}{|c|c|c|c|c|c|c|c|c|c|c|c|c|c|c|c|}
\hline \multirow[b]{2}{*}{$\begin{array}{l}\text { Date of } \\
\text { collection } \\
\text { (month/ } \\
\text { day/year) }\end{array}$} & \multirow[b]{2}{*}{$\begin{array}{l}\text { Collec- } \\
\text { tion time } \\
\text { (24-hour) }\end{array}$} & \multirow[b]{2}{*}{$\begin{array}{c}\text { Sample } \\
\text { type }\end{array}$} & \multicolumn{13}{|c|}{ Concentrations, in micrograms per liter } \\
\hline & & & $\begin{array}{l}\text { Aceto- } \\
\text { chlor }\end{array}$ & $\begin{array}{l}\text { Aceto- } \\
\text { chlor } \\
\text { ethane- } \\
\text { sulfonic } \\
\text { acid }\end{array}$ & $\begin{array}{l}\text { Aceto- } \\
\text { chlor } \\
\text { oxanilic } \\
\text { acid }\end{array}$ & $\begin{array}{l}\text { Aceto- } \\
\text { chlor } \\
\text { sulfynil } \\
\text { acetic } \\
\text { acid }\end{array}$ & Alachlor & $\begin{array}{l}\text { Alachlor } \\
\text { ethane- } \\
\text { sulfonic } \\
\text { acid }\end{array}$ & $\begin{array}{c}\text { Alachlor } \\
\text { oxanilic } \\
\text { acid }\end{array}$ & $\begin{array}{l}\text { Alachlor } \\
\text { sulfynil } \\
\text { acetic } \\
\text { acid }\end{array}$ & $\begin{array}{l}\text { Alachlor } \\
\text { ethane- } \\
\text { sulfonic } \\
\text { acid 2nd } \\
\text { amide }\end{array}$ & $\begin{array}{l}\text { Dimeth- } \\
\text { enamid }\end{array}$ & $\begin{array}{l}\text { Dimeth- } \\
\text { enamid } \\
\text { ethane- } \\
\text { sulfonic } \\
\text { acid }\end{array}$ & $\begin{array}{l}\text { Dimeth- } \\
\text { enamid } \\
\text { oxanilic } \\
\text { acid }\end{array}$ & $\begin{array}{c}\text { Flufen- } \\
\text { acet }\end{array}$ \\
\hline $07 / 30 / 01$ & 1430 & $\mathrm{~L}$ & -- & 0.28 & 0.28 & -- & -- & 0.13 & $<0.05$ & -- & -- & -- & $<0.05$ & $<0.05$ & -- \\
\hline 08/13/01 & 1130 & $\mathrm{R}$ & -- & .20 & .20 & -- & -- & .14 & $<.05$ & -- & -- & -- & $<.05$ & $<.05$ & -- \\
\hline $08 / 28 / 01$ & 1330 & $\mathrm{R}$ & -- & .08 & .11 & -- & -- & .07 & $<.05$ & -- & -- & -- & $<.05$ & $<.05$ & -- \\
\hline $09 / 18 / 01$ & 1100 & $\mathrm{R}$ & -- & .07 & .08 & -- & -- & .07 & .05 & -- & -- & -- & $<.05$ & $<.05$ & -- \\
\hline $10 / 01 / 01$ & 1330 & $\mathrm{R}$ & -- & $<.05$ & .06 & -- & -- & .11 & $<.05$ & -- & -- & -- & $<.05$ & $<.05$ & -- \\
\hline $10 / 16 / 01$ & 1200 & $\mathrm{R}$ & -- & .05 & .07 & -- & -- & .11 & $<.05$ & -- & -- & -- & $<.05$ & $<.05$ & -- \\
\hline $10 / 16 / 01$ & 1200 & $\mathrm{~L}$ & -- & .05 & .07 & -- & -- & .11 & $<.05$ & -- & -- & -- & $<.05$ & $<.05$ & -- \\
\hline $10 / 31 / 01$ & 1300 & $\mathrm{R}$ & -- & .05 & .07 & -- & -- & .09 & $<.05$ & -- & -- & -- & $<.05$ & $<.05$ & -- \\
\hline $11 / 14 / 01$ & 1300 & $\mathrm{R}$ & -- & .16 & .10 & -- & -- & .13 & $<.05$ & -- & -- & -- & $<.05$ & $<.05$ & -- \\
\hline $11 / 14 / 01$ & 1300 & $\mathrm{~L}$ & -- & .13 & .10 & -- & -- & .17 & $<.05$ & -- & -- & -- & $<.05$ & $<.05$ & -- \\
\hline $11 / 28 / 01$ & 1200 & $\mathrm{R}$ & -- & .19 & .10 & -- & -- & .15 & $<.05$ & -- & -- & -- & $<.05$ & $<.05$ & -- \\
\hline $12 / 10 / 01$ & 1300 & $\mathrm{R}$ & -- & .05 & .05 & $<0.05$ & -- & $<.05$ & $<.05$ & $<0.05$ & -- & -- & $<.05$ & $<.05$ & -- \\
\hline 01/03/02 & 1140 & $\mathrm{R}$ & -- & $<.05$ & $<.05$ & $<.05$ & -- & .05 & $<.05$ & $<.05$ & -- & -- & $<.05$ & $<.05$ & -- \\
\hline 01/03/02 & 1140 & $\mathrm{~L}$ & -- & $<.05$ & $<.05$ & $<.05$ & -- & .06 & $<.05$ & $<.05$ & -- & -- & $<.05$ & $<.05$ & -- \\
\hline $01 / 17 / 02$ & 1100 & $\mathrm{R}$ & -- & .09 & $<.05$ & $<.05$ & -- & .09 & $<.05$ & $<.05$ & -- & -- & $<.05$ & $<.05$ & -- \\
\hline $02 / 04 / 02$ & 1300 & $\mathrm{R}$ & -- & .06 & $<.05$ & $<.05$ & -- & .06 & $<.05$ & $<.05$ & -- & -- & $<.05$ & $<.05$ & -- \\
\hline $02 / 28 / 02$ & 1230 & $\mathrm{R}$ & -- & .06 & $<.05$ & $<.05$ & -- & .09 & $<.05$ & $<.05$ & -- & -- & $<.05$ & $<.05$ & -- \\
\hline $02 / 28 / 02$ & 1230 & $\mathrm{~L}$ & -- & .08 & $<.05$ & $<.05$ & -- & .07 & $<.05$ & $<.05$ & -- & -- & $<.05$ & $<.05$ & -- \\
\hline $03 / 11 / 02$ & 1300 & $\mathrm{R}$ & -- & .07 & $<.05$ & $<.05$ & -- & .12 & $<.05$ & $<.05$ & -- & -- & $<.05$ & $<.05$ & -- \\
\hline 03/28/02 & 1300 & $\mathrm{R}$ & -- & .06 & $<.05$ & $<.05$ & -- & .08 & $<.05$ & $<.05$ & -- & -- & $<.05$ & $<.05$ & -- \\
\hline $03 / 28 / 02$ & 1300 & $\mathrm{~L}$ & -- & .05 & $<.05$ & $<.05$ & -- & .07 & $<.05$ & $<.05$ & -- & -- & $<.05$ & $<.05$ & -- \\
\hline $04 / 12 / 02$ & 1115 & $\mathrm{R}$ & -- & $<.05$ & $<.05$ & $<.05$ & -- & .06 & $<.05$ & $<.05$ & -- & -- & $<.05$ & $<.05$ & -- \\
\hline $04 / 12 / 02$ & 1115 & $\mathrm{~L}$ & -- & $<.05$ & $<.05$ & $<.05$ & -- & .05 & $<.05$ & $<.05$ & -- & -- & $<.05$ & $<.05$ & -- \\
\hline $05 / 02 / 02$ & 1300 & $\mathrm{R}$ & -- & .06 & .05 & $<.05$ & -- & .10 & $<.05$ & $<.05$ & -- & -- & $<.05$ & $<.05$ & -- \\
\hline 05/15/02 & 1300 & $\mathrm{R}$ & -- & .11 & .12 & $<.05$ & -- & .07 & $<.05$ & $<.05$ & -- & -- & $<.05$ & $<.05$ & -- \\
\hline
\end{tabular}


Table 5. Results of analysis of selected acetamide herbicides and degradation products by liquid chromatography/mass spectrometry methods HPAA (1998-99), LCAA (1999-02), and LCPD (2002-03) at the U.S. Geological Survey Organic Geochemistry Research Laboratory, Lawrence, Kansas, for water samples collected from the Mississippi River at Baton Rouge, Louisiana, 1998-2003.-Continued

[R, regular sample; L, laboratory duplicate sample; --, no data; <, less than]

\begin{tabular}{|c|c|c|c|c|c|c|c|c|c|c|c|c|c|c|c|}
\hline \multirow[b]{2}{*}{$\begin{array}{l}\text { Date of } \\
\text { collection } \\
\text { (month/ } \\
\text { day/year) }\end{array}$} & \multirow[b]{2}{*}{$\begin{array}{l}\text { Collec- } \\
\text { tion time } \\
\text { (24-hour) }\end{array}$} & \multirow[b]{2}{*}{$\begin{array}{l}\text { Sample } \\
\text { type }\end{array}$} & \multicolumn{13}{|c|}{ Concentrations, in micrograms per liter } \\
\hline & & & $\begin{array}{l}\text { Aceto- } \\
\text { chlor }\end{array}$ & $\begin{array}{l}\text { Aceto- } \\
\text { chlor } \\
\text { ethane- } \\
\text { sulfonic } \\
\text { acid }\end{array}$ & $\begin{array}{l}\text { Aceto- } \\
\text { chlor } \\
\text { oxanilic } \\
\text { acid }\end{array}$ & $\begin{array}{l}\text { Aceto- } \\
\text { chlor } \\
\text { sulfynil } \\
\text { acetic } \\
\text { acid }\end{array}$ & Alachlor & $\begin{array}{l}\text { Alachlor } \\
\text { ethane- } \\
\text { sulfonic } \\
\text { acid }\end{array}$ & $\begin{array}{c}\text { Alachlor } \\
\text { oxanilic } \\
\text { acid }\end{array}$ & $\begin{array}{l}\text { Alachlor } \\
\text { sulfynil } \\
\text { acetic } \\
\text { acid }\end{array}$ & $\begin{array}{l}\text { Alachlor } \\
\text { ethane- } \\
\text { sulfonic } \\
\text { acid 2nd } \\
\text { amide }\end{array}$ & $\begin{array}{l}\text { Dimeth- } \\
\text { enamid }\end{array}$ & $\begin{array}{l}\text { Dimeth- } \\
\text { enamid } \\
\text { ethane- } \\
\text { sulfonic } \\
\text { acid }\end{array}$ & $\begin{array}{l}\text { Dimeth- } \\
\text { enamid } \\
\text { oxanilic } \\
\text { acid }\end{array}$ & $\begin{array}{c}\text { Flufen- } \\
\text { acet }\end{array}$ \\
\hline $05 / 15 / 02$ & 1300 & $\mathrm{~L}$ & -- & 0.11 & 0.11 & $<0.05$ & -- & 0.07 & $<0.05$ & $<0.05$ & -- & -- & $<0.05$ & $<0.05$ & -- \\
\hline 06/05/02 & 1200 & $\mathrm{R}$ & -- & .20 & .22 & .17 & -- & .07 & $<.05$ & $<.05$ & -- & -- & $<.05$ & $<.05$ & -- \\
\hline 06/05/02 & 1200 & $\mathrm{~L}$ & -- & .20 & .22 & .17 & -- & .06 & $<.05$ & $<.05$ & -- & -- & $<.05$ & $<.05$ & -- \\
\hline $06 / 11 / 02$ & 1030 & $\mathrm{R}$ & -- & .17 & .15 & $<.05$ & -- & .06 & $<.05$ & $<.05$ & -- & -- & $<.05$ & $<.05$ & -- \\
\hline 07/02/02 & 1130 & $\mathrm{R}$ & -- & .32 & .38 & $<.05$ & -- & .09 & $<.05$ & $<.05$ & -- & -- & .06 & $<.05$ & -- \\
\hline 07/02/02 & 1130 & $\mathrm{~L}$ & -- & .35 & .43 & $<.05$ & -- & .08 & $<.05$ & $<.05$ & -- & -- & .06 & $<.05$ & -- \\
\hline $07 / 25 / 02$ & 1400 & $\mathrm{R}$ & -- & .13 & .12 & $<.05$ & -- & .09 & $<.05$ & $<.05$ & -- & -- & $<.05$ & $<.05$ & -- \\
\hline 08/21/02 & 1100 & $\mathrm{R}$ & -- & .10 & .10 & $<.05$ & -- & .09 & $<.05$ & $<.05$ & -- & -- & $<.05$ & $<.05$ & -- \\
\hline 09/17/02 & 1400 & $\mathrm{R}$ & -- & .11 & .11 & $<.05$ & -- & .12 & $<.05$ & $<.05$ & -- & -- & $<.05$ & $<.05$ & -- \\
\hline $11 / 19 / 02$ & 1400 & $\mathrm{R}$ & -- & $<.05$ & $<.05$ & $<.05$ & -- & .05 & $<.05$ & $<.05$ & -- & -- & $<.05$ & $<.05$ & -- \\
\hline $12 / 18 / 02$ & 1330 & $\mathrm{R}$ & $<0.02$ & $<.02$ & .03 & $<.02$ & $<0.02$ & .03 & .02 & $<.02$ & $<0.02$ & $<0.02$ & $<.02$ & $<.02$ & $<0.02$ \\
\hline 01/15/03 & 1400 & $\mathrm{R}$ & $<.02$ & .04 & .06 & $<.02$ & $<.02$ & .04 & .03 & $<.02$ & $<.02$ & $<.02$ & $<.02$ & $<.02$ & $<.02$ \\
\hline $02 / 20 / 03$ & 1330 & $\mathrm{R}$ & $<.02$ & $<.02$ & .04 & $<.02$ & $<.02$ & .08 & $<.02$ & $<.02$ & $<.02$ & $<.02$ & $<.02$ & $<.02$ & $<.02$ \\
\hline 03/13/03 & 1200 & $\mathrm{R}$ & $<.02$ & $<.02$ & $<.02$ & $<.02$ & $<.02$ & .03 & $<.02$ & $<.02$ & $<.02$ & $<.02$ & $<.02$ & $<.02$ & $<.02$ \\
\hline 04/03/03 & 1100 & $\mathrm{R}$ & $<.02$ & .06 & .03 & $<.02$ & $<.02$ & .08 & $<.02$ & $<.02$ & $<.02$ & $<.02$ & $<.02$ & $<.02$ & $<.02$ \\
\hline 05/19/03 & 1330 & $\mathrm{R}$ & .11 & .08 & .11 & .10 & $<.02$ & .09 & .02 & $<.02$ & $<.02$ & .06 & .02 & $<.02$ & $<.02$ \\
\hline 06/03/03 & 1230 & $\mathrm{R}$ & .14 & .14 & .21 & .09 & $<.02$ & .05 & .03 & $<.02$ & .03 & .04 & .06 & .02 & $<.02$ \\
\hline 06/19/03 & 0830 & $\mathrm{R}$ & .07 & .12 & .13 & .11 & $<.02$ & .07 & .02 & $<.02$ & $<.02$ & .02 & .02 & .02 & $<.02$ \\
\hline 07/03/03 & 1330 & $\mathrm{R}$ & .07 & .24 & .27 & .19 & $<.02$ & .05 & .03 & $<.02$ & $<.02$ & $<.02$ & .02 & $<.02$ & $<.02$ \\
\hline 07/15/03 & 1230 & $\mathrm{R}$ & $<.02$ & .13 & .14 & .10 & $<.02$ & .07 & .03 & $<.02$ & $<.02$ & $<.02$ & .03 & $<.02$ & $<.02$ \\
\hline 09/29/03 & 1300 & $\mathrm{R}$ & $<.02$ & .12 & .10 & .03 & $<.02$ & .05 & .02 & $<.02$ & $<.02$ & $<.02$ & .02 & $<.02$ & $<.02$ \\
\hline $10 / 15 / 03$ & 1430 & $\mathrm{R}$ & $<.02$ & .05 & .04 & .06 & $<.02$ & .03 & $<.02$ & $<.02$ & $<.02$ & $<.02$ & .11 & $<.02$ & $<.02$ \\
\hline
\end{tabular}


Table 5. Results of analysis of selected acetamide herbicides and degradation products by liquid chromatography/mass spectrometry methods HPAA (1998-99), LCAA (1999-02), and LCPD (2002-03) at the U.S. Geological Survey Organic Geochemistry Research Laboratory, Lawrence, Kansas, for water samples collected from the Mississippi River at Baton Rouge, Louisiana, 1998-2003.-Continued

[R, regular sample; L, laboratory duplicate sample; --, no data; <, less than]

\begin{tabular}{|c|c|c|c|c|c|c|c|c|c|c|c|c|c|c|c|}
\hline \multirow[b]{2}{*}{$\begin{array}{l}\text { Date of } \\
\text { collection } \\
\text { (month/ } \\
\text { day/year) }\end{array}$} & \multirow[b]{2}{*}{$\begin{array}{l}\text { Collec- } \\
\text { tion time } \\
\text { (24-hour) }\end{array}$} & \multirow[b]{2}{*}{$\begin{array}{l}\text { Sample } \\
\text { type }\end{array}$} & \multicolumn{13}{|c|}{ Concentrations, in micrograms per liter } \\
\hline & & & $\begin{array}{l}\text { Aceto- } \\
\text { chlor }\end{array}$ & $\begin{array}{l}\text { Aceto- } \\
\text { chlor } \\
\text { ethane- } \\
\text { sulfonic } \\
\text { acid }\end{array}$ & $\begin{array}{l}\text { Aceto- } \\
\text { chlor } \\
\text { oxanilic } \\
\text { acid }\end{array}$ & $\begin{array}{l}\text { Aceto- } \\
\text { chlor } \\
\text { sulfynil } \\
\text { acetic } \\
\text { acid }\end{array}$ & Alachlor & $\begin{array}{l}\text { Alachlor } \\
\text { ethane- } \\
\text { sulfonic } \\
\text { acid }\end{array}$ & $\begin{array}{c}\text { Alachlor } \\
\text { oxanilic } \\
\text { acid }\end{array}$ & $\begin{array}{l}\text { Alachlor } \\
\text { sulfynil } \\
\text { acetic } \\
\text { acid }\end{array}$ & $\begin{array}{l}\text { Alachlor } \\
\text { ethane- } \\
\text { sulfonic } \\
\text { acid 2nd } \\
\text { amide }\end{array}$ & $\begin{array}{l}\text { Dimeth- } \\
\text { enamid }\end{array}$ & $\begin{array}{l}\text { Dimeth- } \\
\text { enamid } \\
\text { ethane- } \\
\text { sulfonic } \\
\quad \text { acid }\end{array}$ & $\begin{array}{l}\text { Dimeth- } \\
\text { enamid } \\
\text { oxanilic } \\
\text { acid }\end{array}$ & $\begin{array}{c}\text { Flufen- } \\
\text { acet }\end{array}$ \\
\hline $11 / 05 / 03$ & 1230 & $\mathrm{R}$ & $<0.02$ & 0.05 & 0.05 & $<0.02$ & $<0.02$ & $<0.02$ & $<0.02$ & 0.02 & $<0.02$ & $<0.02$ & $<0.02$ & $<0.02$ & $<0.02$ \\
\hline $12 / 01 / 03$ & 1055 & $\mathrm{R}$ & $<.02$ & $<.02$ & $<.02$ & $<.02$ & $<.02$ & $<.02$ & $<.02$ & $<.02$ & $<.02$ & $<.02$ & $<.02$ & $<.02$ & $<.02$ \\
\hline $12 / 10 / 03$ & 1100 & $\mathrm{~L}$ & $<.02$ & .07 & .04 & $<.02$ & $<.02$ & $<.02$ & $<.02$ & $<.02$ & $<.02$ & $<.02$ & $<.02$ & $<.02$ & $<.02$ \\
\hline $12 / 30 / 03$ & 1030 & $\mathrm{R}$ & $<.02$ & .05 & .06 & .03 & $<.02$ & .04 & $<.02$ & $<.02$ & $<.02$ & $<.02$ & $<.02$ & $<.02$ & $<.02$ \\
\hline
\end{tabular}


Table 5. Results of analysis of selected acetamide herbicides and degradation products by liquid chromatography/mass spectrometry methods HPAA (1998-99), LCAA (1999-02), and LCPD (2002-03) at the U.S. Geological Survey Organic Geochemistry Research Laboratory, Lawrence, Kansas, for water samples collected from the Mississippi River at Baton Rouge, Louisiana, 1998-2003.—Continued

[R, regular sample; L, laboratory duplicate sample; --, no data; <, less than]

\begin{tabular}{|c|c|c|c|c|c|c|c|c|c|c|c|}
\hline \multirow[b]{2}{*}{$\begin{array}{l}\text { Date of } \\
\text { collection } \\
\text { (month/ } \\
\text { day/year) }\end{array}$} & \multirow[b]{2}{*}{$\begin{array}{l}\text { Collection } \\
\text { time } \\
\text { (24-hour) }\end{array}$} & \multirow[b]{2}{*}{ Sample type } & \multicolumn{9}{|c|}{ Concentrations, in micrograms per liter } \\
\hline & & & $\begin{array}{l}\text { Flufenacet } \\
\text { ethane- } \\
\text { sulfonic } \\
\text { acid }\end{array}$ & $\begin{array}{c}\text { Flufenacet } \\
\text { oxanilic } \\
\text { acid }\end{array}$ & $\begin{array}{l}\text { Meto- } \\
\text { lachlor }\end{array}$ & $\begin{array}{l}\text { Metolachlor } \\
\text { ethane- } \\
\text { sulfonic acid }\end{array}$ & $\begin{array}{l}\text { Metolachlor } \\
\text { oxanilic acid }\end{array}$ & $\begin{array}{l}\text { Metolachlor/ } \\
\text { acetochlor } \\
\text { ethane- } \\
\text { sulfonic acid } \\
\text { 2nd amide }\end{array}$ & Propachlor & $\begin{array}{l}\text { Propachlor } \\
\text { ethane- } \\
\text { sulfonic } \\
\text { acid }\end{array}$ & $\begin{array}{l}\text { Propachlor } \\
\text { oxanilic acid }\end{array}$ \\
\hline $03 / 10 / 98$ & 1000 & $\mathrm{R}$ & -- & -- & -- & 0.49 & $<0.20$ & -- & -- & -- & -- \\
\hline 03/20/98 & 1010 & $\mathrm{R}$ & -- & -- & -- & .49 & $<.20$ & -- & -- & -- & -- \\
\hline 03/27/98 & 1030 & $\mathrm{R}$ & -- & -- & -- & .39 & $<.20$ & -- & -- & -- & -- \\
\hline 04/10/98 & 1400 & $\mathrm{R}$ & -- & -- & -- & .66 & .22 & -- & -- & -- & -- \\
\hline $04 / 17 / 98$ & 1200 & $\mathrm{R}$ & -- & -- & -- & .57 & $<.20$ & -- & -- & -- & -- \\
\hline $04 / 22 / 98$ & 1040 & $\mathrm{R}$ & -- & -- & -- & .84 & .30 & -- & -- & -- & -- \\
\hline 05/08/98 & 1200 & $\mathrm{R}$ & -- & -- & -- & .84 & $<.20$ & -- & -- & -- & -- \\
\hline 07/24/98 & 1100 & $\mathrm{R}$ & -- & -- & -- & .78 & .57 & -- & -- & -- & -- \\
\hline 08/07/98 & 1105 & $\mathrm{R}$ & -- & -- & -- & .63 & .46 & -- & -- & -- & -- \\
\hline 08/07/98 & 1105 & $\mathrm{~L}$ & -- & -- & -- & .97 & .55 & -- & -- & -- & -- \\
\hline 08/13/98 & 1345 & $\mathrm{R}$ & -- & -- & -- & .45 & .33 & -- & -- & -- & -- \\
\hline 08/13/98 & 1345 & $\mathrm{~L}$ & -- & -- & -- & .69 & .34 & -- & -- & -- & -- \\
\hline 08/27/98 & 1120 & $\mathrm{R}$ & -- & -- & -- & 1.2 & .51 & -- & -- & -- & -- \\
\hline 09/11/98 & 1535 & $\mathrm{R}$ & -- & -- & -- & .67 & .23 & -- & -- & -- & -- \\
\hline 09/18/98 & 1045 & $\mathrm{R}$ & -- & -- & -- & .79 & .36 & -- & -- & -- & -- \\
\hline $10 / 02 / 98$ & 1515 & $\mathrm{R}$ & -- & -- & -- & .56 & $<.20$ & -- & -- & -- & -- \\
\hline $10 / 23 / 98$ & 1120 & $\mathrm{R}$ & -- & -- & -- & $<.20$ & $<.20$ & -- & -- & -- & -- \\
\hline $10 / 30 / 98$ & 1330 & $\mathrm{R}$ & -- & -- & -- & 1.3 & $<.20$ & -- & -- & -- & -- \\
\hline $11 / 10 / 98$ & 1430 & $\mathrm{R}$ & -- & -- & -- & .50 & $<.20$ & -- & -- & -- & -- \\
\hline $11 / 23 / 98$ & 0900 & $\mathrm{R}$ & -- & -- & -- & .44 & $<.20$ & -- & -- & -- & -- \\
\hline $12 / 23 / 98$ & 0745 & $\mathrm{R}$ & -- & -- & -- & .85 & .38 & -- & -- & -- & -- \\
\hline 01/05/99 & 1100 & $\mathrm{R}$ & -- & -- & -- & .85 & $<.20$ & -- & -- & -- & -- \\
\hline 01/20/99 & 1030 & $\mathrm{R}$ & -- & -- & -- & .28 & $<.20$ & -- & -- & -- & -- \\
\hline 01/20/99 & 1030 & $\mathrm{~L}$ & -- & -- & -- & .23 & $<.20$ & -- & -- & -- & -- \\
\hline 02/03/99 & 1000 & $\mathrm{R}$ & -- & -- & -- & .31 & $<.20$ & -- & -- & -- & -- \\
\hline
\end{tabular}


Table 5. Results of analysis of selected acetamide herbicides and degradation products by liquid chromatography/mass spectrometry methods HPAA (1998-99) LCAA (1999-02), and LCPD (2002-03) at the U.S. Geological Survey Organic Geochemistry Research Laboratory, Lawrence, Kansas, for water samples collected from the Mississippi River at Baton Rouge, Louisiana, 1998-2003.-Continued

[R, regular sample; L, laboratory duplicate sample; --, no data; <, less than]

\begin{tabular}{|c|c|c|c|c|c|c|c|c|c|c|c|}
\hline \multirow[b]{2}{*}{$\begin{array}{l}\text { Date of } \\
\text { collection } \\
\text { (month/ } \\
\text { day/year) }\end{array}$} & \multirow[b]{2}{*}{$\begin{array}{l}\text { Collection } \\
\text { time } \\
\text { (24-hour) }\end{array}$} & \multirow[b]{2}{*}{ Sample type } & \multicolumn{9}{|c|}{ Concentrations, in micrograms per liter } \\
\hline & & & $\begin{array}{l}\text { Flufenacet } \\
\text { ethane- } \\
\text { sulfonic } \\
\text { acid }\end{array}$ & $\begin{array}{c}\text { Flufenacet } \\
\text { oxanilic } \\
\text { acid }\end{array}$ & $\begin{array}{l}\text { Meto- } \\
\text { lachlor }\end{array}$ & $\begin{array}{l}\text { Metolachlor } \\
\text { ethane- } \\
\text { sulfonic acid }\end{array}$ & $\begin{array}{l}\text { Metolachlor } \\
\text { oxanilic acid }\end{array}$ & $\begin{array}{l}\text { Metolachlor/ } \\
\text { acetochlor } \\
\text { ethane- } \\
\text { sulfonic acid } \\
\text { 2nd amide }\end{array}$ & Propachlor & $\begin{array}{l}\text { Propachlor } \\
\text { ethane- } \\
\text { sulfonic } \\
\text { acid }\end{array}$ & $\begin{array}{l}\text { Propachlor } \\
\text { oxanilic acid }\end{array}$ \\
\hline $02 / 23 / 99$ & 1000 & $\mathrm{R}$ & -- & -- & -- & 0.43 & $<0.20$ & -- & -- & -- & -- \\
\hline $02 / 23 / 99$ & 1000 & $\mathrm{~L}$ & -- & -- & -- & .43 & $<.20$ & -- & -- & -- & -- \\
\hline 03/12/99 & 0945 & $\mathrm{R}$ & -- & -- & -- & .32 & $<.20$ & -- & -- & -- & -- \\
\hline 03/26/99 & 1000 & $\mathrm{R}$ & -- & -- & -- & .21 & $<.20$ & -- & -- & -- & -- \\
\hline $04 / 02 / 99$ & 0800 & $\mathrm{R}$ & -- & -- & -- & .22 & $<.20$ & -- & & & \\
\hline 04/08/99 & 1300 & $\mathrm{R}$ & -- & -- & -- & .22 & $<.20$ & -- & -- & -- & -- \\
\hline $04 / 15 / 99$ & 1515 & $\mathrm{R}$ & -- & -- & -- & .25 & $<.20$ & -- & -- & -- & -- \\
\hline $04 / 23 / 99$ & 1245 & $\mathrm{R}$ & -- & -- & -- & .26 & $<.20$ & -- & -- & -- & -- \\
\hline 04/30/99 & 1500 & $\mathrm{R}$ & -- & -- & -- & .46 & .18 & -- & -- & -- & -- \\
\hline 05/07/99 & 1000 & $\mathrm{R}$ & -- & -- & -- & .44 & .23 & -- & -- & -- & -- \\
\hline 05/17/99 & 1000 & $\mathrm{R}$ & -- & -- & -- & .51 & .27 & -- & -- & -- & -- \\
\hline 05/25/99 & 1100 & $\mathrm{R}$ & -- & -- & -- & .41 & .24 & -- & -- & -- & -- \\
\hline 06/03/99 & 1030 & $\mathrm{R}$ & -- & -- & -- & .53 & .30 & -- & -- & -- & -- \\
\hline 06/10/99 & 1200 & $\mathrm{R}$ & -- & -- & -- & .80 & .46 & -- & -- & -- & -- \\
\hline 06/18/99 & 1100 & $\mathrm{R}$ & -- & -- & -- & .78 & .30 & -- & -- & -- & -- \\
\hline $06 / 25 / 99$ & 1145 & $\mathrm{R}$ & -- & -- & -- & .80 & .46 & -- & -- & -- & -- \\
\hline 07/09/99 & 1530 & $\mathrm{R}$ & -- & -- & -- & .36 & .36 & -- & -- & -- & -- \\
\hline 07/16/99 & 1000 & $\mathrm{R}$ & -- & -- & -- & $<.20$ & $<.20$ & -- & -- & -- & -- \\
\hline 08/06/99 & 1045 & $\mathrm{R}$ & -- & -- & -- & .58 & .33 & -- & -- & -- & -- \\
\hline 08/17/99 & 1500 & $\mathrm{R}$ & -- & -- & -- & .48 & $<.20$ & -- & -- & -- & -- \\
\hline 08/17/99 & 1500 & $\mathrm{~L}$ & -- & -- & -- & .47 & $<.20$ & -- & -- & -- & -- \\
\hline $08 / 23 / 99$ & 1400 & $\mathrm{R}$ & -- & -- & -- & .79 & .34 & -- & -- & -- & -- \\
\hline 09/02/99 & 1445 & $\mathrm{R}$ & -- & -- & -- & .57 & .28 & -- & -- & -- & -- \\
\hline 09/27/99 & 1530 & $\mathrm{R}$ & -- & -- & -- & .34 & .14 & -- & -- & -- & -- \\
\hline $10 / 04 / 99$ & 1400 & $\mathrm{R}$ & -- & -- & -- & .34 & .14 & -- & -- & -- & -- \\
\hline
\end{tabular}


Table 5. Results of analysis of selected acetamide herbicides and degradation products by liquid chromatography/mass spectrometry methods HPAA (1998-99), LCAA (1999-02), and LCPD (2002-03) at the U.S. Geological Survey Organic Geochemistry Research Laboratory, Lawrence, Kansas, for water samples collected from the Mississippi River at Baton Rouge, Louisiana, 1998-2003.—Continued

[R, regular sample; L, laboratory duplicate sample; --, no data; <, less than]

\begin{tabular}{|c|c|c|c|c|c|c|c|c|c|c|c|}
\hline \multirow[b]{2}{*}{$\begin{array}{l}\text { Date of } \\
\text { collection } \\
\text { (month/ } \\
\text { day/year) }\end{array}$} & \multirow[b]{2}{*}{$\begin{array}{l}\text { Collection } \\
\text { time } \\
\text { (24-hour) }\end{array}$} & \multirow[b]{2}{*}{ Sample type } & \multicolumn{9}{|c|}{ Concentrations, in micrograms per liter } \\
\hline & & & $\begin{array}{l}\text { Flufenacet } \\
\text { ethane- } \\
\text { sulfonic } \\
\text { acid }\end{array}$ & $\begin{array}{c}\text { Flufenacet } \\
\text { oxanilic } \\
\text { acid }\end{array}$ & $\begin{array}{l}\text { Meto- } \\
\text { lachlor }\end{array}$ & $\begin{array}{l}\text { Metolachlor } \\
\text { ethane- } \\
\text { sulfonic acid }\end{array}$ & $\begin{array}{l}\text { Metolachlor } \\
\text { oxanilic acid }\end{array}$ & $\begin{array}{l}\text { Metolachlor/ } \\
\text { acetochlor } \\
\text { ethane- } \\
\text { sulfonic acid } \\
\text { 2nd amide }\end{array}$ & Propachlor & $\begin{array}{l}\text { Propachlor } \\
\text { ethane- } \\
\text { sulfonic } \\
\text { acid }\end{array}$ & $\begin{array}{l}\text { Propachlor } \\
\text { oxanilic acid }\end{array}$ \\
\hline $10 / 20 / 99$ & 1430 & $\mathrm{R}$ & -- & -- & -- & 0.26 & 0.13 & -- & -- & -- & -- \\
\hline $11 / 01 / 99$ & 1430 & $\mathrm{R}$ & -- & -- & -- & .20 & .09 & -- & -- & -- & -- \\
\hline $11 / 01 / 99$ & 1430 & $\mathrm{~L}$ & -- & -- & -- & .28 & .13 & -- & -- & -- & -- \\
\hline $11 / 19 / 99$ & 1700 & $\mathrm{R}$ & -- & -- & -- & .19 & .08 & -- & -- & -- & -- \\
\hline $12 / 06 / 99$ & 1330 & $\mathrm{R}$ & -- & -- & -- & .18 & .09 & -- & -- & -- & -- \\
\hline $12 / 06 / 99$ & 1330 & $\mathrm{~L}$ & -- & -- & -- & .19 & .07 & -- & -- & -- & -- \\
\hline 01/13/00 & 1500 & $\mathrm{R}$ & -- & -- & -- & $<.20$ & $<.20$ & -- & -- & -- & -- \\
\hline $02 / 14 / 00$ & 1545 & $\mathrm{R}$ & -- & -- & -- & .32 & $<.05$ & -- & -- & -- & -- \\
\hline $05 / 12 / 00$ & 1500 & $\mathrm{R}$ & -- & -- & -- & .27 & .16 & -- & -- & -- & -- \\
\hline $05 / 23 / 00$ & 1330 & $\mathrm{R}$ & -- & -- & -- & .27 & .11 & -- & -- & -- & -- \\
\hline 06/20/00 & 1000 & $\mathrm{R}$ & -- & -- & -- & .55 & .28 & -- & -- & -- & -- \\
\hline 06/29/00 & 1145 & $\mathrm{R}$ & -- & -- & -- & .54 & .30 & -- & -- & -- & -- \\
\hline 07/07/00 & 1400 & $\mathrm{R}$ & -- & -- & -- & .73 & .45 & -- & -- & -- & -- \\
\hline 07/18/00 & 1200 & $\mathrm{R}$ & -- & -- & -- & .88 & .50 & -- & -- & -- & -- \\
\hline 08/03/00 & 1125 & $\mathrm{R}$ & -- & -- & -- & .79 & .39 & -- & -- & -- & -- \\
\hline 08/11/00 & 1045 & $\mathrm{R}$ & -- & -- & -- & .61 & .30 & -- & -- & -- & -- \\
\hline 08/21/00 & 1100 & $\mathrm{R}$ & -- & -- & -- & .37 & .20 & -- & -- & -- & -- \\
\hline 08/29/00 & 1230 & $\mathrm{R}$ & -- & -- & -- & .34 & .19 & -- & -- & -- & -- \\
\hline 08/29/00 & 1230 & $\mathrm{~L}$ & -- & -- & -- & .34 & .20 & -- & -- & -- & -- \\
\hline 09/11/00 & 1045 & $\mathrm{R}$ & -- & -- & -- & .36 & .16 & -- & -- & -- & -- \\
\hline 09/27/00 & 1400 & $\mathrm{R}$ & -- & -- & -- & .24 & .13 & -- & -- & -- & -- \\
\hline $10 / 10 / 00$ & 1130 & $\mathrm{R}$ & -- & -- & -- & .26 & .11 & -- & -- & -- & -- \\
\hline $10 / 19 / 00$ & 1145 & $\mathrm{R}$ & -- & -- & -- & .20 & .08 & -- & -- & -- & -- \\
\hline $11 / 06 / 00$ & 1400 & $\mathrm{R}$ & -- & -- & -- & .28 & .12 & -- & -- & -- & -- \\
\hline $11 / 20 / 00$ & 1230 & $\mathrm{R}$ & -- & -- & -- & .22 & .11 & -- & -- & -- & -- \\
\hline
\end{tabular}


Table 5. Results of analysis of selected acetamide herbicides and degradation products by liquid chromatography/mass spectrometry methods HPAA (1998-99) LCAA (1999-02), and LCPD (2002-03) at the U.S. Geological Survey Organic Geochemistry Research Laboratory, Lawrence, Kansas, for water samples collected from the Mississippi River at Baton Rouge, Louisiana, 1998-2003.-Continued

$[\mathrm{R}$, regular sample; L, laboratory duplicate sample; --, no data; <, less than $]$

\begin{tabular}{|c|c|c|c|c|c|c|c|c|c|c|c|}
\hline \multirow[b]{2}{*}{$\begin{array}{l}\text { Date of } \\
\text { collection } \\
\text { (month/ } \\
\text { day/year) }\end{array}$} & \multirow[b]{2}{*}{$\begin{array}{l}\text { Collection } \\
\text { time } \\
\text { (24-hour) }\end{array}$} & \multirow[b]{2}{*}{ Sample type } & \multicolumn{9}{|c|}{ Concentrations, in micrograms per liter } \\
\hline & & & $\begin{array}{l}\text { Flufenacet } \\
\text { ethane- } \\
\text { sulfonic } \\
\text { acid }\end{array}$ & $\begin{array}{l}\text { Flufenacet } \\
\text { oxanilic } \\
\text { acid }\end{array}$ & $\begin{array}{l}\text { Meto- } \\
\text { lachlor }\end{array}$ & $\begin{array}{l}\text { Metolachlor } \\
\text { ethane- } \\
\text { sulfonic acid }\end{array}$ & $\begin{array}{l}\text { Metolachlor } \\
\text { oxanilic acid }\end{array}$ & $\begin{array}{l}\text { Metolachlor/ } \\
\text { acetochlor } \\
\text { ethane- } \\
\text { sulfonic acid } \\
\text { 2nd amide }\end{array}$ & Propachlor & $\begin{array}{l}\text { Propachlor } \\
\text { ethane- } \\
\text { sulfonic } \\
\text { acid }\end{array}$ & $\begin{array}{l}\text { Propachlor } \\
\text { oxanilic acid }\end{array}$ \\
\hline $12 / 04 / 00$ & 1330 & $\mathrm{R}$ & -- & -- & -- & 0.26 & 0.11 & -- & -- & -- & -- \\
\hline $12 / 04 / 00$ & 1330 & $\mathrm{~L}$ & -- & -- & -- & .23 & .13 & -- & -- & -- & -- \\
\hline $12 / 18 / 00$ & 1130 & $\mathrm{R}$ & -- & -- & -- & .34 & .09 & -- & -- & -- & -- \\
\hline 01/05/01 & 1000 & $\mathrm{R}$ & -- & -- & -- & .13 & .09 & -- & -- & -- & -- \\
\hline 01/05/01 & 1000 & $\mathrm{~L}$ & -- & -- & -- & .15 & .07 & -- & -- & -- & -- \\
\hline 02/08/01 & 1130 & $\mathrm{R}$ & -- & -- & -- & .19 & .08 & -- & -- & -- & -- \\
\hline $02 / 23 / 01$ & 1400 & $\mathrm{R}$ & -- & -- & -- & .26 & .11 & -- & -- & -- & -- \\
\hline 03/06/01 & 1100 & $\mathrm{R}$ & -- & -- & -- & .23 & .10 & -- & -- & -- & -- \\
\hline 03/23/01 & 1500 & $\mathrm{R}$ & -- & -- & -- & .24 & .11 & -- & -- & -- & -- \\
\hline 04/06/01 & 1430 & $\mathrm{R}$ & -- & -- & -- & .30 & .14 & -- & -- & -- & -- \\
\hline 05/17/01 & 1415 & $\mathrm{R}$ & -- & -- & -- & .53 & .19 & -- & -- & -- & -- \\
\hline $05 / 24 / 01$ & 1400 & $\mathrm{R}$ & -- & -- & -- & .53 & .22 & -- & -- & -- & -- \\
\hline $05 / 24 / 01$ & 1400 & $\mathrm{~L}$ & -- & -- & -- & .54 & .23 & -- & -- & -- & -- \\
\hline $05 / 31 / 01$ & 1400 & $\mathrm{R}$ & -- & -- & -- & .53 & .25 & -- & -- & -- & -- \\
\hline 06/08/01 & 1400 & $\mathrm{R}$ & -- & -- & -- & .37 & .21 & -- & -- & -- & -- \\
\hline 07/02/01 & 1200 & $\mathrm{R}$ & -- & -- & -- & .64 & .37 & -- & -- & -- & -- \\
\hline 07/16/01 & 1330 & $\mathrm{R}$ & -- & -- & -- & .57 & .26 & -- & -- & -- & -- \\
\hline 07/30/01 & 1430 & $\mathrm{R}$ & -- & -- & -- & .40 & .21 & -- & -- & -- & -- \\
\hline 07/30/01 & 1430 & $\mathrm{~L}$ & -- & -- & -- & .42 & .21 & -- & -- & -- & -- \\
\hline 08/13/01 & 1130 & $\mathrm{R}$ & -- & -- & -- & .37 & .21 & -- & -- & -- & -- \\
\hline $08 / 28 / 01$ & 1330 & $\mathrm{R}$ & -- & -- & -- & .10 & .13 & -- & -- & -- & -- \\
\hline 09/18/01 & 1100 & $\mathrm{R}$ & -- & -- & -- & .08 & .10 & -- & -- & -- & -- \\
\hline 10/01/01 & 1330 & $\mathrm{R}$ & -- & -- & -- & .22 & .10 & -- & -- & -- & -- \\
\hline $10 / 16 / 01$ & 1200 & $\mathrm{R}$ & -- & -- & -- & .22 & .12 & -- & -- & -- & -- \\
\hline $10 / 16 / 01$ & 1200 & $\mathrm{~L}$ & -- & -- & -- & .21 & .11 & -- & -- & -- & -- \\
\hline
\end{tabular}


Table 5. Results of analysis of selected acetamide herbicides and degradation products by liquid chromatography/mass spectrometry methods HPAA (1998-99), LCAA (1999-02), and LCPD (2002-03) at the U.S. Geological Survey Organic Geochemistry Research Laboratory, Lawrence, Kansas, for water samples collected from the Mississippi River at Baton Rouge, Louisiana, 1998-2003.—Continued

[R, regular sample; L, laboratory duplicate sample; --, no data; <, less than]

\begin{tabular}{|c|c|c|c|c|c|c|c|c|c|c|c|}
\hline \multirow[b]{2}{*}{$\begin{array}{l}\text { Date of } \\
\text { collection } \\
\text { (month/ } \\
\text { day/year) }\end{array}$} & \multirow[b]{2}{*}{$\begin{array}{l}\text { Collection } \\
\text { time } \\
\text { (24-hour) }\end{array}$} & \multirow[b]{2}{*}{ Sample type } & \multicolumn{9}{|c|}{ Concentrations, in micrograms per liter } \\
\hline & & & $\begin{array}{l}\text { Flufenacet } \\
\text { ethane- } \\
\text { sulfonic } \\
\text { acid }\end{array}$ & $\begin{array}{l}\text { Flufenacet } \\
\text { oxanilic } \\
\text { acid }\end{array}$ & $\begin{array}{l}\text { Meto- } \\
\text { lachlor }\end{array}$ & $\begin{array}{l}\text { Metolachlor } \\
\text { ethane- } \\
\text { sulfonic acid }\end{array}$ & $\begin{array}{l}\text { Metolachlor } \\
\text { oxanilic acid }\end{array}$ & $\begin{array}{l}\text { Metolachlor/ } \\
\text { acetochlor } \\
\text { ethane- } \\
\text { sulfonic acid } \\
\text { 2nd amide }\end{array}$ & Propachlor & $\begin{array}{l}\text { Propachlor } \\
\text { ethane- } \\
\text { sulfonic } \\
\text { acid }\end{array}$ & $\begin{array}{l}\text { Propachlor } \\
\text { oxanilic acid }\end{array}$ \\
\hline $10 / 31 / 01$ & 1300 & $\mathrm{R}$ & -- & -- & -- & 0.23 & 0.13 & -- & -- & -- & -- \\
\hline $11 / 14 / 01$ & 1300 & $\mathrm{R}$ & -- & -- & -- & .43 & .17 & -- & -- & -- & -- \\
\hline $11 / 14 / 01$ & 1300 & $\mathrm{~L}$ & -- & -- & -- & .40 & .16 & -- & -- & -- & -- \\
\hline $11 / 28 / 01$ & 1200 & $\mathrm{R}$ & -- & -- & -- & .51 & .19 & -- & -- & -- & -- \\
\hline $12 / 10 / 01$ & 1300 & $\mathrm{R}$ & -- & -- & -- & .19 & .11 & -- & -- & $<0.05$ & -- \\
\hline 01/03/02 & 1140 & $\mathrm{R}$ & -- & -- & -- & .18 & .09 & -- & -- & $<.05$ & -- \\
\hline 01/03/02 & 1140 & $\mathrm{~L}$ & -- & -- & -- & .18 & .08 & -- & -- & $<.05$ & -- \\
\hline 01/17/02 & 1100 & $\mathrm{R}$ & -- & -- & -- & .22 & .08 & -- & -- & $<.05$ & -- \\
\hline 02/04/02 & 1300 & $\mathrm{R}$ & -- & -- & -- & .14 & .07 & -- & -- & $<.05$ & -- \\
\hline 02/28/02 & 1230 & $\mathrm{R}$ & -- & -- & -- & .24 & .07 & -- & -- & $<.05$ & -- \\
\hline $02 / 28 / 02$ & 1230 & $\mathrm{~L}$ & -- & -- & -- & .24 & .10 & -- & -- & $<.05$ & -- \\
\hline 03/11/02 & 1300 & $\mathrm{R}$ & -- & -- & -- & .25 & $<.05$ & -- & -- & $<.05$ & -- \\
\hline 03/28/02 & 1300 & $\mathrm{R}$ & -- & -- & -- & .22 & .09 & -- & -- & $<.05$ & -- \\
\hline 03/28/02 & 1300 & $\mathrm{~L}$ & -- & -- & -- & .23 & .08 & -- & -- & $<.05$ & -- \\
\hline $04 / 12 / 02$ & 1115 & $\mathrm{R}$ & -- & -- & -- & .14 & .06 & -- & -- & $<.05$ & -- \\
\hline 04/12/02 & 1115 & $\mathrm{~L}$ & -- & -- & -- & .13 & .06 & -- & -- & $<.05$ & -- \\
\hline 05/02/02 & 1300 & $\mathrm{R}$ & -- & -- & -- & .32 & .11 & -- & -- & $<.05$ & -- \\
\hline 05/15/02 & 1300 & $\mathrm{R}$ & -- & -- & -- & .26 & .11 & -- & -- & $<.05$ & -- \\
\hline 05/15/02 & 1300 & $\mathrm{~L}$ & -- & -- & -- & .25 & .12 & -- & -- & $<.05$ & -- \\
\hline 06/05/02 & 1200 & $\mathrm{R}$ & -- & -- & -- & .36 & .16 & -- & -- & $<.05$ & -- \\
\hline 06/05/02 & 1200 & $\mathrm{~L}$ & -- & -- & -- & .35 & .16 & -- & -- & $<.05$ & -- \\
\hline 06/11/02 & 1030 & $\mathrm{R}$ & -- & -- & -- & .28 & .17 & -- & -- & $<.05$ & -- \\
\hline 07/02/02 & 1130 & $\mathrm{R}$ & -- & -- & -- & .39 & .24 & -- & -- & $<.05$ & -- \\
\hline 07/02/02 & 1130 & $\mathrm{~L}$ & -- & -- & -- & .43 & .26 & -- & -- & $<.05$ & -- \\
\hline 07/25/02 & 1400 & $\mathrm{R}$ & -- & -- & -- & .29 & .08 & -- & -- & $<.05$ & -- \\
\hline
\end{tabular}


Table 5. Results of analysis of selected acetamide herbicides and degradation products by liquid chromatography/mass spectrometry methods HPAA (1998-99) LCAA (1999-02), and LCPD (2002-03) at the U.S. Geological Survey Organic Geochemistry Research Laboratory, Lawrence, Kansas, for water samples collected from the Mississippi River at Baton Rouge, Louisiana, 1998-2003.-Continued

[R, regular sample; L, laboratory duplicate sample; --, no data; <, less than]

\begin{tabular}{|c|c|c|c|c|c|c|c|c|c|c|c|}
\hline \multirow[b]{2}{*}{$\begin{array}{l}\text { Date of } \\
\text { collection } \\
\text { (month/ } \\
\text { day/year) }\end{array}$} & \multirow[b]{2}{*}{$\begin{array}{l}\text { Collection } \\
\text { time } \\
\text { (24-hour) }\end{array}$} & \multirow[b]{2}{*}{ Sample type } & \multicolumn{9}{|c|}{ Concentrations, in micrograms per liter } \\
\hline & & & $\begin{array}{l}\text { Flufenacet } \\
\text { ethane- } \\
\text { sulfonic } \\
\text { acid }\end{array}$ & $\begin{array}{c}\text { Flufenacet } \\
\text { oxanilic } \\
\text { acid }\end{array}$ & $\begin{array}{l}\text { Meto- } \\
\text { lachlor }\end{array}$ & $\begin{array}{l}\text { Metolachlor } \\
\text { ethane- } \\
\text { sulfonic acid }\end{array}$ & $\begin{array}{l}\text { Metolachlor } \\
\text { oxanilic acid }\end{array}$ & $\begin{array}{l}\text { Metolachlor/ } \\
\text { acetochlor } \\
\text { ethane- } \\
\text { sulfonic acid } \\
\text { 2nd amide }\end{array}$ & Propachlor & $\begin{array}{l}\text { Propachlor } \\
\text { ethane- } \\
\text { sulfonic } \\
\text { acid }\end{array}$ & $\begin{array}{l}\text { Propachlor } \\
\text { oxanilic acid }\end{array}$ \\
\hline $08 / 21 / 02$ & 1100 & $\mathrm{R}$ & -- & -- & -- & 0.19 & 0.09 & -- & -- & $<0.05$ & -- \\
\hline 09/17/02 & 1400 & $\mathrm{R}$ & -- & -- & -- & .24 & .11 & -- & -- & $<.05$ & -- \\
\hline $10 / 16 / 02$ & 1500 & $\mathrm{R}$ & -- & -- & -- & .11 & .08 & -- & -- & $<.05$ & -- \\
\hline $11 / 19 / 02$ & 1400 & $\mathrm{R}$ & -- & -- & -- & .17 & .05 & -- & -- & $<.05$ & -- \\
\hline $12 / 18 / 02$ & 1330 & $\mathrm{R}$ & $<0.02$ & $<0.02$ & 0.03 & .08 & .04 & $<0.02$ & $<0.02$ & $<.05$ & $<0.02$ \\
\hline $01 / 15 / 03$ & 1400 & $\mathrm{R}$ & $<.02$ & $<.02$ & $<.02$ & .13 & .08 & $<.02$ & $<.02$ & $<.05$ & $<.02$ \\
\hline $02 / 20 / 03$ & 1330 & $\mathrm{R}$ & $<.02$ & $<.02$ & .03 & .11 & .06 & $<.02$ & $<.02$ & $<.05$ & $<.02$ \\
\hline 03/13/03 & 1200 & $\mathrm{R}$ & $<.02$ & $<.02$ & $<.02$ & .06 & .03 & $<.02$ & $<.02$ & $<.05$ & $<.02$ \\
\hline 04/03/03 & 1100 & $\mathrm{R}$ & $<.02$ & $<.02$ & .04 & .15 & .06 & $<.02$ & $<.02$ & $<.05$ & $<.02$ \\
\hline 05/19/03 & 1330 & $\mathrm{R}$ & $<.02$ & $<.02$ & .38 & .17 & .11 & .02 & $<.02$ & $<.05$ & $<.02$ \\
\hline 06/03/03 & 1230 & $\mathrm{R}$ & $<.02$ & $<.02$ & .34 & .20 & .13 & .04 & $<.02$ & $<.05$ & $<.02$ \\
\hline 06/19/03 & 0830 & $\mathrm{R}$ & $<.02$ & $<.02$ & .17 & .23 & .10 & .02 & $<.02$ & $<.05$ & $<.02$ \\
\hline 07/03/03 & 1330 & $\mathrm{R}$ & $<.02$ & $<.02$ & .24 & .28 & .20 & .06 & $<.02$ & $<.05$ & $<.02$ \\
\hline $07 / 15 / 03$ & 1230 & $\mathrm{R}$ & $<.02$ & $<.02$ & .13 & .23 & .12 & .04 & $<.02$ & $<.05$ & $<.02$ \\
\hline 07/30/03 & 1130 & $\mathrm{R}$ & $<.02$ & $<.02$ & .16 & .45 & .25 & .09 & $<.02$ & $<.05$ & $<.02$ \\
\hline 09/17/03 & 1400 & $\mathrm{R}$ & $<.02$ & $<.02$ & .02 & .11 & .07 & $<.02$ & $<.02$ & .06 & $<.02$ \\
\hline $09 / 29 / 03$ & 1300 & $\mathrm{R}$ & $<.02$ & $<.02$ & .03 & .25 & .14 & .05 & $<.02$ & $<.05$ & $<.02$ \\
\hline $10 / 15 / 03$ & 1430 & $\mathrm{R}$ & $<.02$ & $<.02$ & .02 & .12 & .07 & .03 & $<.02$ & $<.05$ & $<.02$ \\
\hline $11 / 05 / 03$ & 1230 & $\mathrm{R}$ & $<.02$ & $<.02$ & .02 & .16 & .09 & .03 & $<.02$ & $<.05$ & $<.02$ \\
\hline $12 / 01 / 03$ & 1055 & $\mathrm{R}$ & $<.02$ & $<.02$ & $<.02$ & $<.02$ & $<.02$ & $<.02$ & $<.02$ & $<.05$ & $<.02$ \\
\hline $12 / 01 / 03$ & 1100 & $\mathrm{R}$ & $<.02$ & $<.02$ & .02 & .10 & .06 & $<.02$ & $<.02$ & $<.05$ & $<.02$ \\
\hline $12 / 10 / 03$ & 1100 & $\mathrm{~L}$ & $<.02$ & $<.02$ & .02 & .15 & .07 & .03 & $<.02$ & $<.05$ & $<.02$ \\
\hline $12 / 30 / 03$ & 1030 & $\mathrm{R}$ & $<.02$ & $<.02$ & $<.02$ & .12 & .07 & $<.02$ & $<.02$ & $<.05$ & $<.02$ \\
\hline
\end{tabular}


Table 6. Results of analysis of selected triazine and phenylurea herbicides and their degradation products analyzed by liquid chromatography/mass spectrometry method LCEA at the U.S. Geological Survey Organic Geochemistry Research Laboratory, Lawrence, Kansas, for water samples collected from the Mississippi River at Baton Rouge, Louisiana, 1998-2003.

$[R$, regular sample; L, laboratory duplicate sample; <, less than; bromacil, cyanazine amide, deethylcyanazine, deethylcyanazine amide, demethylfluometuron, deethylhydroxyatrazine, fluometuron, hydroxysimazine, linuron, and propazine were not detected]

\begin{tabular}{|c|c|c|c|c|c|c|c|c|c|c|c|c|c|}
\hline \multirow[b]{2}{*}{$\begin{array}{c}\text { Date of } \\
\text { collection } \\
\text { (month/ } \\
\text { day/year) }\end{array}$} & \multirow[b]{2}{*}{$\begin{array}{l}\text { Collection } \\
\text { time } \\
\text { (24-hour) }\end{array}$} & \multirow[b]{2}{*}{$\begin{array}{c}\text { Sample } \\
\text { type }\end{array}$} & \multicolumn{11}{|c|}{ Concentrations, in micrograms per liter } \\
\hline & & & Atrazine & Cyanazine & $\begin{array}{c}\text { Cyanazine } \\
\text { acid }\end{array}$ & $\begin{array}{l}\text { Deethyl- } \\
\text { atrazine }\end{array}$ & $\begin{array}{l}\text { Deethyl- } \\
\text { cyanazine } \\
\text { acid }\end{array}$ & $\begin{array}{l}\text { Deiso- } \\
\text { propyl- } \\
\text { atrazine }\end{array}$ & $\begin{array}{l}\text { Deiso- } \\
\text { propyl- } \\
\text { hydroxy- } \\
\text { atrazine }\end{array}$ & $\begin{array}{c}\text { Dide- } \\
\text { alkyl- } \\
\text { atrazine }\end{array}$ & Diuron & $\begin{array}{l}\text { Hydroxy- } \\
\text { atrazine }\end{array}$ & Simazine \\
\hline $08 / 27 / 98$ & 1120 & $\mathrm{R}$ & 0.32 & 0.07 & 0.16 & 0.12 & 0.37 & 0.09 & $<0.025$ & 0.07 & $<0.20$ & 0.21 & $<0.025$ \\
\hline $10 / 30 / 98$ & 1330 & $\mathrm{R}$ & .24 & $<.025$ & $<.025$ & $<.025$ & $<.025$ & $<.025$ & $<.025$ & .04 & $<.20$ & .13 & $<.025$ \\
\hline $12 / 23 / 98$ & 0745 & $\mathrm{R}$ & .08 & $<.025$ & $<.025$ & .03 & $<.025$ & $<.025$ & $<.025$ & .04 & $<.20$ & .06 & $<.025$ \\
\hline $12 / 23 / 98$ & 0745 & $\mathrm{~L}$ & .10 & $<.025$ & $<.025$ & .05 & $<.025$ & $<.025$ & $<.025$ & .05 & $<.20$ & .09 & $<.025$ \\
\hline $01 / 05 / 99$ & 1100 & $\mathrm{R}$ & .37 & $<.025$ & $<.025$ & .31 & $<.025$ & $<.025$ & $<.025$ & $<.025$ & $<.20$ & $<.025$ & $<.025$ \\
\hline 08/03/00 & 1125 & $\mathrm{R}$ & .29 & $<.025$ & $<.025$ & .10 & $<.025$ & $<.025$ & $<.025$ & $<.025$ & $<.20$ & .25 & $<.025$ \\
\hline 08/11/00 & 1045 & $\mathrm{R}$ & .14 & $<.025$ & $<.025$ & .05 & $<.025$ & $<.025$ & $<.025$ & $<.025$ & $<.20$ & .12 & $<.025$ \\
\hline $08 / 21 / 00$ & 1100 & $\mathrm{R}$ & .12 & $<.025$ & $<.025$ & .05 & $<.025$ & $<.025$ & $<.025$ & $<.025$ & $<.20$ & .14 & $<.025$ \\
\hline $11 / 06 / 00$ & 1400 & $\mathrm{R}$ & .07 & $<.025$ & .04 & .04 & $<.025$ & $<.025$ & $<.025$ & .07 & $<.20$ & .08 & $<.025$ \\
\hline $11 / 06 / 00$ & 1400 & $\mathrm{~L}$ & .14 & $<.025$ & $<.025$ & .07 & $<.025$ & $<.025$ & $<.025$ & .06 & $<.20$ & .12 & $<.025$ \\
\hline $12 / 18 / 00$ & 1130 & $\mathrm{R}$ & .14 & $<.025$ & $<.025$ & .06 & $<.025$ & $<.025$ & $<.025$ & $<.025$ & $<.20$ & .08 & .12 \\
\hline $12 / 18 / 00$ & 1130 & $\mathrm{~L}$ & .11 & $<.025$ & $<.025$ & .04 & $<.025$ & $<.025$ & $<.025$ & $<.025$ & $<.20$ & .11 & .10 \\
\hline 05/17/01 & 1415 & $\mathrm{R}$ & .22 & $<.025$ & $<.025$ & .05 & $<.025$ & $<.025$ & $<.025$ & .10 & $<.20$ & .08 & $<.025$ \\
\hline $05 / 24 / 01$ & 1400 & $\mathrm{R}$ & .39 & $<.025$ & $<.025$ & .25 & .37 & .16 & $<.025$ & .13 & $<.20$ & .13 & .04 \\
\hline $05 / 31 / 01$ & 1400 & $\mathrm{R}$ & .42 & $<.025$ & $<.025$ & .18 & $<.025$ & .13 & $<.025$ & .07 & $<.20$ & .22 & .05 \\
\hline 06/08/01 & 1400 & $\mathrm{R}$ & .42 & $<.025$ & $<.025$ & .18 & $<.025$ & .17 & $<.025$ & $<.025$ & $<.20$ & .12 & .06 \\
\hline $07 / 02 / 01$ & 1200 & $\mathrm{R}$ & .48 & $<.025$ & $<.025$ & .27 & $<.025$ & .14 & $<.025$ & .08 & $<.20$ & .27 & .03 \\
\hline 07/02/01 & 1200 & $\mathrm{~L}$ & .42 & $<.025$ & $<.025$ & .25 & $<.025$ & .17 & $<.025$ & .17 & $<.20$ & .20 & .03 \\
\hline $07 / 16 / 01$ & 1330 & $\mathrm{R}$ & .89 & $<.025$ & $<.025$ & .24 & $<.025$ & .15 & $<.025$ & .13 & $<.20$ & .22 & .04 \\
\hline 07/30/01 & 1430 & $\mathrm{R}$ & .53 & $<.025$ & $<.025$ & .14 & $<.025$ & $<.025$ & $<.025$ & .10 & .14 & .19 & $<.025$ \\
\hline
\end{tabular}


Table 6. Results of analysis of selected triazine and phenylurea herbicides and their degradation products analyzed by liquid chromatography/mass spectrometry method LCEA at the U.S. Geological Survey Organic Geochemistry Research Laboratory, Lawrence, Kansas, for water samples collected from the Mississippi River at Baton Rouge, Louisiana, 1998-2003.—Continued

[R, regular sample; L, laboratory duplicate sample; <, less than; bromacil, cyanazine amide, deethylcyanazine, deethylcyanazine amide, demethylfluometuron, deethylhydroxyatrazine, fluometuron, hydroxysimazine, linuron, and propazine were not detected]

\begin{tabular}{|c|c|c|c|c|c|c|c|c|c|c|c|c|c|}
\hline \multirow[b]{2}{*}{$\begin{array}{c}\text { Date of } \\
\text { collection } \\
\text { (month/ } \\
\text { day/year) }\end{array}$} & \multirow[b]{2}{*}{$\begin{array}{l}\text { Collection } \\
\text { time } \\
\text { (24-hour) }\end{array}$} & \multirow[b]{2}{*}{$\begin{array}{l}\text { Sample } \\
\text { type }\end{array}$} & \multicolumn{11}{|c|}{ Concentrations, in micrograms per liter } \\
\hline & & & Atrazine & Cyanazine & $\begin{array}{c}\text { Cyanazine } \\
\text { acid }\end{array}$ & $\begin{array}{l}\text { Deethyl- } \\
\text { atrazine }\end{array}$ & $\begin{array}{c}\text { Deethyl- } \\
\text { cyanazine } \\
\text { acid }\end{array}$ & $\begin{array}{l}\text { Deiso- } \\
\text { propyl- } \\
\text { atrazine }\end{array}$ & $\begin{array}{l}\text { Deiso- } \\
\text { propyl- } \\
\text { hydroxy- } \\
\text { atrazine }\end{array}$ & $\begin{array}{l}\text { Dide- } \\
\text { alkyl- } \\
\text { atrazine }\end{array}$ & Diuron & $\begin{array}{l}\text { Hydroxy- } \\
\text { atrazine }\end{array}$ & Simazine \\
\hline $08 / 13 / 01$ & 1130 & $\mathrm{R}$ & 0.33 & $<0.025$ & $<0.025$ & 0.13 & $<0.025$ & 0.08 & $<0.025$ & 0.09 & 0.07 & 0.16 & $<0.025$ \\
\hline $11 / 14 / 01$ & 1300 & $\mathrm{R}$ & .07 & $<.025$ & $<.025$ & .06 & $<.025$ & .06 & $<.025$ & .07 & $<.20$ & .11 & .04 \\
\hline $05 / 02 / 02$ & 1300 & $\mathrm{R}$ & .45 & $<.025$ & $<.025$ & .05 & $<.025$ & $<.025$ & .04 & $<.025$ & $<.20$ & .07 & .12 \\
\hline $06 / 05 / 02$ & 1200 & $\mathrm{R}$ & .27 & $<.025$ & $<.025$ & .21 & $<.025$ & .13 & $<.025$ & $<.025$ & .14 & $<.025$ & .07 \\
\hline $07 / 02 / 02$ & 1130 & $\mathrm{R}$ & .26 & $<.025$ & $<.025$ & .37 & $<.025$ & .21 & $<.025$ & .12 & $<.20$ & .18 & .03 \\
\hline 06/03/03 & 1230 & $\mathrm{R}$ & 1.3 & $<.025$ & $<.025$ & .20 & $<.025$ & .10 & $<.025$ & $<.025$ & $<.20$ & .13 & .11 \\
\hline 06/19/03 & 0830 & $\mathrm{R}$ & 1.1 & $<.025$ & $<.025$ & .19 & $<.025$ & .11 & $<.025$ & .10 & $<.20$ & .18 & .10 \\
\hline 07/03/03 & 1330 & $\mathrm{R}$ & .60 & $<.025$ & $<.025$ & .22 & $<.025$ & .11 & $<.025$ & .11 & $<.20$ & .11 & .08 \\
\hline $07 / 15 / 03$ & 1230 & $\mathrm{R}$ & .72 & $<.025$ & $<.025$ & .16 & $<.025$ & .09 & $<.025$ & .09 & $<.20$ & .14 & .04 \\
\hline 07/30/03 & 1130 & $\mathrm{R}$ & .61 & $<.025$ & $<.025$ & .22 & $<.025$ & .14 & $<.025$ & .21 & $<.20$ & .24 & .04 \\
\hline 09/17/03 & 1400 & $\mathrm{R}$ & .13 & $<.025$ & $<.025$ & .05 & $<.025$ & $<.025$ & $<.025$ & $<.025$ & $<.20$ & .08 & $<.025$ \\
\hline 09/29/03 & 1300 & $\mathrm{R}$ & .16 & $<.025$ & $<.025$ & .08 & $<.025$ & .05 & $<.025$ & .08 & $<.20$ & .14 & $<.025$ \\
\hline $10 / 15 / 03$ & 1430 & $\mathrm{R}$ & .12 & $<.025$ & $<.025$ & .07 & $<.025$ & .03 & $<.025$ & .06 & $<.20$ & .10 & $<.025$ \\
\hline $11 / 05 / 03$ & 1230 & $\mathrm{R}$ & .09 & $<.025$ & $<.025$ & .05 & $<.025$ & .03 & $<.025$ & .04 & $<.20$ & .13 & .03 \\
\hline $12 / 01 / 03$ & 1055 & B & $<.025$ & $<.025$ & $<.025$ & $<.025$ & $<.025$ & $<.025$ & $<.025$ & $<.025$ & $<.20$ & $<.025$ & $<.025$ \\
\hline $12 / 01 / 03$ & 1100 & $\mathrm{R}$ & .11 & $<.025$ & $<.025$ & $<.025$ & $<.025$ & .03 & $<.025$ & .03 & $<.20$ & .09 & .11 \\
\hline $12 / 10 / 03$ & 1100 & $\mathrm{R}$ & .07 & $<.025$ & $<.025$ & .04 & $<.025$ & .06 & $<.025$ & $<.025$ & $<.20$ & .05 & .26 \\
\hline $12 / 30 / 03$ & 1030 & $\mathrm{R}$ & .08 & $<.025$ & $<.025$ & .04 & $<.025$ & .03 & $<.025$ & $<.025$ & $<.20$ & $<.025$ & .08 \\
\hline
\end{tabular}


Table 7. Results of analysis of glyphosate, its degradation product aminomethylphosphonic acid, and glufosinate by liquid chromatography/mass spectrometry method LCGY at the U.S. Geological Survey Organic Geochemistry Laboratory, Lawrence, Kansas, for water samples collected from the Mississippi River at Baton Rouge, Louisiana, 2001-03.

[R, regular sample; L, laboratory duplicate sample; <, less than]

\begin{tabular}{|c|c|c|c|c|c|}
\hline \multirow{2}{*}{$\begin{array}{c}\text { Date of } \\
\text { collection } \\
\text { (month/day/ } \\
\text { year) }\end{array}$} & \multirow{2}{*}{$\begin{array}{l}\text { Collection } \\
\text { time } \\
\text { (24-hour) }\end{array}$} & \multirow{2}{*}{$\begin{array}{l}\text { Sample } \\
\text { type }\end{array}$} & \multicolumn{3}{|c|}{ Concentrations, in micrograms per liter } \\
\hline & & & Glyphosate & $\begin{array}{l}\text { Aminomethyl- } \\
\text { phosphonic acid }\end{array}$ & Glufosinate \\
\hline $10 / 31 / 01$ & 1300 & $\mathrm{R}$ & $<0.10$ & 0.18 & $<0.10$ \\
\hline $11 / 14 / 01$ & 1300 & $\mathrm{R}$ & $<.10$ & .18 & $<.10$ \\
\hline $11 / 28 / 01$ & 1200 & $\mathrm{R}$ & $<.10$ & .23 & $<.10$ \\
\hline $12 / 10 / 01$ & 1300 & $\mathrm{R}$ & $<.10$ & .14 & $<.10$ \\
\hline $12 / 10 / 01$ & 1300 & $\mathrm{~L}$ & $<.10$ & .11 & $<.10$ \\
\hline 01/03/02 & 1140 & $\mathrm{R}$ & $<.10$ & .15 & $<.10$ \\
\hline 01/03/02 & 1140 & $\mathrm{~L}$ & $<.10$ & .10 & $<.10$ \\
\hline 01/17/02 & 1100 & $\mathrm{R}$ & $<.10$ & .12 & $<.10$ \\
\hline 01/17/02 & 1100 & $\mathrm{~L}$ & $<.10$ & .13 & $<.10$ \\
\hline 02/04/02 & 1300 & $\mathrm{R}$ & $<.10$ & .15 & $<.10$ \\
\hline 02/04/02 & 1300 & $\mathrm{~L}$ & $<.10$ & .14 & $<.10$ \\
\hline $02 / 28 / 02$ & 1230 & $\mathrm{R}$ & $<.10$ & .15 & $<.10$ \\
\hline $02 / 28 / 02$ & 1230 & $\mathrm{~L}$ & $<.10$ & .18 & $<.10$ \\
\hline $03 / 11 / 02$ & 1300 & $\mathrm{R}$ & $<.10$ & .24 & $<.10$ \\
\hline 03/28/02 & 1300 & $\mathrm{R}$ & $<.10$ & .38 & $<.10$ \\
\hline $04 / 12 / 02$ & 1115 & $\mathrm{R}$ & $<.10$ & $<.10$ & $<.10$ \\
\hline $05 / 02 / 02$ & 1300 & $\mathrm{R}$ & $<.10$ & $<.10$ & $<.10$ \\
\hline 05/15/02 & 1300 & $\mathrm{R}$ & $<.10$ & .12 & $<.10$ \\
\hline 06/05/02 & 1200 & $\mathrm{R}$ & $<.10$ & .16 & $<.10$ \\
\hline $06 / 11 / 02$ & 1030 & $\mathrm{R}$ & $<.10$ & .16 & $<.10$ \\
\hline $07 / 02 / 02$ & 1130 & $\mathrm{R}$ & $<.10$ & .17 & $<.10$ \\
\hline $07 / 25 / 02$ & 1400 & $\mathrm{R}$ & $<.10$ & .21 & $<.10$ \\
\hline 08/21/02 & 1100 & $\mathrm{R}$ & $<.10$ & .21 & $<.10$ \\
\hline 09/17/02 & 1400 & $\mathrm{R}$ & $<.10$ & .27 & $<.10$ \\
\hline 09/17/02 & 1400 & $\mathrm{~L}$ & $<.10$ & .22 & $<.10$ \\
\hline $10 / 16 / 02$ & 1500 & $\mathrm{R}$ & $<.10$ & .24 & $<.10$ \\
\hline $11 / 19 / 02$ & 1400 & $\mathrm{R}$ & $<.10$ & .12 & $<.10$ \\
\hline $11 / 19 / 02$ & 1400 & $\mathrm{~L}$ & $<.10$ & .13 & $<.10$ \\
\hline $12 / 18 / 02$ & 1330 & $\mathrm{R}$ & $<.10$ & .10 & $<.10$ \\
\hline $01 / 15 / 03$ & 1400 & $\mathrm{R}$ & $<.10$ & $<.10$ & $<.10$ \\
\hline $02 / 20 / 03$ & 1330 & $\mathrm{R}$ & $<.10$ & .12 & $<.10$ \\
\hline $02 / 20 / 03$ & 1330 & $\mathrm{~L}$ & $<.10$ & .17 & $<.10$ \\
\hline $03 / 13 / 03$ & 1200 & $\mathrm{R}$ & $<.10$ & .11 & $<.10$ \\
\hline $04 / 03 / 03$ & 1100 & $\mathrm{R}$ & $<.10$ & .20 & $<.10$ \\
\hline 05/19/03 & 1330 & $\mathrm{R}$ & $<.10$ & .18 & $<.10$ \\
\hline
\end{tabular}


Table 7. Results of analysis of glyphosate, its degradation product aminomethylphosphonic acid, and glufosinate by liquid chromatography/mass spectrometry method LCGY at the U.S. Geological Survey Organic Geochemistry Laboratory, Lawrence, Kansas, for water samples collected from the Mississippi River at Baton Rouge, Louisiana, 2001-03.—Continued

[R, regular sample; L, laboratory duplicate sample; <, less than]

\begin{tabular}{cccccc}
\hline \multirow{2}{*}{$\begin{array}{c}\text { Date of } \\
\text { collection } \\
\text { (month/day/ }\end{array}$} & $\begin{array}{c}\text { Collection } \\
\text { time } \\
\text { year) }\end{array}$ & Sample & \multicolumn{3}{c}{ Concentrations, in micrograms per liter } \\
\cline { 5 - 6 } & type & Glyphosate & $\begin{array}{c}\text { Aminomethyl- } \\
\text { phosphonic acid }\end{array}$ & Glufosinate \\
\hline $05 / 19 / 03$ & 1330 & $\mathrm{R}$ & $<0.10$ & 0.20 & $<0.10$ \\
$06 / 03 / 03$ & 1230 & $\mathrm{R}$ & $<.10$ & .19 & $<.10$ \\
$06 / 19 / 03$ & 0830 & $\mathrm{R}$ & $<.10$ & .20 & $<.10$ \\
$07 / 03 / 03$ & 1330 & $\mathrm{R}$ & $<.10$ & $<.10$ & $<.10$ \\
$07 / 15 / 03$ & 1230 & $\mathrm{R}$ & $<.10$ & .31 & $<.10$ \\
& & & & & \\
$07 / 30 / 03$ & 1130 & $\mathrm{R}$ & $<.10$ & .31 & $<.10$ \\
$9 / 17 / 03$ & 1400 & $\mathrm{R}$ & $<.10$ & .30 & $<.10$ \\
$9 / 29 / 03$ & 1300 & $\mathrm{R}$ & $<.10$ & .33 & $<.10$ \\
$10 / 15 / 03$ & 1430 & $\mathrm{R}$ & $<.10$ & .27 & $<.10$ \\
\hline
\end{tabular}


Table 8. Results of analysis of streamflow, physical properties, and nutrients for water samples collected from three sampling sites in the lower Mississippi River, Louisiana, 1991-2003.

[Streamflow data from U.S. Army Corps of Engineers, New Orleans District. Physical property data from various collectors. Nutrient data from U.S. Geological Survey National Water-Quality Laboratory, Lakewood, Colorado; $\mathrm{ft}^{3} / \mathrm{s}$, cubic feet per second; $\mu \mathrm{S} / \mathrm{cm}$, microsiemens per centimeter at 25 degrees Celsius; ${ }^{\circ} \mathrm{C}$, degrees Celsius; $\mathrm{mg} / \mathrm{L}$, milligrams per liter; <, less than; --, no data]

\begin{tabular}{|c|c|c|c|c|c|c|c|c|}
\hline $\begin{array}{l}\text { Collection } \\
\text { (month/day/ } \\
\text { year) }\end{array}$ & $\begin{array}{c}\text { Streamflow } \\
\left(\mathrm{ft}^{3} / \mathrm{s}\right)\end{array}$ & $\begin{array}{c}\text { Specific } \\
\text { conductance } \\
(\mu \mathrm{S} / \mathrm{cm})\end{array}$ & $\begin{array}{c}\text { Water } \\
\text { temperature } \\
\left({ }^{\circ} \mathrm{C}\right)\end{array}$ & $\begin{array}{c}\text { Nitrite as } \\
\text { nitrogen } \\
\text { (mg/L) }\end{array}$ & $\begin{array}{l}\text { Nitrite plus } \\
\text { nitrate as } \\
\text { nitrogen } \\
\text { (mg/L) }\end{array}$ & $\begin{array}{l}\text { Ammonia } \\
\text { as nitrogen } \\
\text { (mg/L) }\end{array}$ & $\begin{array}{c}\text { Orthophosphate } \\
\text { as phosphorus } \\
\text { (mg/L) }\end{array}$ & $\begin{array}{c}\text { Silica as } \\
\mathrm{SiO}_{2} \text { (mg/L) }\end{array}$ \\
\hline \multicolumn{9}{|c|}{ Mississippi River near St. Francisville, Louisiana, station 07373420 (fig. 1) } \\
\hline $04 / 02 / 91$ & $1,008,000$ & 322 & 22.0 & 0.03 & 1.5 & 0.01 & 0.04 & 6.9 \\
\hline 05/06/91 & $1,380,000$ & 274 & 19.0 & $<.01$ & 1.6 & $<.01$ & .06 & -- \\
\hline 06/17/91 & 826,000 & 389 & 27.5 & $<.01$ & 2.6 & .02 & .08 & 7.3 \\
\hline $06 / 17 / 91$ & 826,000 & 387 & 27.5 & $<.01$ & 2.5 & .02 & .08 & -- \\
\hline $07 / 23 / 91$ & 457,000 & -- & -- & $<.01$ & 2.0 & .01 & .08 & -- \\
\hline $09 / 23 / 91$ & 258,000 & 433 & 27.0 & $<.01$ & .55 & .03 & .06 & 2.6 \\
\hline $11 / 05 / 91$ & 362,000 & 560 & 16.0 & $<.01$ & .64 & .04 & .06 & 5.1 \\
\hline $11 / 05 / 91$ & 362,000 & 560 & 16.0 & $<.01$ & .68 & .05 & .05 & -- \\
\hline $12 / 10 / 91$ & 794,000 & 321 & 8.0 & .03 & 1.3 & .05 & .06 & 6.6 \\
\hline $01 / 28 / 92$ & 578,000 & 348 & 5.0 & .03 & 1.7 & .04 & .06 & -- \\
\hline $02 / 26 / 92$ & 510,000 & 411 & 9.5 & .02 & 1.9 & .05 & .05 & 7.2 \\
\hline $02 / 26 / 92$ & 510,000 & 411 & 9.5 & .02 & 2.0 & .05 & .05 & -- \\
\hline $05 / 14 / 92$ & 611,000 & 410 & 18.5 & .01 & 3.0 & .03 & .07 & 6.6 \\
\hline $05 / 14 / 92$ & 611,000 & 410 & 18.5 & $<.01$ & 2.9 & .04 & .06 & -- \\
\hline $05 / 26 / 92$ & 466,000 & 404 & 24.0 & .01 & 1.9 & .02 & .06 & -- \\
\hline $05 / 26 / 92$ & 466,000 & 404 & 24.0 & $<.01$ & 2.8 & .04 & .06 & -- \\
\hline $06 / 17 / 92$ & 541,000 & 392 & 25.5 & $<.01$ & 1.4 & .03 & .07 & 4.3 \\
\hline $06 / 17 / 92$ & 541,000 & 392 & 25.5 & $<.01$ & 1.3 & .02 & .05 & -- \\
\hline $07 / 15 / 92$ & 449,000 & 396 & 28.0 & $<.01$ & 1.2 & .05 & .07 & -- \\
\hline 08/18/92 & 695,000 & 360 & 26.0 & $<.01$ & 1.8 & .04 & .09 & 8.0 \\
\hline $08 / 18 / 92$ & 695,000 & 360 & 26.0 & $<.01$ & 1.7 & .02 & .09 & -- \\
\hline $09 / 15 / 92$ & 339,000 & -- & 26.5 & $<.01$ & .88 & .04 & .08 & -- \\
\hline $10 / 14 / 92$ & 335,000 & 384 & 21.0 & $<.01$ & 1.3 & .03 & .09 & 7.8 \\
\hline $12 / 15 / 92$ & 808,000 & -- & 7.0 & .03 & 2.2 & .03 & .07 & 7.3 \\
\hline $01 / 26 / 93$ & $1,103,000$ & 334 & 5.5 & .04 & 1.7 & .06 & .06 & 7.2 \\
\hline 03/03/93 & 894,000 & 386 & 6.0 & .02 & 1.5 & .04 & .05 & 7.0 \\
\hline $04 / 20 / 93$ & $1,492,000$ & 319 & 9.5 & .03 & 1.6 & .02 & .05 & 6.5 \\
\hline $05 / 24 / 93$ & $1,516,000$ & 388 & 22.0 & $<.01$ & 1.6 & .04 & .07 & 7.0 \\
\hline $06 / 22 / 93$ & 917,000 & 426 & -- & $<.01$ & 2.2 & .03 & .07 & 7.2 \\
\hline $08 / 23 / 93$ & $1,130,000$ & 408 & 29.5 & $<.01$ & 1.7 & .03 & .10 & 10 \\
\hline $11 / 08 / 93$ & 486,000 & 469 & 12.5 & .03 & 1.8 & .03 & .08 & 7.7 \\
\hline $01 / 24 / 94$ & 730,000 & 346 & 3.0 & .01 & 1.1 & .06 & .04 & 6.9 \\
\hline
\end{tabular}


Table 8. Results of analysis of streamflow, physical properties, and nutrients for water samples collected from three sampling sites in the lower Mississippi River, Louisiana, 1991-2003.-Continued

[Streamflow data from U.S. Army Corps of Engineers, New Orleans District. Physical property data from various collectors. Nutrient data from U.S. Geological Survey National Water-Quality Laboratory, Lakewood, Colorado; $\mathrm{ft}^{3} / \mathrm{s}$, cubic feet per second; $\mu \mathrm{S} / \mathrm{cm}$, microsiemens per centimeter at 25 degrees Celsius; ${ }^{\circ} \mathrm{C}$, degrees Celsius; $\mathrm{mg} / \mathrm{L}$, milligrams per liter; $<$, less than; --, no data]

\begin{tabular}{|c|c|c|c|c|c|c|c|c|}
\hline $\begin{array}{c}\text { Collection } \\
\text { (month/day/ } \\
\text { year) }\end{array}$ & $\begin{array}{c}\text { Streamflow } \\
\left(\mathrm{ft}^{3} / \mathrm{s}\right)\end{array}$ & $\begin{array}{c}\text { Specific } \\
\text { conductance } \\
(\mu \mathrm{S} / \mathrm{cm})\end{array}$ & $\begin{array}{c}\text { Water } \\
\text { temperature } \\
\left({ }^{\circ} \mathrm{C}\right)\end{array}$ & $\begin{array}{c}\text { Nitrite as } \\
\text { nitrogen } \\
\text { (mg/L) }\end{array}$ & $\begin{array}{l}\text { Nitrite plus } \\
\text { nitrate as } \\
\text { nitrogen } \\
\text { (mg/L) }\end{array}$ & $\begin{array}{l}\text { Ammonia } \\
\text { as nitrogen } \\
(\mathrm{mg} / \mathrm{L})\end{array}$ & $\begin{array}{c}\text { Orthophosphate } \\
\text { as phosphorus } \\
\text { (mg/L) }\end{array}$ & $\begin{array}{c}\text { Silica as } \\
\mathrm{SiO}_{2}(\mathrm{mg} / \mathrm{L})\end{array}$ \\
\hline \multicolumn{9}{|c|}{ Mississippi River near St. Francisville, Louisiana, station 07373420 (fig. 1)—Continued } \\
\hline $03 / 14 / 94$ & $1,277,000$ & 305 & 8.0 & 0.02 & 1.3 & 0.06 & 0.04 & 6.0 \\
\hline $06 / 28 / 94$ & 492,000 & 412 & 29.0 & $<.01$ & .95 & .03 & .05 & 5.1 \\
\hline 08/30/94 & 392,000 & 436 & 29.0 & $<.01$ & .73 & .03 & .06 & 2.9 \\
\hline $10 / 20 / 94$ & 255,000 & 408 & 20.0 & $<.01$ & .79 & $<.02$ & .08 & 6.6 \\
\hline $01 / 23 / 95$ & 680,000 & 356 & 8.0 & .01 & 1.0 & .04 & -- & 6.2 \\
\hline $02 / 21 / 95$ & 482,000 & 350 & 5.0 & $<.01$ & 1.2 & .04 & .04 & 7.1 \\
\hline $04 / 24 / 95$ & 695,000 & 380 & 18.0 & .02 & 1.7 & $<.02$ & .04 & 6.1 \\
\hline $06 / 14 / 95$ & $1,620,000$ & 361 & 25.5 & $<.01$ & 1.4 & $<.01$ & .06 & 7.9 \\
\hline 08/07/95 & 477,000 & 477 & 28.0 & $<.01$ & 1.5 & .02 & .08 & 8.2 \\
\hline $10 / 12 / 95$ & 271,000 & 506 & 27.5 & $<.01$ & .50 & $<.02$ & .08 & 7.3 \\
\hline $10 / 17 / 95$ & 360,000 & 496 & 21.0 & .01 & .73 & $<.01$ & .08 & 7.4 \\
\hline $12 / 06 / 95$ & 395,000 & 484 & 9.5 & $<.01$ & 1.3 & .03 & .08 & 7.2 \\
\hline 02/07/96 & $1,040,000$ & 323 & 2.0 & .02 & 1.6 & .09 & .03 & 6.2 \\
\hline 03/27/96 & 757,000 & 328 & 9.0 & .04 & 1.2 & .04 & .08 & 6.8 \\
\hline $04 / 16 / 96$ & 835,000 & 339 & 12.0 & .02 & 1.4 & $<.02$ & .06 & 6.2 \\
\hline 05/01/96 & 899,000 & 408 & 17.4 & .01 & 1.4 & .02 & .06 & 5.8 \\
\hline 05/17/96 & $1,198,000$ & 327 & 19.8 & $<.01$ & 1.2 & $<.02$ & .06 & 6.2 \\
\hline $05 / 31 / 96$ & $1,390,000$ & 313 & 24.0 & $<.01$ & 1.1 & .03 & .08 & 6.7 \\
\hline $06 / 11 / 96$ & $1,409,000$ & 319 & 23.5 & $<.01$ & 1.5 & .02 & .07 & 6.5 \\
\hline $06 / 21 / 96$ & $1,327,000$ & 337 & 25.0 & $<.01$ & 2.1 & .03 & -- & 7.6 \\
\hline $06 / 25 / 96$ & $1,287,000$ & 337 & 34.0 & $<.01$ & 2.2 & .03 & -- & 6.7 \\
\hline 07/23/96 & 493,000 & 482 & 29.0 & $<.01$ & 2.4 & .03 & .11 & 7.6 \\
\hline 09/03/96 & 400,000 & 470 & 28.0 & $<.01$ & .79 & $<.02$ & .06 & 6.2 \\
\hline 09/17/96 & 323,000 & 471 & 27.5 & $<.01$ & .85 & $<.02$ & -- & 5.6 \\
\hline $11 / 25 / 96$ & 782,000 & 345 & 11.5 & .01 & .73 & $<.02$ & .05 & 6.0 \\
\hline $01 / 15 / 97$ & 798,000 & 328 & 9.5 & .03 & 1.3 & .03 & .04 & 5.9 \\
\hline $02 / 03 / 97$ & 846,000 & 323 & 6.0 & .03 & 1.4 & .04 & .03 & 6.9 \\
\hline $02 / 20 / 97$ & $1,022,000$ & 320 & 6.5 & .03 & 1.2 & .04 & .04 & 6.3 \\
\hline $03 / 14 / 97$ & $1,547,000$ & 275 & 14.0 & .03 & 1.1 & .03 & .04 & 5.7 \\
\hline $03 / 26 / 97$ & $1,899,000$ & 256 & 13.0 & .03 & 1.3 & $<.02$ & .04 & 6.1 \\
\hline 04/08/97 & $1,731,000$ & 299 & 15.0 & .02 & 1.3 & .03 & .05 & 5.7 \\
\hline $04 / 08 / 97$ & $1,731,000$ & 299 & 15.0 & .02 & 1.3 & .02 & .04 & 6.2 \\
\hline $04 / 24 / 97$ & $1,122,000$ & 345 & 15.0 & .01 & 1.4 & $<.02$ & .06 & 6.9 \\
\hline
\end{tabular}


Table 8. Results of analysis of streamflow, physical properties, and nutrients for water samples collected from three sampling sites in the lower Mississippi River, Louisiana, 1991-2003.-Continued

[Streamflow data from U.S. Army Corps of Engineers, New Orleans District. Physical property data from various collectors. Nutrient data from U.S. Geological Survey National Water-Quality Laboratory, Lakewood, Colorado; $\mathrm{ft}^{3} / \mathrm{s}$, cubic feet per second; $\mu \mathrm{S} / \mathrm{cm}$, microsiemens per centimeter at 25 degrees Celsius; ${ }^{\circ} \mathrm{C}$, degrees Celsius; $\mathrm{mg} / \mathrm{L}$, milligrams per liter; <, less than; --, no data]

\begin{tabular}{|c|c|c|c|c|c|c|c|c|}
\hline $\begin{array}{c}\text { Collection } \\
\text { (month/day/ } \\
\text { year) }\end{array}$ & $\begin{array}{l}\text { Streamflow } \\
\qquad\left(\mathrm{ft}^{3} / \mathrm{s}\right)\end{array}$ & $\begin{array}{c}\text { Specific } \\
\text { conductance } \\
(\mu \mathrm{S} / \mathrm{cm})\end{array}$ & $\begin{array}{c}\text { Water } \\
\text { temperature } \\
\left({ }^{\circ} \mathrm{C}\right)\end{array}$ & $\begin{array}{l}\text { Nitrite as } \\
\text { nitrogen } \\
\text { (mg/L) }\end{array}$ & $\begin{array}{c}\text { Nitrite plus } \\
\text { nitrate as } \\
\text { nitrogen } \\
\text { (mg/L) }\end{array}$ & $\begin{array}{l}\text { Ammonia } \\
\text { as nitrogen } \\
\text { (mg/L) }\end{array}$ & $\begin{array}{c}\text { Orthophosphate } \\
\text { as phosphorus } \\
\text { (mg/L) }\end{array}$ & $\begin{array}{c}\text { Silica as } \\
\mathrm{SiO}_{2}(\mathrm{mg} / \mathrm{L})\end{array}$ \\
\hline \multicolumn{9}{|c|}{ Mississippi River near St. Francisville, Louisiana, station 07373420 (fig. 1)—Continued } \\
\hline 05/06/97 & $1,006,000$ & 361 & 16.0 & 0.03 & 1.4 & $<0.02$ & 0.05 & 6.7 \\
\hline 06/09/97 & 910,000 & 408 & 21.6 & $<.01$ & 1.3 & $<.02$ & .05 & 4.1 \\
\hline 06/19/97 & $1,079,000$ & 353 & 23.0 & $<.01$ & 2.0 & .02 & .06 & 5.2 \\
\hline 07/09/97 & 942,000 & 379 & 28.5 & $<.01$ & 1.3 & $<.02$ & .08 & 5.2 \\
\hline 08/04/97 & 437,000 & 460 & 30.5 & $<.01$ & 1.2 & $<.02$ & .09 & 5.6 \\
\hline 08/26/97 & 402,000 & 473 & 28.0 & $<.01$ & .79 & $<.02$ & .06 & 2.6 \\
\hline 09/23/97 & 285,000 & 503 & 27.0 & $<.01$ & .66 & $<.02$ & .08 & 5.7 \\
\hline $11 / 17 / 97$ & 359,000 & 465 & 24.0 & $<.01$ & .90 & $<.02$ & -- & 8.2 \\
\hline $12 / 16 / 97$ & 416,000 & 540 & 7.0 & $<.01$ & 1.0 & $<.02$ & .06 & 5.8 \\
\hline $01 / 28 / 98$ & $1,166,000$ & 310 & 7.0 & .02 & 1.2 & .03 & .05 & 6.6 \\
\hline 02/27/98 & $1,059,000$ & 316 & 8.5 & .01 & 1.2 & .03 & .05 & 5.9 \\
\hline 03/18/98 & $1,002,000$ & 350 & 9.0 & .03 & 1.6 & .03 & .04 & 6.2 \\
\hline 04/30/98 & $1,266,000$ & 337 & 17.0 & .02 & 2.0 & .04 & .06 & 6.6 \\
\hline 05/13/98 & $1,506,000$ & 360 & 20.0 & .02 & 1.9 & .03 & .06 & 6.1 \\
\hline 05/28/98 & $1,231,000$ & 341 & 25.0 & .02 & 1.6 & $<.02$ & .06 & 5.7 \\
\hline 06/09/98 & 739,000 & 350 & 26.0 & .01 & 2.0 & .05 & .08 & 6.5 \\
\hline 06/25/98 & 958,000 & 370 & 26.0 & $<.01$ & 2.1 & .03 & -- & 7.0 \\
\hline 07/09/98 & $1,053,000$ & 354 & 26.0 & $<.01$ & 2.6 & .04 & .11 & 7.9 \\
\hline 07/30/98 & 639,000 & 375 & 30.0 & $<.01$ & 1.8 & .02 & -- & 8.8 \\
\hline 08/19/98 & 578,000 & 321 & 28.5 & .11 & .59 & .07 & .10 & 6.3 \\
\hline 09/28/98 & 309,000 & 471 & 27.5 & $<.01$ & .92 & $<.02$ & .08 & 5.3 \\
\hline $11 / 18 / 98$ & 592,000 & 419 & 13.5 & $<.01$ & 1.3 & .02 & .10 & 8.0 \\
\hline $12 / 08 / 98$ & 410,000 & 473 & 14.7 & .03 & 1.5 & .04 & .11 & 9.1 \\
\hline 01/12/99 & 503,000 & 375 & 4.5 & .01 & 1.1 & .08 & .05 & 7.0 \\
\hline 02/10/99 & $1,403,000$ & 311 & 10.5 & .01 & 1.6 & .03 & .05 & 6.2 \\
\hline $03 / 15 / 99$ & 982,000 & 387 & 9.0 & .02 & 1.6 & .03 & .06 & 6.4 \\
\hline 04/19/99 & 744,000 & 375 & 17.4 & .02 & 1.1 & $<.02$ & .05 & 5.1 \\
\hline 04/28/99 & 925,000 & 458 & 17.0 & $<.01$ & 2.0 & .03 & .07 & 6.7 \\
\hline 05/06/99 & $1,105,000$ & 446 & -- & $<.01$ & 2.8 & .04 & .07 & 7.9 \\
\hline 05/26/99 & $1,026,000$ & 395 & 23.0 & $<.01$ & 2.2 & $<.02$ & .07 & 7.2 \\
\hline 06/10/99 & 806,000 & 452 & 26.0 & $<.01$ & 2.9 & $<.02$ & .10 & 8.0 \\
\hline 06/24/99 & 685,000 & 444 & 26.5 & $<.01$ & 2.6 & .03 & .11 & 8.9 \\
\hline 07/21/99 & 677,000 & 396 & 29.4 & $<.01$ & 2.0 & $<.02$ & .10 & 7.7 \\
\hline
\end{tabular}


Table 8. Results of analysis of streamflow, physical properties, and nutrients for water samples collected from three sampling sites in the lower Mississippi River, Louisiana, 1991-2003.-Continued

[Streamflow data from U.S. Army Corps of Engineers, New Orleans District. Physical property data from various collectors. Nutrient data from U.S Geological Survey National Water-Quality Laboratory, Lakewood, Colorado; $\mathrm{ft}^{3} / \mathrm{s}$, cubic feet per second; $\mu \mathrm{S} / \mathrm{cm}$, microsiemens per centimeter at 25 degrees Celsius; ${ }^{\circ} \mathrm{C}$, degrees Celsius; $\mathrm{mg} / \mathrm{L}$, milligrams per liter; <, less than; --, no data]

\begin{tabular}{|c|c|c|c|c|c|c|c|c|}
\hline $\begin{array}{c}\text { Collection } \\
\text { (month/day/ } \\
\text { year) }\end{array}$ & $\begin{array}{c}\text { Streamflow } \\
\left(\mathrm{ft}^{3} / \mathrm{s}\right)\end{array}$ & $\begin{array}{c}\text { Specific } \\
\text { conductance } \\
(\mu \mathrm{S} / \mathrm{cm})\end{array}$ & $\begin{array}{c}\text { Water } \\
\text { temperature } \\
\left({ }^{\circ} \mathrm{C}\right)\end{array}$ & $\begin{array}{l}\text { Nitrite as } \\
\text { nitrogen } \\
\text { (mg/L) }\end{array}$ & $\begin{array}{c}\text { Nitrite plus } \\
\text { nitrate as } \\
\text { nitrogen } \\
\text { (mg/L) }\end{array}$ & $\begin{array}{l}\text { Ammonia } \\
\text { as nitrogen } \\
\text { (mg/L) }\end{array}$ & $\begin{array}{l}\text { Orthophosphate } \\
\text { as phosphorus } \\
\text { (mg/L) }\end{array}$ & $\begin{array}{c}\text { Silica as } \\
\mathrm{SiO}_{2}(\mathrm{mg} / \mathrm{L})\end{array}$ \\
\hline \multicolumn{9}{|c|}{ Mississippi River near St. Francisville, Louisiana, station 07373420 (fig. 1)—Continued } \\
\hline 08/05/99 & 448,000 & 461 & 32.3 & $<0.01$ & 1.8 & $<0.02$ & 0.10 & 7.4 \\
\hline 09/14/99 & 242,000 & 522 & 28.2 & $<.01$ & .95 & $<.02$ & .09 & 7.0 \\
\hline $11 / 11 / 99$ & 220,000 & -- & -- & $<.01$ & .72 & $<.02$ & .07 & 6.6 \\
\hline $12 / 01 / 99$ & 198,000 & -- & -- & $<.01$ & .74 & .04 & .05 & 6.7 \\
\hline 01/27/00 & 280,000 & 425 & 7.0 & .01 & 1.1 & .06 & .05 & 5.2 \\
\hline $02 / 17 / 00$ & 211,000 & 486 & 8.8 & .01 & 1.1 & .08 & .04 & 7.2 \\
\hline $03 / 14 / 00$ & 653,000 & 401 & 13 & .03 & 1.4 & $<.02$ & .05 & 6.2 \\
\hline $03 / 29 / 00$ & 697,000 & 413 & 14.2 & .03 & 1.5 & $<.02$ & .06 & 6.2 \\
\hline $04 / 12 / 00$ & 694,000 & 416 & 16.1 & .03 & 1.1 & .02 & .05 & 5.7 \\
\hline 05/10/00 & 625,000 & 403 & 20.8 & -- & 1.2 & $<.02$ & .06 & 4.8 \\
\hline $05 / 23 / 00$ & 399,000 & 374 & 23.6 & $<.01$ & 1.2 & $<.02$ & .07 & 5.0 \\
\hline 06/06/00 & 610,000 & 539 & 25.4 & $<.01$ & 1.1 & $<.02$ & .05 & 3.8 \\
\hline $07 / 18 / 00$ & 591,000 & 440 & 30.5 & $<.01$ & 2.8 & $<.02$ & .11 & 7.9 \\
\hline $08 / 25 / 00$ & 321,000 & 429 & 30.5 & $<.01$ & .98 & $<.02$ & .08 & 4.8 \\
\hline 09/21/00 & 224,000 & 469 & 26.2 & $<.01$ & .64 & $<.02$ & .07 & 3.3 \\
\hline $11 / 28 / 00$ & 375,000 & 453 & 10.6 & $<.01$ & 1.0 & .03 & .09 & 6.6 \\
\hline $12 / 14 / 00$ & 294,000 & 467 & 7.1 & $<.01$ & 1.2 & $<.02$ & .07 & 6.8 \\
\hline $01 / 24 / 01$ & 341,000 & 375 & 4.5 & $<.01$ & 1.2 & .15 & .03 & 6.2 \\
\hline 02/27/01 & $1,084,000$ & 320 & 7.9 & .02 & 1.7 & .07 & .05 & 4.7 \\
\hline $03 / 14 / 01$ & $1,246,000$ & 314 & 9.5 & .03 & 1.6 & .03 & .05 & 5.9 \\
\hline $04 / 02 / 01$ & 958,000 & 347 & 10.3 & .01 & .97 & $<.02$ & .03 & 5.9 \\
\hline 04/16/01 & 706,000 & 403 & 17.1 & .04 & 2.5 & $<.02$ & .08 & -- \\
\hline 04/23/01 & 820,000 & 380 & 17.7 & .01 & 2.4 & $<.02$ & .09 & 6.8 \\
\hline 05/07/01 & 684,000 & 403 & 20.5 & .01 & 2.8 & $<.02$ & .08 & 6.6 \\
\hline $05 / 21 / 01$ & 576,000 & 405 & 23.8 & $<.01$ & 3.0 & $<.02$ & .10 & 9.2 \\
\hline $06 / 25 / 01$ & 869,000 & 385 & 26.9 & $<.01$ & 2.7 & .04 & .09 & 7.0 \\
\hline 07/10/01 & 557,000 & 414 & 29.0 & $<.01$ & 2.7 & $<.02$ & .11 & 7.9 \\
\hline 07/18/01 & 532,000 & 434 & 29.4 & $<.01$ & 2.4 & $<.02$ & .11 & 8.3 \\
\hline 08/27/01 & 315,000 & 398 & 30.0 & .01 & .90 & $<.02$ & .08 & 3.8 \\
\hline 09/19/01 & 278,000 & 356 & 27.5 & .01 & .51 & $<.02$ & .09 & 4.5 \\
\hline $10 / 30 / 01$ & 445,000 & 383 & 17.7 & $<.01$ & .86 & $<.02$ & .09 & 5.2 \\
\hline $12 / 18 / 01$ & 836,000 & 272 & 12.3 & .02 & .87 & $<.02$ & .08 & 5.0 \\
\hline $01 / 22 / 02$ & 382,000 & 346 & 6.9 & .01 & 1.2 & .05 & .05 & 7.4 \\
\hline
\end{tabular}


Table 8. Results of analysis of streamflow, physical properties, and nutrients for water samples collected from three sampling sites in the lower Mississippi River, Louisiana, 1991-2003.-Continued

[Streamflow data from U.S. Army Corps of Engineers, New Orleans District. Physical property data from various collectors. Nutrient data from U.S. Geological Survey National Water-Quality Laboratory, Lakewood, Colorado; $\mathrm{ft}^{3} / \mathrm{s}$, cubic feet per second; $\mu \mathrm{S} / \mathrm{cm}$, microsiemens per centimeter at 25 degrees Celsius; ${ }^{\circ} \mathrm{C}$, degrees Celsius; $\mathrm{mg} / \mathrm{L}$, milligrams per liter; <, less than; --, no data]

\begin{tabular}{|c|c|c|c|c|c|c|c|c|}
\hline $\begin{array}{c}\text { Collection } \\
\text { (month/day/ } \\
\text { year) }\end{array}$ & $\begin{array}{c}\text { Streamflow } \\
\left(\mathrm{ft}^{3} / \mathrm{s}\right)\end{array}$ & $\begin{array}{c}\text { Specific } \\
\text { conductance } \\
(\mu \mathrm{S} / \mathrm{cm})\end{array}$ & $\begin{array}{c}\text { Water } \\
\text { temperature } \\
\left({ }^{\circ} \mathrm{C}\right)\end{array}$ & $\begin{array}{l}\text { Nitrite as } \\
\text { nitrogen } \\
\text { (mg/L) }\end{array}$ & $\begin{array}{l}\text { Nitrite plus } \\
\text { nitrate as } \\
\text { nitrogen } \\
\text { (mg/L) }\end{array}$ & $\begin{array}{l}\text { Ammonia } \\
\text { as nitrogen } \\
(\mathrm{mg} / \mathrm{L})\end{array}$ & $\begin{array}{l}\text { Orthophosphate } \\
\text { as phosphorus } \\
\text { (mg/L) }\end{array}$ & $\begin{array}{c}\text { Silica as } \\
\mathrm{SiO}_{2}(\mathrm{mg} / \mathrm{L})\end{array}$ \\
\hline \multicolumn{9}{|c|}{ Mississippi River near St. Francisville, Louisiana, station 07373420 (fig .1)—Continued } \\
\hline 02/04/02 & 946,000 & 310 & 10.0 & 0.01 & 1.1 & 0.03 & 0.05 & 4.8 \\
\hline 02/18/02 & 855,000 & 266 & 10.3 & .02 & 1.3 & .03 & .05 & 5.4 \\
\hline $03 / 13 / 02$ & 516,000 & 387 & 8.6 & $<.01$ & 1.9 & .03 & .05 & 5.5 \\
\hline $03 / 25 / 02$ & 919,000 & 311 & 11.5 & .02 & 1.5 & .02 & .05 & 4.0 \\
\hline $04 / 10 / 02$ & $1,375,000$ & 264 & 13.5 & .02 & 1.0 & $<.02$ & .04 & 5.2 \\
\hline $04 / 22 / 02$ & $1,055,000$ & 296 & 19.1 & .03 & 1.3 & $<.02$ & .05 & 4.5 \\
\hline 05/07/02 & $1,019,000$ & 331 & 19.8 & $<.01$ & 1.5 & $<.02$ & .06 & 5.2 \\
\hline $05 / 28 / 02$ & $1,409,000$ & 288 & 20.7 & .01 & 1.3 & $<.02$ & .05 & 6.0 \\
\hline 08/05/02 & 364,000 & 430 & 30.3 & $<.01$ & 1.2 & $<.02$ & .07 & 4.4 \\
\hline $08 / 19 / 02$ & 295,000 & 443 & 29.3 & $<.01$ & .68 & $<.02$ & .07 & 3.8 \\
\hline $09 / 09 / 02$ & 336,000 & 414 & 28.6 & $<.01$ & .94 & $<.02$ & .10 & 4.3 \\
\hline $11 / 12 / 02$ & 446,000 & 362 & 15.0 & .01 & 1.1 & $<.02$ & .08 & 7.0 \\
\hline $01 / 14 / 03$ & 747,000 & 285 & 6.3 & .01 & 1.2 & $<.02$ & .05 & 4.9 \\
\hline $01 / 28 / 03$ & 370,000 & 345 & 5.5 & .01 & 1.2 & .03 & .04 & 6.4 \\
\hline $02 / 12 / 03$ & 333,000 & 360 & 6.0 & .01 & 1.1 & .03 & .03 & 6.4 \\
\hline $02 / 24 / 03$ & 871,000 & 302 & 7.4 & $<.01$ & .85 & .04 & .03 & 4.5 \\
\hline 03/10/03 & $1,279,000$ & 255 & 7.3 & .01 & .90 & .03 & .03 & 5.0 \\
\hline $03 / 24 / 03$ & 844,000 & 289 & 11.6 & .01 & 1.1 & .02 & .03 & 4.9 \\
\hline $04 / 15 / 03$ & 550,000 & 366 & 15.5 & .04 & 1.3 & $<.02$ & .04 & 4.5 \\
\hline $04 / 29 / 03$ & 650,000 & 364 & 18.4 & $<.01$ & 1.0 & $<.02$ & .04 & 3.7 \\
\hline $05 / 12 / 03$ & 778,000 & 362 & 22.3 & $<.01$ & 1.1 & $<.02$ & .06 & 4.1 \\
\hline $05 / 27 / 03$ & $1,381,000$ & 303 & 22.0 & $<.01$ & 1.4 & $<.02$ & .06 & 5.3 \\
\hline $06 / 16 / 03$ & 771,000 & 354 & 23.5 & $<.01$ & 1.4 & $<.02$ & .07 & 5.9 \\
\hline $07 / 14 / 03$ & 462,000 & 371 & 28.7 & $<.01$ & 1.3 & $<.02$ & .07 & 5.1 \\
\hline $08 / 12 / 03$ & 516,000 & 397 & 28.9 & $<.01$ & 1.6 & $<.02$ & .09 & 4.4 \\
\hline $09 / 23 / 03$ & 411,000 & 351 & 25.4 & $<.01$ & .77 & $<.02$ & .07 & 6.9 \\
\hline $10 / 27 / 03$ & 308,000 & 402 & 19.8 & .04 & .68 & $<.02$ & .06 & 5.3 \\
\hline $12 / 09 / 03$ & 815,000 & 298 & 9.6 & .01 & 1.1 & $<.02$ & .05 & 7.3 \\
\hline \multicolumn{9}{|c|}{ Mississippi River at Baton Rouge, Louisiana, station 07374000 (fig. 1) } \\
\hline $04 / 11 / 91$ & $1,153,000$ & 366 & 16.5 & .01 & 2.0 & .02 & .03 & -- \\
\hline $04 / 17 / 91$ & $1,175,000$ & 306 & 18.0 & .02 & 1.9 & .02 & .04 & -- \\
\hline $04 / 24 / 91$ & $1,198,000$ & 293 & 18.0 & .01 & 1.7 & .02 & .05 & -- \\
\hline 05/01/91 & $1,351,000$ & 300 & 18.5 & $<.01$ & 1.5 & .02 & .04 & -- \\
\hline 05/06/91 & $1,380,000$ & 280 & 19.0 & .01 & 1.6 & .02 & .04 & -- \\
\hline
\end{tabular}


Table 8. Results of analysis of streamflow, physical properties, and nutrients for water samples collected from three sampling sites in the lower Mississippi River, Louisiana, 1991-2003.-Continued

[Streamflow data from U.S. Army Corps of Engineers, New Orleans District. Physical property data from various collectors. Nutrient data from U.S Geological Survey National Water-Quality Laboratory, Lakewood, Colorado; $\mathrm{ft}^{3} / \mathrm{s}$, cubic feet per second; $\mu \mathrm{S} / \mathrm{cm}$, microsiemens per centimeter at 25 degrees Celsius; ${ }^{\circ} \mathrm{C}$, degrees Celsius; $\mathrm{mg} / \mathrm{L}$, milligrams per liter; <, less than; --, no data]

\begin{tabular}{|c|c|c|c|c|c|c|c|c|}
\hline $\begin{array}{l}\text { Collection } \\
\text { (month/day/ } \\
\text { year) }\end{array}$ & $\begin{array}{c}\text { Streamflow } \\
\left(\mathrm{ft}^{3} / \mathrm{s}\right)\end{array}$ & $\begin{array}{c}\text { Specific } \\
\text { conductance } \\
(\mu \mathrm{S} / \mathrm{cm})\end{array}$ & $\begin{array}{c}\text { Water } \\
\text { temperature } \\
\left({ }^{\circ} \mathrm{C}\right)\end{array}$ & $\begin{array}{l}\text { Nitrite as } \\
\text { nitrogen } \\
\text { (mg/L) }\end{array}$ & $\begin{array}{l}\text { Nitrite plus } \\
\text { nitrate as } \\
\text { nitrogen } \\
\text { (mg/L) }\end{array}$ & $\begin{array}{l}\text { Ammonia } \\
\text { as nitrogen } \\
(\mathrm{mg} / \mathrm{L})\end{array}$ & $\begin{array}{l}\text { Orthophosphate } \\
\text { as phosphorus } \\
\text { (mg/L) }\end{array}$ & $\begin{array}{c}\text { Silica as } \\
\mathrm{SiO}_{2} \text { (mg/L) }\end{array}$ \\
\hline \multicolumn{9}{|c|}{ Mississippi River at Baton Rouge, Louisiana, station 07374000 (fig. 1)_-Continued } \\
\hline 05/09/91 & $1,319,000$ & 277 & 19.0 & $<0.01$ & 1.7 & 0.02 & 0.05 & -- \\
\hline 05/13/91 & $1,275,000$ & 281 & 21.0 & $<.01$ & 1.8 & .02 & .05 & -- \\
\hline 05/16/91 & $1,224,000$ & 295 & 22.0 & .01 & 1.9 & .04 & .04 & -- \\
\hline 05/20/91 & $1,159,000$ & 291 & 22.5 & .01 & 1.9 & .04 & .04 & -- \\
\hline $05 / 24 / 91$ & $1,127,000$ & 294 & 23.0 & .01 & 1.9 & .07 & .07 & -- \\
\hline 05/28/91 & $1,070,000$ & 311 & 24.5 & $<.01$ & 2.0 & .05 & .06 & -- \\
\hline 05/30/91 & $1,050,000$ & 316 & 25.0 & $<.01$ & 1.9 & .03 & .07 & -- \\
\hline 06/03/91 & 995,000 & 336 & 26.0 & $<.01$ & 2.0 & .02 & .06 & -- \\
\hline 06/06/91 & $1,003,000$ & 353 & 27.0 & $<.01$ & 2.4 & .02 & .07 & -- \\
\hline $06 / 10 / 91$ & $1,015,000$ & 307 & 26.5 & $<.01$ & 2.0 & .03 & .07 & -- \\
\hline 06/13/91 & 952,000 & 338 & 27.0 & $<.01$ & 2.3 & .02 & .07 & -- \\
\hline 06/17/91 & 826,000 & 382 & 27.5 & .01 & 2.6 & .04 & .09 & -- \\
\hline 06/20/91 & 753,000 & 403 & 28.0 & $<.01$ & 2.6 & .05 & .07 & -- \\
\hline $06 / 24 / 91$ & 664,000 & 410 & 28.0 & $<.01$ & 3.3 & .04 & .09 & -- \\
\hline $06 / 27 / 91$ & 649,000 & 409 & 28.0 & $<.01$ & $<.05$ & $<.01$ & .01 & -- \\
\hline 07/03/91 & 572,000 & 426 & 29.5 & $<.01$ & 2.9 & .02 & .10 & -- \\
\hline 07/08/91 & 507,000 & 413 & 29.0 & $<.01$ & 2.6 & .01 & .08 & -- \\
\hline 07/11/91 & 471,000 & 390 & 30.0 & $<.01$ & 2.6 & .03 & .10 & -- \\
\hline $07 / 17 / 91$ & 434,000 & 426 & 30.5 & $<.01$ & 2.7 & .04 & .10 & -- \\
\hline $07 / 23 / 91$ & 457,000 & 440 & 30.5 & $<.01$ & 2.1 & .02 & .08 & -- \\
\hline 07/23/91 & 457,000 & -- & -- & $<.01$ & 2.1 & .02 & .07 & -- \\
\hline $07 / 23 / 91$ & 457,000 & -- & -- & $<.01$ & 2.0 & .01 & .07 & -- \\
\hline 07/23/91 & 457,000 & -- & -- & $<.01$ & 2.0 & .01 & .07 & -- \\
\hline $07 / 23 / 91$ & 457,000 & -- & -- & $<.01$ & 2.0 & $<.01$ & .07 & -- \\
\hline 07/23/91 & 457,000 & -- & -- & $<.01$ & 2.0 & $<.01$ & .07 & -- \\
\hline 07/30/91 & 369,000 & 413 & 30.0 & $<.01$ & 1.8 & .04 & .08 & -- \\
\hline 08/06/91 & 330,000 & 450 & 30.5 & $<.01$ & 1.7 & .03 & .06 & -- \\
\hline $08 / 12 / 91$ & 302,000 & 456 & 30.5 & $<.01$ & 1.3 & .03 & .05 & -- \\
\hline 08/20/91 & 296,000 & 464 & 29.5 & .01 & .89 & .01 & .05 & -- \\
\hline $08 / 26 / 91$ & 288,000 & 451 & 29.5 & $<.01$ & .75 & .02 & .03 & -- \\
\hline 09/03/91 & 288,000 & 468 & 29.0 & $<.01$ & .74 & $<.01$ & .04 & -- \\
\hline 09/09/91 & 272,000 & 466 & 29.0 & $<.01$ & .82 & .03 & .04 & -- \\
\hline 09/18/91 & 248,000 & 425 & 30.5 & $<.01$ & .75 & .08 & .07 & -- \\
\hline
\end{tabular}


Table 8. Results of analysis of streamflow, physical properties, and nutrients for water samples collected from three sampling sites in the lower Mississippi River, Louisiana, 1991-2003.-Continued

[Streamflow data from U.S. Army Corps of Engineers, New Orleans District. Physical property data from various collectors. Nutrient data from U.S. Geological Survey National Water-Quality Laboratory, Lakewood, Colorado; $\mathrm{ft}^{3} / \mathrm{s}$, cubic feet per second; $\mu \mathrm{S} / \mathrm{cm}$, microsiemens per centimeter at 25 degrees Celsius; ${ }^{\circ} \mathrm{C}$, degrees Celsius; $\mathrm{mg} / \mathrm{L}$, milligrams per liter; <, less than; --, no data]

\begin{tabular}{|c|c|c|c|c|c|c|c|c|}
\hline $\begin{array}{c}\text { Collection } \\
\text { (month/day/ } \\
\text { year) }\end{array}$ & $\begin{array}{c}\text { Streamflow } \\
\left(\mathrm{ft}^{3} / \mathrm{s}\right)\end{array}$ & $\begin{array}{c}\text { Specific } \\
\text { conductance } \\
(\mu \mathrm{S} / \mathrm{cm})\end{array}$ & $\begin{array}{c}\text { Water } \\
\text { temperature } \\
\left({ }^{\circ} \mathrm{C}\right)\end{array}$ & $\begin{array}{c}\text { Nitrite as } \\
\text { nitrogen } \\
\text { (mg/L) }\end{array}$ & $\begin{array}{l}\text { Nitrite plus } \\
\text { nitrate as } \\
\text { nitrogen } \\
\text { (mg/L) }\end{array}$ & $\begin{array}{l}\text { Ammonia } \\
\text { as nitrogen } \\
(\mathrm{mg} / \mathrm{L})\end{array}$ & $\begin{array}{c}\text { Orthophosphate } \\
\text { as phosphorus } \\
\text { (mg/L) }\end{array}$ & $\begin{array}{c}\text { Silica as } \\
\mathrm{SiO}_{2} \text { (mg/L) }\end{array}$ \\
\hline \multicolumn{9}{|c|}{ Mississippi River at Baton Rouge, Louisiana, station 07374000 (fig. 1)—Continued } \\
\hline $09 / 23 / 91$ & 258,000 & 457 & 28.0 & 0.01 & 0.62 & 0.04 & 0.07 & -- \\
\hline $10 / 07 / 91$ & 261,000 & 447 & 23.0 & $<.01$ & .64 & .02 & .07 & -- \\
\hline $10 / 16 / 91$ & 264,000 & 479 & 22.0 & $<.01$ & .63 & .05 & .05 & -- \\
\hline $10 / 23 / 91$ & 232,000 & 489 & 20.5 & $<.01$ & .78 & .06 & .09 & -- \\
\hline $10 / 31 / 91$ & 256,000 & 453 & 21.0 & .02 & .83 & .05 & .03 & -- \\
\hline $11 / 07 / 91$ & 407,000 & 596 & 17.0 & $<.01$ & .63 & $<.01$ & .06 & -- \\
\hline $11 / 14 / 91$ & 405,000 & 462 & 12.0 & .03 & .77 & .05 & .07 & -- \\
\hline $11 / 19 / 91$ & 383,000 & 455 & 16.0 & $<.01$ & $<.05$ & $<.01$ & $<.01$ & -- \\
\hline $11 / 25 / 91$ & 483,000 & 407 & 13.0 & .02 & 1.0 & .07 & .06 & -- \\
\hline $12 / 02 / 91$ & 530,000 & 353 & 14.5 & .04 & 1.3 & .05 & .06 & -- \\
\hline $12 / 10 / 91$ & 794,000 & 312 & 10.0 & .03 & 1.2 & .07 & .06 & -- \\
\hline $12 / 17 / 91$ & $1,019,000$ & 318 & 10.5 & .03 & 1.1 & .03 & .05 & -- \\
\hline $12 / 24 / 91$ & $1,024,000$ & 280 & 10.0 & .03 & 1.1 & .05 & .06 & -- \\
\hline $12 / 31 / 91$ & 864,000 & 316 & 9.0 & .03 & 1.4 & .04 & .06 & -- \\
\hline $01 / 07 / 92$ & 756,000 & 341 & 8.5 & .02 & 1.8 & .05 & .06 & -- \\
\hline $01 / 29 / 92$ & 551,000 & -- & 6.5 & .03 & 1.6 & .05 & .06 & -- \\
\hline $02 / 06 / 92$ & 559,000 & 379 & 8.5 & .02 & 1.6 & .06 & .05 & -- \\
\hline $02 / 13 / 92$ & 416,000 & 416 & 11.0 & .01 & 1.9 & .04 & .05 & -- \\
\hline $02 / 20 / 92$ & 419,000 & 407 & 12.0 & .02 & 1.8 & .06 & .06 & -- \\
\hline $02 / 26 / 92$ & 510,000 & 434 & 10.5 & .02 & 2.1 & .07 & .06 & -- \\
\hline $03 / 05 / 92$ & 646,000 & 420 & 11.0 & .02 & 1.7 & .10 & .05 & -- \\
\hline $03 / 11 / 92$ & 706,000 & 378 & 12.5 & .03 & 2.0 & .04 & .05 & -- \\
\hline $03 / 19 / 92$ & 803,000 & 378 & 12.5 & .03 & 2.0 & .06 & .06 & -- \\
\hline $03 / 26 / 92$ & 924,000 & 328 & 12.5 & .01 & 1.6 & .03 & .06 & -- \\
\hline $03 / 30 / 92$ & 959,000 & 335 & 12.5 & .02 & 1.9 & .03 & .05 & -- \\
\hline $04 / 10 / 92$ & 805,000 & 370 & 13.0 & .02 & 2.3 & .03 & .06 & -- \\
\hline $04 / 15 / 92$ & 660,000 & 384 & 15.0 & .03 & 2.1 & .04 & .06 & -- \\
\hline $04 / 23 / 92$ & 493,000 & 430 & 18.5 & .02 & 2.3 & .04 & .06 & -- \\
\hline $04 / 30 / 92$ & 737,000 & 462 & 19.0 & $<.01$ & 1.2 & .03 & .04 & -- \\
\hline $05 / 06 / 92$ & 850,000 & 396 & 18.0 & $<.01$ & 2.1 & .02 & .06 & -- \\
\hline $05 / 14 / 92$ & 611,000 & 434 & 23.0 & $<.01$ & 2.9 & .03 & .07 & -- \\
\hline $05 / 20 / 92$ & 533,000 & 469 & 23.0 & .01 & 1.8 & .03 & .05 & -- \\
\hline $05 / 28 / 92$ & 443,000 & 456 & 25.0 & .02 & 1.8 & .04 & .06 & -- \\
\hline
\end{tabular}


Table 8. Results of analysis of streamflow, physical properties, and nutrients for water samples collected from three sampling sites in the lower Mississippi River, Louisiana, 1991-2003.-Continued

[Streamflow data from U.S. Army Corps of Engineers, New Orleans District. Physical property data from various collectors. Nutrient data from U.S Geological Survey National Water-Quality Laboratory, Lakewood, Colorado; $\mathrm{ft}^{3} / \mathrm{s}$, cubic feet per second; $\mu \mathrm{S} / \mathrm{cm}$, microsiemens per centimeter at 25 degrees Celsius; ${ }^{\circ} \mathrm{C}$, degrees Celsius; $\mathrm{mg} / \mathrm{L}$, milligrams per liter; <, less than; --, no data]

\begin{tabular}{|c|c|c|c|c|c|c|c|c|}
\hline $\begin{array}{l}\text { Collection } \\
\text { (month/day/ } \\
\text { year) }\end{array}$ & $\begin{array}{c}\text { Streamflow } \\
\left(\mathrm{ft}^{3} / \mathrm{s}\right)\end{array}$ & $\begin{array}{c}\text { Specific } \\
\text { conductance } \\
(\mu \mathrm{S} / \mathrm{cm})\end{array}$ & $\begin{array}{c}\text { Water } \\
\text { temperature } \\
\left({ }^{\circ} \mathrm{C}\right)\end{array}$ & $\begin{array}{c}\text { Nitrite as } \\
\text { nitrogen } \\
\text { (mg/L) }\end{array}$ & $\begin{array}{l}\text { Nitrite plus } \\
\text { nitrate as } \\
\text { nitrogen } \\
\text { (mg/L) }\end{array}$ & $\begin{array}{l}\text { Ammonia } \\
\text { as nitrogen } \\
\text { (mg/L) }\end{array}$ & $\begin{array}{c}\text { Orthophosphate } \\
\text { as phosphorus } \\
\text { (mg/L) }\end{array}$ & $\begin{array}{c}\text { Silica as } \\
\mathrm{SiO}_{2} \text { (mg/L) }\end{array}$ \\
\hline
\end{tabular}

\begin{tabular}{|c|c|c|c|c|c|c|c|c|}
\hline \multicolumn{9}{|c|}{ Mississippi River at Baton Rouge, Louisiana, station 07374000 (fig. 1)—Continued } \\
\hline 06/03/92 & 405,000 & 470 & 24.0 & $<0.01$ & 1.6 & 0.02 & 0.06 & -- \\
\hline $06 / 12 / 92$ & 500,000 & 443 & 25.0 & $<.01$ & 1.6 & .02 & .06 & -- \\
\hline 06/19/92 & 561,000 & 418 & 27.0 & $<.01$ & 1.3 & .03 & .06 & -- \\
\hline $06 / 25 / 92$ & 501,000 & -- & 29.0 & $<.01$ & 1.0 & .03 & .06 & -- \\
\hline 07/02/92 & 496,000 & -- & 27.0 & $<.01$ & 1.1 & .03 & .06 & -- \\
\hline 07/09/92 & 417,000 & -- & 28.0 & .01 & 1.1 & .04 & .07 & -- \\
\hline 07/16/92 & 449,000 & 427 & 27.0 & .01 & 1.4 & .06 & .08 & -- \\
\hline 07/23/92 & 500,000 & 438 & 28.5 & .01 & .97 & .06 & .07 & -- \\
\hline 07/31/92 & 628,000 & 415 & 28.5 & $<.01$ & 1.9 & .01 & .09 & -- \\
\hline 08/05/92 & 678,000 & 395 & 28.5 & $<.01$ & 2.1 & $<.01$ & .09 & -- \\
\hline 08/13/92 & 783,000 & 420 & 29.0 & $<.01$ & 2.0 & .02 & .09 & -- \\
\hline 08/21/92 & 609,000 & 388 & 27.0 & $<.01$ & 1.8 & .02 & .09 & -- \\
\hline 09/04/92 & 359,000 & -- & -- & $<.01$ & 1.4 & .04 & .09 & -- \\
\hline 09/09/92 & 396,000 & -- & -- & $<.01$ & 1.1 & .06 & .10 & -- \\
\hline 09/16/92 & 347,000 & 442 & 28.0 & $<.01$ & .84 & .04 & .07 & -- \\
\hline 09/23/92 & 400,000 & 463 & 26.0 & $<.01$ & .83 & .04 & .08 & -- \\
\hline 07/07/93 & 843,000 & 351 & 28.5 & $<.01$ & 2.5 & .02 & .10 & -- \\
\hline 07/09/93 & 835,000 & 345 & 28.5 & $<.01$ & 2.4 & .03 & .10 & -- \\
\hline 07/12/93 & 848,000 & 418 & 28.5 & $<.01$ & 2.5 & .03 & -- & -- \\
\hline $07 / 15 / 93$ & 910,000 & 414 & 28.5 & $<.01$ & 2.4 & .04 & -- & -- \\
\hline $07 / 19 / 93$ & 959,000 & -- & -- & $<.01$ & 2.2 & .05 & .08 & -- \\
\hline $07 / 22 / 93$ & 993,000 & 347 & 28.5 & $<.01$ & 2.0 & .05 & .07 & -- \\
\hline 07/26/93 & $1,028,000$ & 344 & 30.0 & $<.01$ & 2.1 & .01 & .07 & -- \\
\hline 07/29/93 & $1,054,000$ & 345 & 29.5 & $<.01$ & 2.0 & .03 & .08 & -- \\
\hline 08/02/93 & $1,088,000$ & 360 & 30.0 & $<.01$ & 2.0 & .03 & .08 & -- \\
\hline 08/05/93 & $1,110,000$ & 380 & 30.0 & $<.01$ & 2.1 & .03 & .09 & -- \\
\hline 08/06/93 & $1,115,000$ & 394 & 29.0 & $<.01$ & 2.1 & .03 & .09 & -- \\
\hline 08/09/93 & $1,137,000$ & 378 & 28.0 & $<.01$ & 1.9 & .03 & .09 & -- \\
\hline $08 / 12 / 93$ & $1,159,000$ & 358 & 28.5 & $<.01$ & 1.7 & .03 & .08 & -- \\
\hline 08/16/93 & $1,172,000$ & 364 & 28.5 & $<.01$ & 1.7 & .03 & .07 & -- \\
\hline 08/19/93 & $1,156,000$ & 381 & 29.5 & $<.01$ & 1.7 & .03 & .07 & -- \\
\hline $08 / 23 / 93$ & $1,130,000$ & 406 & 30.5 & $<.01$ & 1.7 & .02 & .09 & -- \\
\hline 08/26/93 & $1,106,000$ & 395 & 30.0 & $<.01$ & 1.5 & .03 & .09 & -- \\
\hline
\end{tabular}


Table 8. Results of analysis of streamflow, physical properties, and nutrients for water samples collected from three sampling sites in the lower Mississippi River, Louisiana, 1991-2003.-Continued

[Streamflow data from U.S. Army Corps of Engineers, New Orleans District. Physical property data from various collectors. Nutrient data from U.S. Geological Survey National Water-Quality Laboratory, Lakewood, Colorado; $\mathrm{ft}^{3} / \mathrm{s}$, cubic feet per second; $\mu \mathrm{S} / \mathrm{cm}$, microsiemens per centimeter at 25 degrees Celsius; ${ }^{\circ} \mathrm{C}$, degrees Celsius; $\mathrm{mg} / \mathrm{L}$, milligrams per liter; <, less than; --, no data]

\begin{tabular}{|c|c|c|c|c|c|c|c|c|}
\hline $\begin{array}{c}\text { Collection } \\
\text { (month/day/ } \\
\text { year) }\end{array}$ & $\begin{array}{c}\text { Streamflow } \\
\left(\mathrm{ft}^{3} / \mathrm{s}\right)\end{array}$ & $\begin{array}{c}\text { Specific } \\
\text { conductance } \\
(\mu \mathrm{S} / \mathrm{cm})\end{array}$ & $\begin{array}{c}\text { Water } \\
\text { temperature } \\
\left({ }^{\circ} \mathrm{C}\right)\end{array}$ & $\begin{array}{l}\text { Nitrite as } \\
\text { nitrogen } \\
\text { (mg/L) }\end{array}$ & $\begin{array}{l}\text { Nitrite plus } \\
\text { nitrate as } \\
\text { nitrogen } \\
\text { (mg/L) }\end{array}$ & $\begin{array}{c}\text { Ammonia } \\
\text { as nitrogen } \\
(\mathrm{mg} / \mathrm{L})\end{array}$ & $\begin{array}{l}\text { Orthophosphate } \\
\text { as phosphorus } \\
\text { (mg/L) }\end{array}$ & $\begin{array}{c}\text { Silica as } \\
\mathrm{SiO}_{2}(\mathrm{mg} / \mathrm{L})\end{array}$ \\
\hline \multicolumn{9}{|c|}{ Mississippi River at Baton Rouge, Louisiana, station 07374000 (fig. 1)_-Continued } \\
\hline 08/30/93 & $1,063,000$ & 389 & 30.0 & $<0.01$ & 1.4 & 0.03 & 0.09 & -- \\
\hline 09/02/93 & 998,000 & 394 & 30.0 & $<.01$ & 1.4 & .03 & .10 & -- \\
\hline 09/07/93 & 818,000 & 405 & 29.5 & $<.01$ & 1.4 & .02 & .09 & -- \\
\hline $09 / 16 / 93$ & 688,000 & 443 & 26.5 & $<.01$ & 1.8 & .02 & .11 & -- \\
\hline $09 / 23 / 93$ & 678,000 & 438 & 26.5 & $<.01$ & 1.7 & .03 & .11 & -- \\
\hline $10 / 01 / 93$ & 786,000 & 416 & 23.0 & $<.01$ & 1.5 & .03 & .10 & -- \\
\hline $10 / 08 / 93$ & 970,000 & 338 & 21.0 & $<.01$ & 1.1 & .04 & .08 & -- \\
\hline $10 / 15 / 93$ & 974,000 & 348 & 20.5 & $<.01$ & 1.3 & .04 & .08 & -- \\
\hline $10 / 22 / 93$ & 661,000 & 420 & 19.0 & $<.01$ & 1.5 & .03 & .09 & -- \\
\hline $11 / 05 / 93$ & 529,000 & 480 & 16.5 & $<.01$ & 1.6 & .03 & .08 & -- \\
\hline $11 / 10 / 93$ & 476,000 & 491 & 13.0 & .03 & 1.7 & .05 & .08 & -- \\
\hline $11 / 19 / 93$ & 553,000 & 431 & 14.0 & .02 & 1.3 & .06 & .05 & -- \\
\hline $11 / 24 / 93$ & 888,000 & 431 & 13.0 & .02 & 1.4 & .02 & .06 & -- \\
\hline $12 / 04 / 93$ & $1,033,000$ & 342 & 12.5 & $<.01$ & 1.1 & .02 & .07 & -- \\
\hline $12 / 09 / 93$ & 977,000 & 368 & 10.0 & .02 & 1.3 & .03 & .07 & -- \\
\hline $12 / 22 / 93$ & $1,062,000$ & 331 & 9.0 & .02 & 1.2 & .03 & .07 & -- \\
\hline $12 / 31 / 93$ & 665,000 & 371 & 8.5 & .03 & 1.3 & .05 & .05 & -- \\
\hline $01 / 06 / 94$ & 529,000 & 391 & 7.5 & .03 & 1.4 & .05 & .05 & -- \\
\hline $01 / 13 / 94$ & 506,000 & 412 & 6.0 & .03 & 1.5 & .06 & .06 & -- \\
\hline $01 / 21 / 94$ & 762,000 & 377 & 4.0 & .02 & 1.3 & .06 & .04 & -- \\
\hline $01 / 27 / 94$ & 648,000 & 344 & 6.5 & .04 & 1.1 & .07 & .04 & -- \\
\hline $02 / 04 / 94$ & 838,000 & 322 & 5.5 & .02 & 1.0 & .06 & .04 & -- \\
\hline $02 / 10 / 94$ & $1,045,000$ & 314 & 6.0 & .06 & 1.2 & .09 & .05 & -- \\
\hline $02 / 17 / 94$ & $1,123,000$ & 287 & 5.5 & .05 & 1.3 & .08 & .04 & -- \\
\hline $02 / 25 / 94$ & $1,094,000$ & 278 & 8.0 & .03 & 1.2 & .04 & .04 & -- \\
\hline 03/04/94 & $1,221,000$ & 316 & 9.0 & .02 & 1.1 & .06 & .04 & -- \\
\hline $03 / 11 / 94$ & $1,279,000$ & 313 & 9.0 & .03 & 1.3 & .06 & .04 & -- \\
\hline $03 / 23 / 94$ & $1,307,000$ & 299 & 12.0 & .03 & 1.4 & .02 & .04 & -- \\
\hline $04 / 01 / 94$ & $1,235,000$ & 313 & 13.0 & .03 & 1.2 & .04 & .04 & -- \\
\hline $04 / 08 / 94$ & $1,162,000$ & 299 & 13.5 & .03 & 1.3 & .05 & .04 & -- \\
\hline $04 / 22 / 94$ & $1,346,000$ & 299 & 18.0 & .03 & .86 & .05 & .03 & -- \\
\hline $04 / 28 / 94$ & $1,473,000$ & 282 & 19.5 & .04 & .99 & .04 & .18 & -- \\
\hline $06 / 24 / 94$ & 493,000 & 403 & 27.5 & $<.01$ & 1.1 & .03 & .07 & -- \\
\hline
\end{tabular}


Table 8. Results of analysis of streamflow, physical properties, and nutrients for water samples collected from three sampling sites in the lower Mississippi River, Louisiana, 1991-2003.-Continued

[Streamflow data from U.S. Army Corps of Engineers, New Orleans District. Physical property data from various collectors. Nutrient data from U.S Geological Survey National Water-Quality Laboratory, Lakewood, Colorado; $\mathrm{ft}^{3} / \mathrm{s}$, cubic feet per second; $\mu \mathrm{S} / \mathrm{cm}$, microsiemens per centimeter at 25 degrees Celsius; ${ }^{\circ} \mathrm{C}$, degrees Celsius; $\mathrm{mg} / \mathrm{L}$, milligrams per liter; <, less than; --, no data]

\begin{tabular}{|c|c|c|c|c|c|c|c|c|}
\hline $\begin{array}{c}\text { Collection } \\
\text { (month/day/ } \\
\text { year) }\end{array}$ & $\begin{array}{c}\text { Streamflow } \\
\left(\mathrm{ft}^{3} / \mathrm{s}\right)\end{array}$ & $\begin{array}{c}\text { Specific } \\
\text { conductance } \\
(\mu \mathrm{S} / \mathrm{cm})\end{array}$ & $\begin{array}{c}\text { Water } \\
\text { temperature } \\
\left({ }^{\circ} \mathrm{C}\right)\end{array}$ & $\begin{array}{c}\text { Nitrite as } \\
\text { nitrogen } \\
\text { (mg/L) }\end{array}$ & $\begin{array}{l}\text { Nitrite plus } \\
\text { nitrate as } \\
\text { nitrogen } \\
\text { (mg/L) }\end{array}$ & $\begin{array}{l}\text { Ammonia } \\
\text { as nitrogen } \\
\text { (mg/L) }\end{array}$ & $\begin{array}{l}\text { Orthophosphate } \\
\text { as phosphorus } \\
(\mathrm{mg} / \mathrm{L})\end{array}$ & $\begin{array}{c}\text { Silica as } \\
\mathrm{SiO}_{2}(\mathrm{mg} / \mathrm{L})\end{array}$ \\
\hline
\end{tabular}

\begin{tabular}{|c|c|c|c|c|c|c|c|c|}
\hline \multicolumn{9}{|c|}{ Mississippi River at Baton Rouge, Louisiana, station 07374000 (fig. 1)—Continued } \\
\hline 07/01/94 & 479,000 & 407 & 29.5 & $<0.01$ & 1.2 & 0.02 & 0.07 & -- \\
\hline 07/07/94 & 546,000 & 429 & 19.5 & $<.01$ & 1.4 & .03 & .08 & -- \\
\hline $07 / 15 / 94$ & 530,000 & 413 & 28.5 & $<.01$ & 1.4 & .05 & .08 & -- \\
\hline $07 / 22 / 94$ & 517,000 & 408 & 29.5 & $<.01$ & 1.7 & .03 & .08 & -- \\
\hline $08 / 05 / 94$ & 459,000 & 446 & 30.0 & $<.01$ & 1.4 & .03 & .09 & -- \\
\hline $08 / 09 / 94$ & 449,000 & 437 & 29.0 & $<.01$ & 1.5 & .02 & .08 & -- \\
\hline $08 / 19 / 94$ & 339,000 & 433 & 29.5 & .01 & .90 & .02 & .07 & -- \\
\hline $08 / 29 / 94$ & 376,000 & 452 & 30.5 & $<.01$ & .49 & .03 & .04 & -- \\
\hline $09 / 08 / 94$ & 343,000 & 444 & 30.5 & $<.01$ & .65 & .03 & .06 & 2.5 \\
\hline $09 / 16 / 94$ & 303,000 & 454 & 29.5 & $<.01$ & .66 & .02 & .06 & 4.2 \\
\hline $09 / 23 / 94$ & 272,000 & 452 & 26.0 & $<.01$ & .59 & .01 & .05 & 4.3 \\
\hline $09 / 30 / 94$ & 255,000 & 450 & 25.1 & .02 & .57 & .03 & .07 & 4.6 \\
\hline $10 / 05 / 94$ & 272,000 & 453 & 25.5 & .01 & .59 & .03 & .07 & 4.3 \\
\hline $10 / 13 / 94$ & 311,000 & 442 & 21.5 & $<.01$ & .79 & .02 & .08 & 5.9 \\
\hline $10 / 21 / 94$ & 257,000 & 422 & 21.0 & $<.01$ & .77 & $<.02$ & .08 & 7.5 \\
\hline $10 / 26 / 94$ & 314,000 & 456 & 21.0 & $<.01$ & .95 & $<.02$ & .08 & 8.5 \\
\hline $11 / 04 / 94$ & 276,000 & 444 & 21.0 & $<.01$ & .91 & $<.02$ & .08 & 15.0 \\
\hline $11 / 10 / 94$ & 358,000 & 435 & 19.0 & $<.01$ & 1.0 & .02 & .07 & 9.9 \\
\hline $11 / 18 / 94$ & 462,000 & 420 & 18.0 & $<.01$ & 1.1 & $<.02$ & .08 & 9.1 \\
\hline $11 / 23 / 94$ & 456,000 & 369 & 17.6 & $<.01$ & 1.0 & $<.02$ & .07 & 9.1 \\
\hline $12 / 01 / 94$ & 455,000 & 365 & 16.0 & .02 & .99 & .02 & .07 & 8.8 \\
\hline $12 / 08 / 94$ & 570,000 & 374 & 16.0 & .02 & 1.0 & .02 & .07 & 9.6 \\
\hline $12 / 15 / 94$ & 662,000 & 401 & 12.0 & .01 & 1.0 & .02 & .06 & 6.9 \\
\hline $12 / 23 / 94$ & 738,000 & 359 & 10.0 & .01 & .97 & .02 & .05 & 6.0 \\
\hline $01 / 05 / 95$ & 422,000 & 383 & 8.0 & .02 & 1.2 & .04 & .06 & 7.3 \\
\hline $01 / 13 / 95$ & 358,000 & 415 & 10.5 & .02 & 1.3 & .08 & .06 & 7.7 \\
\hline $01 / 19 / 95$ & 509,000 & 351 & 7.5 & .02 & 1.0 & .05 & .06 & 6.2 \\
\hline $01 / 27 / 95$ & 862,000 & 328 & 8.0 & .02 & 1.1 & .03 & .06 & 6.2 \\
\hline $02 / 03 / 95$ & 898,000 & 331 & 7.5 & .02 & 1.3 & .03 & .05 & 7.0 \\
\hline $02 / 10 / 95$ & 723,000 & 351 & 8.0 & .01 & 1.4 & .02 & .05 & 7.6 \\
\hline $02 / 17 / 95$ & 550,000 & 366 & 11.0 & .01 & 1.3 & .04 & .05 & 7.3 \\
\hline $02 / 24 / 95$ & 575,000 & 347 & 10.0 & .01 & 1.2 & .04 & .05 & 6.6 \\
\hline $03 / 02 / 95$ & 731,000 & 343 & 8.0 & .02 & 1.2 & .04 & .06 & 6.5 \\
\hline
\end{tabular}


Table 8. Results of analysis of streamflow, physical properties, and nutrients for water samples collected from three sampling sites in the lower Mississippi River, Louisiana, 1991-2003.-Continued

[Streamflow data from U.S. Army Corps of Engineers, New Orleans District. Physical property data from various collectors. Nutrient data from U.S. Geological Survey National Water-Quality Laboratory, Lakewood, Colorado; $\mathrm{ft}^{3} / \mathrm{s}$, cubic feet per second; $\mu \mathrm{S} / \mathrm{cm}$, microsiemens per centimeter at 25 degrees Celsius; ${ }^{\circ} \mathrm{C}$, degrees Celsius; mg/L, milligrams per liter; <, less than; --, no data]

\begin{tabular}{|c|c|c|c|c|c|c|c|c|}
\hline $\begin{array}{c}\text { Collection } \\
\text { (month/day/ } \\
\text { year) }\end{array}$ & $\begin{array}{l}\text { Streamflow } \\
\qquad\left(\mathrm{ft}^{3} / \mathrm{s}\right)\end{array}$ & $\begin{array}{c}\text { Specific } \\
\text { conductance } \\
(\mu \mathrm{S} / \mathrm{cm})\end{array}$ & $\begin{array}{c}\text { Water } \\
\text { temperature } \\
\left({ }^{\circ} \mathrm{C}\right)\end{array}$ & $\begin{array}{l}\text { Nitrite as } \\
\text { nitrogen } \\
\text { (mg/L) }\end{array}$ & $\begin{array}{c}\text { Nitrite plus } \\
\text { nitrate as } \\
\text { nitrogen } \\
\text { (mg/L) }\end{array}$ & $\begin{array}{l}\text { Ammonia } \\
\text { as nitrogen } \\
\text { (mg/L) }\end{array}$ & $\begin{array}{c}\text { Orthophosphate } \\
\text { as phosphorus } \\
\text { (mg/L) }\end{array}$ & $\begin{array}{c}\text { Silica as } \\
\mathrm{SiO}_{2}(\mathrm{mg} / \mathrm{L})\end{array}$ \\
\hline \multicolumn{9}{|c|}{ Mississippi River at Baton Rouge, Louisiana, station 07374000 (fig. 1)—Continued } \\
\hline 03/10/95 & 641,000 & 340 & 13.0 & 0.03 & 1.2 & 0.04 & 0.04 & 8.1 \\
\hline $03 / 15 / 95$ & 781,000 & 343 & 16.8 & .05 & 1.4 & .03 & .04 & 5.5 \\
\hline 03/29/95 & 837,000 & 406 & 16.5 & .01 & 1.3 & $<.02$ & .05 & 6.9 \\
\hline $04 / 05 / 95$ & 587,000 & 413 & 17.0 & .02 & 1.5 & $<.02$ & .06 & 5.4 \\
\hline $04 / 14 / 95$ & 470,000 & 431 & 18.5 & .02 & 1.5 & $<.02$ & .05 & 8.4 \\
\hline $04 / 21 / 95$ & 530,000 & 382 & 20.5 & $<.01$ & 1.7 & $<.02$ & .05 & 5.9 \\
\hline $04 / 28 / 95$ & 753,000 & 377 & 19.0 & $<.01$ & 1.6 & .02 & .06 & 7.8 \\
\hline 05/05/95 & 847,000 & 410 & 19.5 & $<.01$ & 2.2 & .02 & .07 & 6.5 \\
\hline $05 / 15 / 95$ & 891,000 & 405 & 22.5 & $<.01$ & 1.9 & $<.02$ & .06 & 7.6 \\
\hline 05/19/95 & 937,000 & 382 & 23.5 & $<.01$ & 1.6 & .02 & .06 & 6.2 \\
\hline $05 / 26 / 95$ & $1,051,000$ & 371 & 24.0 & $<.01$ & 1.8 & .02 & .08 & 8.3 \\
\hline 06/07/95 & $1,500,000$ & 324 & 24.5 & $<.01$ & 1.4 & $<.02$ & .07 & 7.0 \\
\hline $06 / 15 / 95$ & $1,632,000$ & 360 & 25.5 & $<.01$ & 1.4 & .02 & .07 & 7.4 \\
\hline $06 / 23 / 95$ & $1,535,000$ & 398 & 26.0 & .01 & 1.6 & .04 & .09 & 7.7 \\
\hline 07/03/95 & $1,180,000$ & 407 & 28.0 & $<.01$ & 1.7 & .02 & .08 & 8.0 \\
\hline 07/06/95 & $1,042,000$ & 434 & 27.0 & .01 & 1.7 & .02 & .08 & 8.2 \\
\hline 07/20/95 & 779,000 & 436 & 30.5 & $<.01$ & 1.9 & .02 & .09 & 11 \\
\hline $07 / 27 / 95$ & 562,000 & 430 & 30.6 & $<.01$ & 1.6 & .02 & .09 & 8.5 \\
\hline 08/02/95 & 487,000 & 431 & 30.0 & $<.01$ & 1.6 & .06 & .10 & 7.9 \\
\hline $08 / 11 / 95$ & 483,000 & 451 & 30.5 & $<.01$ & 1.2 & .05 & .09 & 6.7 \\
\hline 08/18/95 & 657,000 & 388 & 30.5 & $<.01$ & 1.0 & $<.02$ & .08 & 6.0 \\
\hline $08 / 25 / 95$ & 528,000 & 445 & 32.0 & $<.01$ & 1.0 & $<.02$ & .08 & 6.2 \\
\hline 09/01/95 & 439,000 & 495 & 29.5 & $<.01$ & 1.1 & $<.02$ & .10 & 8.3 \\
\hline 09/07/95 & 373,000 & 502 & 29.5 & $<.01$ & .95 & $<.02$ & .10 & 9.0 \\
\hline $09 / 15 / 95$ & 298,000 & 532 & 29.0 & $<.01$ & .88 & $<.02$ & .09 & 9.0 \\
\hline $09 / 22 / 95$ & 316,000 & 537 & 27.0 & $<.01$ & .76 & $<.02$ & .10 & 8.8 \\
\hline 09/28/95 & 293,000 & 528 & 25.0 & $<.01$ & .59 & $<.02$ & .08 & 7.3 \\
\hline 01/12/96 & 400,000 & 428 & 5.0 & .01 & 1.2 & .07 & .07 & 7.1 \\
\hline 01/30/96 & 771,000 & 397 & 7.5 & .02 & 1.2 & .06 & .06 & 6.8 \\
\hline 02/16/96 & 824,000 & 347 & 6.5 & .02 & 1.5 & .04 & .05 & 6.7 \\
\hline 02/29/96 & 598,000 & 392 & 8.5 & .03 & 1.4 & .02 & .05 & 7.2 \\
\hline 03/08/96 & 641,000 & 375 & 9.0 & .03 & 1.6 & .04 & .05 & 7.3 \\
\hline 03/15/96 & 812,000 & 387 & 9.5 & .03 & 1.6 & .05 & .06 & 6.8 \\
\hline
\end{tabular}


Table 8. Results of analysis of streamflow, physical properties, and nutrients for water samples collected from three sampling sites in the lower Mississippi River, Louisiana, 1991-2003.-Continued

[Streamflow data from U.S. Army Corps of Engineers, New Orleans District. Physical property data from various collectors. Nutrient data from U.S Geological Survey National Water-Quality Laboratory, Lakewood, Colorado; $\mathrm{ft}^{3} / \mathrm{s}$, cubic feet per second; $\mu \mathrm{S} / \mathrm{cm}$, microsiemens per centimeter at 25 degrees Celsius; ${ }^{\circ} \mathrm{C}$, degrees Celsius; $\mathrm{mg} / \mathrm{L}$, milligrams per liter; <, less than; --, no data]

\begin{tabular}{|c|c|c|c|c|c|c|c|c|}
\hline $\begin{array}{c}\text { Collection } \\
\text { (month/day/ } \\
\text { year) }\end{array}$ & $\begin{array}{c}\text { Streamflow } \\
\left(\mathrm{ft}^{3} / \mathrm{s}\right)\end{array}$ & $\begin{array}{c}\text { Specific } \\
\text { conductance } \\
(\mu \mathrm{S} / \mathrm{cm})\end{array}$ & $\begin{array}{c}\text { Water } \\
\text { temperature } \\
\left({ }^{\circ} \mathrm{C}\right)\end{array}$ & $\begin{array}{l}\text { Nitrite as } \\
\text { nitrogen } \\
\text { (mg/L) }\end{array}$ & $\begin{array}{c}\text { Nitrite plus } \\
\text { nitrate as } \\
\text { nitrogen } \\
\text { (mg/L) }\end{array}$ & $\begin{array}{l}\text { Ammonia } \\
\text { as nitrogen } \\
\text { (mg/L) }\end{array}$ & $\begin{array}{l}\text { Orthophosphate } \\
\text { as phosphorus } \\
\text { (mg/L) }\end{array}$ & $\begin{array}{c}\text { Silica as } \\
\mathrm{SiO}_{2}(\mathrm{mg} / \mathrm{L})\end{array}$ \\
\hline \multicolumn{9}{|c|}{ Mississippi River at Baton Rouge, Louisiana, station 07374000 (fig. 1)—Continued } \\
\hline $04 / 12 / 96$ & 889,000 & 388 & 12.0 & 0.02 & 1.5 & 0.03 & 0.04 & 6.4 \\
\hline $04 / 26 / 96$ & 617,000 & 391 & 18.5 & .03 & 1.5 & $<.02$ & .06 & 6.6 \\
\hline 05/03/96 & 963,000 & 382 & 18.0 & $<.01$ & 1.2 & .02 & .06 & 5.5 \\
\hline 05/10/96 & $1,122,000$ & -- & -- & $<.01$ & 1.4 & $<.02$ & .06 & 6.1 \\
\hline 05/17/96 & $1,198,000$ & 334 & 21.0 & $<.01$ & 1.4 & $<.02$ & .06 & 6.1 \\
\hline $05 / 23 / 96$ & $1,294,000$ & 319 & 23.2 & $<.01$ & 1.4 & $<.02$ & .05 & 6.3 \\
\hline $05 / 31 / 96$ & $1,390,000$ & 321 & 24.3 & .01 & 1.2 & .03 & .07 & 7.3 \\
\hline 06/06/96 & $1,423,000$ & 326 & 25.1 & $<.01$ & 1.2 & .03 & .07 & 6.7 \\
\hline $07 / 18 / 96$ & 543,000 & 471 & 28.5 & $<.01$ & 2.7 & .04 & .12 & 8.9 \\
\hline 08/02/96 & 648,000 & -- & -- & $<.01$ & 1.9 & $<.02$ & .10 & 5.8 \\
\hline 08/09/96 & 571,000 & 397 & 29.0 & .01 & 1.7 & .03 & .10 & 7.2 \\
\hline 08/30/96 & 402,000 & 472 & 28.5 & $<.01$ & 1.5 & $<.02$ & .10 & 7.0 \\
\hline 09/30/96 & 402,000 & 462 & 23.5 & $<.01$ & .80 & $<.02$ & .06 & 11 \\
\hline $10 / 30 / 96$ & 348,000 & 520 & 19.5 & .01 & .80 & $<.02$ & .07 & 7.2 \\
\hline $11 / 21 / 96$ & 816,000 & 400 & 13.5 & .02 & .78 & $<.02$ & .07 & 6.0 \\
\hline $12 / 11 / 96$ & $1,048,000$ & 340 & 9.5 & .03 & .97 & .02 & .05 & 7.1 \\
\hline $12 / 31 / 96$ & $1,040,000$ & 297 & 7.5 & .03 & 1.0 & .03 & .06 & 6.7 \\
\hline 03/05/97 & $1,081,000$ & 444 & 12.1 & .03 & 1.6 & .13 & .38 & 7.5 \\
\hline $03 / 22 / 97$ & $1,866,000$ & 254 & 14.0 & .03 & 1.2 & .02 & .05 & 5.8 \\
\hline $03 / 27 / 97$ & $1,915,000$ & 263 & 14.5 & .03 & 1.3 & $<.02$ & .04 & 6.5 \\
\hline 04/03/97 & $1,831,000$ & 291 & 15.5 & .02 & 1.4 & $<.02$ & .05 & 6.5 \\
\hline 04/17/97 & $1,346,000$ & 320 & 16.5 & .02 & 1.2 & $<.02$ & .06 & 6.1 \\
\hline 04/30/97 & $1,115,000$ & 360 & 16.0 & .02 & 1.5 & $<.02$ & .06 & 6.8 \\
\hline 05/16/97 & 994,000 & 412 & 20.5 & $<.01$ & 1.3 & $<.02$ & .05 & 6.5 \\
\hline $05 / 27 / 97$ & 706,000 & 436 & 23.8 & $<.01$ & 1.7 & $<.02$ & .06 & 6.6 \\
\hline 06/06/97 & 797,000 & 454 & 24.0 & $<.01$ & 1.6 & $<.02$ & .06 & 5.0 \\
\hline $06 / 27 / 97$ & $1,094,000$ & 379 & 26.5 & $<.01$ & 1.7 & $<.02$ & .06 & 5.6 \\
\hline 07/01/97 & $1,104,000$ & 375 & 27.5 & $<.01$ & 1.7 & $<.02$ & .07 & 5.9 \\
\hline 07/14/97 & 757,000 & 415 & 29.3 & $<.01$ & 1.5 & $<.02$ & .07 & 6.4 \\
\hline $07 / 25 / 97$ & 481,000 & 480 & 30.9 & $<.01$ & 1.7 & .03 & .08 & 7.3 \\
\hline 08/14/97 & 365,000 & 507 & 30.5 & $<.01$ & 1.0 & $<.02$ & .06 & 3.2 \\
\hline $12 / 10 / 97$ & 356,000 & 572 & 11.5 & $<.01$ & 1.1 & $<.02$ & .06 & 6.3 \\
\hline $01 / 14 / 98$ & 742,000 & 386 & 10.0 & .01 & .79 & $<.02$ & .04 & 6.6 \\
\hline
\end{tabular}


Table 8. Results of analysis of streamflow, physical properties, and nutrients for water samples collected from three sampling sites in the lower Mississippi River, Louisiana, 1991-2003.-Continued

[Streamflow data from U.S. Army Corps of Engineers, New Orleans District. Physical property data from various collectors. Nutrient data from U.S. Geological Survey National Water-Quality Laboratory, Lakewood, Colorado; $\mathrm{ft}^{3} / \mathrm{s}$, cubic feet per second; $\mu \mathrm{S} / \mathrm{cm}$, microsiemens per centimeter at 25 degrees Celsius; ${ }^{\circ} \mathrm{C}$, degrees Celsius; $\mathrm{mg} / \mathrm{L}$, milligrams per liter; <, less than; --, no data]

\begin{tabular}{|c|c|c|c|c|c|c|c|c|}
\hline $\begin{array}{c}\text { Collection } \\
\text { (month/day/ } \\
\text { year) }\end{array}$ & $\begin{array}{c}\text { Streamflow } \\
\left(\mathrm{ft}^{3} / \mathrm{s}\right)\end{array}$ & $\begin{array}{c}\text { Specific } \\
\text { conductance } \\
(\mu \mathrm{S} / \mathrm{cm})\end{array}$ & $\begin{array}{c}\text { Water } \\
\text { temperature } \\
\left({ }^{\circ} \mathrm{C}\right)\end{array}$ & $\begin{array}{l}\text { Nitrite as } \\
\text { nitrogen } \\
\text { (mg/L) }\end{array}$ & $\begin{array}{c}\text { Nitrite plus } \\
\text { nitrate as } \\
\text { nitrogen } \\
\text { (mg/L) }\end{array}$ & $\begin{array}{l}\text { Ammonia } \\
\text { as nitrogen } \\
\text { (mg/L) }\end{array}$ & $\begin{array}{l}\text { Orthophosphate } \\
\text { as phosphorus } \\
\text { (mg/L) }\end{array}$ & $\begin{array}{c}\text { Silica as } \\
\mathrm{SiO}_{2}(\mathrm{mg} / \mathrm{L})\end{array}$ \\
\hline \multicolumn{9}{|c|}{ Mississippi River at Baton Rouge, Louisiana, station 07374000 (fig. 1)—Continued } \\
\hline 02/04/98 & 827,000 & 349 & 8.5 & $<0.01$ & 1.2 & $<0.02$ & 0.05 & 6.4 \\
\hline 02/19/98 & 903,000 & 389 & 9.0 & $<.01$ & 1.2 & .04 & .05 & 6.2 \\
\hline 03/04/98 & $1,002,000$ & 316 & 10.5 & $<.01$ & 1.4 & $<.02$ & .06 & 6.7 \\
\hline 03/10/98 & $1,009,000$ & 340 & 11.0 & .01 & 1.6 & .02 & .06 & 6.7 \\
\hline 03/20/98 & $1,032,000$ & 361 & 10.5 & .01 & 1.7 & .03 & .05 & 6.4 \\
\hline 03/27/98 & $1,126,000$ & 342 & 11.5 & .02 & 1.6 & .05 & .05 & 6.4 \\
\hline 04/10/98 & $1,230,000$ & 350 & 16.5 & $<.01$ & 1.8 & .03 & .06 & 6.6 \\
\hline 04/17/98 & $1,178,000$ & 366 & 17.0 & .01 & 2.2 & .03 & .07 & 7.4 \\
\hline 04/22/98 & $1,192,000$ & 394 & 17.0 & .02 & 2.6 & .03 & .01 & 7.3 \\
\hline 05/08/98 & $1,445,000$ & 375 & 20.0 & .03 & 1.8 & .03 & .07 & 6.5 \\
\hline 07/24/98 & 842,000 & -- & -- & $<.01$ & 2.3 & .04 & .10 & 10 \\
\hline 08/07/98 & 610,000 & 408 & 29.5 & $<.01$ & 2.0 & .08 & .11 & 8.9 \\
\hline 08/13/98 & 587,000 & 343 & 29.0 & $<.01$ & 1.4 & .08 & .10 & 7.5 \\
\hline 08/27/98 & 443,000 & 407 & 30.5 & $<.01$ & 1.4 & $<.02$ & .09 & 8.0 \\
\hline 09/18/98 & 265,000 & 464 & 28.3 & $<.01$ & 1.2 & $<.02$ & .09 & 6.4 \\
\hline $10 / 02 / 98$ & 291,000 & 510 & 28.5 & $<.01$ & 1.0 & $<.02$ & .11 & 6.8 \\
\hline $10 / 23 / 98$ & 496,000 & 378 & 20.4 & $<.01$ & .93 & $<.02$ & .07 & 6.9 \\
\hline $10 / 30 / 98$ & 490,000 & 390 & 20.3 & $<.01$ & .91 & $<.02$ & .09 & 7.8 \\
\hline $11 / 10 / 98$ & 398,000 & 414 & 17.6 & $<.01$ & 1.3 & .04 & .10 & 8.1 \\
\hline $12 / 23 / 98$ & 564,000 & 470 & 10.6 & .02 & 1.5 & .04 & .09 & 9.3 \\
\hline 01/05/99 & 490,000 & 445 & 6.7 & .01 & 1.3 & .04 & .06 & 7.5 \\
\hline 02/03/99 & $1,170,000$ & 333 & 9.3 & .02 & 1.5 & .04 & .05 & 6.3 \\
\hline 02/23/99 & $1,347,000$ & 326 & 10.7 & .03 & 1.7 & $<.02$ & .07 & 7.8 \\
\hline 03/12/99 & 789,000 & 418 & 10.5 & .02 & 2.1 & .02 & .06 & 7.5 \\
\hline $03 / 26 / 99$ & $1,216,000$ & 344 & 10.8 & .01 & 1.4 & $<.02$ & .05 & 6.7 \\
\hline 04/23/99 & 788,000 & 444 & 19.0 & .01 & 1.4 & .03 & .07 & 5.1 \\
\hline 04/30/99 & 949,000 & 465 & 18.1 & $<.01$ & 2.2 & .06 & .06 & 7.4 \\
\hline 05/07/99 & $1,129,000$ & 457 & 19.5 & $<.01$ & 2.8 & .04 & .07 & 7.2 \\
\hline 05/17/99 & $1,228,000$ & 397 & 22.0 & $<.01$ & 2.7 & .02 & .06 & 6.9 \\
\hline $05 / 25 / 99$ & $1,043,000$ & 390 & 23.4 & $<.01$ & 2.1 & .02 & .07 & 6.4 \\
\hline 06/03/99 & 868,000 & 448 & 26.5 & $<.01$ & 2.6 & .03 & .08 & 7.6 \\
\hline 06/10/99 & 806,000 & 469 & 26.5 & $<.01$ & 2.9 & $<.02$ & .08 & 7.8 \\
\hline 06/18/99 & 831,000 & 454 & 27.4 & $<.01$ & 2.7 & $<.02$ & .08 & 7.7 \\
\hline 06/25/99 & 668,000 & 463 & 26.8 & $<.01$ & 2.8 & .03 & .09 & 8.8 \\
\hline
\end{tabular}


Table 8. Results of analysis of streamflow, physical properties, and nutrients for water samples collected from three sampling sites in the lower Mississippi River, Louisiana, 1991-2003.-Continued

[Streamflow data from U.S. Army Corps of Engineers, New Orleans District. Physical property data from various collectors. Nutrient data from U.S Geological Survey National Water-Quality Laboratory, Lakewood, Colorado; $\mathrm{ft}^{3} / \mathrm{s}$, cubic feet per second; $\mu \mathrm{S} / \mathrm{cm}$, microsiemens per centimeter at 25 degrees Celsius; ${ }^{\circ} \mathrm{C}$, degrees Celsius; $\mathrm{mg} / \mathrm{L}$, milligrams per liter; <, less than; --, no data]

\begin{tabular}{|c|c|c|c|c|c|c|c|c|}
\hline $\begin{array}{c}\text { Collection } \\
\text { (month/day/ } \\
\text { year) }\end{array}$ & $\begin{array}{l}\text { Streamflow } \\
\left(\mathrm{ft}^{3} / \mathrm{s}\right)\end{array}$ & $\begin{array}{c}\text { Specific } \\
\text { conductance } \\
(\mu \mathrm{S} / \mathrm{cm})\end{array}$ & $\begin{array}{c}\text { Water } \\
\text { temperature } \\
\left({ }^{\circ} \mathrm{C}\right)\end{array}$ & $\begin{array}{l}\text { Nitrite as } \\
\text { nitrogen } \\
\text { (mg/L) }\end{array}$ & $\begin{array}{c}\text { Nitrite plus } \\
\text { nitrate as } \\
\text { nitrogen } \\
\text { (mg/L) }\end{array}$ & $\begin{array}{l}\text { Ammonia } \\
\text { as nitrogen } \\
\text { (mg/L) }\end{array}$ & $\begin{array}{l}\text { Orthophosphate } \\
\text { as phosphorus } \\
\text { (mg/L) }\end{array}$ & $\begin{array}{c}\text { Silica as } \\
\mathrm{SiO}_{2}(\mathrm{mg} / \mathrm{L})\end{array}$ \\
\hline \multicolumn{9}{|c|}{ Mississippi River at Baton Rouge, Louisiana, station 07374000 (fig. 1)—Continued } \\
\hline 07/09/99 & 779,000 & 465 & 29.3 & $<0.01$ & 2.7 & $<0.02$ & 0.10 & -- \\
\hline 07/16/99 & 780,000 & -- & -- & $<.01$ & 2.2 & $<.02$ & .09 & 8.7 \\
\hline 08/06/99 & 444,000 & 412 & 31.2 & $<.01$ & 1.9 & $<.02$ & .09 & 8.7 \\
\hline 08/17/99 & 401,000 & 480 & 31.8 & $<.01$ & 2.2 & $<.02$ & .11 & 9.4 \\
\hline 08/23/99 & 363,000 & 480 & 31.4 & $<.01$ & 2.0 & $<.02$ & .10 & 9.2 \\
\hline 09/02/99 & 269,000 & 480 & 32.8 & $<.01$ & 1.5 & .03 & .08 & 9.0 \\
\hline 09/27/99 & 207,000 & 573 & 26.8 & $<.01$ & .69 & .03 & .06 & 7.2 \\
\hline $10 / 04 / 99$ & 213,000 & 577 & 25.4 & $<.01$ & .85 & .02 & .08 & 7.5 \\
\hline $10 / 20 / 99$ & 250,000 & 548 & 22.8 & $<.01$ & .89 & $<.02$ & .08 & 8.0 \\
\hline $11 / 01 / 99$ & 196,000 & 552 & 19.9 & $<.01$ & .81 & .02 & .06 & 7.5 \\
\hline $11 / 19 / 99$ & 231,000 & 495 & 18.2 & $<.01$ & .81 & .03 & .06 & 6.6 \\
\hline $12 / 06 / 99$ & 227,000 & 581 & 15.1 & .01 & .93 & .03 & .05 & 7.5 \\
\hline $01 / 13 / 00$ & 395,000 & 583 & 11.3 & .02 & 1.3 & .10 & .05 & 8.7 \\
\hline $02 / 14 / 00$ & 205,000 & 543 & 9.5 & .02 & 1.1 & .10 & .05 & 8.2 \\
\hline 05/02/00 & 589,000 & 414 & 19.5 & $<.01$ & 1.3 & $<.01$ & .05 & 5.4 \\
\hline 05/12/00 & 601,000 & 424 & 23.2 & $<.01$ & 1.3 & $<.01$ & .06 & 4.9 \\
\hline $05 / 22 / 00$ & 434,000 & 434 & 25.0 & $<.01$ & 1.3 & $<.01$ & .06 & 5.6 \\
\hline 06/20/00 & 435,000 & -- & -- & $<.01$ & 2.1 & $<.01$ & .09 & 6.4 \\
\hline $06 / 29 / 00$ & 777,000 & 416 & 27.5 & $<.01$ & 2.1 & $<.01$ & .09 & 6.5 \\
\hline 07/07/00 & 867,000 & 410 & 28.7 & $<.01$ & 2.5 & .04 & .10 & 7.9 \\
\hline $07 / 18 / 00$ & -- & -- & 27.2 & $<.01$ & 2.8 & $<.01$ & .10 & 8.4 \\
\hline 08/03/00 & 425,000 & 458 & 28.5 & $<.01$ & 2.5 & $<.01$ & .12 & 8.2 \\
\hline 08/11/00 & 344,000 & 476 & 30.0 & $<.01$ & 1.7 & $<.01$ & .10 & 7.4 \\
\hline $08 / 21 / 00$ & 396,000 & 447 & 30.5 & $<.01$ & .91 & $<.01$ & .07 & .90 \\
\hline $08 / 29 / 00$ & 282,000 & 487 & 31.5 & $<.01$ & .92 & $<.01$ & .07 & 5.1 \\
\hline $09 / 11 / 00$ & 251,000 & -- & 28.3 & $<.01$ & .83 & $<.01$ & .08 & 2.6 \\
\hline 09/27/00 & 231,000 & 481 & 25.2 & $<.01$ & .55 & $<.01$ & .08 & 3.0 \\
\hline $10 / 10 / 00$ & 299,000 & 505 & 20.0 & .03 & .88 & $<.02$ & .09 & 5.1 \\
\hline $10 / 19 / 00$ & 277,000 & 498 & 20.1 & .05 & .94 & $<.02$ & .08 & 6.0 \\
\hline $11 / 20 / 00$ & 318,000 & 534 & 17.9 & .05 & .88 & .03 & .06 & 6.1 \\
\hline $12 / 04 / 00$ & 371,000 & 440 & 10.0 & .01 & 1.0 & .05 & .08 & 6.7 \\
\hline 01/05/01 & 444,000 & 412 & 2.5 & .01 & 1.3 & .09 & .06 & 6.0 \\
\hline $02 / 23 / 01$ & 989,000 & 369 & 9.9 & .03 & 1.7 & .09 & .06 & 6.8 \\
\hline
\end{tabular}


Table 8. Results of analysis of streamflow, physical properties, and nutrients for water samples collected from three sampling sites in the lower Mississippi River, Louisiana, 1991-2003.-Continued

[Streamflow data from U.S. Army Corps of Engineers, New Orleans District. Physical property data from various collectors. Nutrient data from U.S. Geological Survey National Water-Quality Laboratory, Lakewood, Colorado; $\mathrm{ft}^{3} / \mathrm{s}$, cubic feet per second; $\mu \mathrm{S} / \mathrm{cm}$, microsiemens per centimeter at 25 degrees Celsius; ${ }^{\circ} \mathrm{C}$, degrees Celsius; mg/L, milligrams per liter; <, less than; --, no data]

\begin{tabular}{|c|c|c|c|c|c|c|c|c|}
\hline $\begin{array}{c}\text { Collection } \\
\text { (month/day/ } \\
\text { year) }\end{array}$ & $\begin{array}{c}\text { Streamflow } \\
\left(\mathrm{ft}^{3} / \mathrm{s}\right)\end{array}$ & $\begin{array}{c}\text { Specific } \\
\text { conductance } \\
(\mu \mathrm{S} / \mathrm{cm})\end{array}$ & $\begin{array}{c}\text { Water } \\
\text { temperature } \\
\left({ }^{\circ} \mathrm{C}\right)\end{array}$ & $\begin{array}{c}\text { Nitrite as } \\
\text { nitrogen } \\
\text { (mg/L) }\end{array}$ & $\begin{array}{c}\text { Nitrite plus } \\
\text { nitrate as } \\
\text { nitrogen } \\
\text { (mg/L) }\end{array}$ & $\begin{array}{l}\text { Ammonia } \\
\text { as nitrogen } \\
(\mathrm{mg} / \mathrm{L})\end{array}$ & $\begin{array}{c}\text { Orthophosphate } \\
\text { as phosphorus } \\
\text { (mg/L) }\end{array}$ & $\begin{array}{c}\text { Silica as } \\
\mathrm{SiO}_{2}(\mathrm{mg} / \mathrm{L})\end{array}$ \\
\hline \multicolumn{9}{|c|}{ Mississippi River at Baton Rouge, Louisiana, station 07374000 (fig. 1)—Continued } \\
\hline 03/06/01 & $1,332,000$ & 320 & 9.9 & 0.03 & 1.6 & 0.02 & 0.04 & 6.2 \\
\hline 03/23/01 & 909,000 & 351 & 12.0 & .03 & 1.7 & .04 & .05 & 6.3 \\
\hline 04/06/01 & 855,000 & 380 & 13.4 & .03 & 2.0 & $<.02$ & .06 & 6.6 \\
\hline $05 / 03 / 01$ & 780,000 & 419 & 18.9 & .01 & 2.9 & $<.02$ & .08 & 7.4 \\
\hline $05 / 17 / 01$ & 567,000 & 430 & 23.5 & .01 & 2.9 & $<.02$ & .11 & 9.3 \\
\hline $05 / 24 / 01$ & 626,000 & 428 & 24.2 & .01 & 2.9 & $<.02$ & .10 & 9.8 \\
\hline $05 / 31 / 01$ & 876,000 & 428 & 23.7 & .01 & 2.7 & $<.02$ & .09 & 7.9 \\
\hline 06/08/01 & 984,000 & 423 & 23.3 & .01 & 2.0 & $<.02$ & .07 & 6.6 \\
\hline 07/02/01 & 660,000 & 460 & 27.3 & .01 & 2.8 & $<.01$ & .02 & 8.4 \\
\hline $07 / 16 / 01$ & 517,000 & 451 & 29.8 & .01 & 2.5 & $<.02$ & .10 & 9.0 \\
\hline $07 / 30 / 01$ & 382,000 & 429 & 29.9 & .01 & 1.9 & $<.02$ & .08 & 7.9 \\
\hline 08/13/01 & 477,000 & 433 & 29.7 & $<.01$ & 1.3 & .03 & .09 & 5.3 \\
\hline 08/28/01 & 305,000 & 419 & 30.0 & .01 & .93 & $<.02$ & .09 & 4.6 \\
\hline 09/18/01 & 275,000 & 440 & 28.0 & $<.01$ & .70 & $<.02$ & .08 & 4.7 \\
\hline $10 / 01 / 01$ & 264,000 & 486 & 24.4 & $<.01$ & .17 & $<.01$ & .02 & 3.0 \\
\hline $10 / 16 / 01$ & 307,000 & 474 & 20.3 & $<.01$ & .85 & $<.02$ & .09 & 6.4 \\
\hline $10 / 31 / 01$ & 437,000 & 437 & 18.2 & $<.01$ & .91 & $<.02$ & .09 & 5.9 \\
\hline $11 / 14 / 01$ & 346,000 & 473 & 16.7 & $<.01$ & 1.5 & $<.02$ & .09 & 7.2 \\
\hline $11 / 28 / 01$ & 271,000 & 537 & 16.8 & .01 & 1.6 & .03 & .08 & 7.9 \\
\hline $12 / 10 / 01$ & 940,000 & 316 & 13.1 & $<.01$ & .97 & $<.02$ & .05 & 5.5 \\
\hline $01 / 03 / 02$ & $1,103,000$ & 285 & 7.0 & .01 & 1.0 & $<.02$ & .06 & 7.0 \\
\hline $01 / 17 / 02$ & 417,000 & 354 & 7.3 & .01 & 1.3 & .04 & .05 & 7.7 \\
\hline $02 / 04 / 02$ & -- & 319 & 10.9 & .02 & 1.2 & .03 & .05 & 5.7 \\
\hline $02 / 28 / 02$ & 600,000 & 359 & 9.8 & .01 & 1.7 & .02 & .06 & 5.9 \\
\hline $03 / 11 / 02$ & 516,000 & 428 & 9.7 & .01 & 1.8 & $<.02$ & .05 & 5.6 \\
\hline $03 / 28 / 02$ & $1,102,000$ & 316 & -- & .03 & 1.5 & .02 & .05 & 4.6 \\
\hline $04 / 12 / 02$ & $1,375,000$ & 275 & 14.6 & .02 & .99 & $<.02$ & .03 & 5.4 \\
\hline $05 / 02 / 02$ & 939,000 & 370 & 21.3 & $<.01$ & 1.8 & $<.02$ & .07 & 5.2 \\
\hline $05 / 15 / 02$ & $1,107,000$ & 311 & 20.3 & $<.01$ & 1.5 & $<.02$ & .06 & 5.7 \\
\hline $06 / 05 / 02$ & $1,503,000$ & 306 & 23.4 & .01 & 1.5 & $<.02$ & .06 & 6.2 \\
\hline $07 / 02 / 02$ & 628,000 & 389 & 27.8 & $<.01$ & 1.9 & $<.02$ & .09 & 7.2 \\
\hline $07 / 25 / 02$ & 406,000 & 440 & 29.4 & $<.01$ & 1.3 & $<.02$ & .08 & 4.2 \\
\hline 08/21/02 & 295,000 & 469 & 29.5 & $<.01$ & .61 & $<.02$ & .07 & 4.5 \\
\hline
\end{tabular}


Table 8. Results of analysis of streamflow, physical properties, and nutrients for water samples collected from three sampling sites in the lower Mississippi River, Louisiana, 1991-2003.-Continued

[Streamflow data from U.S. Army Corps of Engineers, New Orleans District. Physical property data from various collectors. Nutrient data from U.S Geological Survey National Water-Quality Laboratory, Lakewood, Colorado; $\mathrm{ft}^{3} / \mathrm{s}$, cubic feet per second; $\mu \mathrm{S} / \mathrm{cm}$, microsiemens per centimeter at 25 degrees Celsius; ${ }^{\circ} \mathrm{C}$, degrees Celsius; $\mathrm{mg} / \mathrm{L}$, milligrams per liter; <, less than; --, no data]

\begin{tabular}{|c|c|c|c|c|c|c|c|c|}
\hline $\begin{array}{c}\text { Collection } \\
\text { (month/day/ } \\
\text { year) }\end{array}$ & $\begin{array}{l}\text { Streamflow } \\
\left(\mathrm{ft}^{3} / \mathrm{s}\right)\end{array}$ & $\begin{array}{c}\text { Specific } \\
\text { conductance } \\
(\mu \mathrm{S} / \mathrm{cm})\end{array}$ & $\begin{array}{c}\text { Water } \\
\text { temperature } \\
\left({ }^{\circ} \mathrm{C}\right)\end{array}$ & $\begin{array}{l}\text { Nitrite as } \\
\text { nitrogen } \\
\text { (mg/L) }\end{array}$ & $\begin{array}{c}\text { Nitrite plus } \\
\text { nitrate as } \\
\text { nitrogen } \\
\text { (mg/L) }\end{array}$ & $\begin{array}{l}\text { Ammonia } \\
\text { as nitrogen } \\
\text { (mg/L) }\end{array}$ & $\begin{array}{c}\text { Orthophosphate } \\
\text { as phosphorus } \\
\text { (mg/L) }\end{array}$ & $\begin{array}{c}\text { Silica as } \\
\mathrm{SiO}_{2}(\mathrm{mg} / \mathrm{L})\end{array}$ \\
\hline \multicolumn{9}{|c|}{ Mississippi River at Baton Rouge, Louisiana, station 07374000 (fig. 1)—Continued } \\
\hline 09/17/02 & 257,000 & 460 & 24.0 & 0.01 & 0.95 & 0.03 & 0.09 & 6.3 \\
\hline $10 / 16 / 02$ & 421,000 & -- & 22.1 & $<.01$ & .54 & $<.02$ & .07 & 5.6 \\
\hline $11 / 19 / 02$ & 500,000 & 425 & 15.1 & .01 & 1.2 & $<.02$ & .08 & 7.0 \\
\hline $12 / 18 / 02$ & 363,000 & 395 & 10.5 & .01 & 1.0 & $<.02$ & .05 & 6.5 \\
\hline $01 / 15 / 03$ & 738,000 & 347 & 6.7 & $<.01$ & 1.2 & .03 & .05 & 6.1 \\
\hline $02 / 20 / 03$ & 563,000 & 400 & 7.8 & .01 & 1.2 & .03 & .03 & 5.7 \\
\hline $03 / 13 / 03$ & $1,257,000$ & 238 & 8.9 & .01 & .90 & .03 & .03 & 5.4 \\
\hline 04/03/03 & 741,000 & 438 & 15.0 & .04 & 1.5 & $<.02$ & .04 & 4.7 \\
\hline $05 / 05 / 03$ & 644,000 & 425 & 21.0 & $<.01$ & 1.1 & $<.02$ & .04 & -- \\
\hline 05/06/03 & 646,000 & 400 & 22.0 & $<.01$ & 1.1 & $<.02$ & .05 & 3.9 \\
\hline 05/07/03 & 642,000 & 405 & 22.1 & $<.01$ & 1.1 & $<.02$ & .05 & -- \\
\hline 05/08/03 & 641,000 & 411 & 22.5 & $<.01$ & 1.1 & $<.02$ & .05 & 4.1 \\
\hline $05 / 12 / 03$ & -- & 400 & 22.3 & $<.01$ & 1.2 & $<.02$ & .06 & 4.3 \\
\hline $05 / 13 / 03$ & 849,000 & 396 & 22.4 & $<.01$ & 1.2 & $<.02$ & .06 & -- \\
\hline 05/16/03 & $1,014,000$ & 376 & 22.3 & $<.01$ & 1.1 & .08 & .04 & 5.0 \\
\hline 05/19/03 & $1,127,000$ & 316 & 22.6 & $<.01$ & 1.2 & $<.02$ & .06 & 4.9 \\
\hline $05 / 20 / 03$ & $1,167,000$ & 317 & 22.4 & $<.01$ & 1.1 & $<.02$ & .06 & 5.1 \\
\hline $05 / 21 / 03$ & $1,191,000$ & 318 & 22.7 & $<.01$ & 1.2 & $<.02$ & .05 & -- \\
\hline $05 / 22 / 03$ & $1,221,000$ & 321 & 22.6 & $<.01$ & 1.2 & $<.02$ & .06 & 5.2 \\
\hline $05 / 28 / 03$ & $1,401,000$ & 315 & 22.4 & .01 & 1.4 & $<.02$ & .06 & 5.8 \\
\hline $06 / 04 / 03$ & $1,416,000$ & 322 & 23.9 & .01 & 1.7 & $<.02$ & .07 & -- \\
\hline 06/09/03 & $1,112,000$ & 325 & 23.9 & $<.01$ & 1.5 & $<.02$ & .07 & 6.2 \\
\hline 06/10/03 & $1,051,000$ & 323 & 24.6 & .01 & 1.5 & $<.02$ & .07 & -- \\
\hline 06/11/03 & 935,000 & 329 & 24.3 & .01 & 1.5 & $<.02$ & .07 & 6.3 \\
\hline $06 / 12 / 03$ & 878,000 & 339 & 24.4 & $<.01$ & 1.5 & $<.02$ & .08 & -- \\
\hline $06 / 13 / 03$ & 832,000 & 329 & 24.5 & .01 & 1.5 & $<.02$ & .07 & 6.5 \\
\hline 06/16/03 & -- & 321 & 24.1 & .01 & 1.6 & $<.02$ & .07 & -- \\
\hline 06/17/03 & 779,000 & 335 & 24.3 & $<.01$ & 1.7 & $<.02$ & .07 & 5.8 \\
\hline 06/18/03 & 804,000 & 330 & 24.4 & $<.01$ & 1.7 & $<.02$ & .07 & -- \\
\hline 06/19/03 & 833,000 & 341 & 24.0 & $<.01$ & 1.7 & $<.02$ & .07 & 5.8 \\
\hline $06 / 23 / 03$ & 907,000 & 359 & 25.6 & $<.01$ & 1.5 & $<.02$ & .06 & -- \\
\hline $06 / 24 / 03$ & 905,000 & 364 & 25.4 & $<.01$ & 1.5 & $<.02$ & .07 & 5.6 \\
\hline $06 / 25 / 03$ & 905,000 & 366 & 26.1 & .01 & 1.5 & $<.02$ & .07 & 5.6 \\
\hline
\end{tabular}


Table 8. Results of analysis of streamflow, physical properties, and nutrients for water samples collected from three sampling sites in the lower Mississippi River, Louisiana, 1991-2003.-Continued

[Streamflow data from U.S. Army Corps of Engineers, New Orleans District. Physical property data from various collectors. Nutrient data from U.S. Geological Survey National Water-Quality Laboratory, Lakewood, Colorado; $\mathrm{ft}^{3} / \mathrm{s}$, cubic feet per second; $\mu \mathrm{S} / \mathrm{cm}$, microsiemens per centimeter at 25 degrees Celsius; ${ }^{\circ} \mathrm{C}$, degrees Celsius; $\mathrm{mg} / \mathrm{L}$, milligrams per liter; <, less than; --, no data]

\begin{tabular}{|c|c|c|c|c|c|c|c|c|}
\hline $\begin{array}{c}\text { Collection } \\
\text { (month/day/ } \\
\text { year) }\end{array}$ & $\begin{array}{c}\text { Streamflow } \\
\left(\mathrm{ft}^{3} / \mathrm{s}\right)\end{array}$ & $\begin{array}{c}\text { Specific } \\
\text { conductance } \\
(\mu \mathrm{S} / \mathrm{cm})\end{array}$ & $\begin{array}{c}\text { Water } \\
\text { temperature } \\
\left({ }^{\circ} \mathrm{C}\right)\end{array}$ & $\begin{array}{c}\text { Nitrite as } \\
\text { nitrogen } \\
\text { (mg/L) }\end{array}$ & $\begin{array}{l}\text { Nitrite plus } \\
\text { nitrate as } \\
\text { nitrogen } \\
\text { (mg/L) }\end{array}$ & $\begin{array}{l}\text { Ammonia } \\
\text { as nitrogen } \\
(\mathrm{mg} / \mathrm{L})\end{array}$ & $\begin{array}{l}\text { Orthophosphate } \\
\text { as phosphorus } \\
\text { (mg/L) }\end{array}$ & $\begin{array}{c}\text { Silica as } \\
\mathrm{SiO}_{2} \text { (mg/L) }\end{array}$ \\
\hline \multicolumn{9}{|c|}{ Mississippi River at Baton Rouge, Louisiana, station 07374000 (fig. 1)—Continued } \\
\hline $06 / 26 / 03$ & 898,000 & 348 & 26.0 & 0.01 & 1.5 & $<0.02$ & 0.06 & -- \\
\hline $06 / 27 / 03$ & 875,000 & 333 & 26.0 & .01 & 1.5 & $<.02$ & .06 & 5.5 \\
\hline 06/30/03 & 839,000 & -- & -- & .01 & 1.5 & $<.02$ & .07 & -- \\
\hline 07/01/03 & 823,000 & 354 & 25.6 & .01 & 1.6 & $<.02$ & .07 & 6.0 \\
\hline 07/02/03 & 791,000 & 369 & 27.0 & $<.01$ & 1.6 & $<.02$ & .07 & -- \\
\hline 07/03/03 & 764,000 & 359 & 26.8 & $<.01$ & 1.7 & $<.02$ & .07 & 6.1 \\
\hline 07/07/03 & 621,000 & 386 & 27.2 & $<.01$ & 1.4 & $<.02$ & .07 & -- \\
\hline 07/08/03 & 569,000 & 365 & 28.2 & .01 & 1.4 & $<.02$ & .06 & 6.2 \\
\hline $07 / 15 / 03$ & 459,000 & 400 & 26.8 & $<.01$ & 1.3 & $<.02$ & .07 & 5.3 \\
\hline $07 / 30 / 03$ & 566,000 & 407 & 26.9 & $<.01$ & 2.0 & $<.02$ & .04 & 7.4 \\
\hline 09/09/03 & 348,000 & 420 & -- & -- & .45 & -- & -- & -- \\
\hline 09/10/03 & 390,000 & 415 & 28.7 & -- & .41 & -- & -- & -- \\
\hline $09 / 11 / 03$ & 447,000 & 412 & 28.5 & -- & .42 & -- & -- & -- \\
\hline $09 / 15 / 03$ & 630,000 & 397 & 27.8 & -- & .84 & -- & -- & -- \\
\hline 09/17/03 & 640,000 & -- & -- & -- & .91 & -- & -- & -- \\
\hline 09/18/03 & 603,000 & 374 & 26.6 & -- & .90 & -- & -- & -- \\
\hline $09 / 23 / 03$ & -- & 366 & 26.3 & -- & .89 & -- & -- & -- \\
\hline $09 / 29 / 03$ & 330,000 & 428 & 25.1 & $<.01$ & .60 & $<.02$ & .07 & 6.2 \\
\hline $10 / 15 / 03$ & 367,000 & 408 & 22.0 & $<.01$ & .75 & .11 & .06 & 6.2 \\
\hline $11 / 05 / 03$ & 289,000 & 431 & 21.7 & $<.01$ & .68 & .03 & .05 & 5.3 \\
\hline $12 / 10 / 03$ & 778,000 & 337 & 10.2 & $<.01$ & 1.2 & .02 & .05 & 6.5 \\
\hline \multicolumn{9}{|c|}{ Mississippi River at New Orleans, Louisiana (fig. 1) } \\
\hline $10 / 07 / 92$ & 506,000 & -- & -- & -- & 1.2 & -- & -- & -- \\
\hline $10 / 14 / 92$ & 335,000 & -- & -- & -- & 1.3 & -- & -- & -- \\
\hline $10 / 21 / 92$ & 271,000 & -- & -- & -- & 1.5 & -- & -- & -- \\
\hline $11 / 04 / 92$ & 303,000 & -- & -- & -- & 1.2 & -- & -- & -- \\
\hline $11 / 12 / 92$ & 310,000 & -- & -- & -- & 1.2 & -- & -- & -- \\
\hline $11 / 18 / 92$ & 471,000 & -- & -- & -- & 1.3 & -- & -- & -- \\
\hline $11 / 25 / 92$ & 675,000 & -- & -- & -- & 1.3 & -- & -- & -- \\
\hline $12 / 02 / 92$ & 895,000 & -- & -- & -- & 1.6 & -- & -- & -- \\
\hline $12 / 09 / 92$ & $1,045,000$ & -- & -- & -- & 2.2 & -- & -- & -- \\
\hline $12 / 16 / 92$ & 764,000 & -- & -- & -- & 1.8 & -- & -- & -- \\
\hline $01 / 06 / 93$ & 992,000 & -- & -- & -- & 1.7 & -- & -- & -- \\
\hline
\end{tabular}


Table 8. Results of analysis of streamflow, physical properties, and nutrients for water samples collected from three sampling sites in the lower Mississippi River, Louisiana, 1991-2003.-Continued

[Streamflow data from U.S. Army Corps of Engineers, New Orleans District. Physical property data from various collectors. Nutrient data from U.S. Geological Survey National Water-Quality Laboratory, Lakewood, Colorado; $\mathrm{ft}^{3} / \mathrm{s}$, cubic feet per second; $\mu \mathrm{S} / \mathrm{cm}$, microsiemens per centimeter at 25 degrees Celsius; ${ }^{\circ} \mathrm{C}$, degrees Celsius; $\mathrm{mg} / \mathrm{L}$, milligrams per liter; $<$, less than; --, no data]

\begin{tabular}{|c|c|c|c|c|c|c|c|c|}
\hline $\begin{array}{c}\text { Collection } \\
\text { (month/day/ } \\
\text { year) }\end{array}$ & $\begin{array}{c}\text { Streamflow } \\
\left(\mathrm{ft}^{3} / \mathrm{s}\right)\end{array}$ & $\begin{array}{c}\text { Specific } \\
\text { conductance } \\
(\mu \mathrm{S} / \mathrm{cm})\end{array}$ & $\begin{array}{c}\text { Water } \\
\text { temperature } \\
\left({ }^{\circ} \mathrm{C}\right)\end{array}$ & $\begin{array}{l}\text { Nitrite as } \\
\text { nitrogen } \\
\text { (mg/L) }\end{array}$ & $\begin{array}{l}\text { Nitrite plus } \\
\text { nitrate as } \\
\text { nitrogen } \\
\text { (mg/L) }\end{array}$ & $\begin{array}{l}\text { Ammonia } \\
\text { as nitrogen } \\
\text { (mg/L) }\end{array}$ & $\begin{array}{c}\text { Orthophosphate } \\
\text { as phosphorus } \\
\text { (mg/L) }\end{array}$ & $\begin{array}{c}\text { Silica as } \\
\mathrm{SiO}_{2}(\mathrm{mg} / \mathrm{L})\end{array}$ \\
\hline \multicolumn{9}{|c|}{ Mississippi River at New Orleans, Louisiana (fig. 1)—Continued } \\
\hline $01 / 13 / 93$ & $1,064,000$ & -- & -- & -- & 1.9 & -- & -- & -- \\
\hline $01 / 20 / 93$ & $1,135,000$ & -- & -- & -- & 1.9 & -- & -- & -- \\
\hline $01 / 27 / 93$ & $1,100,000$ & -- & -- & -- & 1.5 & -- & -- & -- \\
\hline $02 / 03 / 93$ & $1,075,000$ & -- & -- & -- & 1.9 & -- & -- & -- \\
\hline $02 / 10 / 93$ & 963,000 & -- & -- & -- & 1.8 & -- & -- & -- \\
\hline $02 / 17 / 93$ & 720,000 & -- & -- & -- & 1.8 & -- & -- & -- \\
\hline $02 / 24 / 93$ & 733,000 & -- & -- & -- & 1.7 & -- & -- & -- \\
\hline 03/03/93 & 894,000 & -- & -- & -- & 1.8 & -- & -- & -- \\
\hline 03/10/93 & 976,000 & -- & -- & -- & 1.6 & -- & -- & -- \\
\hline $03 / 17 / 93$ & $1,118,000$ & -- & -- & -- & 1.4 & -- & -- & -- \\
\hline $03 / 24 / 93$ & $1,256,000$ & -- & -- & -- & 1.7 & -- & -- & -- \\
\hline $03 / 31 / 93$ & $1,194,000$ & -- & -- & -- & 2.0 & -- & -- & -- \\
\hline 04/07/93 & $1,275,000$ & -- & -- & -- & 1.9 & -- & -- & -- \\
\hline $04 / 14 / 93$ & $1,421,000$ & -- & -- & -- & 1.6 & -- & -- & -- \\
\hline $04 / 21 / 93$ & $1,491,000$ & -- & -- & -- & 1.6 & -- & -- & -- \\
\hline $04 / 28 / 93$ & $1,525,000$ & -- & -- & -- & 1.6 & -- & -- & -- \\
\hline $05 / 05 / 93$ & $1,474,000$ & -- & -- & -- & 1.8 & -- & -- & -- \\
\hline $05 / 12 / 93$ & $1,452,000$ & -- & -- & -- & 2.0 & -- & -- & -- \\
\hline 05/19/93 & $1,549,000$ & -- & -- & -- & 1.9 & -- & -- & -- \\
\hline $05 / 26 / 93$ & $1,465,000$ & -- & -- & -- & 1.8 & -- & -- & -- \\
\hline $06 / 02 / 93$ & $1,276,000$ & -- & -- & -- & 1.8 & -- & -- & -- \\
\hline 06/09/93 & 980,000 & -- & -- & -- & 1.8 & -- & -- & -- \\
\hline $06 / 16 / 93$ & 869,000 & -- & -- & -- & 1.7 & -- & -- & -- \\
\hline $06 / 23 / 93$ & 916,000 & -- & -- & -- & 2.0 & -- & -- & -- \\
\hline $06 / 30 / 93$ & 840,000 & -- & -- & -- & 2.1 & -- & -- & -- \\
\hline 07/07/93 & 843,000 & -- & -- & -- & 2.5 & -- & -- & -- \\
\hline $07 / 14 / 93$ & 890,000 & -- & -- & -- & 2.1 & -- & -- & -- \\
\hline $07 / 21 / 93$ & 982,000 & -- & -- & -- & 2.7 & -- & -- & -- \\
\hline $07 / 28 / 93$ & $1,044,000$ & -- & -- & -- & 2.3 & -- & -- & -- \\
\hline 08/04/93 & $1,102,000$ & -- & -- & -- & 2.0 & -- & -- & -- \\
\hline 08/11/93 & $1,150,000$ & -- & -- & -- & 2.0 & -- & -- & -- \\
\hline 08/18/93 & $1,163,000$ & -- & -- & -- & 1.7 & -- & -- & -- \\
\hline $08 / 25 / 93$ & $1,113,000$ & -- & -- & -- & 1.7 & -- & -- & -- \\
\hline
\end{tabular}


Table 8. Results of analysis of streamflow, physical properties, and nutrients for water samples collected from three sampling sites in the lower Mississippi River, Louisiana, 1991-2003.-Continued

[Streamflow data from U.S. Army Corps of Engineers, New Orleans District. Physical property data from various collectors. Nutrient data from U.S. Geological Survey National Water-Quality Laboratory, Lakewood, Colorado; $\mathrm{ft}^{3} / \mathrm{s}$, cubic feet per second; $\mu \mathrm{S} / \mathrm{cm}$, microsiemens per centimeter at 25 degrees Celsius; ${ }^{\circ} \mathrm{C}$, degrees Celsius; mg/L, milligrams per liter; <, less than; --, no data]

\begin{tabular}{|c|c|c|c|c|c|c|c|c|}
\hline $\begin{array}{l}\text { Collection } \\
\text { (month/day/ } \\
\text { year) }\end{array}$ & $\begin{array}{l}\text { Streamflow } \\
\left(\mathrm{ft}^{3} / \mathrm{s}\right)\end{array}$ & $\begin{array}{c}\text { Specific } \\
\text { conductance } \\
(\mu \mathrm{S} / \mathrm{cm})\end{array}$ & $\begin{array}{c}\text { Water } \\
\text { temperature } \\
\left({ }^{\circ} \mathrm{C}\right)\end{array}$ & $\begin{array}{l}\text { Nitrite as } \\
\text { nitrogen } \\
\text { (mg/L) }\end{array}$ & $\begin{array}{c}\text { Nitrite plus } \\
\text { nitrate as } \\
\text { nitrogen } \\
\text { (mg/L) }\end{array}$ & $\begin{array}{l}\text { Ammonia } \\
\text { as nitrogen } \\
(\mathrm{mg} / \mathrm{L})\end{array}$ & $\begin{array}{l}\text { Orthophosphate } \\
\text { as phosphorus } \\
(\mathrm{mg} / \mathrm{L})\end{array}$ & $\begin{array}{c}\text { Silica as } \\
\mathrm{SiO}_{2} \text { (mg/L) }\end{array}$ \\
\hline \multicolumn{9}{|c|}{ Mississippi River at New Orleans, Louisiana (fig. 1)—Continued } \\
\hline 09/01/93 & $1,020,000$ & -- & -- & -- & 1.5 & -- & -- & -- \\
\hline 09/08/93 & 754,000 & -- & -- & -- & 1.3 & -- & -- & -- \\
\hline 09/15/93 & 682,000 & -- & -- & -- & 1.4 & -- & -- & -- \\
\hline 09/22/93 & 680,000 & -- & -- & -- & 1.6 & -- & -- & -- \\
\hline 09/29/93 & 721,000 & -- & -- & -- & 1.9 & -- & -- & -- \\
\hline $10 / 06 / 93$ & 928,000 & -- & -- & -- & 1.6 & -- & -- & -- \\
\hline $10 / 13 / 93$ & $1,008,000$ & -- & -- & -- & 1.3 & -- & -- & -- \\
\hline $10 / 20 / 93$ & 738,000 & -- & -- & -- & 1.3 & -- & -- & -- \\
\hline $10 / 27 / 93$ & 570,000 & -- & -- & -- & 1.4 & -- & -- & -- \\
\hline $11 / 03 / 93$ & 561,000 & -- & -- & -- & 1.4 & -- & -- & -- \\
\hline
\end{tabular}


Table 9. Statistical summary of concentrations of selected herbicides and degradation products analyzed by the U.S. Geological Survey National Water-Quality Laboratory, Lakewood, Colorado, for water samples collected from the Mississippi River near St. Francisville and at Baton Rouge, Louisiana, 1991-2003.

[MRL, method reporting limit; <, less than]

\begin{tabular}{|c|c|c|c|c|c|c|c|}
\hline \multirow{2}{*}{ Herbicide } & \multirow{2}{*}{$\begin{array}{c}\text { Number of } \\
\text { samples }\end{array}$} & \multirow{2}{*}{$\begin{array}{c}\text { Number of } \\
\text { samples at or } \\
\text { above MRL }\end{array}$} & \multicolumn{5}{|c|}{ Concentration, in micrograms per liter } \\
\hline & & & Mean & Median & 75th percentile & 95th percentile & Maximum \\
\hline \multicolumn{8}{|c|}{ Mississippi River near Saint Francisville, Louisiana, station 007373420 (fig. 1) } \\
\hline Acetochlor & 118 & 101 & 0.077 & 0.02 & 0.12 & 0.36 & 0.56 \\
\hline Alachlor & 144 & 77 & .020 & .01 & .02 & .11 & .22 \\
\hline Atrazine & 144 & 144 & 689 & .26 & .91 & 2.3 & 4.7 \\
\hline Cyanazine & 142 & 85 & .096 & .01 & .06 & .74 & 1.2 \\
\hline Deethylatrazine & 142 & 132 & .069 & .04 & .10 & .22 & .45 \\
\hline Deisopropylatrazine & 31 & 2 & .006 & $<.05$ & $<.05$ & .08 & .10 \\
\hline Metolachlor & 144 & 136 & .261 & .09 & .35 & .99 & 2.4 \\
\hline Metribuzin & 144 & 47 & .008 & $<.05$ & .01 & .04 & .12 \\
\hline Prometon & 142 & 81 & .007 & .01 & .01 & .01 & .14 \\
\hline Prometryn & 31 & 0 & $<.05$ & $<.05$ & $<.05$ & $<.05$ & $<.05$ \\
\hline Propachlor & 118 & 1 & .001 & $<.05$ & $<.05$ & $<.05$ & .13 \\
\hline Simazine & 142 & 126 & .070 & .05 & .09 & .25 & .41 \\
\hline \multicolumn{8}{|c|}{ Mississippi River at Baton Rouge, Louisiana, station 07374000 (fig. 1) } \\
\hline Alachlor & 84 & 40 & .069 & $<.05$ & .10 & .30 & .46 \\
\hline Atrazine & 84 & 78 & .668 & .365 & 1.1 & 2.5 & 3.6 \\
\hline Cyanazine & 84 & 38 & .245 & $<.20$ & .30 & 1.1 & 1.8 \\
\hline Deethylatrazine & 84 & 61 & .099 & .070 & .16 & .32 & .39 \\
\hline Deisopropylatrazine & 84 & 15 & .025 & $<.05$ & $<.05$ & .16 & .22 \\
\hline Metolachlor & 84 & 72 & .249 & .150 & .37 & .87 & 1.4 \\
\hline Metribuzin & 84 & 18 & .020 & $<.05$ & $<.05$ & .10 & .20 \\
\hline Prometon & 84 & 12 & .011 & $<.05$ & $<.05$ & .08 & .16 \\
\hline Prometryn & 84 & 2 & .002 & $<.05$ & $<.05$ & $<.05$ & .07 \\
\hline Simazine & 84 & 35 & .034 & $<.05$ & .08 & .10 & .14 \\
\hline
\end{tabular}


Table 10. Statistical summary of concentrations of selected herbicides analyzed by the Jefferson Parish Water Quality Laboratory, Jefferson, Louisiana, for water samples collected from the Mississippi River at New Orleans, Louisiana, March through September 1993.

[MRL, method reporting limit; <, less than]

\begin{tabular}{lccccccc}
\hline Herbicide & \multirow{2}{*}{$\begin{array}{c}\text { Number of } \\
\text { samples }\end{array}$} & $\begin{array}{c}\text { Number of } \\
\text { samples at or } \\
\text { above MRL }\end{array}$ & Mean & Median & 75th percentile 95th percentile & Maximum \\
\hline Alachlor & 26 & 19 & 0.116 & 0.040 & 0.12 & 0.36 & 0.40 \\
Atrazine & 26 & 26 & .088 & .680 & 1.3 & 3.8 & 4.2 \\
Metolachlor & 26 & 26 & .335 & .175 & .39 & 1.3 & .31 \\
Simazine & 26 & 25 & .120 & .100 & .18 & .35 \\
\hline
\end{tabular}


Table 11. Statistical summary of concentrations of selected triazine herbicides and degradation products analyzed by gas chromatography/mass spectrometry methods GCS (1993-96) and GCR (1995-2003) at the U.S. Geological Survey Organic Geochemistry Research Laboratory, Lawrence, Kansas, for water samples collected from the Mississippi River at Baton Rouge, Louisiana, 1993-2003.

[MRL, method reporting limit; ESA, ethanesulfonic acid; <, less than]

\begin{tabular}{|c|c|c|c|c|c|c|c|}
\hline \multirow{2}{*}{ Herbicides } & \multirow{2}{*}{$\begin{array}{c}\text { Number of } \\
\text { samples }\end{array}$} & \multirow{2}{*}{$\begin{array}{l}\text { Number of } \\
\text { samples at or } \\
\text { above MRL }\end{array}$} & \multicolumn{5}{|c|}{ Concentration, in micrograms per liter } \\
\hline & & & Mean & Median & 75th percentile & 95th percentile & Maximum \\
\hline Acetochlor & 228 & 50 & 0.036 & $<0.05$ & $<0.05$ & 0.20 & 0.49 \\
\hline Alachlor & 275 & 40 & .018 & $<.05$ & $<.05$ & .13 & .37 \\
\hline Alachlor ESA & 169 & 165 & .710 & .52 & .86 & 2.0 & 3.7 \\
\hline Atrazine & 275 & 272 & .610 & .27 & .86 & 2.1 & 3.3 \\
\hline Deethylatrazine & 275 & 197 & .124 & .07 & .17 & .43 & .52 \\
\hline Deisopropylatrazine & 275 & 122 & .070 & $<.05$ & .10 & .31 & .53 \\
\hline Cyanazine & 275 & 100 & .159 & $<.05$ & .14 & .89 & 1.5 \\
\hline Cyanazine amide & 214 & 65 & .129 & $<.05$ & .10 & .79 & 1.2 \\
\hline Demethyl-fluometuron & 178 & 3 & .003 & $<.05$ & $<.05$ & $<.05$ & .19 \\
\hline Dimethenamid & 91 & 13 & .015 & $<.05$ & $<.05$ & .11 & .23 \\
\hline Fluometuron & 175 & 13 & .007 & $<.05$ & $<.05$ & .08 & .17 \\
\hline Metolachlor & 275 & 196 & .219 & .10 & .39 & .80 & 1.1 \\
\hline Metribuzin & 275 & 10 & .003 & $<.05$ & $<.05$ & $<.05$ & .13 \\
\hline Molinate & 175 & 4 & .002 & $<.05$ & $<.05$ & $<.05$ & .10 \\
\hline Prometon & 129 & 4 & .002 & $<.05$ & $<.05$ & $<.05$ & .07 \\
\hline Simazine & 275 & 87 & .040 & $<.05$ & .06 & .20 & .95 \\
\hline Trifluralin & 173 & 1 & .0003 & $<.05$ & $<.05$ & $<.05$ & .06 \\
\hline 3-trifluomethyl-phenylurea & 175 & 1 & .0003 & $<.05$ & $<.05$ & $<.05$ & .06 \\
\hline
\end{tabular}


Table 12. Statistical summary of concentrations of selected acetamide herbicides and degradation products analyzed by liquid chromatography/mass spectrometry methods HPAA (1998-2001), LCAA (1999-2002), and LCPD (2002-03) at the U.S. Geological Survey Organic Geochemistry Research Laboratory, Lawrence, Kansas, for water samples collected from the Mississippi River at Baton Rouge, Louisiana, 1998-2003.

[MRL, method reporting limit; ESA, ethanesulfonic acid; OXA, oxanilic acid; <, less than]

\begin{tabular}{|c|c|c|c|c|c|c|c|}
\hline \multirow{2}{*}{ Herbicide } & \multirow{2}{*}{$\begin{array}{c}\text { Number of } \\
\text { samples }\end{array}$} & \multirow{2}{*}{$\begin{array}{l}\text { Number of } \\
\text { samples at or } \\
\text { above MRL }\end{array}$} & \multicolumn{5}{|c|}{ Concentration, in micrograms per liter } \\
\hline & & & Mean & Median & 75th percentile & 95th percentile & Maximum \\
\hline Acetochlor & 18 & 5 & 0.024 & $<0.025$ & 0.05 & 0.14 & 0.14 \\
\hline Acetochlor ESA & 126 & 81 & .115 & .07 & .17 & .40 & .58 \\
\hline Acetochlor OXA & 126 & 79 & .113 & .06 & .17 & .42 & .60 \\
\hline Acetochlor SA & 36 & 11 & .033 & $<.025$ & .03 & .19 & .27 \\
\hline Alachlor & 18 & 0 & $<.05$ & $<.05$ & $<.05$ & $<.05$ & $<.05$ \\
\hline Alachlor ESA & 126 & 100 & .105 & .09 & .15 & .27 & .44 \\
\hline Alachlor OXA & 126 & 27 & .011 & $<.05$ & $<.05$ & .07 & .12 \\
\hline Alachlor SA & 36 & 2 & .002 & $<.05$ & $<.05$ & .02 & .04 \\
\hline Alachlor ESA 2nd amide & 18 & 1 & .002 & $<.05$ & $<.05$ & .03 & .03 \\
\hline Dimethenamid & 18 & 3 & .007 & $<.05$ & $<.05$ & .06 & .06 \\
\hline Dimethenamid ESA & 91 & 15 & .008 & $<.05$ & $<.05$ & .06 & .11 \\
\hline Dimethenamid OXA & 91 & 10 & .006 & $<.05$ & $<.05$ & .05 & .11 \\
\hline Flufenacet & 18 & 0 & $<.05$ & $<.05$ & $<.05$ & $<.05$ & $<.05$ \\
\hline Flufenacet SA & 18 & 0 & $<.05$ & $<.05$ & $<.05$ & $<.05$ & $<.05$ \\
\hline Flufenacet $\mathrm{OA}$ & 18 & 0 & $<.05$ & $<.05$ & $<.05$ & $<.05$ & $<.05$ \\
\hline Metolachlor & 18 & 14 & .091 & .03 & .16 & .38 & .38 \\
\hline Metolachlor ESA & 126 & 122 & .372 & .295 & .51 & .84 & 1.3 \\
\hline Metolachlor OXA & 126 & 100 & .147 & .11 & .22 & .45 & .57 \\
\hline Metolachlor ESA 2nd amide & 18 & 9 & .021 & .01 & .04 & .09 & .09 \\
\hline Propachlor & 18 & 0 & $<.05$ & $<.05$ & $<.05$ & $<.05$ & $<.05$ \\
\hline Propachlor ESA & 36 & 1 & .002 & $<.05$ & $<.05$ & $<.05$ & .06 \\
\hline Propachlor OA & 18 & 0 & $<.05$ & $<.05$ & $<.05$ & $<.05$ & $<.05$ \\
\hline
\end{tabular}


Table 13. Statistical summary of concentrations of selected triazine and phenylurea herbicides and degradation products analyzed by liquid chromatography/mass spectrometry method LCEA at the U.S. Geological Survey Organic Geochemistry Research Laboratory, Lawrence, Kansas, for water samples collected from the Mississippi River at Baton Rouge, Louisiana, 1998-2003.

[MRL, method reporting limit; <, less than]

\begin{tabular}{|c|c|c|c|c|c|c|c|}
\hline \multirow{2}{*}{ Herbicides } & \multirow{2}{*}{$\begin{array}{c}\text { Number of } \\
\text { samples }\end{array}$} & \multirow{2}{*}{$\begin{array}{c}\text { Number of } \\
\text { samples at or } \\
\text { above MRL }\end{array}$} & \multicolumn{5}{|c|}{ Concentration, in micrograms per liter } \\
\hline & & & Mean & Median & $\begin{array}{c}\text { 75th } \\
\text { percentile }\end{array}$ & $\begin{array}{c}\text { 95th } \\
\text { percentile }\end{array}$ & Maximum \\
\hline Atrazine & 33 & 33 & 0.351 & 0.27 & 0.45 & 1.1 & 1.3 \\
\hline Cyanazine & 33 & 1 & .002 & $<.025$ & $<.025$ & $<.025$ & .07 \\
\hline Cyanazine acid & 33 & 2 & .006 & $<.025$ & $<.025$ & .04 & .16 \\
\hline Deethylatrazine & 33 & 31 & .128 & .10 & .20 & .31 & .37 \\
\hline Deethylcyanazine acid & 33 & 2 & .022 & $<.025$ & $<.025$ & .37 & .37 \\
\hline Deisopropylatrazine & 33 & 21 & .064 & .05 & .11 & .17 & .21 \\
\hline Deisopropylhydroxyatrazine & 33 & 1 & .001 & $<.025$ & $<.025$ & $<.025$ & .04 \\
\hline Didealkylatrazine & 33 & 21 & .055 & .06 & .09 & .130 & .21 \\
\hline Diuron & 33 & 3 & .011 & $<.025$ & $<.025$ & .14 & .14 \\
\hline Hydroxyatrazine & 33 & 30 & .128 & .08 & .18 & .25 & .27 \\
\hline Simazine & 33 & 19 & .044 & .03 & .07 & .12 & .26 \\
\hline
\end{tabular}


Table 14. Statistical summary of concentrations of glyphosate, its degradation product aminomethylphosphonic acid, and glufosinate analyzed by liquid chromatography/mass spectrometry method LCGY at the U.S. Geological Survey Organic Geochemistry Research Laboratory, Lawrence, Kansas, for water samples collected from the Mississippi River at Baton Rouge, Louisiana, 2001-03.

[MRL, method reporting limit; AMPA, aminomethylphosphonic acid, <, less than]

\begin{tabular}{lccrrrrr}
\hline \multirow{2}{*}{ Constituent } & \multirow{2}{*}{$\begin{array}{c}\text { Number of } \\
\text { samples }\end{array}$} & $\begin{array}{c}\text { Number of } \\
\text { samples at or } \\
\text { above MRL }\end{array}$ & Mean & Median & 75th percentile & 95th percentile & Maximum \\
\cline { 5 - 9 } & 36 & 0 & $<0.10$ & $<0.10$ & $<0.10$ & $<0.10$ & $<0.10$ \\
Glyphosate & 36 & 32 & .18 & .18 & .24 & .33 & .38 \\
AMPA & 36 & 0 & $<.10$ & $<.10$ & $<.10$ & $<.10$ & $<.10$ \\
Glufosinate & & & & & & &
\end{tabular}


Table 15. Statistical summary of streamflow, physical properties, and nutrients analyzed for water samples collected from three sampling sites in the lower Mississippi River, Louisiana, 1991-2003.

[Streamflow data from U.S. Army Corps of Engineers, New Orleans District. Physical property data from various collectors. Nutrient data from U.S. Geological Survey National Water Quality Laboratory,Lakewood, Colorado. MRL, method reporting limit; $\mathrm{ft}^{3} / \mathrm{s}$, cubic feet per second; $\mu \mathrm{S} / \mathrm{cm}$, microsiemens per centimeter at 25 degrees Celsius; ${ }^{\circ} \mathrm{C}$, degrees Celsius; $\mathrm{mg} / \mathrm{L}$, milligrams per liter; <, less than]

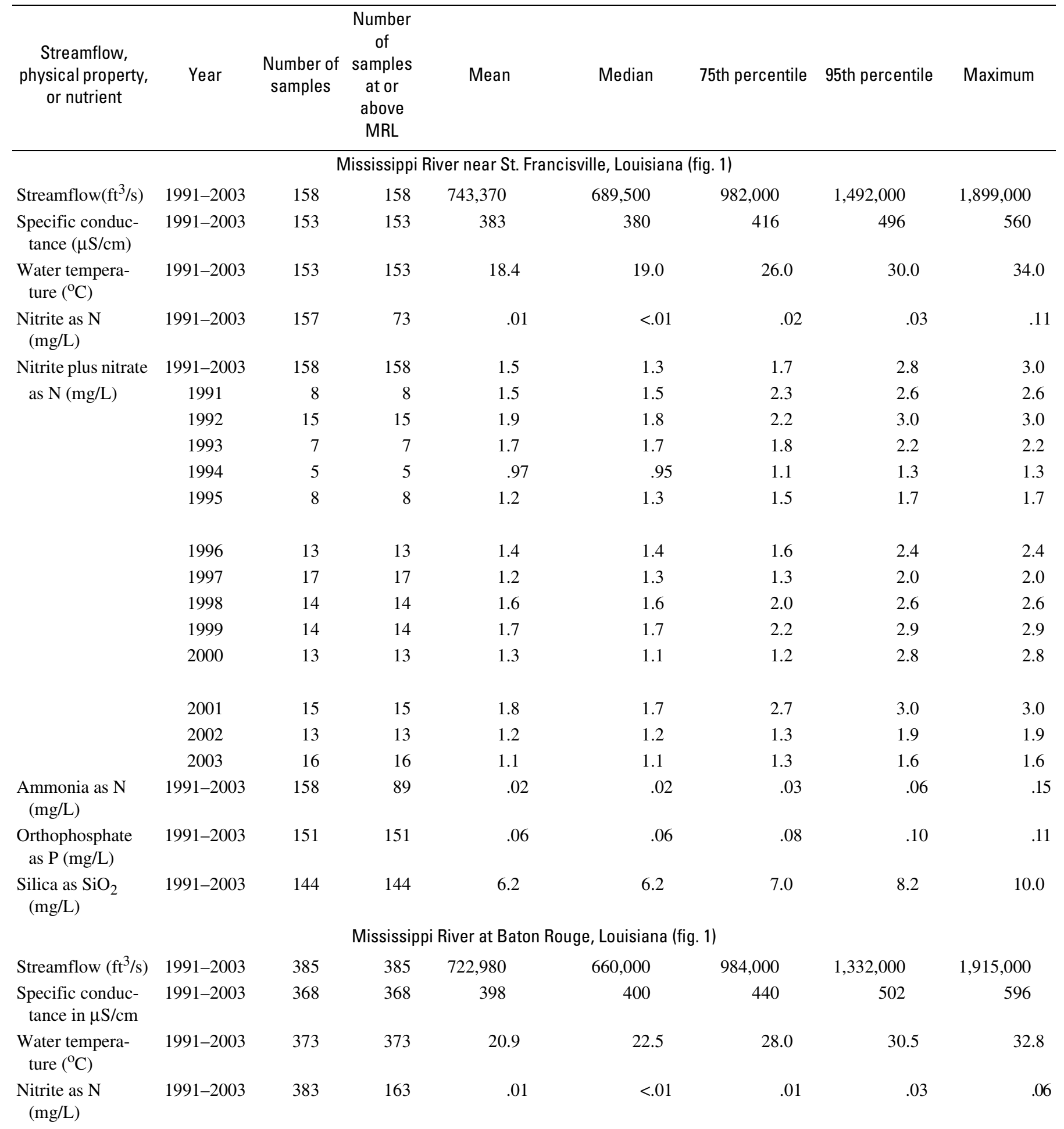


Table 15. Statistical summary of streamflow, physical properties, and nutrients analyzed for water samples collected from three sampling sites in the lower Mississippi River, Louisiana, 1991-2003.—Continued

[Streamflow data from U.S. Army Corps of Engineers, New Orleans District. Physical property data from various collectors. Nutrient data from U.S. Geological Survey National Water Quality Laboratory, Lakewood, Colorado. MRL, method reporting limit; $\mathrm{ft}^{3} / \mathrm{s}$, cubic feet per second; $\mu \mathrm{S} / \mathrm{cm}$, microsiemens per centimeter at 25 degrees Celsius; ${ }^{\circ} \mathrm{C}$, degrees Celsius; mg/L, milligrams per liter; <, less than]

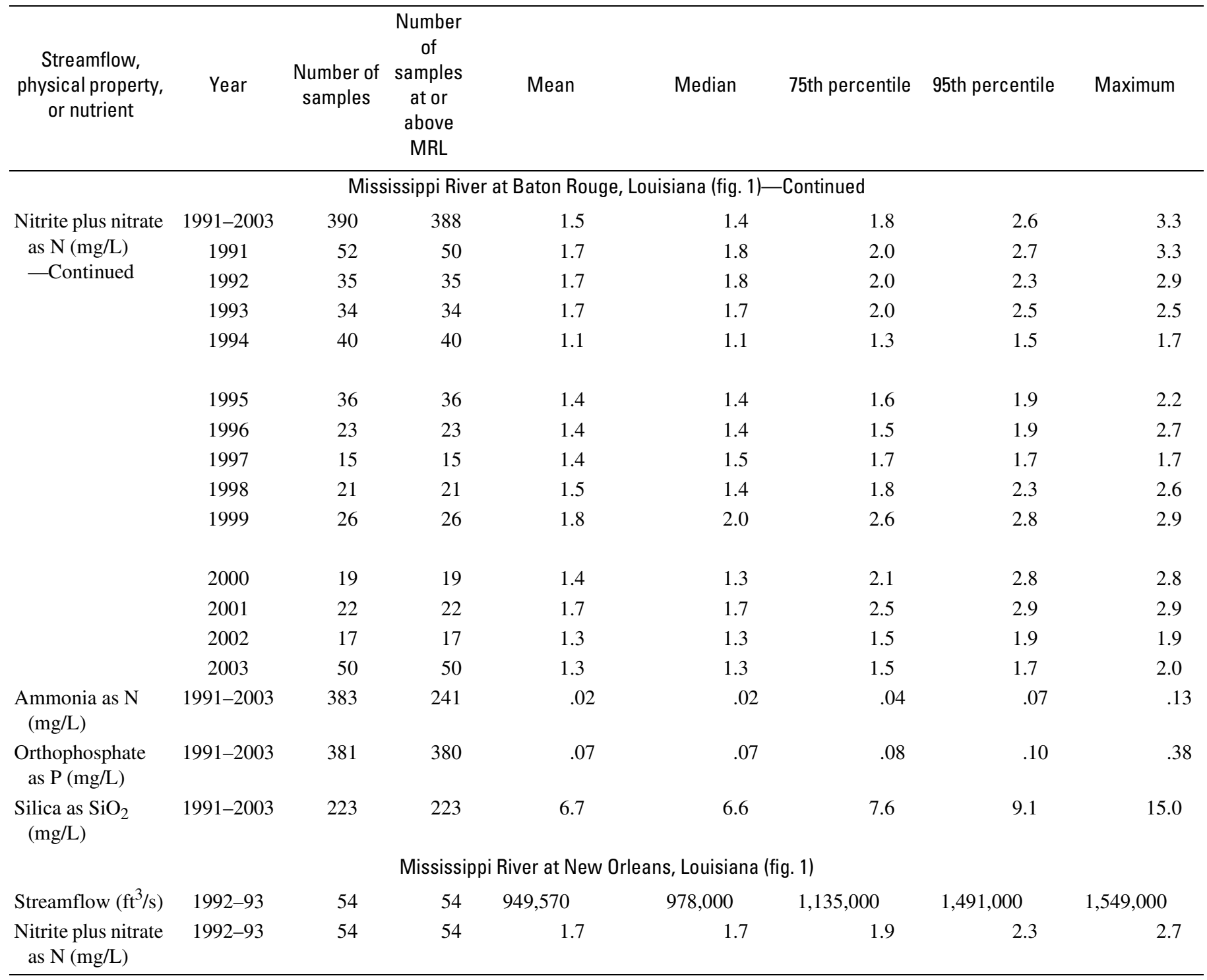

